\title{
Synthesis of 5-Membered Sulfur Heterocycles via Tin-Catalyzed Annulation of Mercapto Ketones with Activated Alkenes
}

Itaru Suzuki, ${ }^{1}$ Yuki, Sakamoto, ${ }^{1,2}$ Yuta Seo, Yoriko Ninomaru, Kodai Tokuda, and Ikuya Shibata*1

${ }^{1}$ Research Center for Environmental Preservation, Osaka University, 2-4 Yamadaoka, Suita, Osaka $565-$ 0871, Japan.

${ }^{2}$ Division of Applied Chemistry, Graduate School of Engineering, Osaka University, 2-1 Yamadaoka, Suita, Osaka 565-0871, Japan

e-mail: shibata@epc.osaka-u.ac.jp

Tel: +81-6-6879-8974

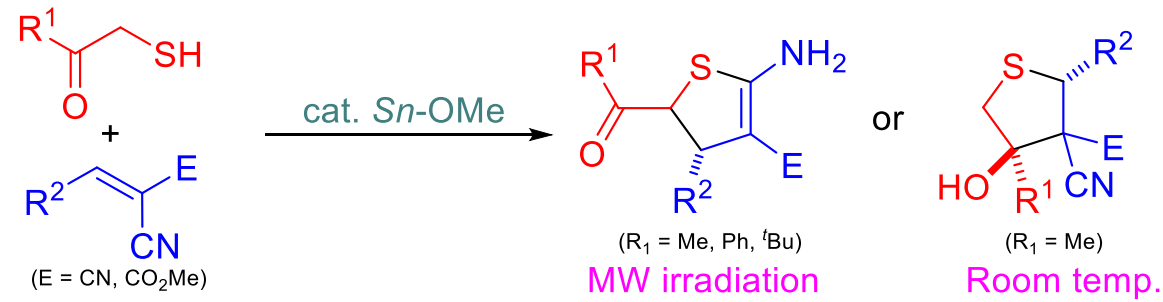




\section{Product Data}

5-Acetyl-2-amino-4-phenyl-4,5-dihydrothiophene-3-carbonitrile (trans-3aa)<smiles>CC(=O)C1SC(N)=C(C#N)[C@H]1c1ccccc1</smiles>

${ }^{1} \mathrm{H}$ NMR

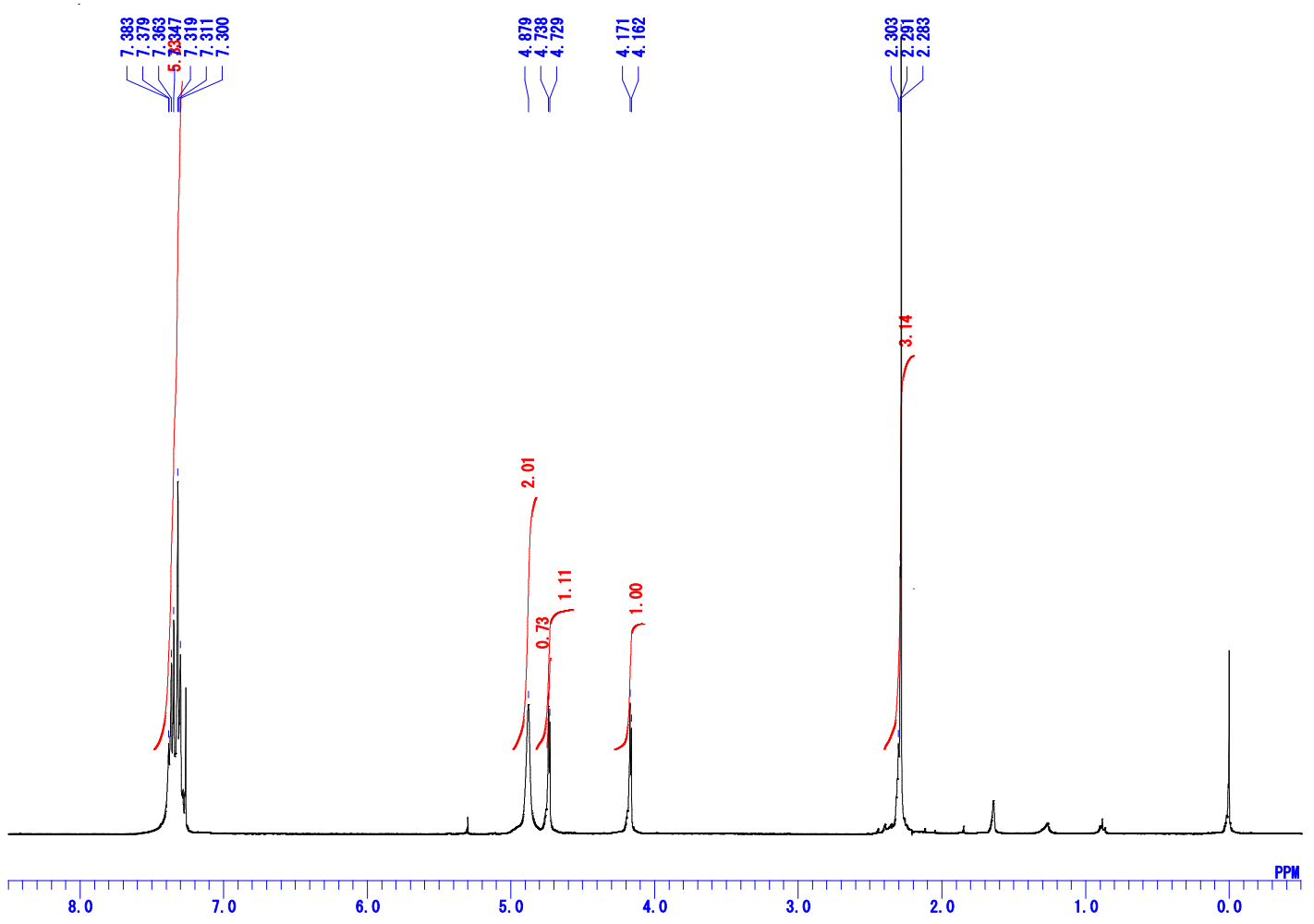


${ }^{13} \mathrm{C}$ NMR

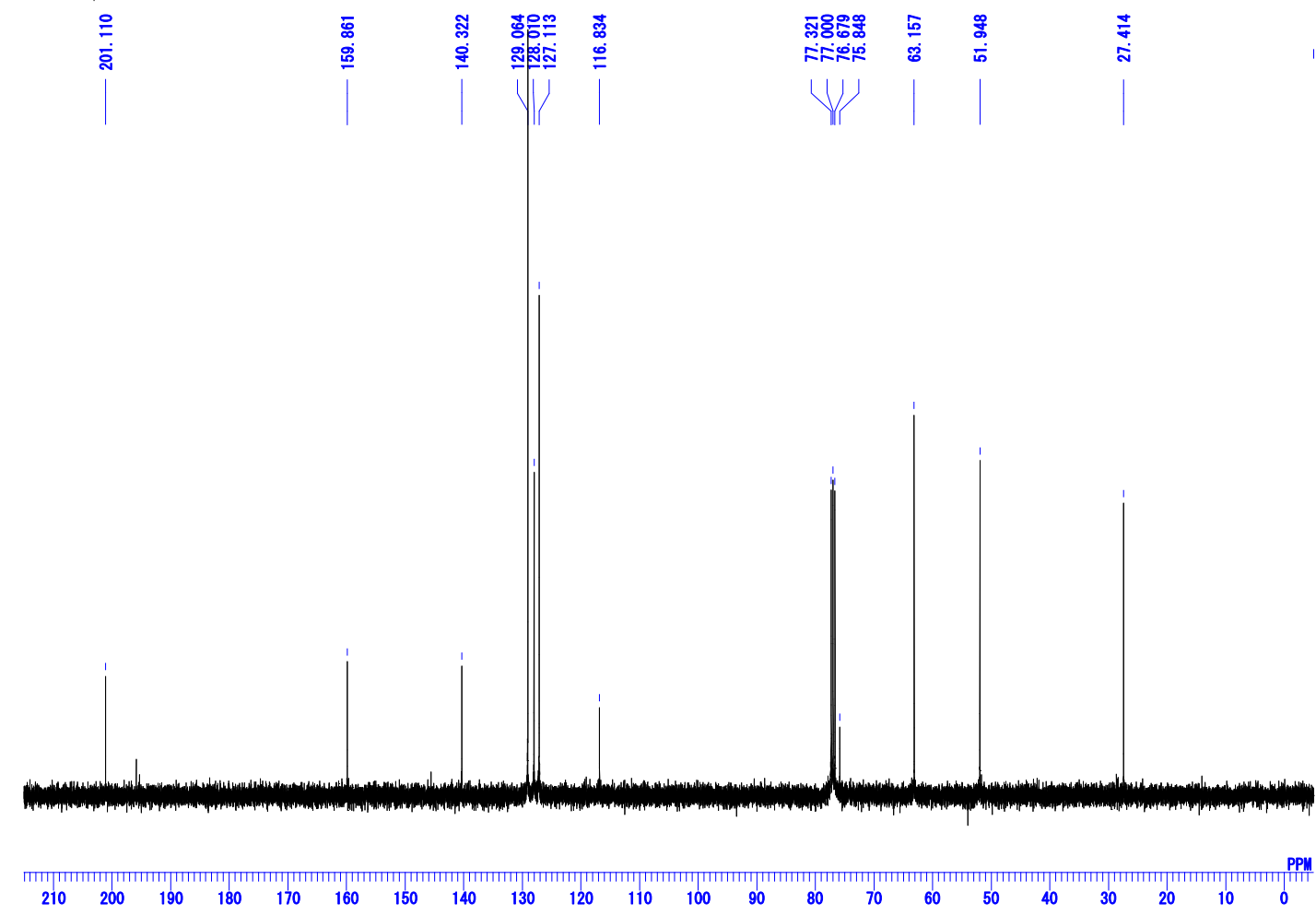

MW Profile

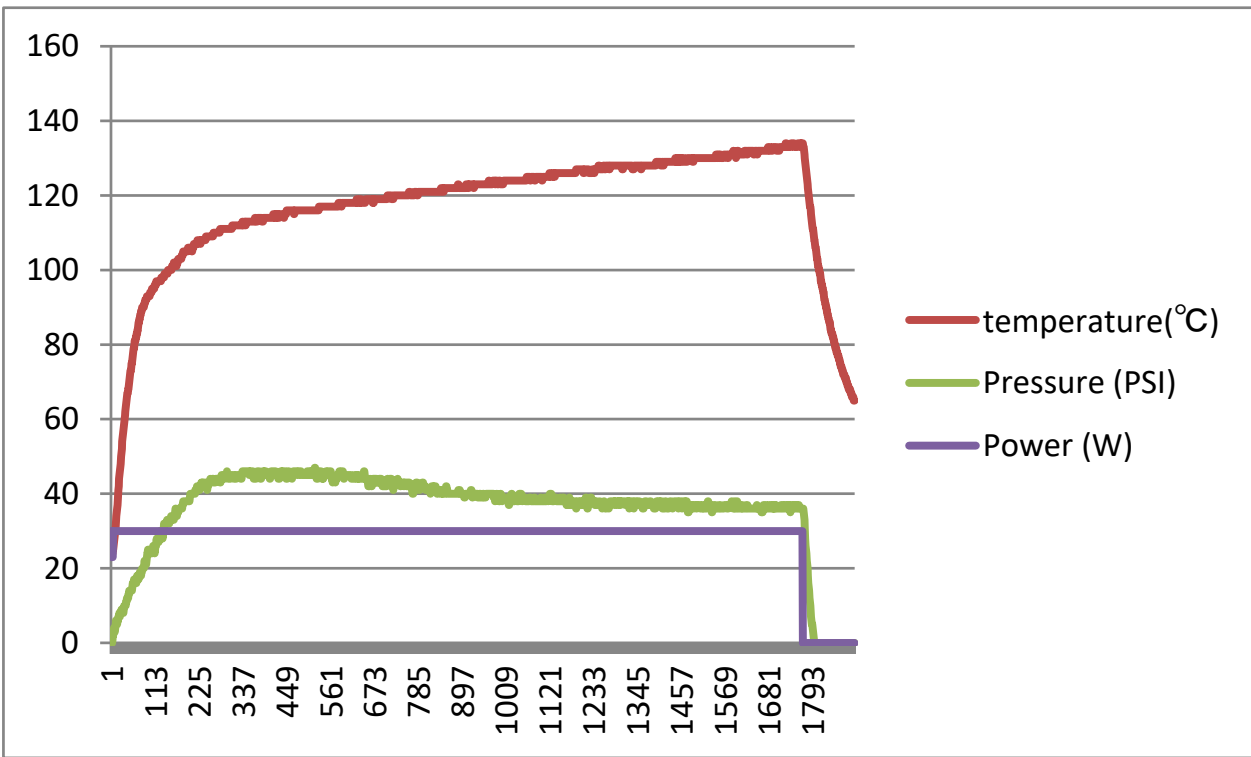


5-Acetyl-2-amino-4-phenyl-4,5-dihydrothiophene-3-carbonitrile (cis-3aa)<smiles>CC(=O)C1SC(N)=C(C#N)C1c1ccccc1</smiles>

${ }^{1} \mathrm{H}$ NMR

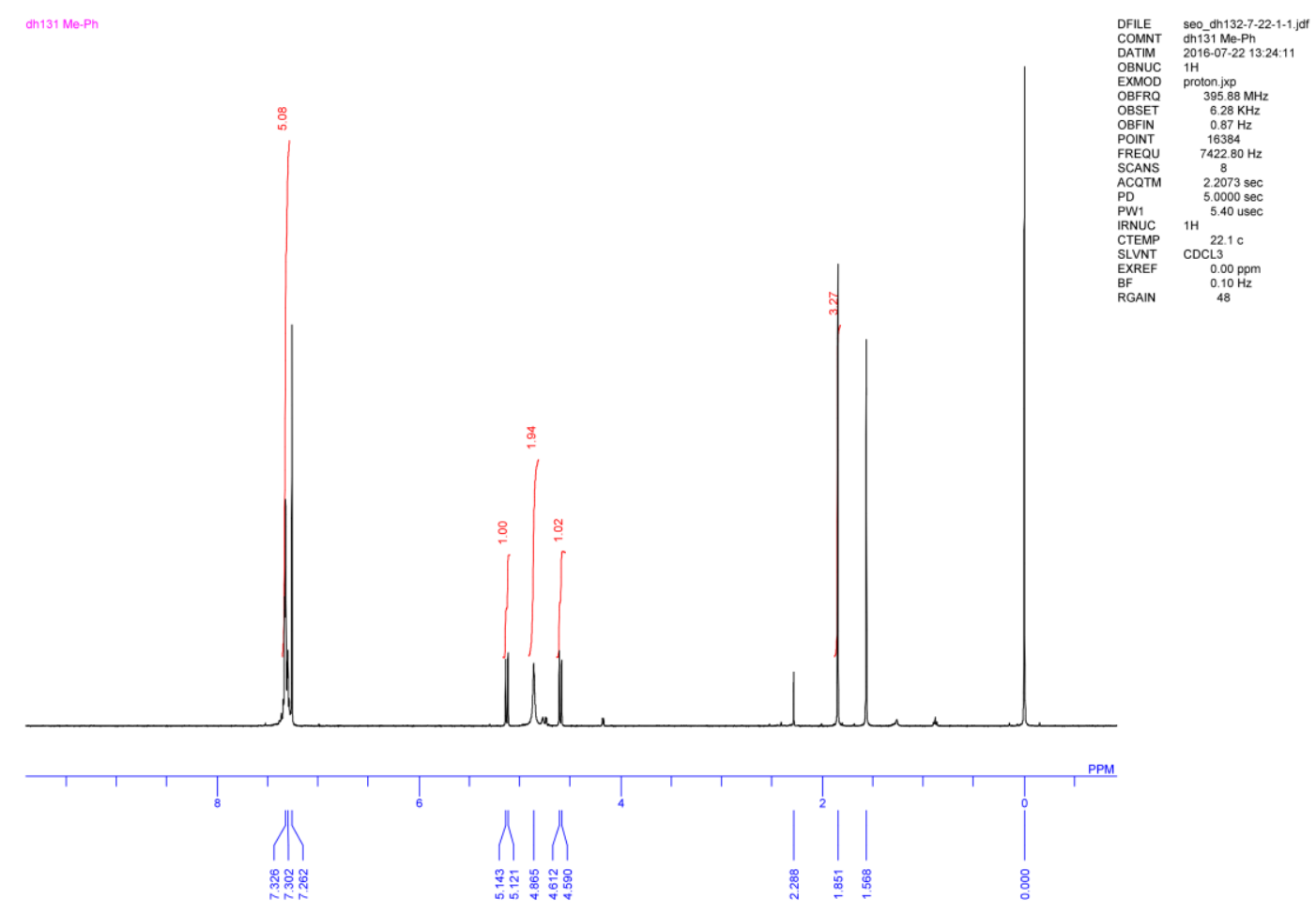


${ }^{13} \mathrm{C}$ NMR

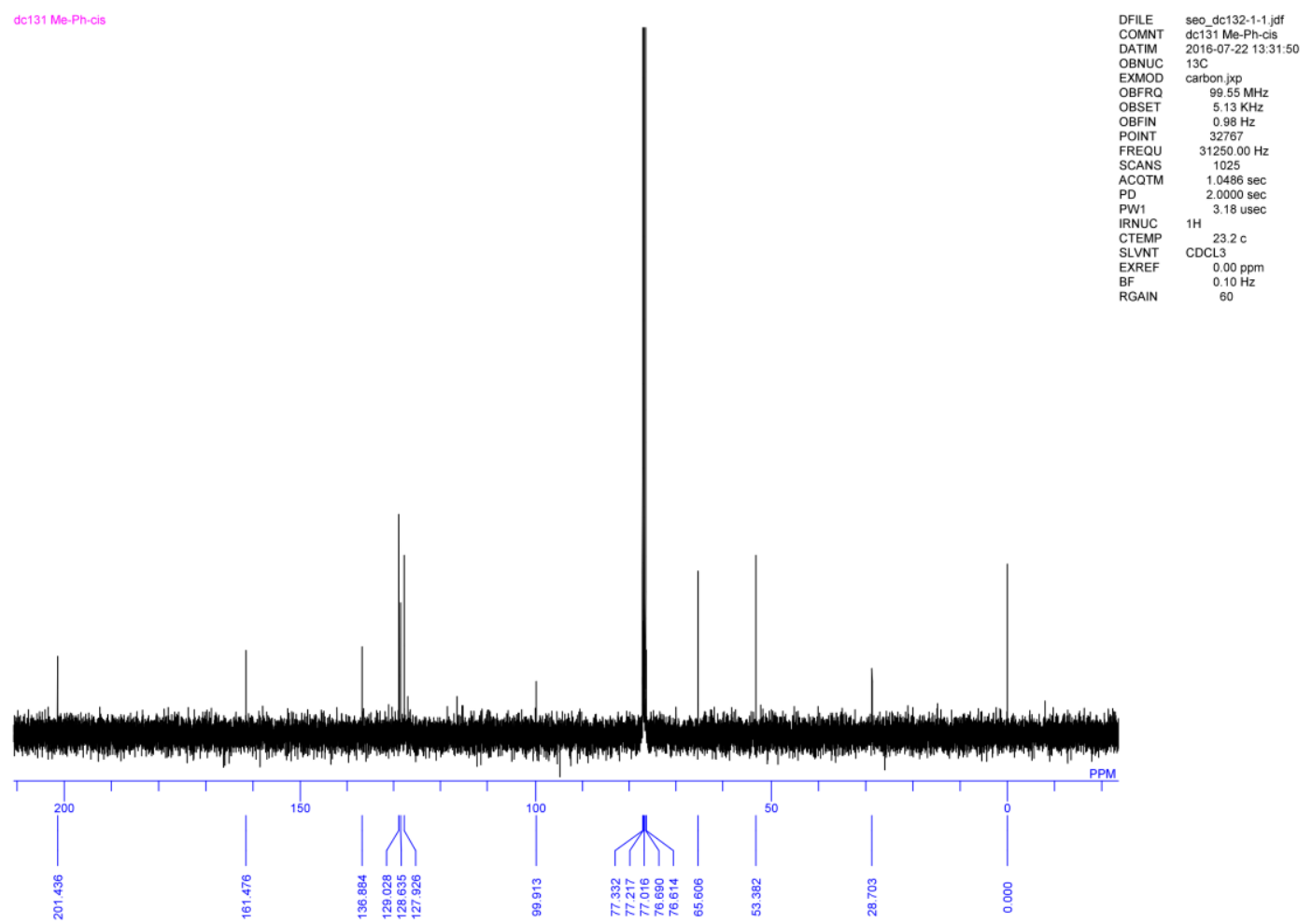


5-Acetyl-2-amino-4-(4-chlorophenyl)-4,5-dihydrothiophene-3-carbonitrile (trans-3ab)<smiles>CC(=O)C1SC(N)=C(C#N)[C@H]1c1ccc(Cl)cc1</smiles>

${ }^{1} \mathrm{H}$ NMNM

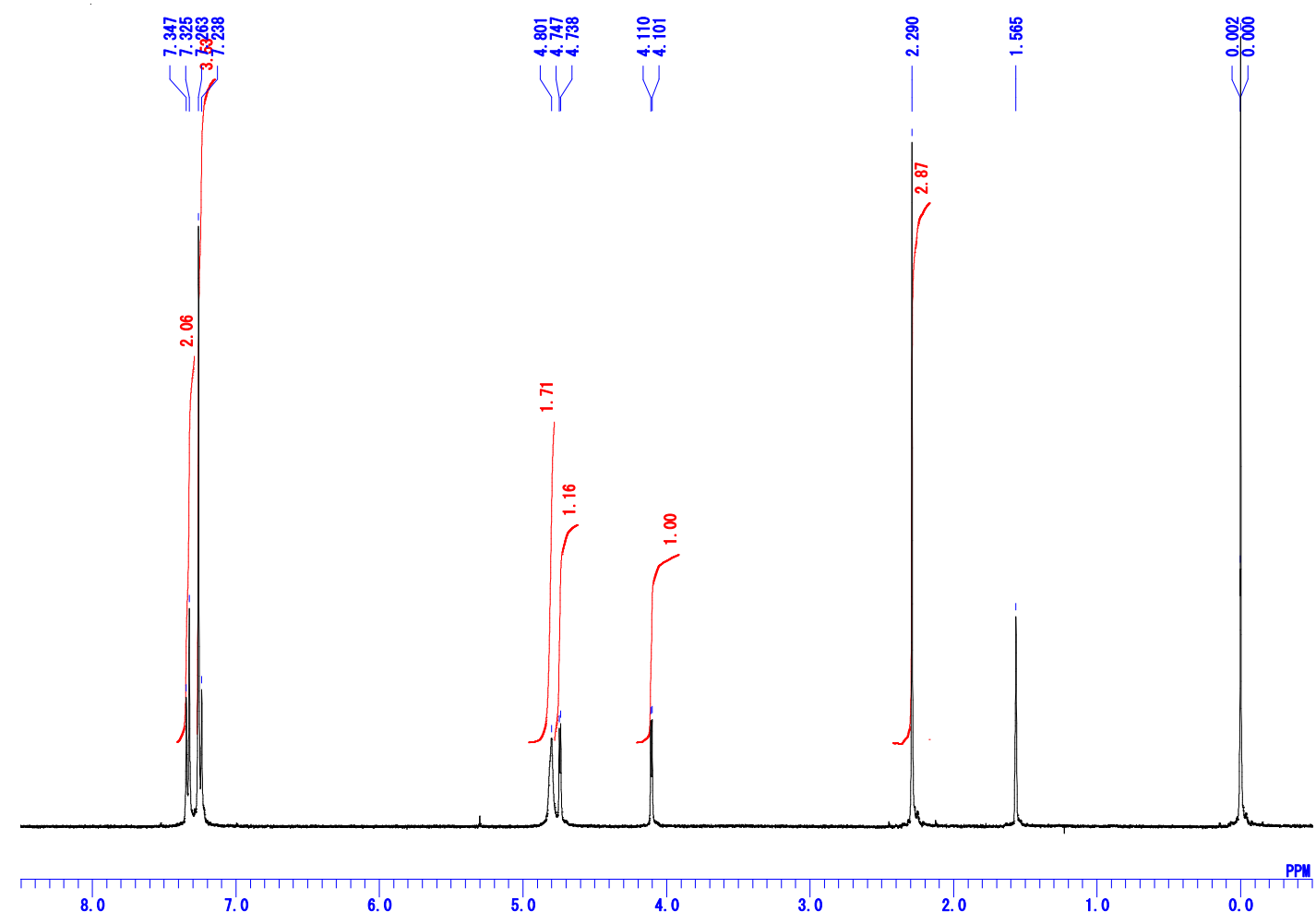


${ }^{13} \mathrm{C}$ NMR

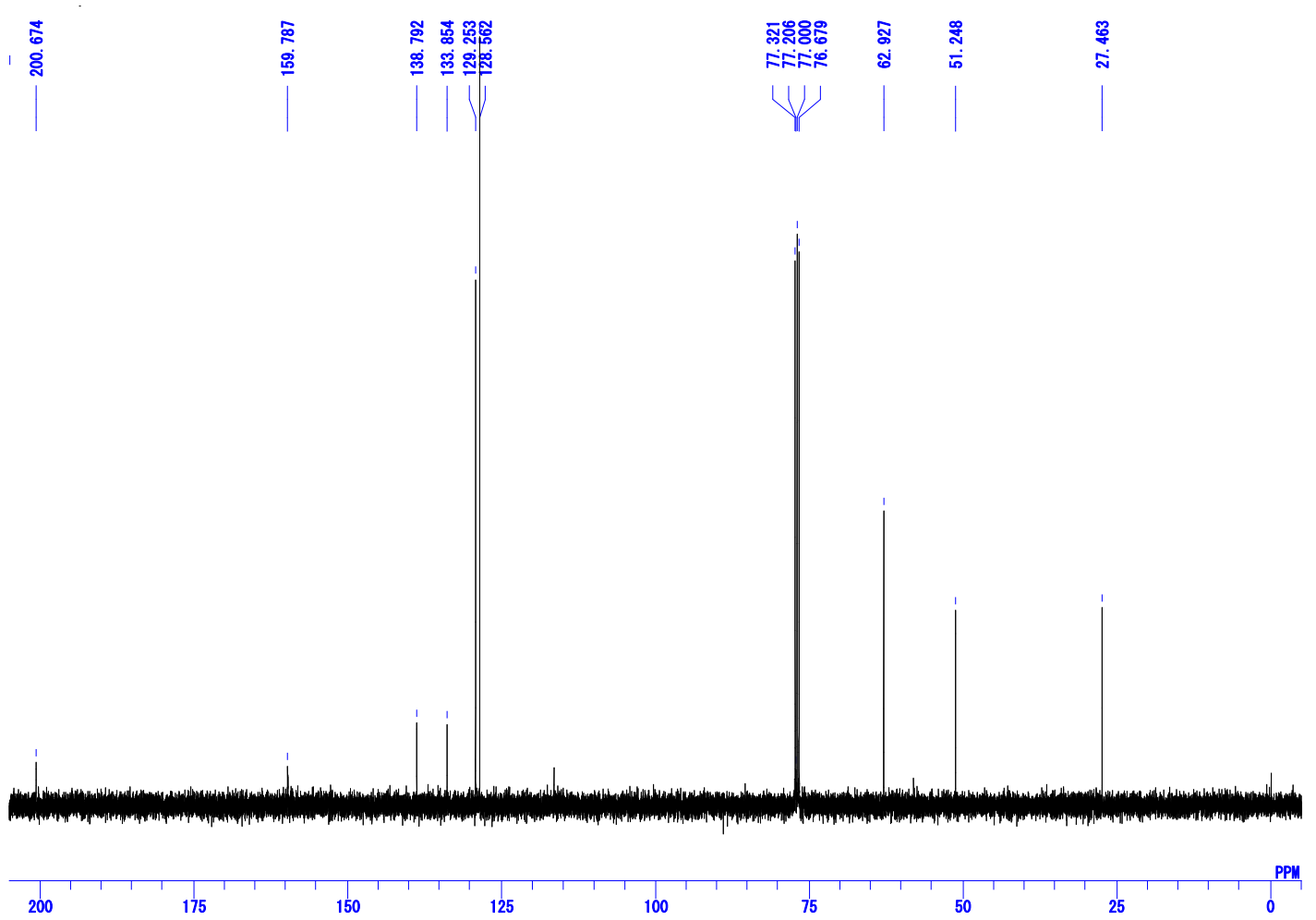

MW Profile

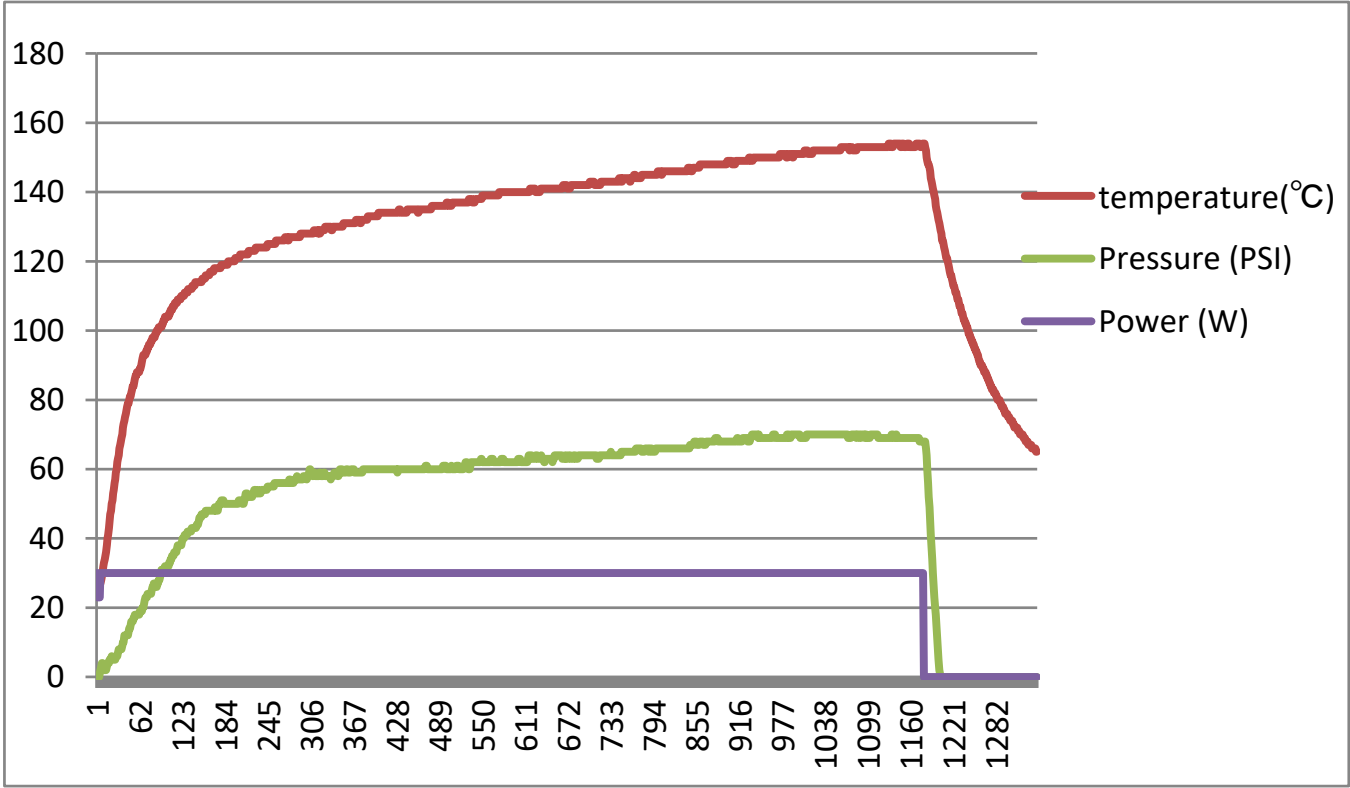


5-Acetyl-2-amino-5-methyl-4-(4-chlorophenyl)-4,5-dihydrofuran-3-carbonitrile (cis-3ab)<smiles>CC(=O)C1SC(N)=C(C#N)C1c1ccc(Cl)cc1</smiles>

${ }^{1} \mathrm{H}$ NMR

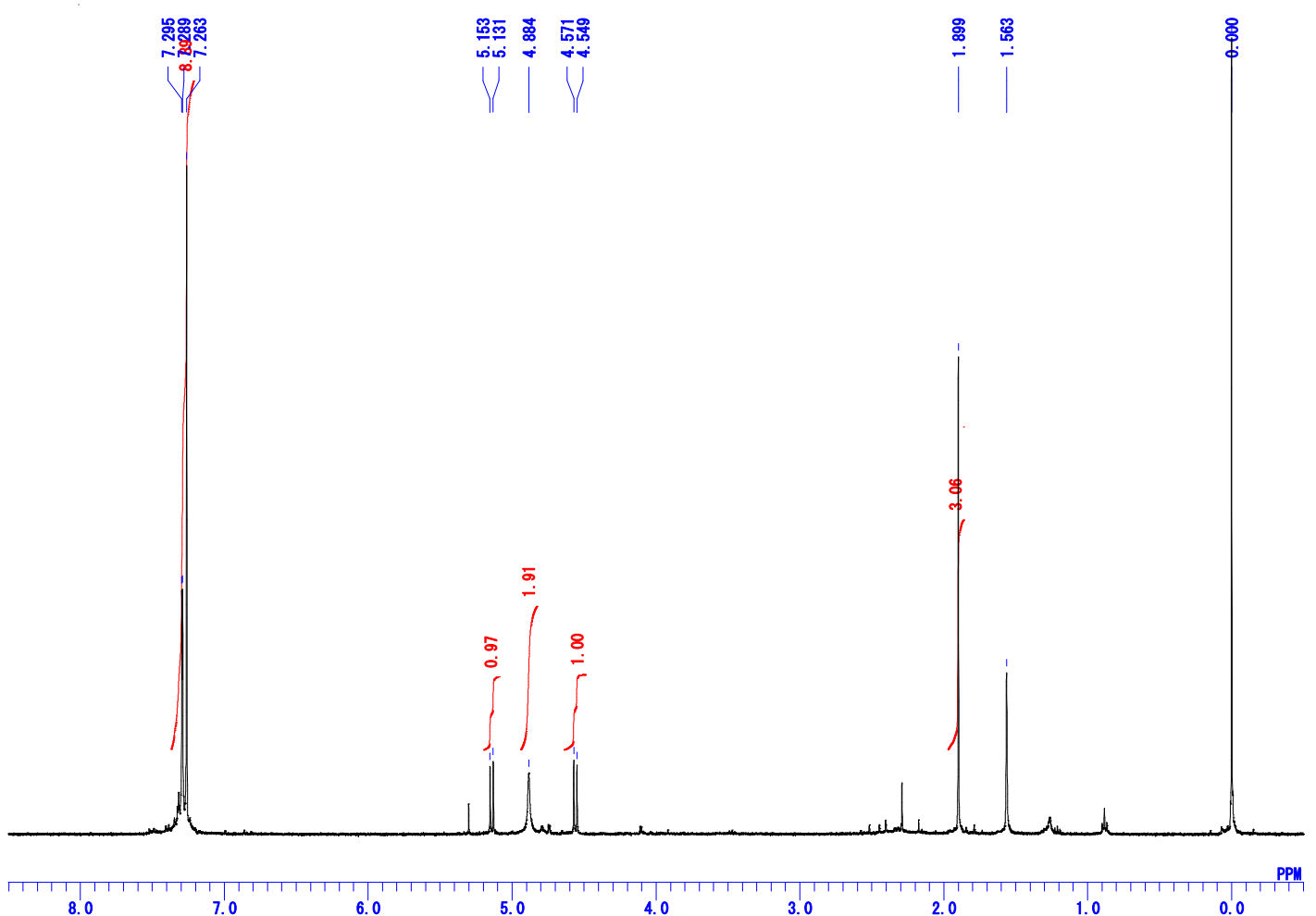


${ }^{13} \mathrm{C}$ NMR

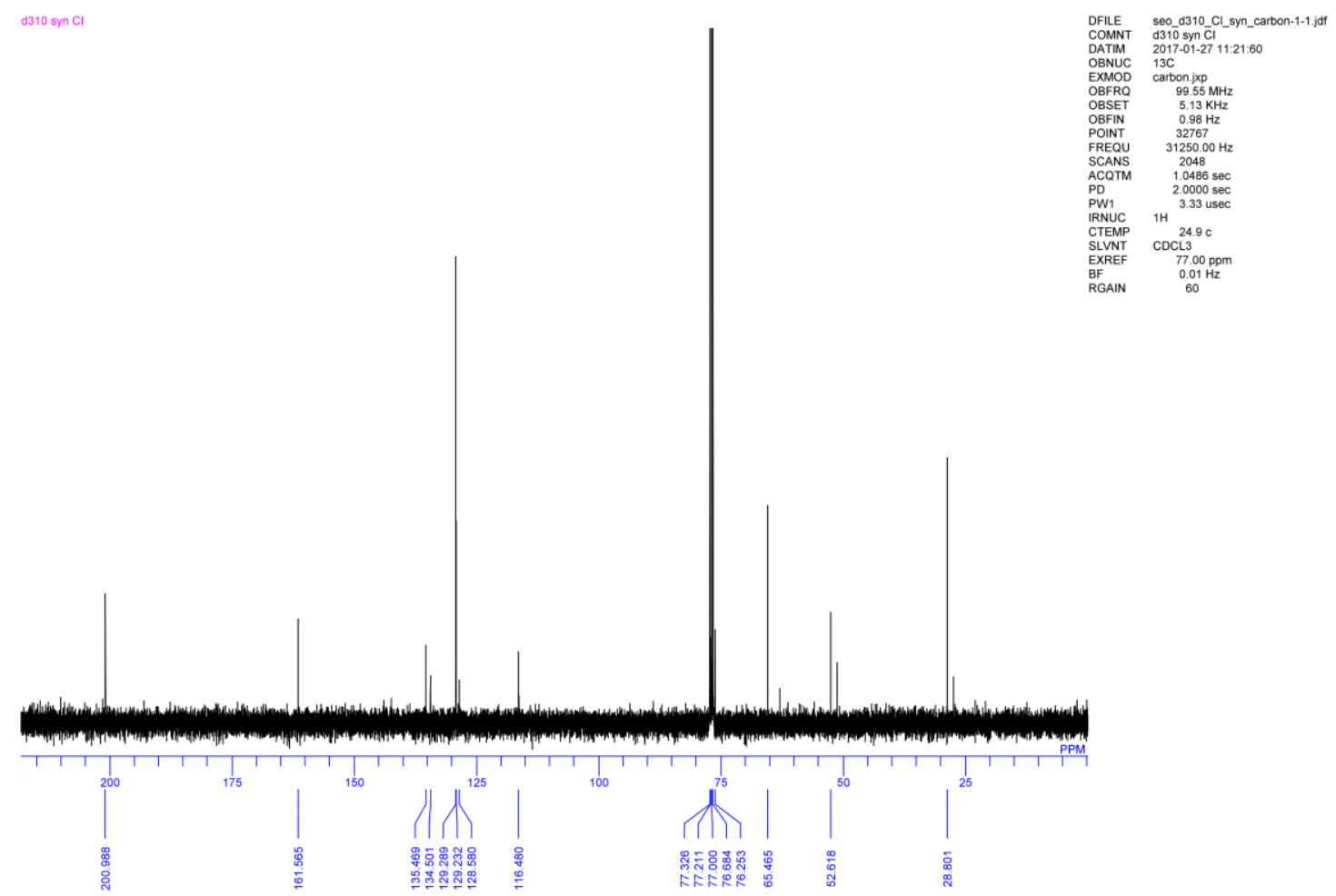


5-Acetyl-2-amino-4-( $p$-tolyl)-4,5-dihydrothiophene-3-carbonitrile (trans-3ac)<smiles>CC(=O)C1SC(N)=C(C#N)[C@H]1c1ccc(C)cc1</smiles>

\section{${ }^{1} \mathrm{H}$ NMR}

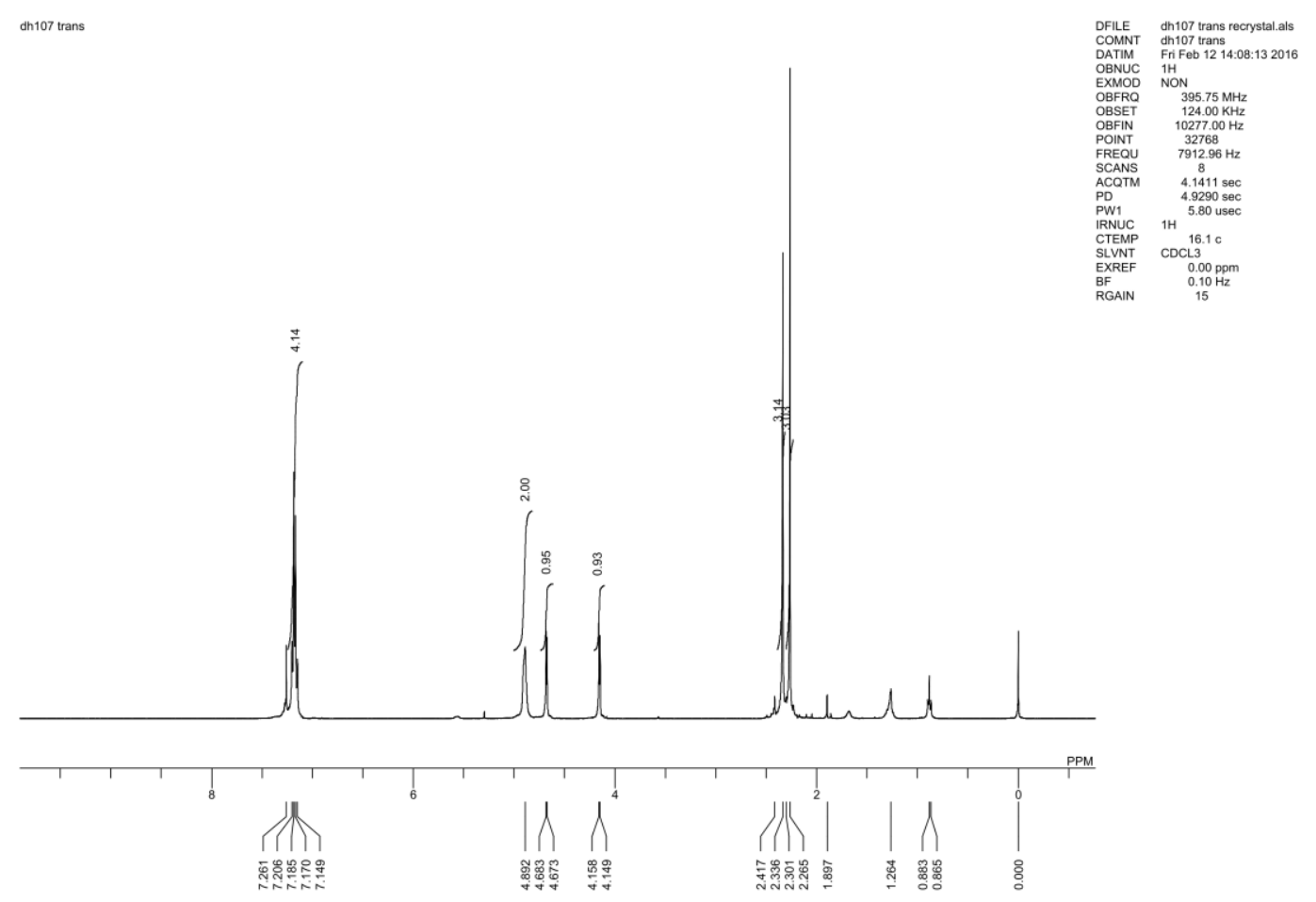


${ }^{13} \mathrm{C}$ NMR

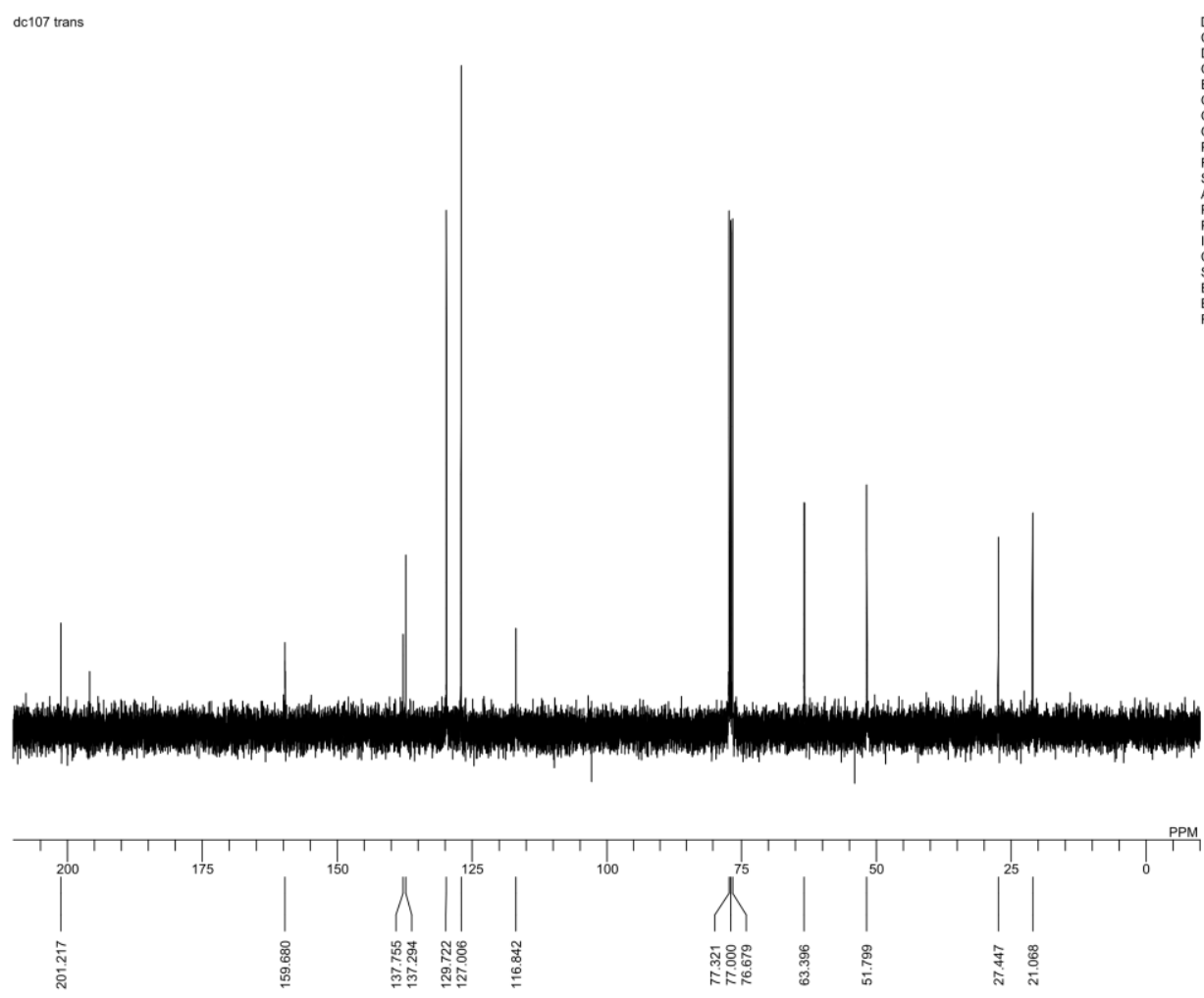

MW profile

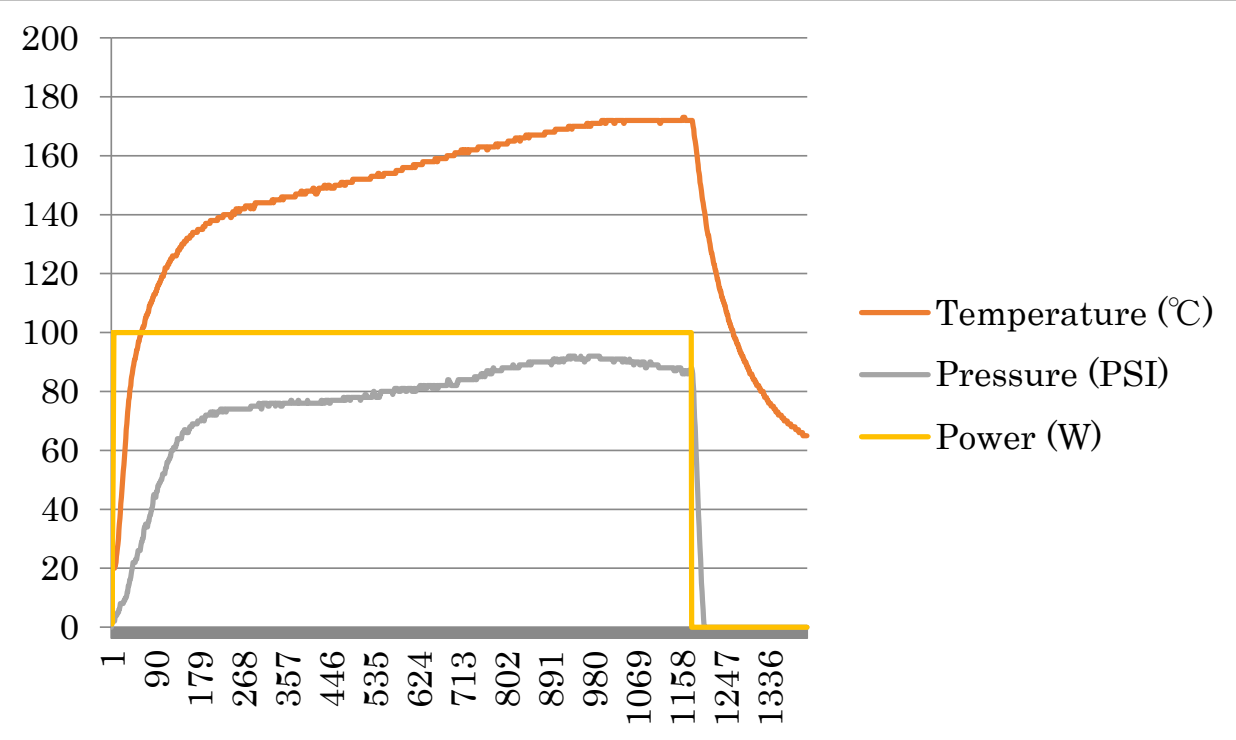


5-Acetyl-2-amino-4-(p-tolyl)-4,5-dihydrothiophene-3-carbonitrile (cis-3ac)

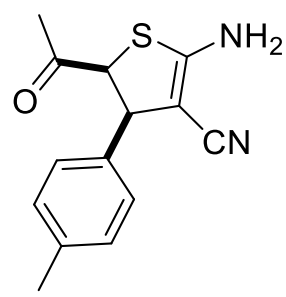

${ }^{1} \mathrm{H}$ NMR

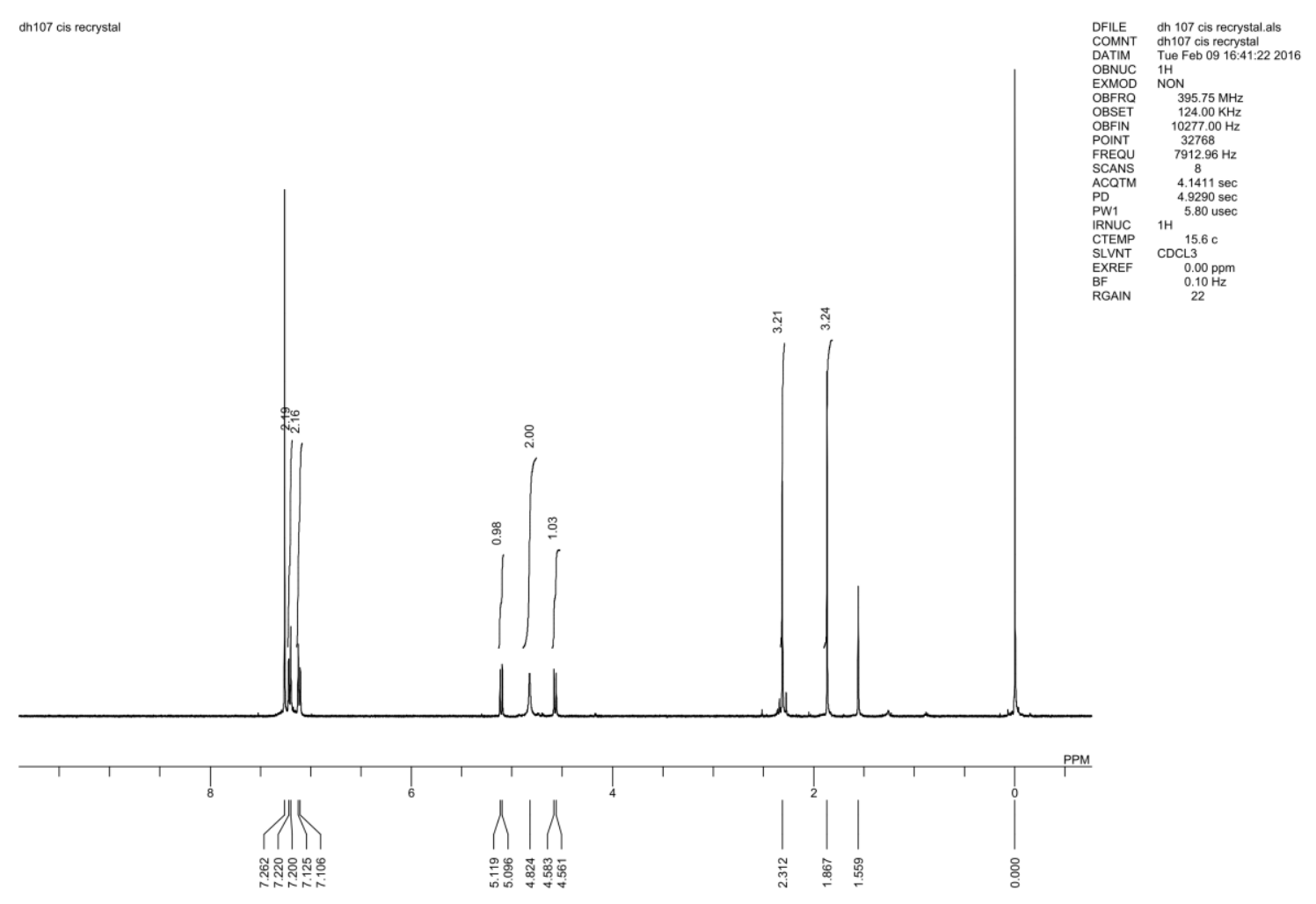


${ }^{13} \mathrm{C}$ NMR

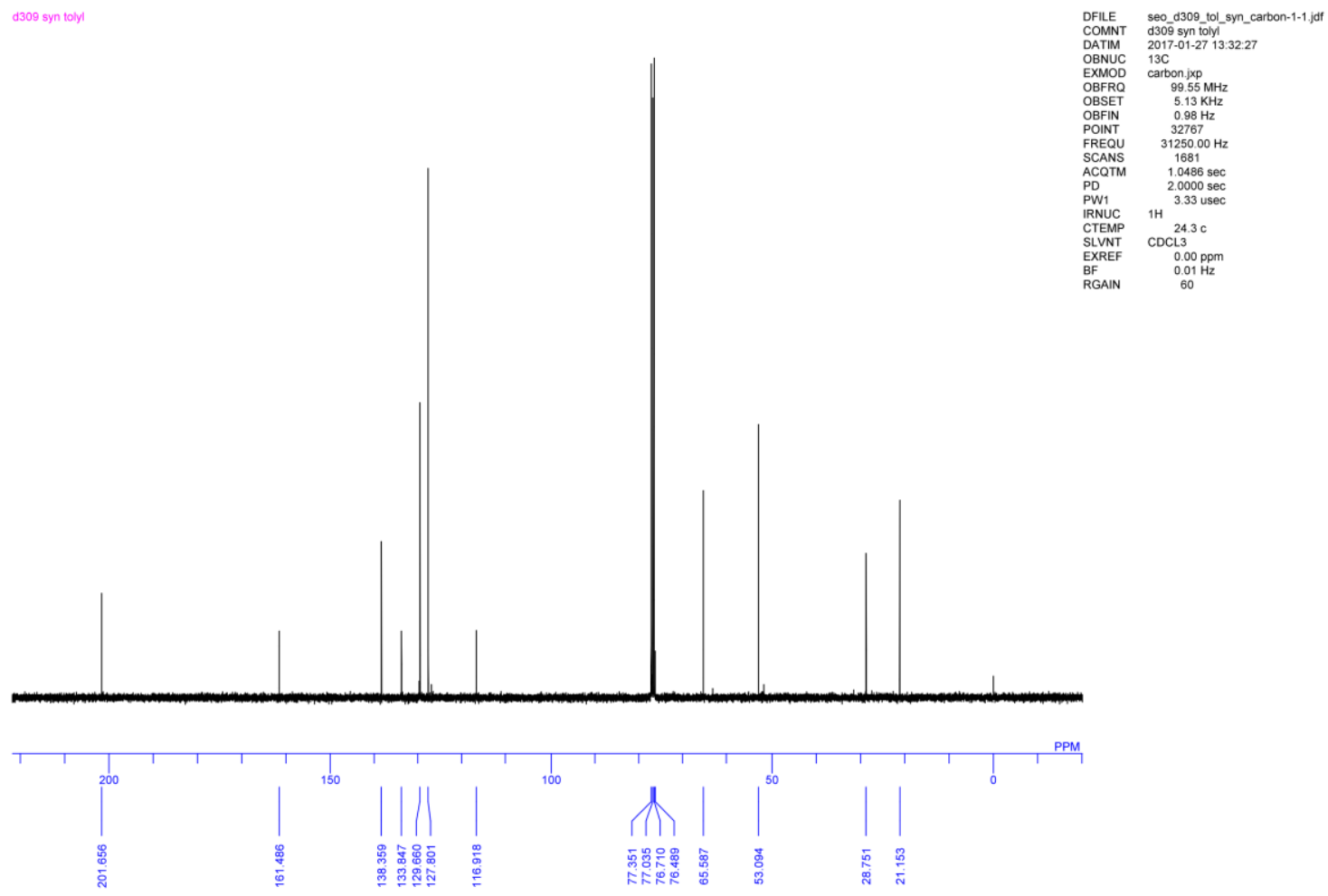


5-Acetyl-2-amino-4-(4-methoxyphenyl)-4,5-dihydrothiophene-3-carbonitrile (trans-3ad)<smiles>COc1ccc([C@H]2C(C#N)=C(N)SC2C(C)=O)cc1</smiles>

${ }^{1} \mathrm{H}$ NMR

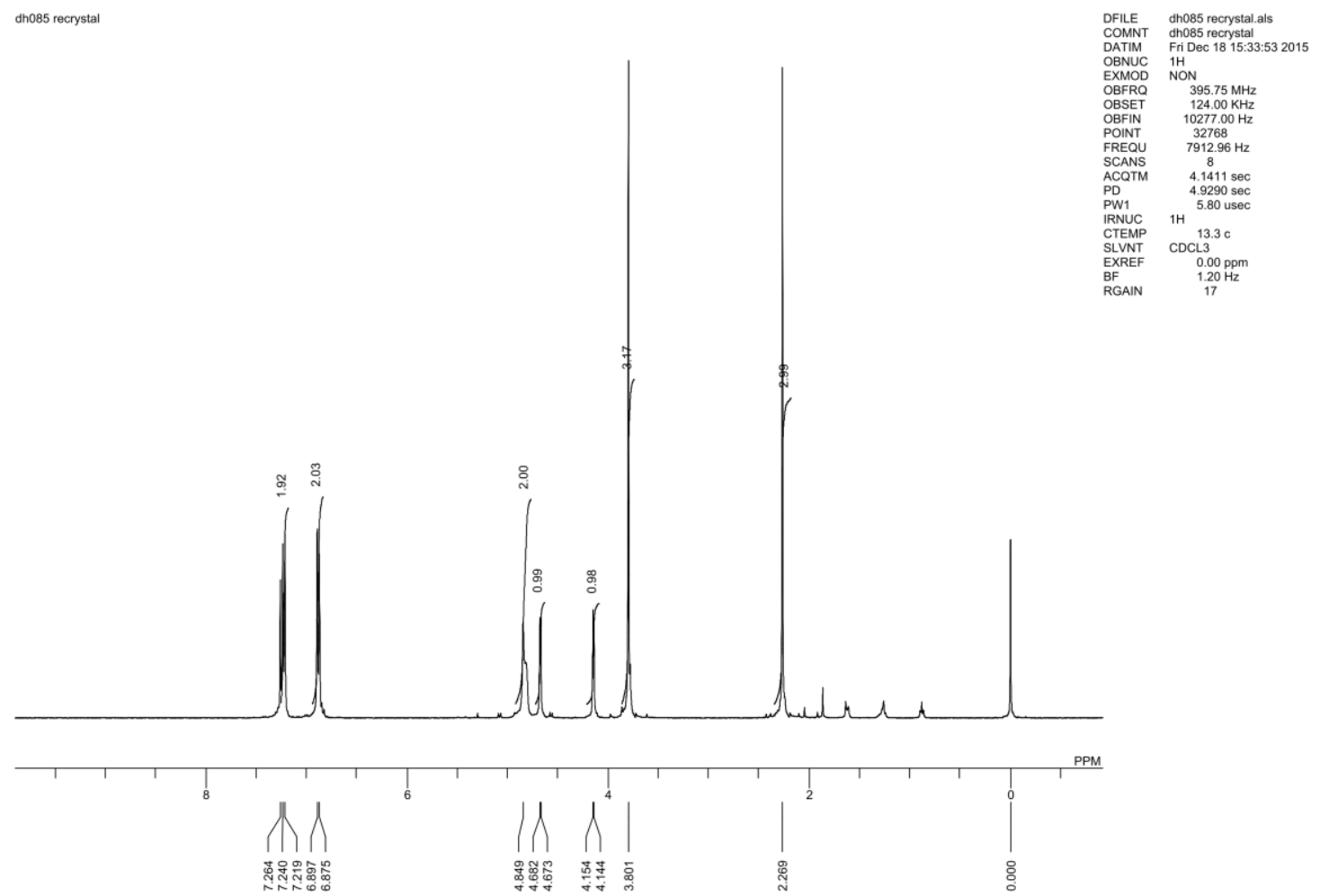


${ }^{13} \mathrm{C}$ NMR

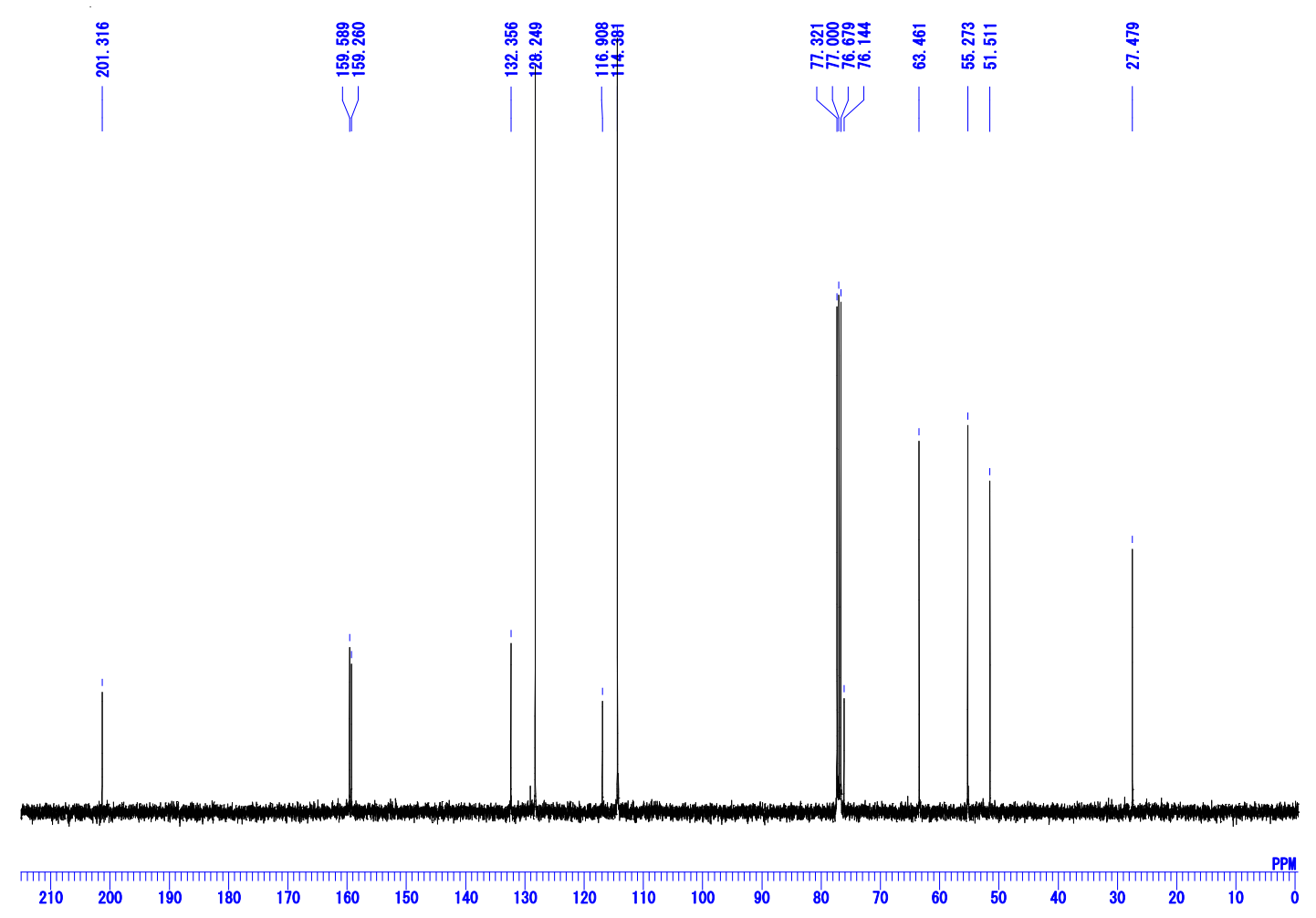

MW Profile

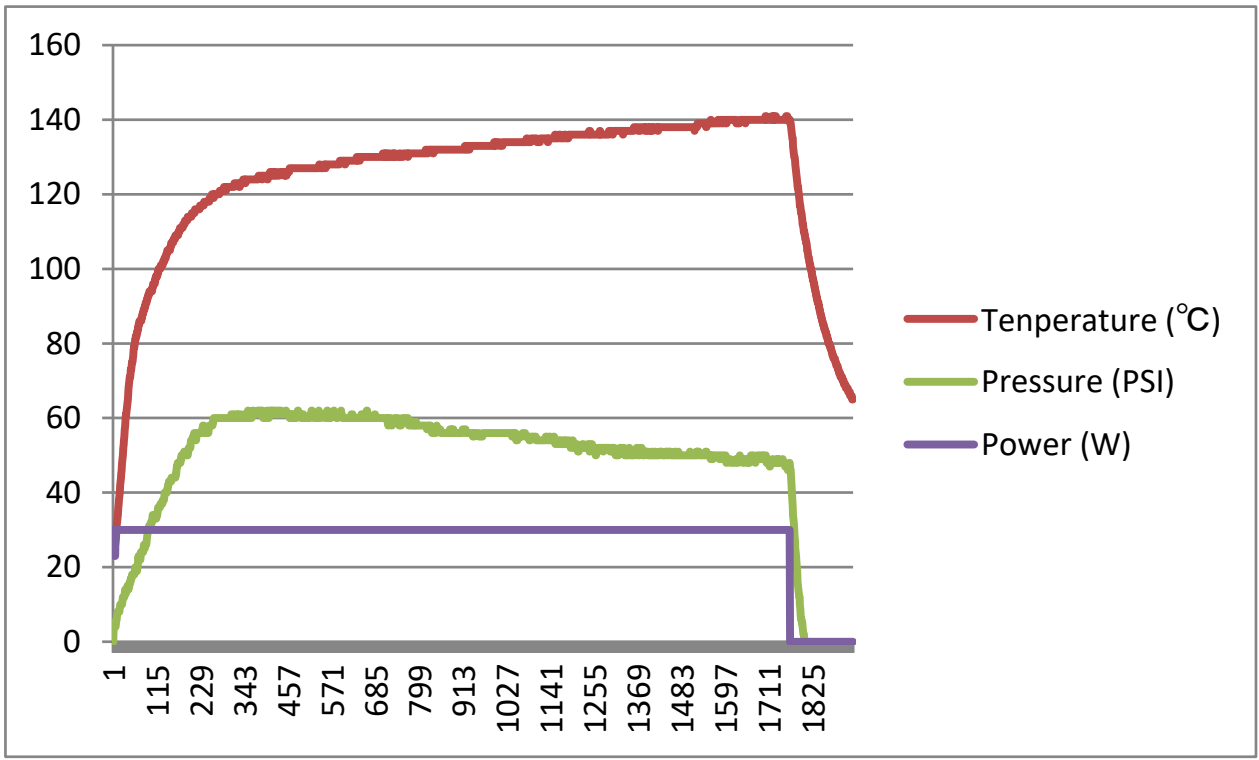


5-Acetyl-2-amino-4-(4-methoxyphenyl)-4,5-dihydrothiophene-3-carbonitrile (cis-3ad)<smiles>COc1ccc(C2C(C#N)=C(N)S[C@H]2C(C)=O)cc1</smiles>

\section{${ }^{1} \mathrm{H}$ NMR}

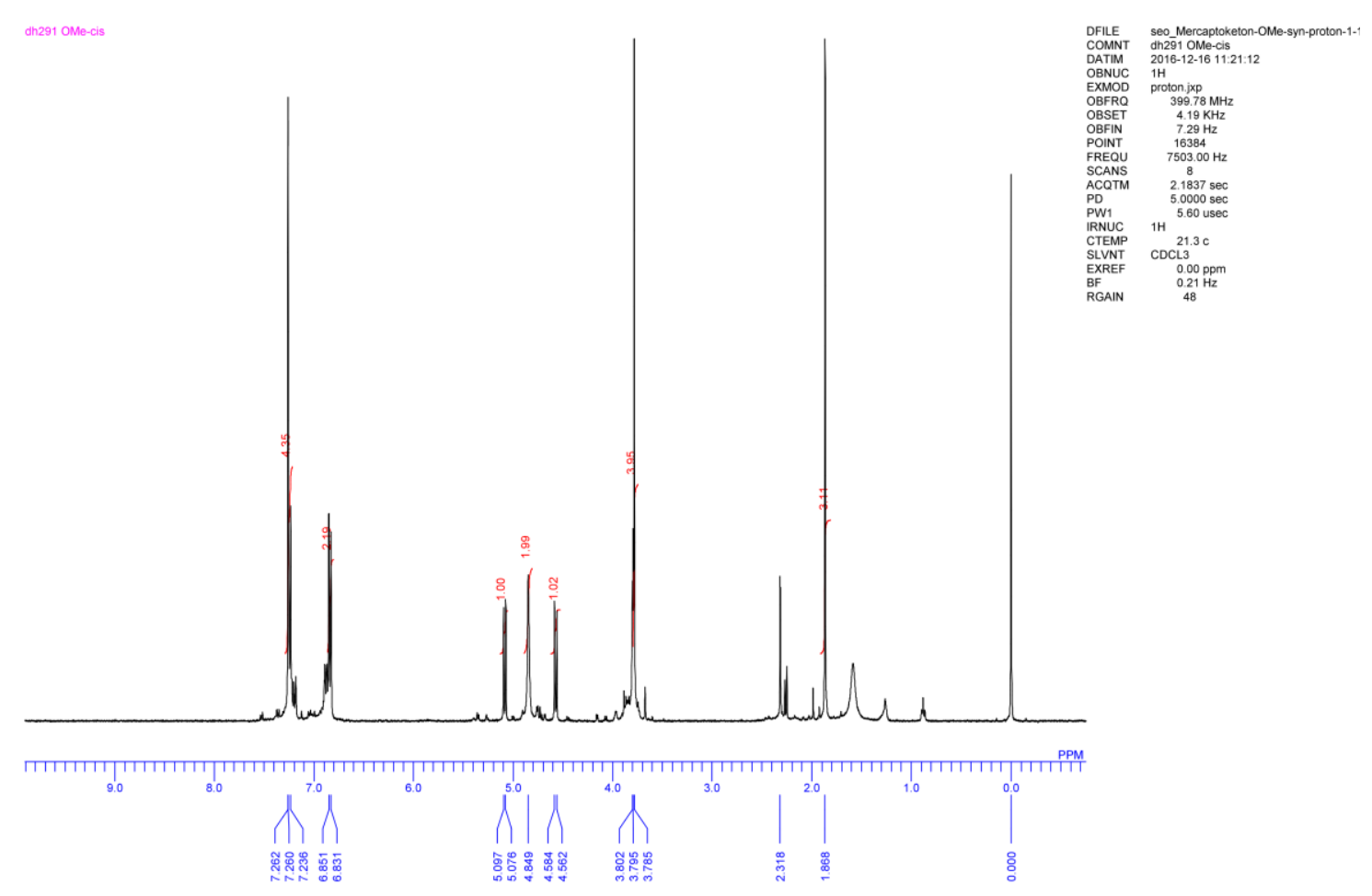


${ }^{13} \mathrm{C}$ NMR

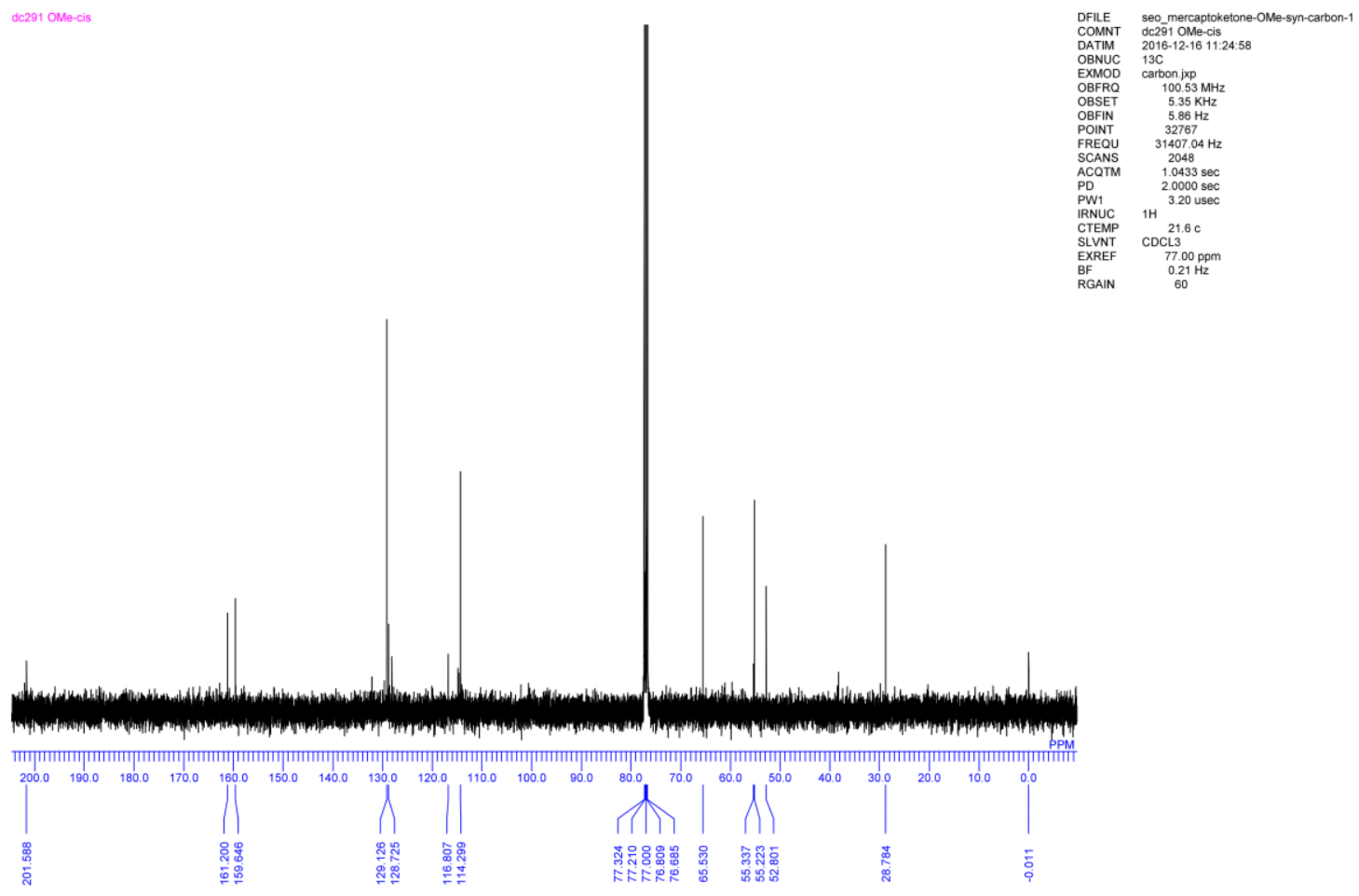


5-Acetyl-2-amino-4-(naphthalen-1-yl)-4,5-dihydrothiophene-3-carbonitrile (trans-3ae)<smiles>CC(=O)C1SC(N)=C(C#N)[C@H]1c1cccc2ccccc12</smiles>

${ }^{1} \mathrm{H}$ NMR

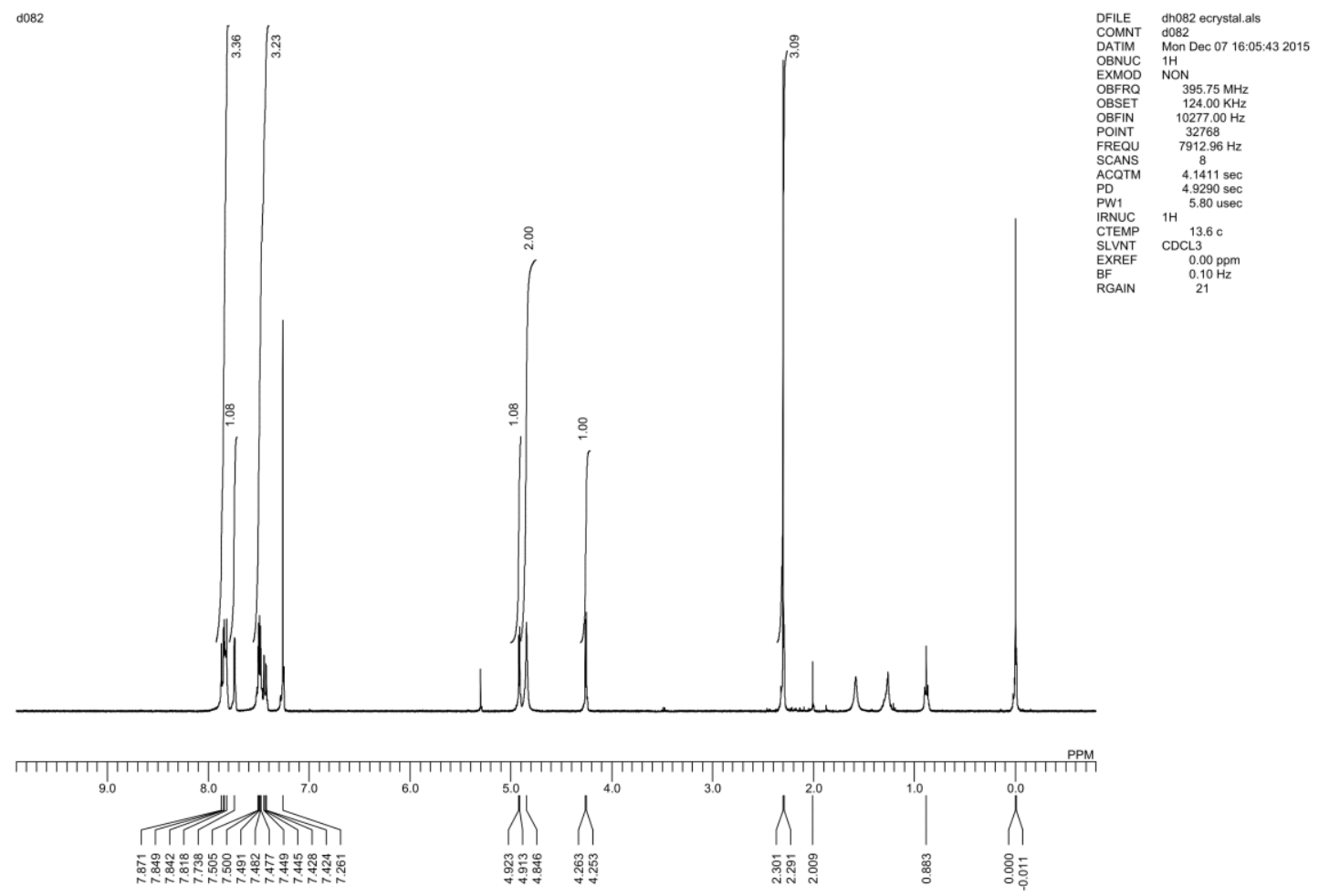


${ }^{13} \mathrm{C}$ NMR

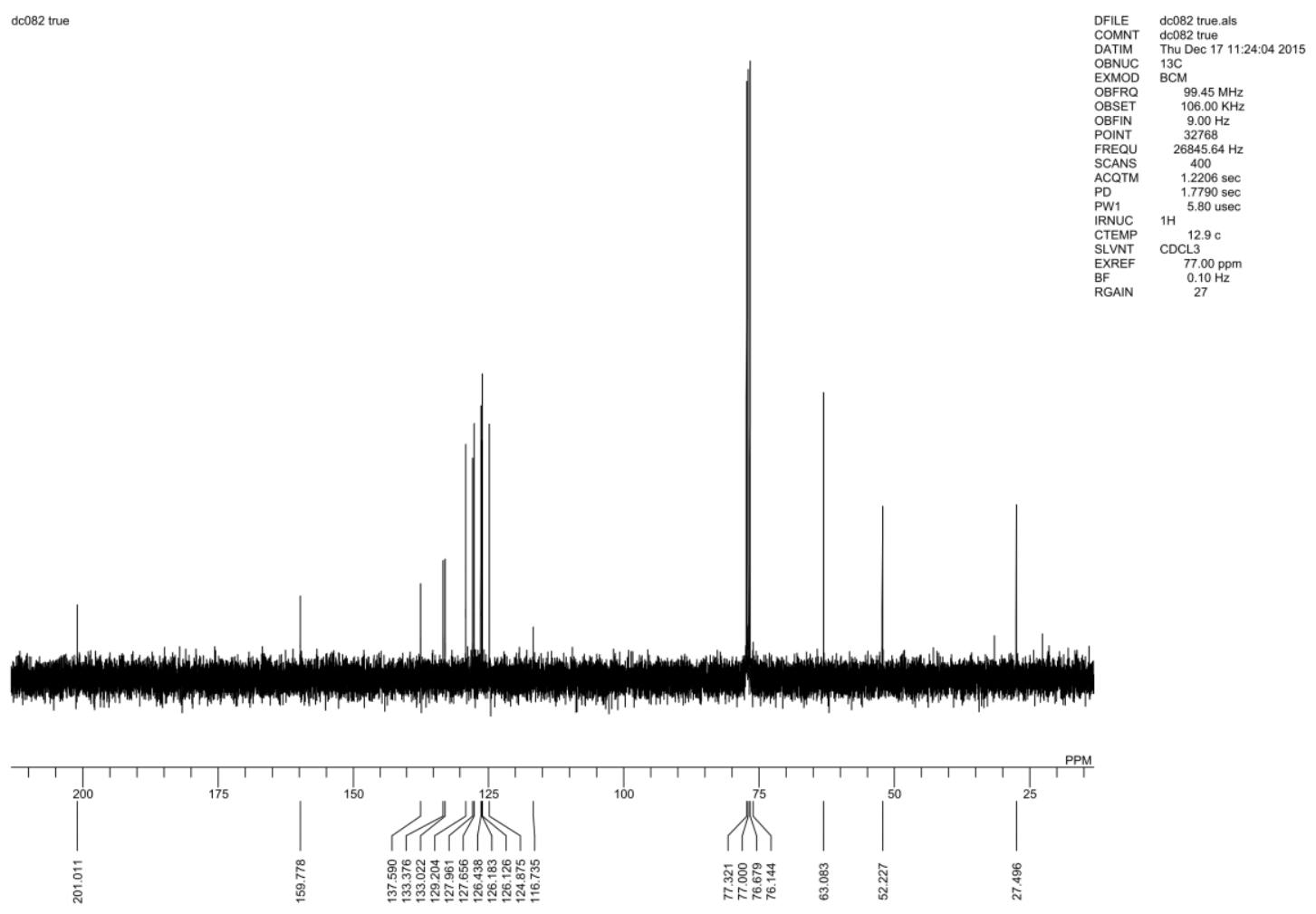

MW profile

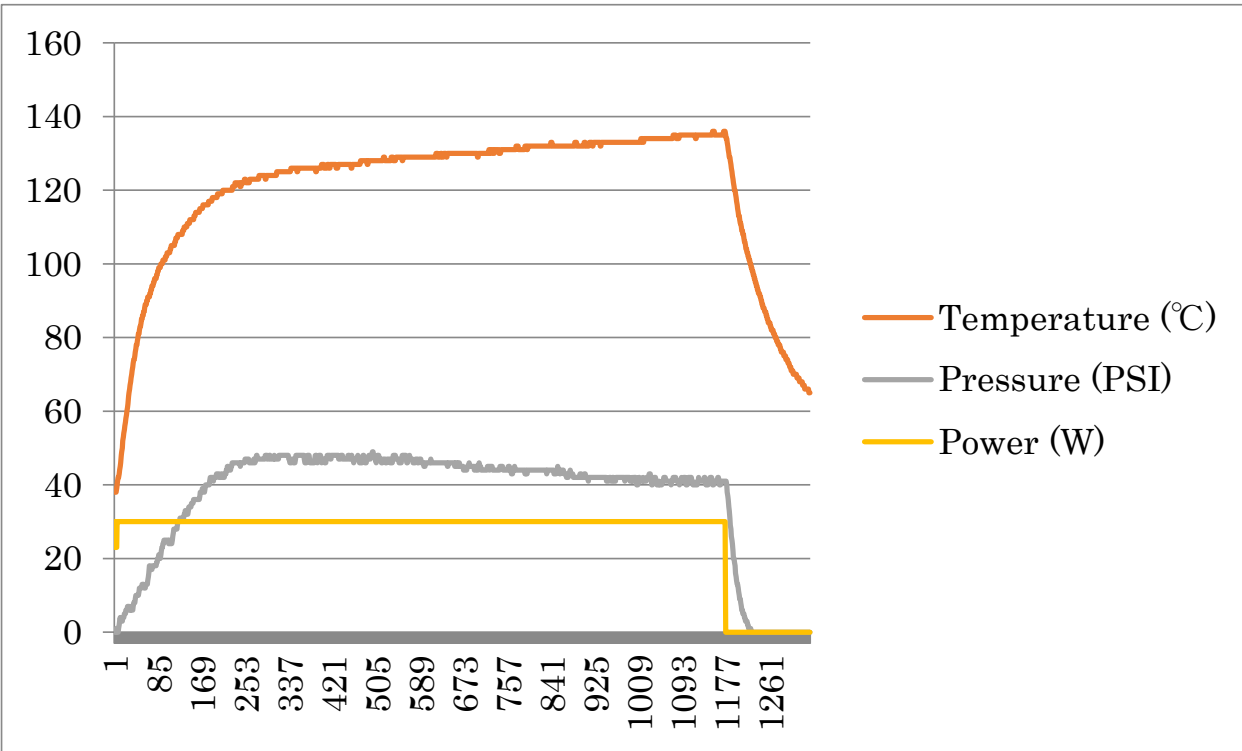


5-Acetyl-2-amino-4-(naphthalen-1-yl)-4,5-dihydrothiophene-3-carbonitrile (cis-3ae)

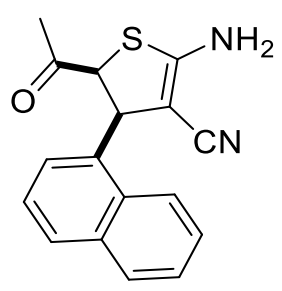

${ }^{1} \mathrm{H}$ NMR

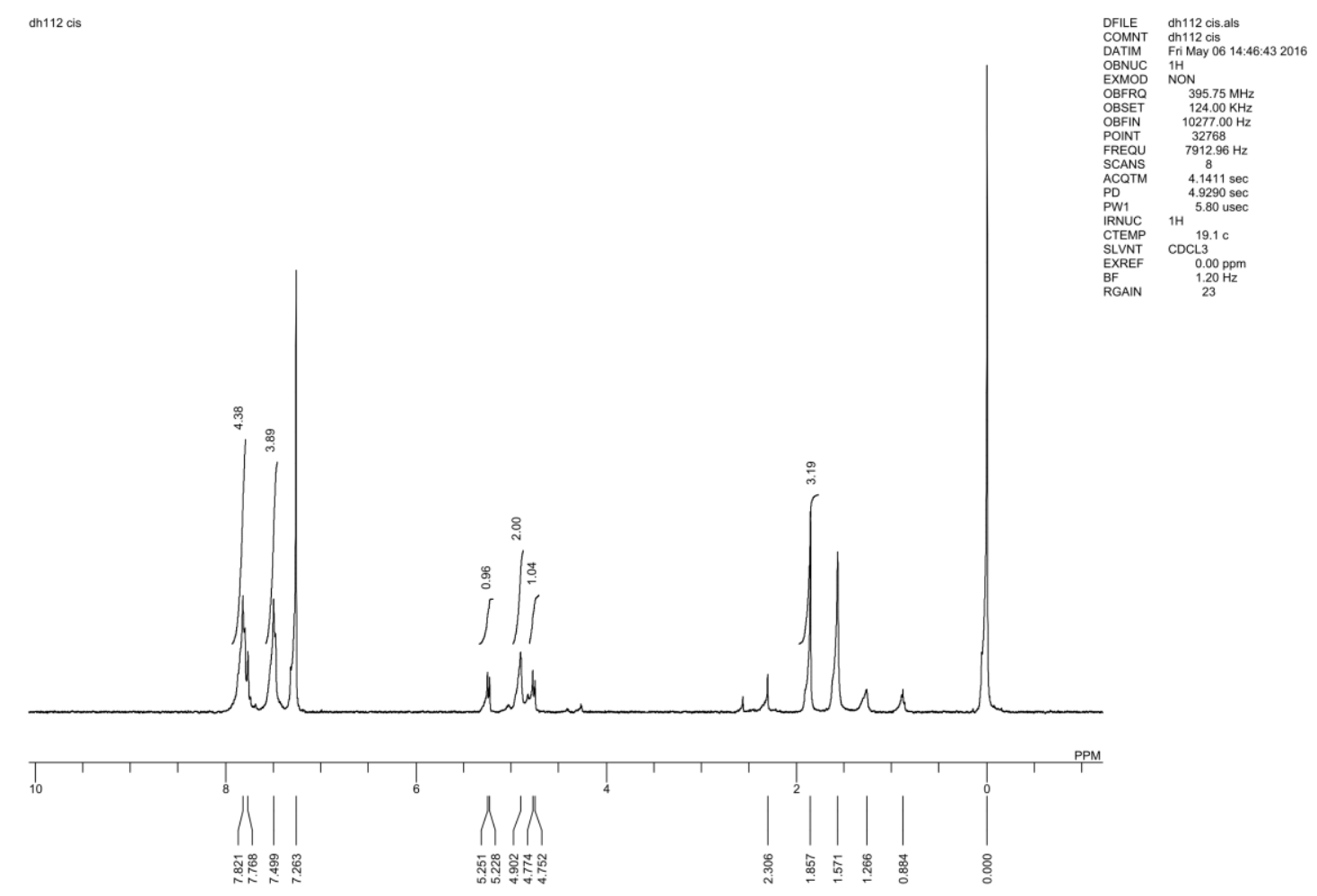


${ }^{13} \mathrm{C}$ NMR

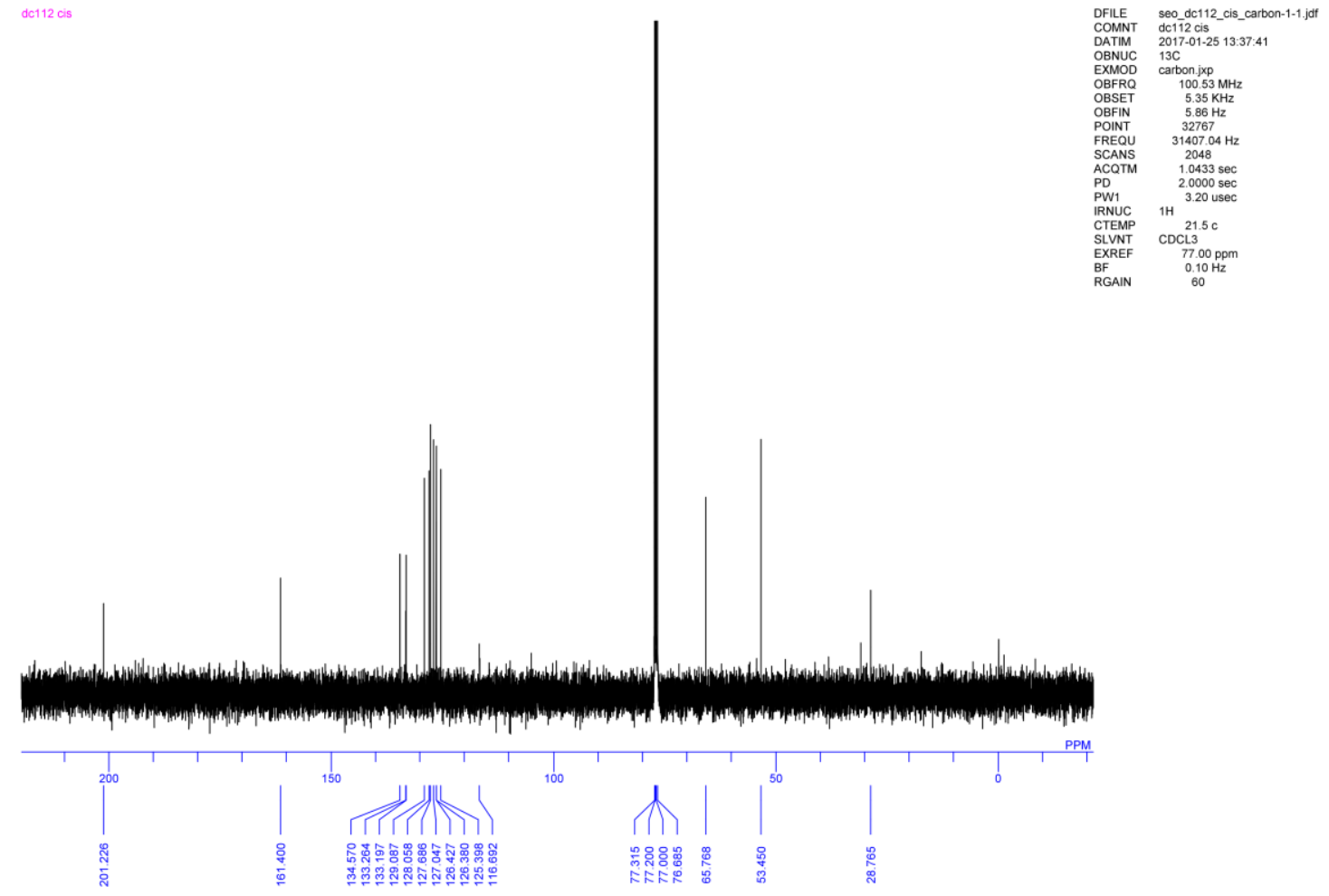


5-Acetyl-2-amino-4-(furan-2-yl)-4,5-dihydrothiophene-3-carbonitrile (trans-3af)<smiles>CC(=O)C1SC(N)=C(C#N)[C@H]1c1ccco1</smiles>

${ }^{1} \mathrm{H}$ NMR

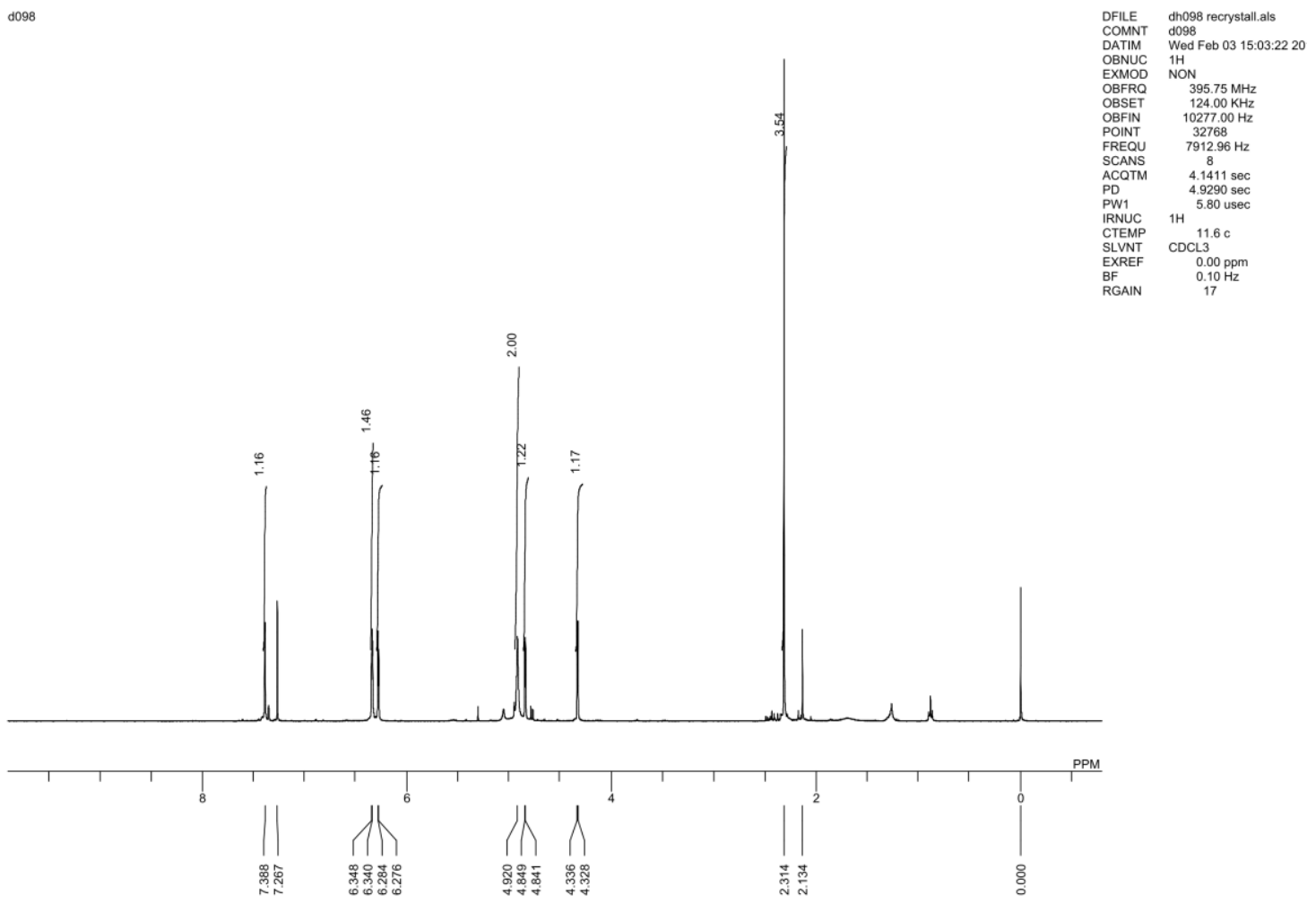


${ }^{13} \mathrm{C}$ NMR

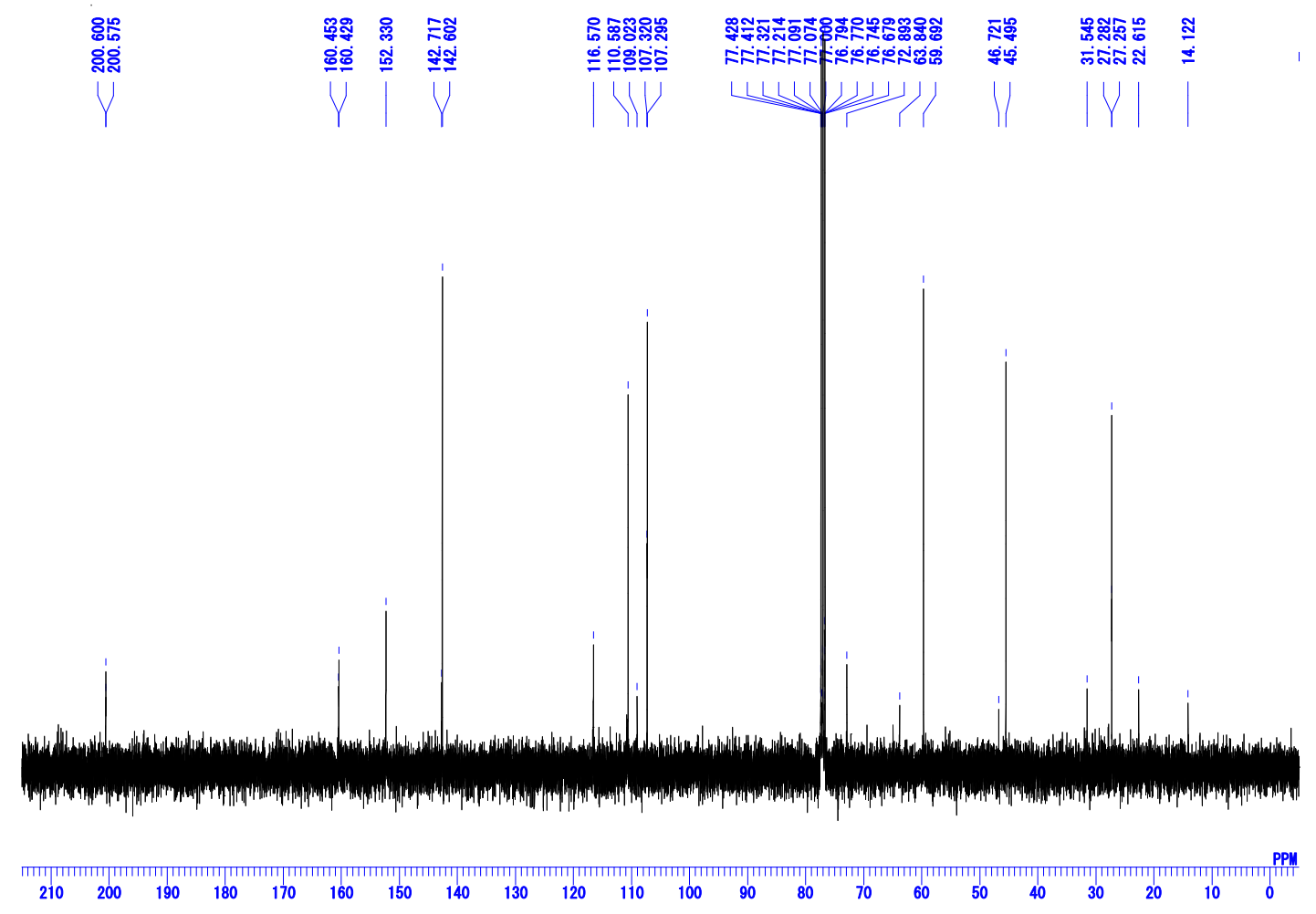

MW Profile

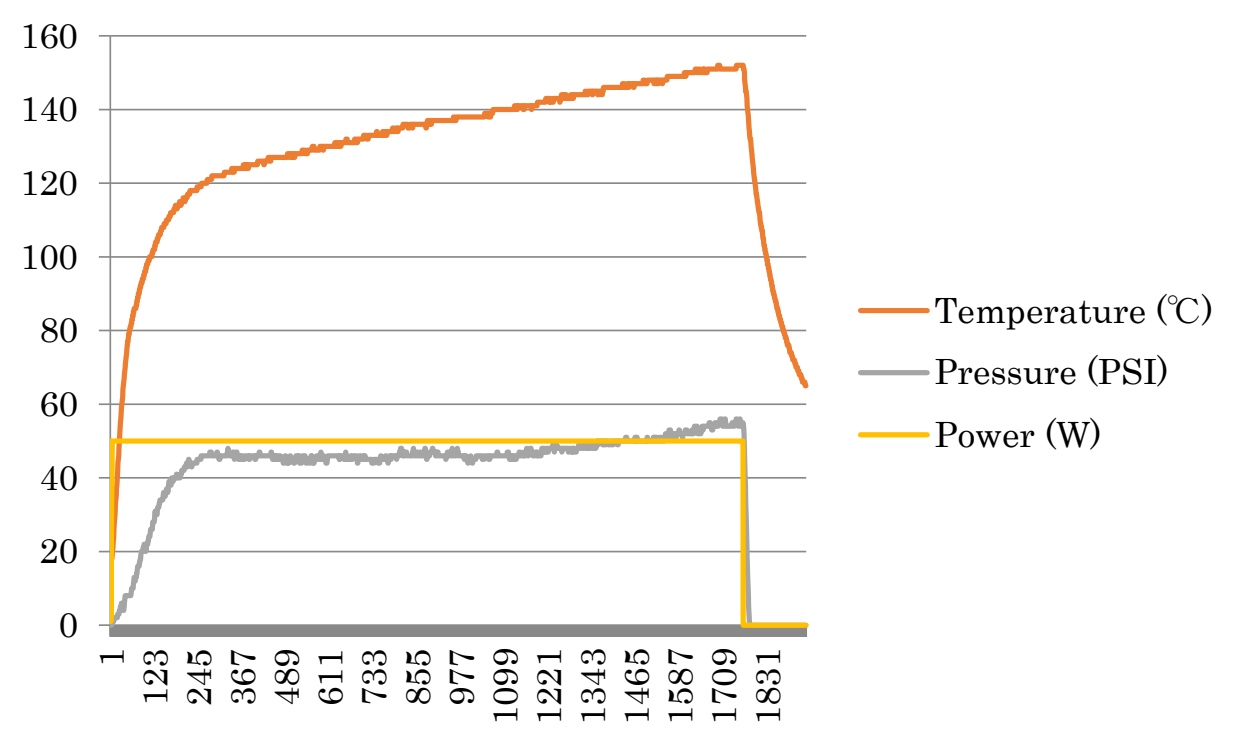


5-Acetyl-2-amino-4-(furan-2-yl)-4,5-dihydrothiophene-3-carbonitrile (cis-3af)<smiles>CC(=O)C1SC(N)=C(C#N)C1c1ccco1</smiles>

${ }^{1} \mathrm{H}$ NMR

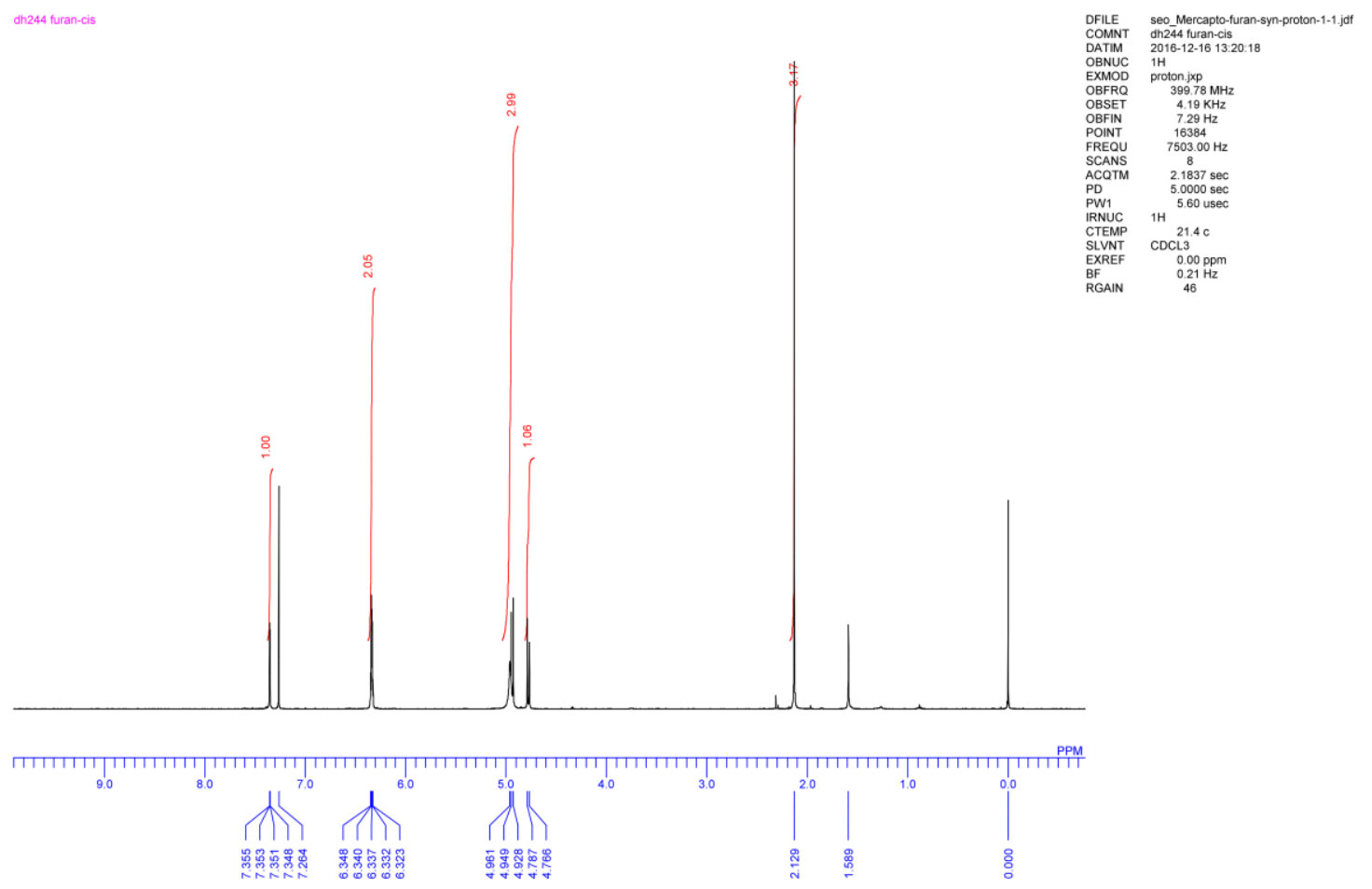


${ }^{13} \mathrm{C}$ NMR

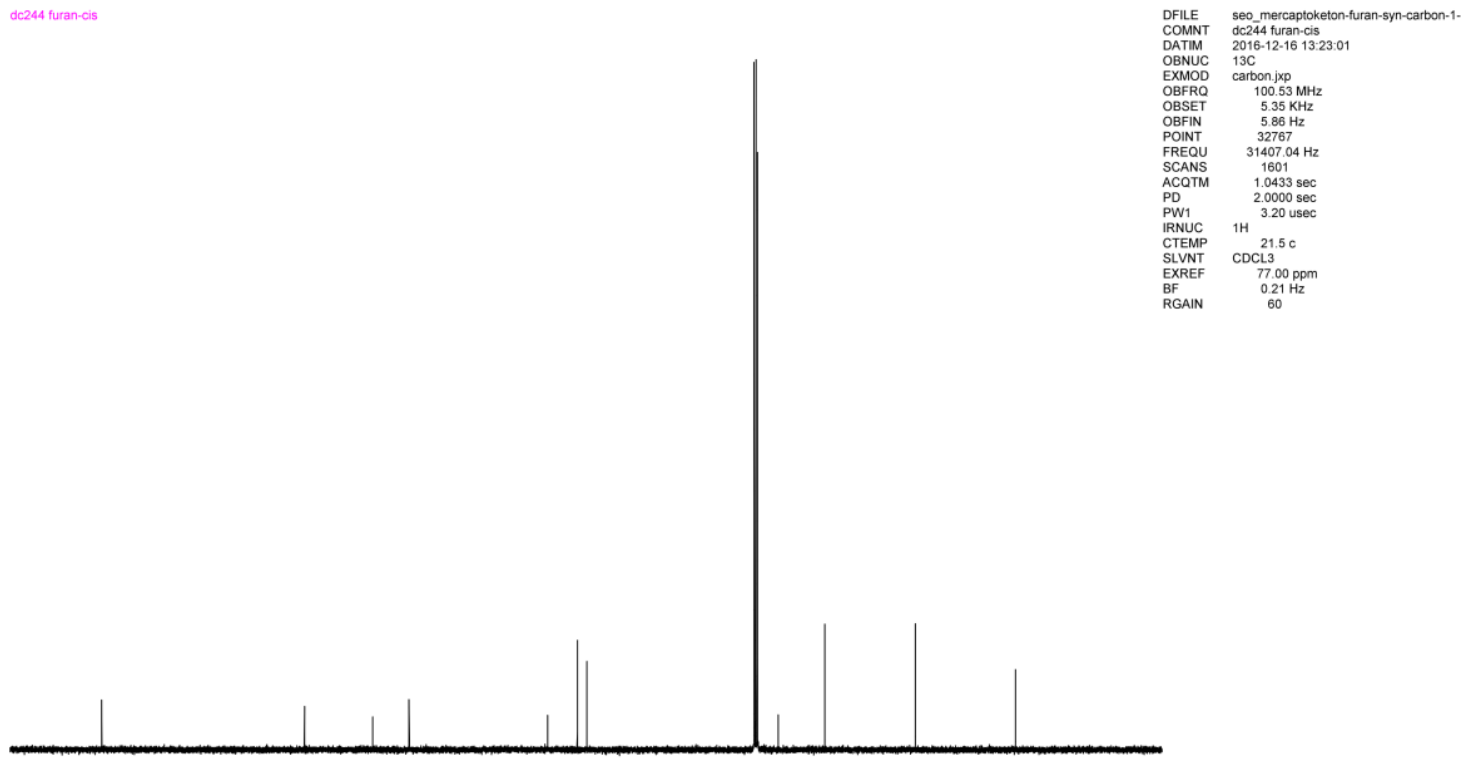

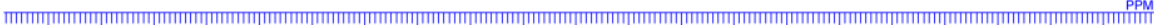

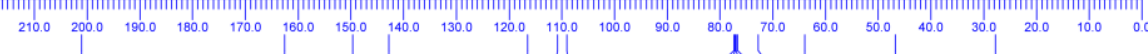

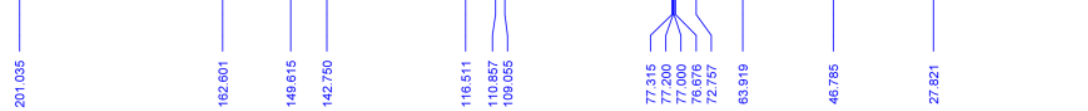


5-Acetyl-2-amino-4-phenethyl-4,5-dihydrothiophene-3-carbonitrile (trans-3ag)<smiles>CC(=O)C1SC(N)=C(C#N)[C@H]1CCc1ccccc1</smiles>

${ }^{1} \mathrm{H}$ NMR

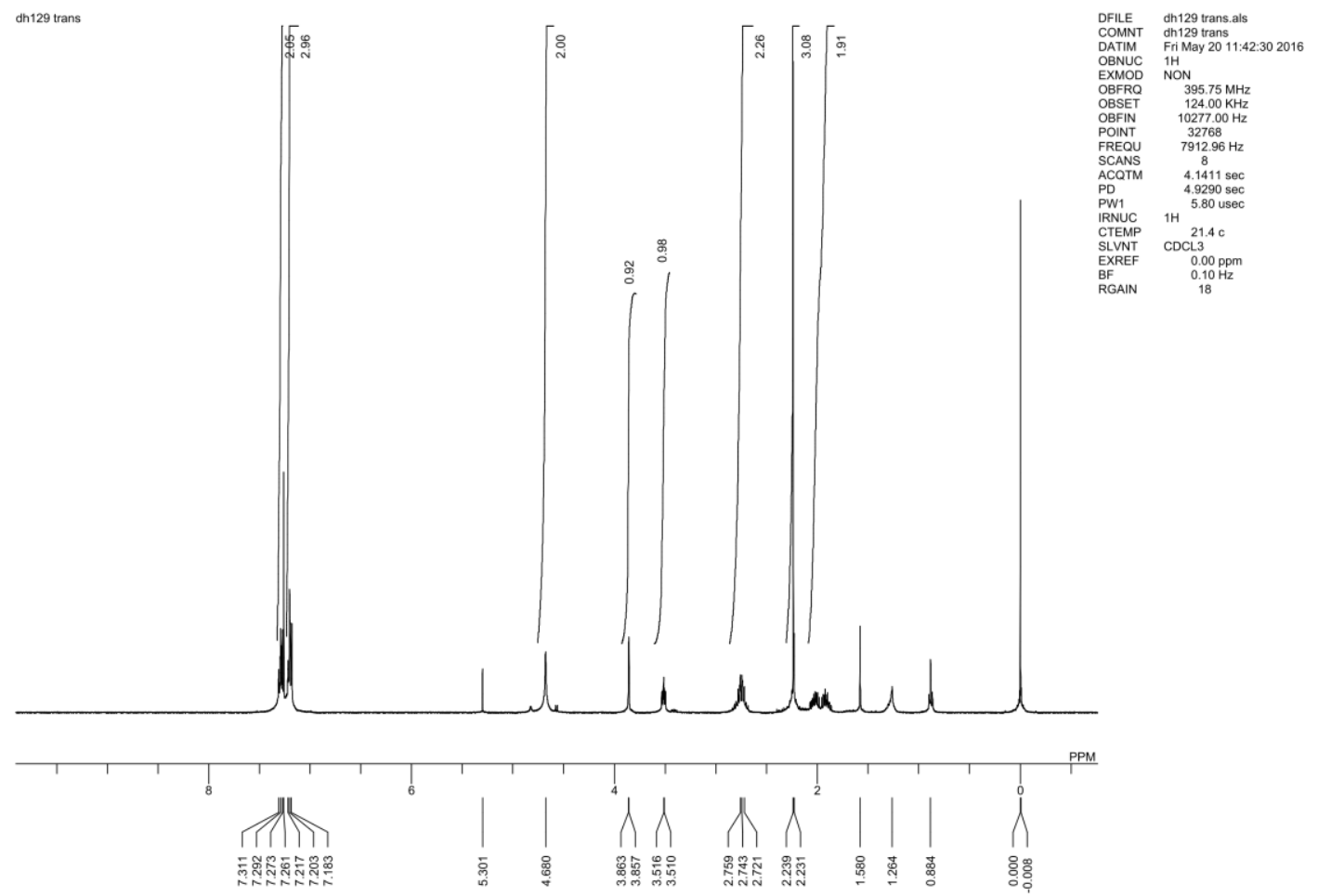


${ }^{13} \mathrm{C}$ NMR

dc 128 trans

DFLLE dc128 trans.al

$\begin{array}{lll}\text { COMNT } & \text { de } 128 \text { trans } \\ \text { DATIM } & \text { Fri May } 20 \text { 16:44:13 } 20\end{array}$

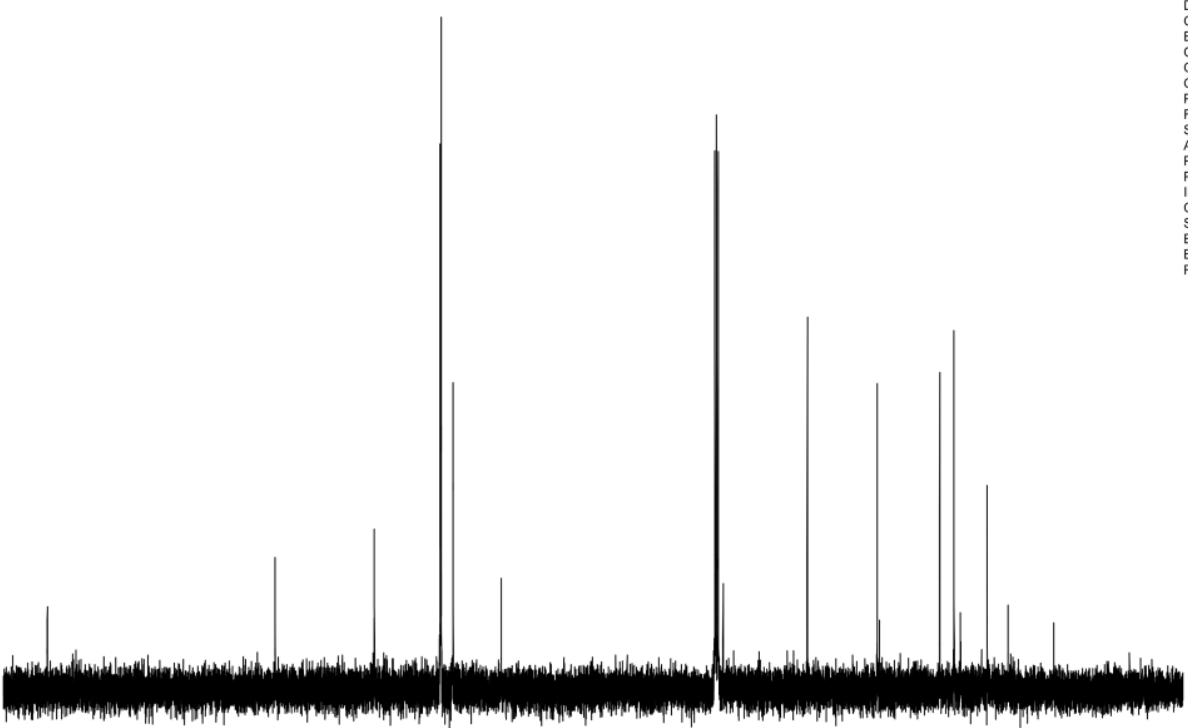

$\begin{array}{ll}99.45 \mathrm{MHz} \\ \text { OBSET } & 106.00 \mathrm{KHz}\end{array}$

$\begin{array}{ll}9.00 \mathrm{~Hz} \\ \mathrm{OBF} \\ \text { PINT } & 32768 \\ & \end{array}$

$\begin{array}{lr}\text { SCANS } & 600 \\ \text { ACQTM } & 1.2206 \mathrm{sec}\end{array}$

PW1 $\begin{array}{ll}1.7790 \mathrm{sec} \\ 5.80 \mathrm{usec}\end{array}$

TIEMP 21.20

$\begin{array}{ll}\text { EXREF } & 77.00 \mathrm{ppm} \\ \mathrm{BF} & 0.10 \mathrm{~Hz}\end{array}$

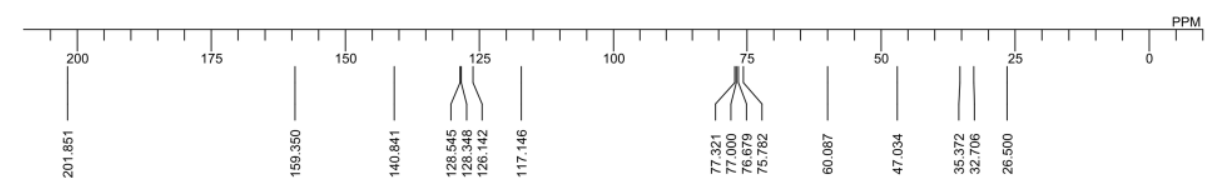

MW profile

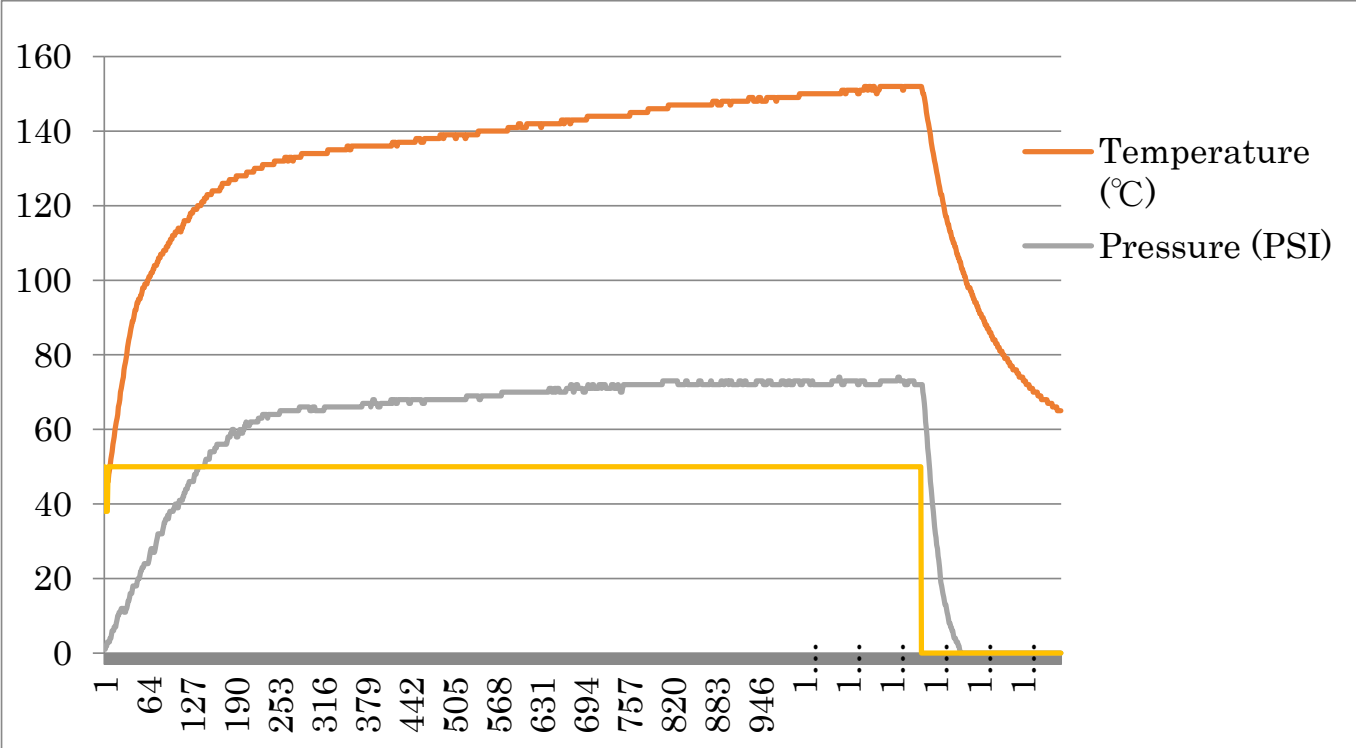


5-Acetyl-2-amino-4-phenethyl-4,5-dihydrothiophene-3-carbonitrile (cis-3ag)<smiles>CC(=O)C1SC(N)=C(C#N)C1CCc1ccccc1</smiles>

${ }^{1} \mathrm{H}$ NMR

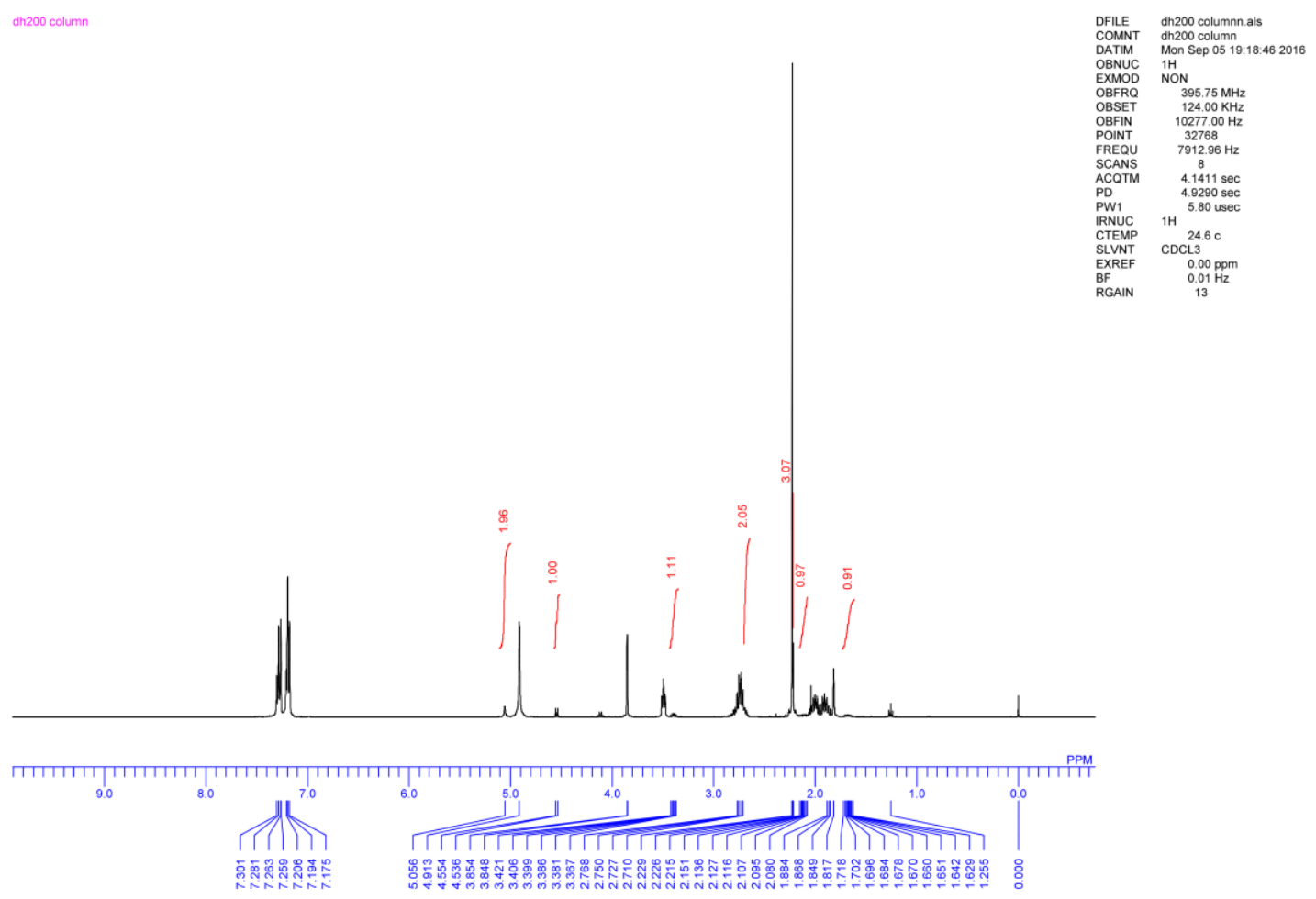


2-Amino-4-phenyl-5-pivaloyl-4,5-dihydrothiophene-3-carbonitrile (trans-3ba)<smiles>CC(C)(C)C(=O)[C@H]1SC(N)=C(C#N)[C@H]1c1ccccc1</smiles>

${ }^{1} \mathrm{HNMR}$

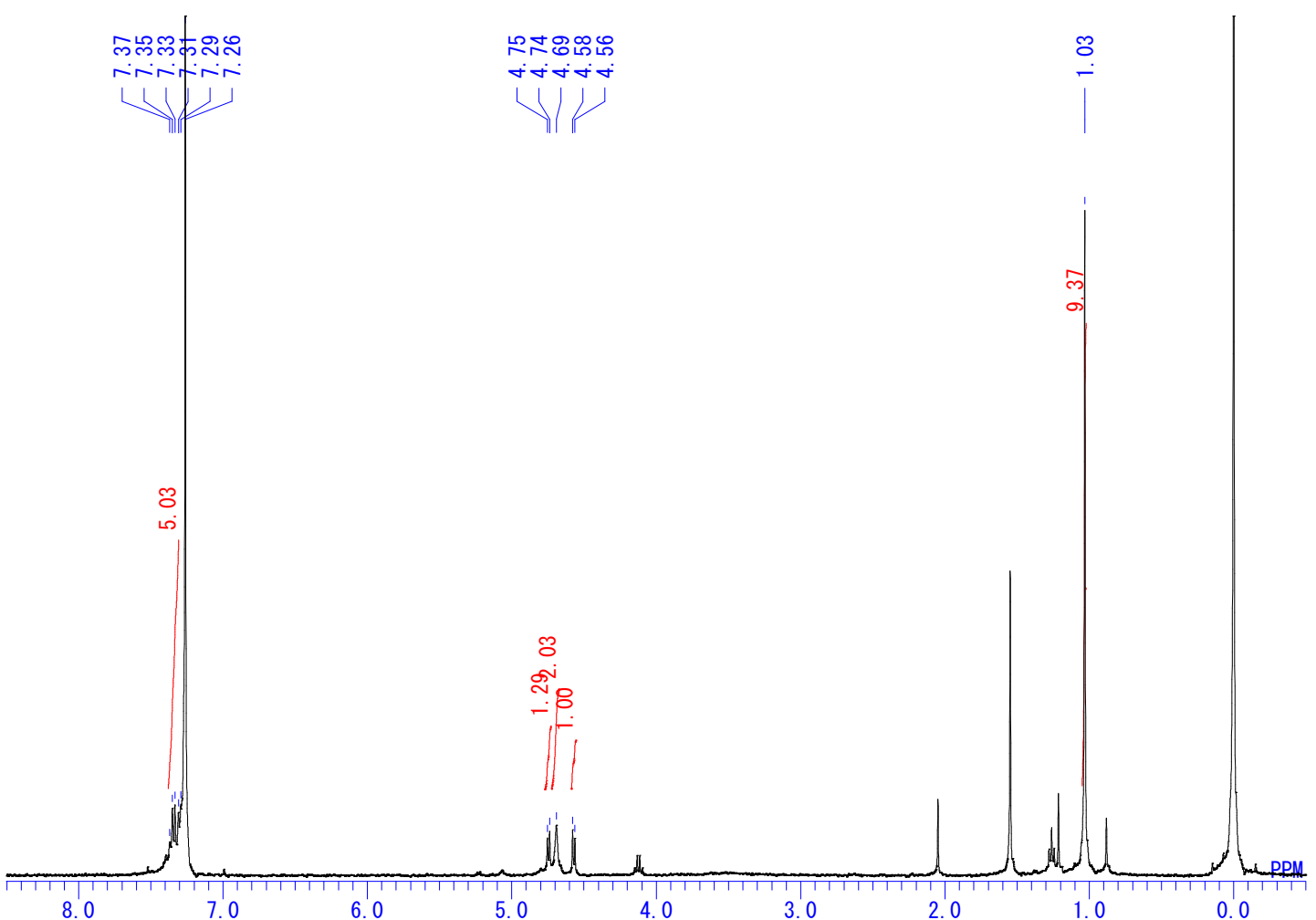


${ }^{13} \mathrm{CNMR}$

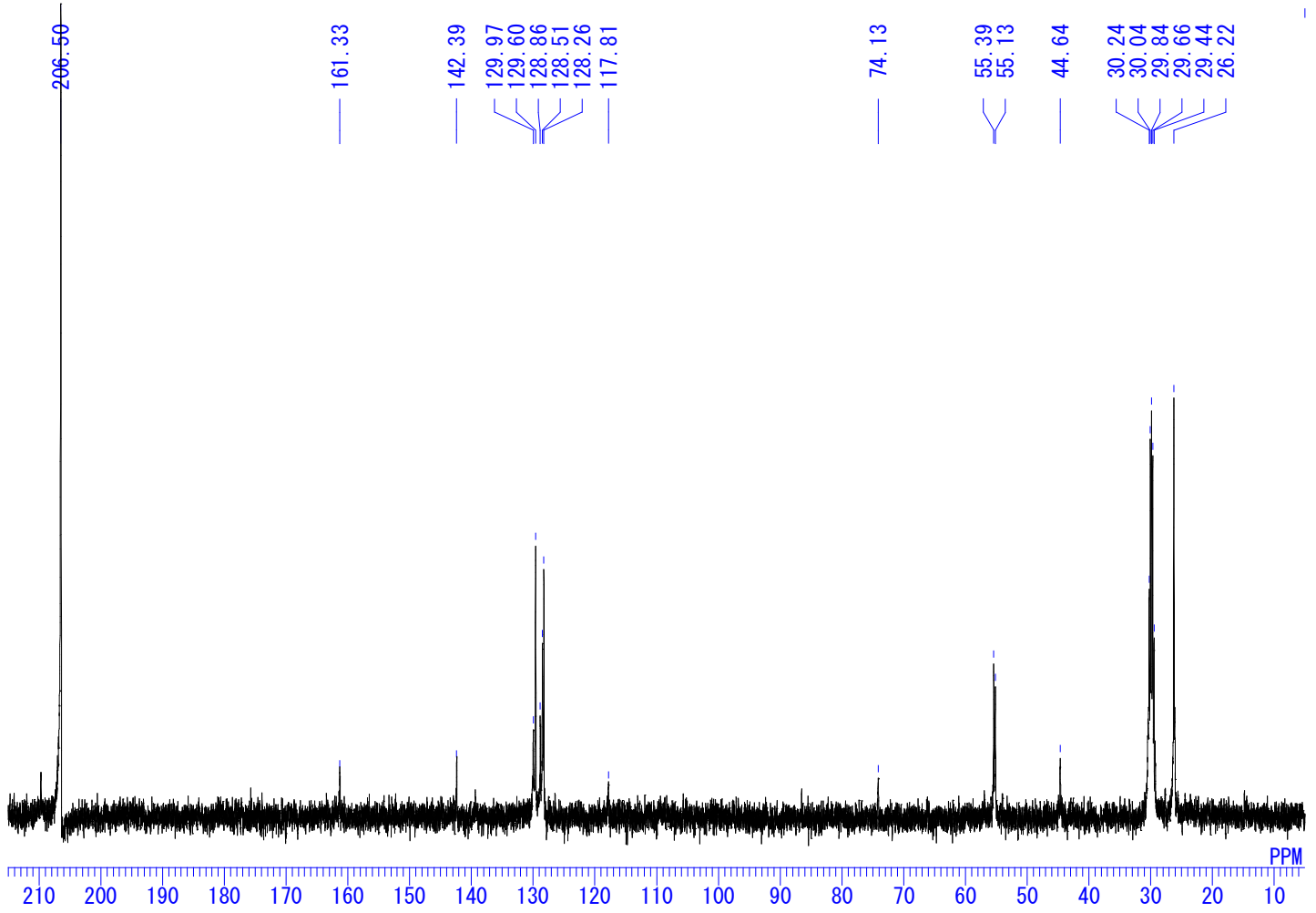

MW

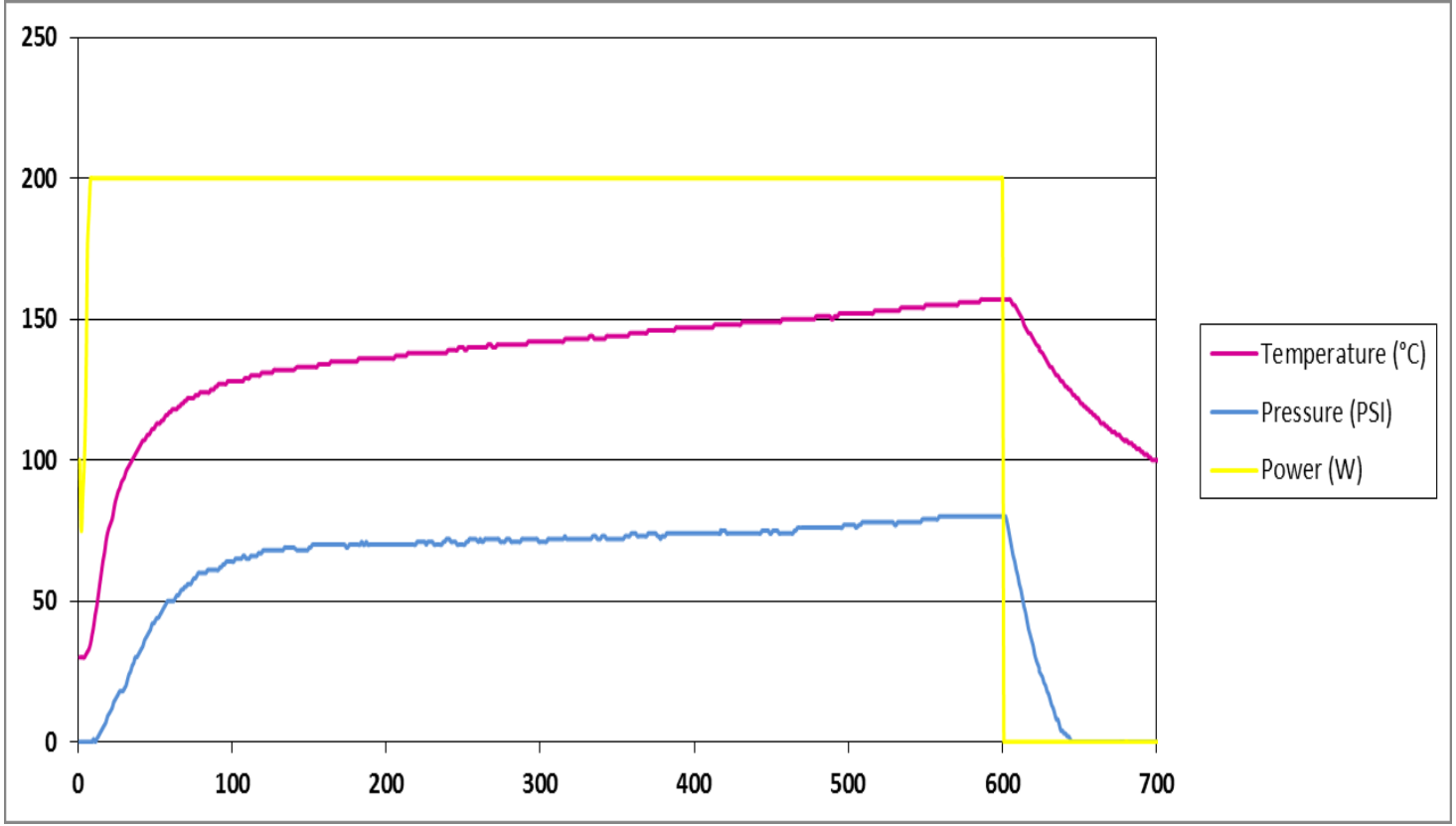


2-Amino-4-phenyl-5-pivaloyl-4,5-dihydrothiophene-3-carbonitrile (cis-3ba)

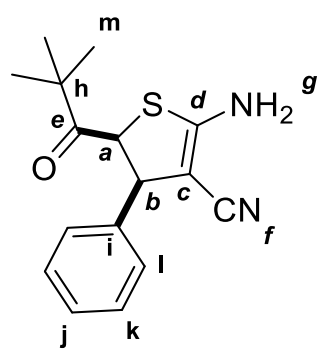

${ }^{1} \mathrm{HNMR}$

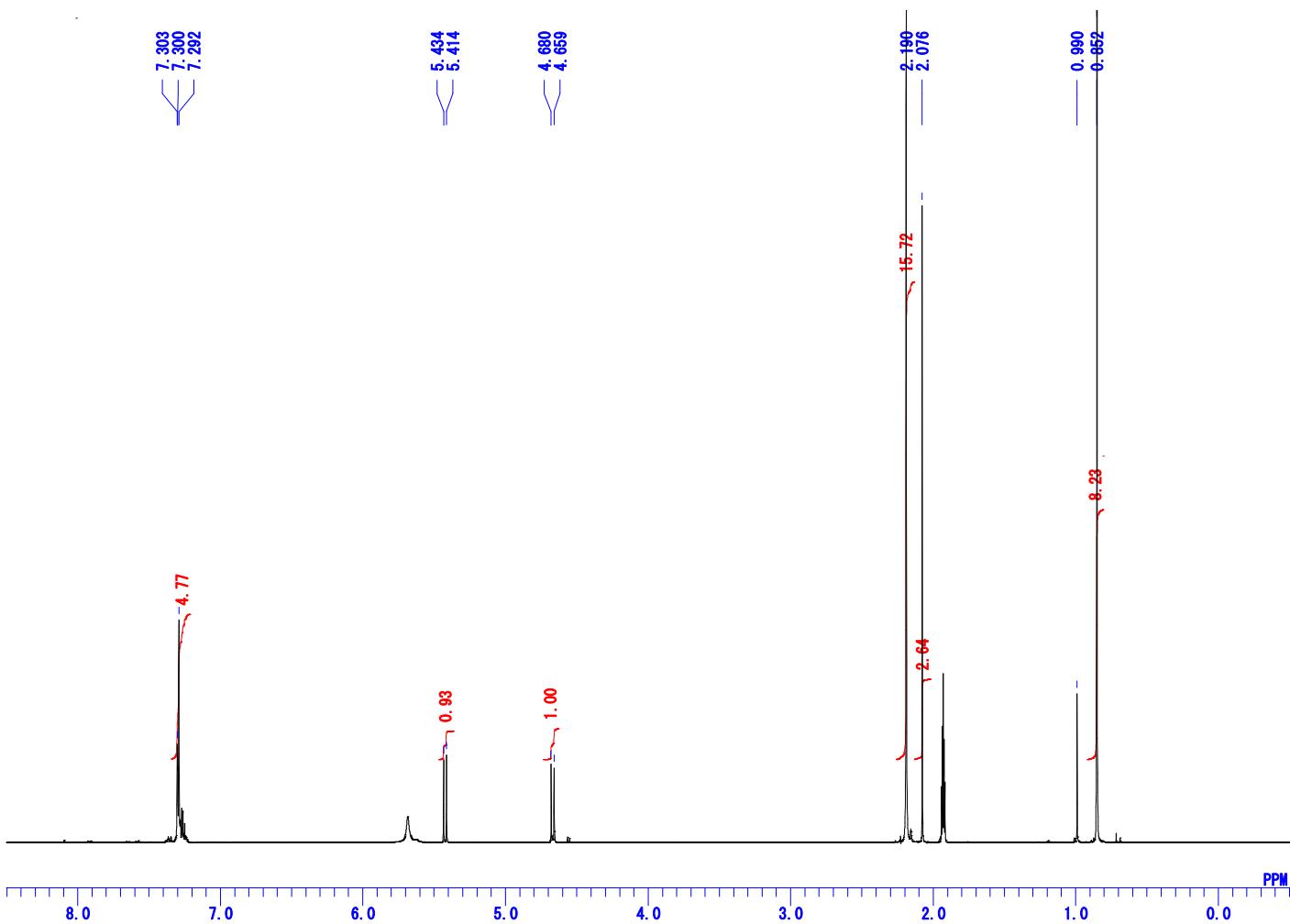


${ }^{13} \mathrm{CNMR}$

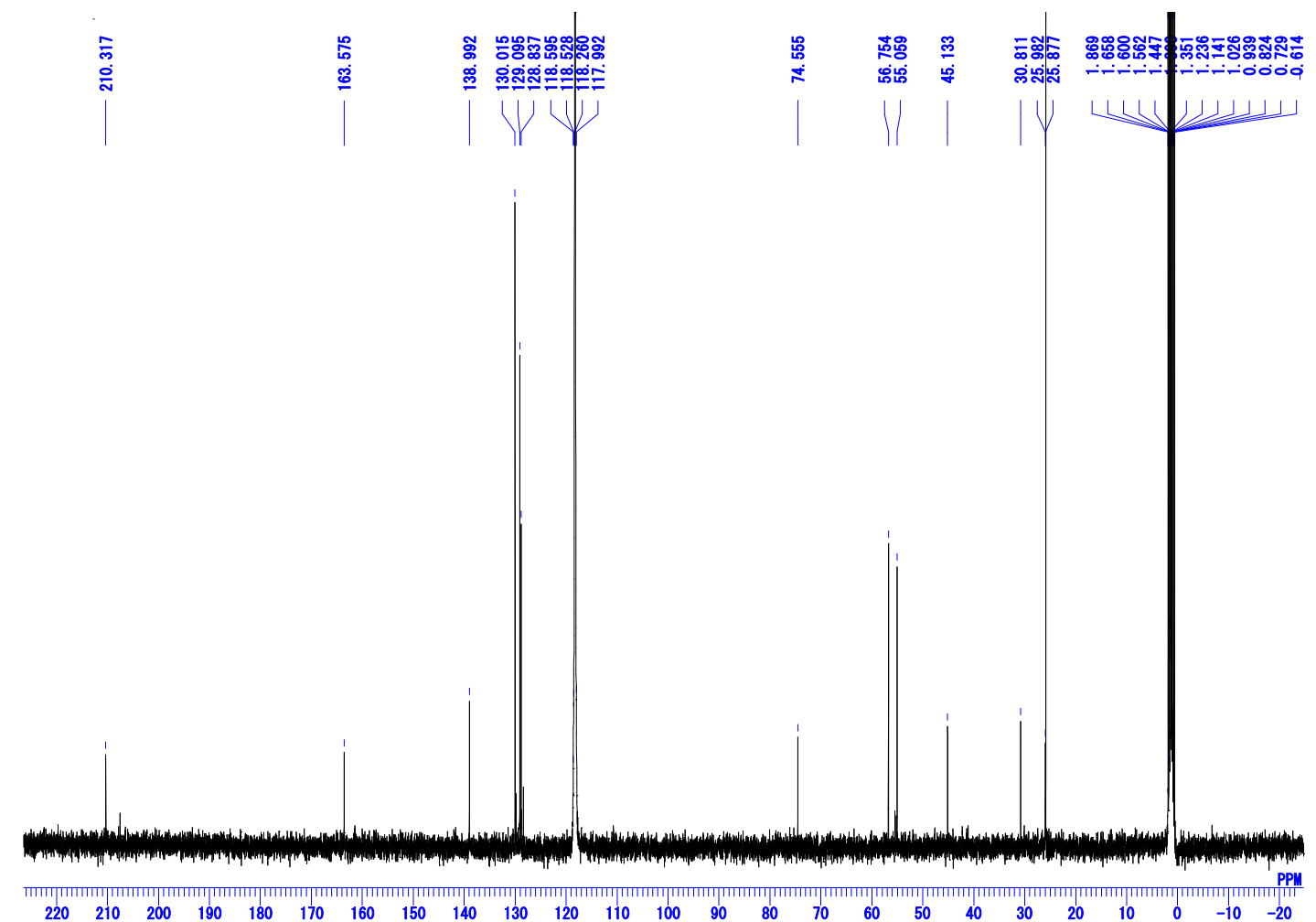

MW Profile

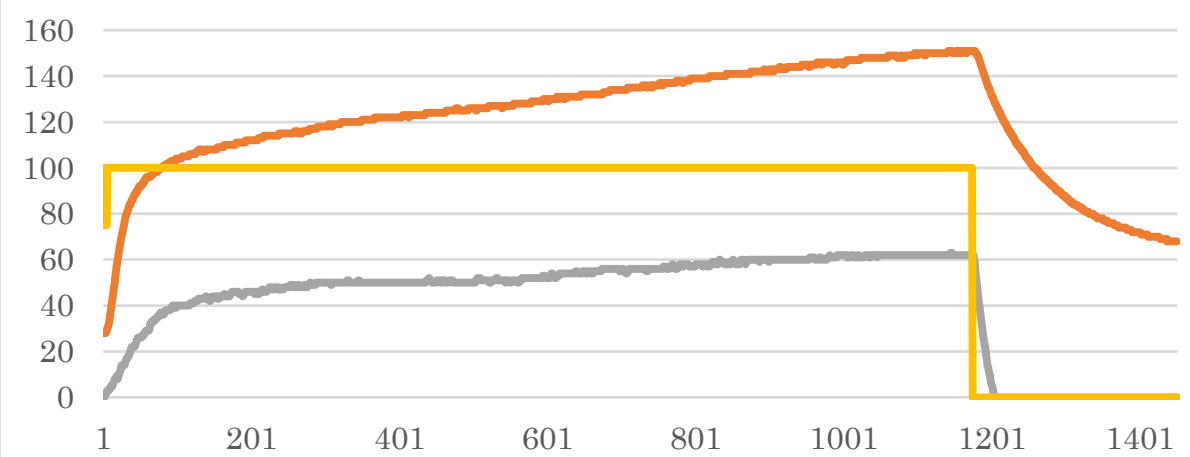

Temperature $\left({ }^{\circ} \mathrm{C}\right)$

Pressure (PSI)

Power (W) 
2-Amino-4-(4-chlorophenyl)-5-pivaloyl-4,5-dihydrothiophene-3-carbonitrile (trans-3bb)

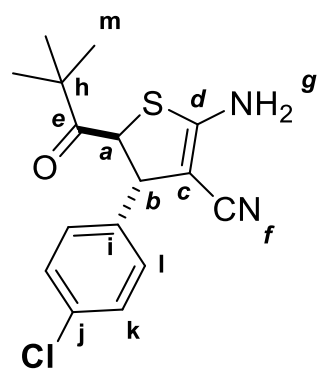

${ }^{1} \mathrm{H}$ NMR

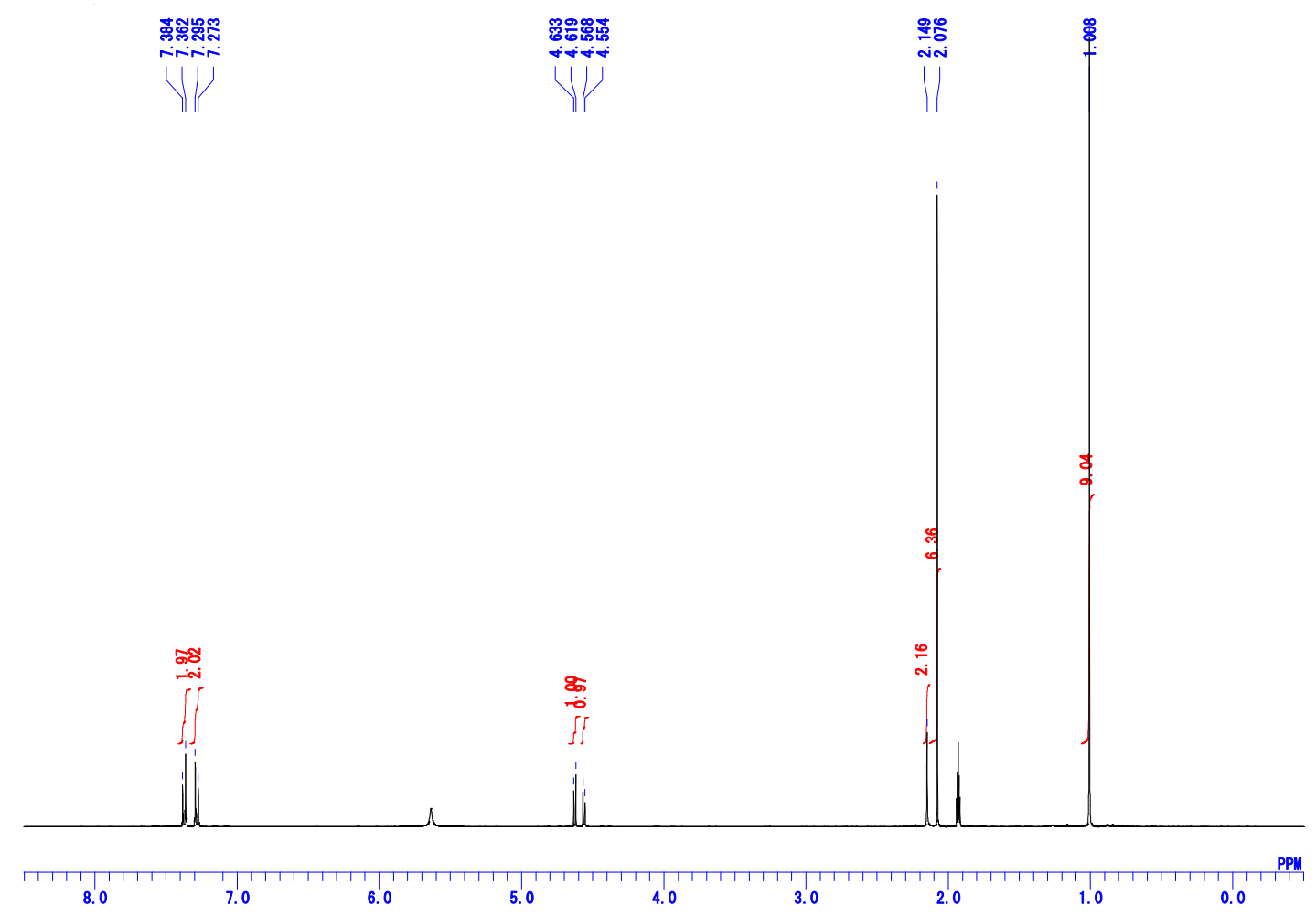


${ }^{13} \mathrm{C}$ NMR

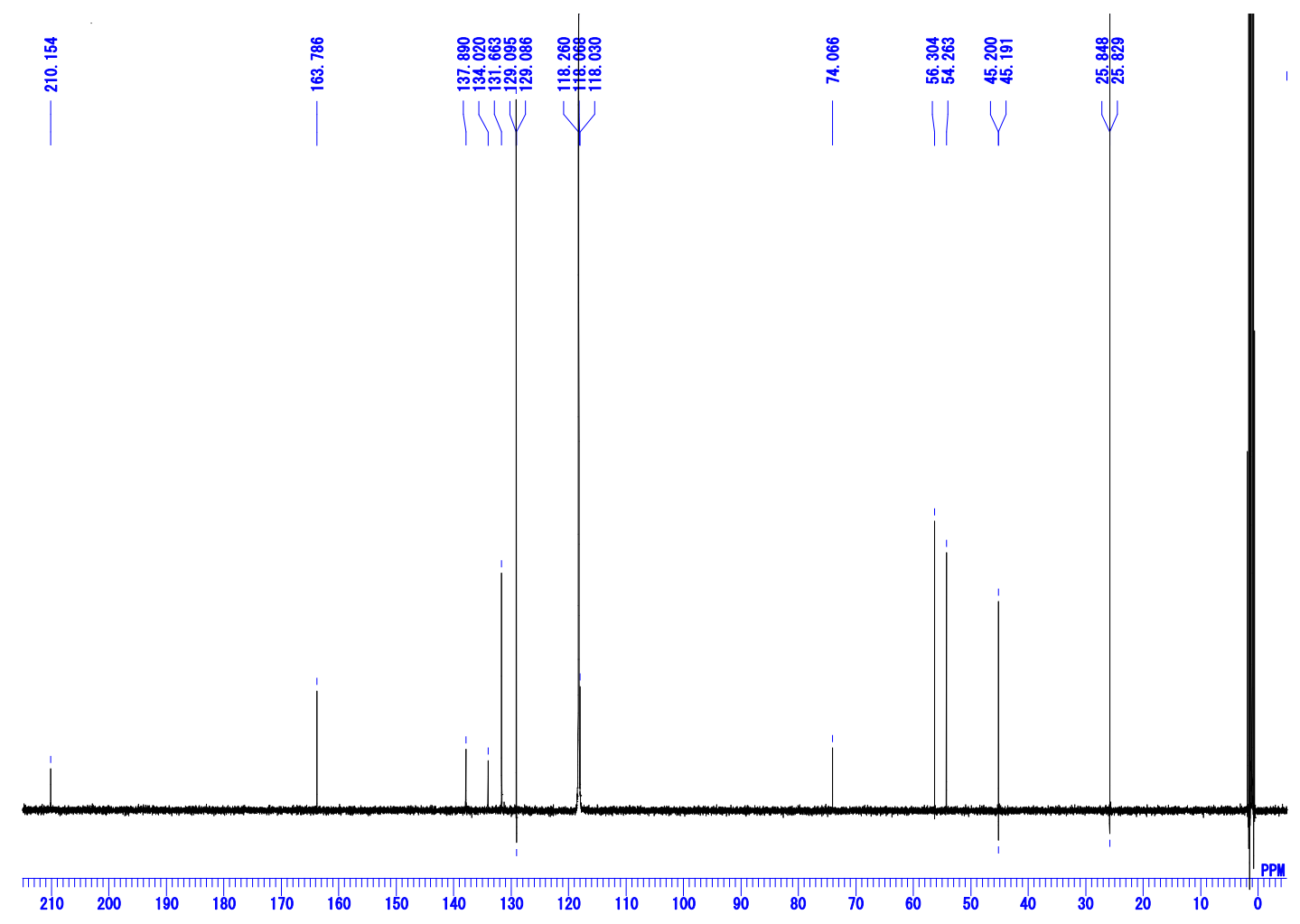

MW Profile

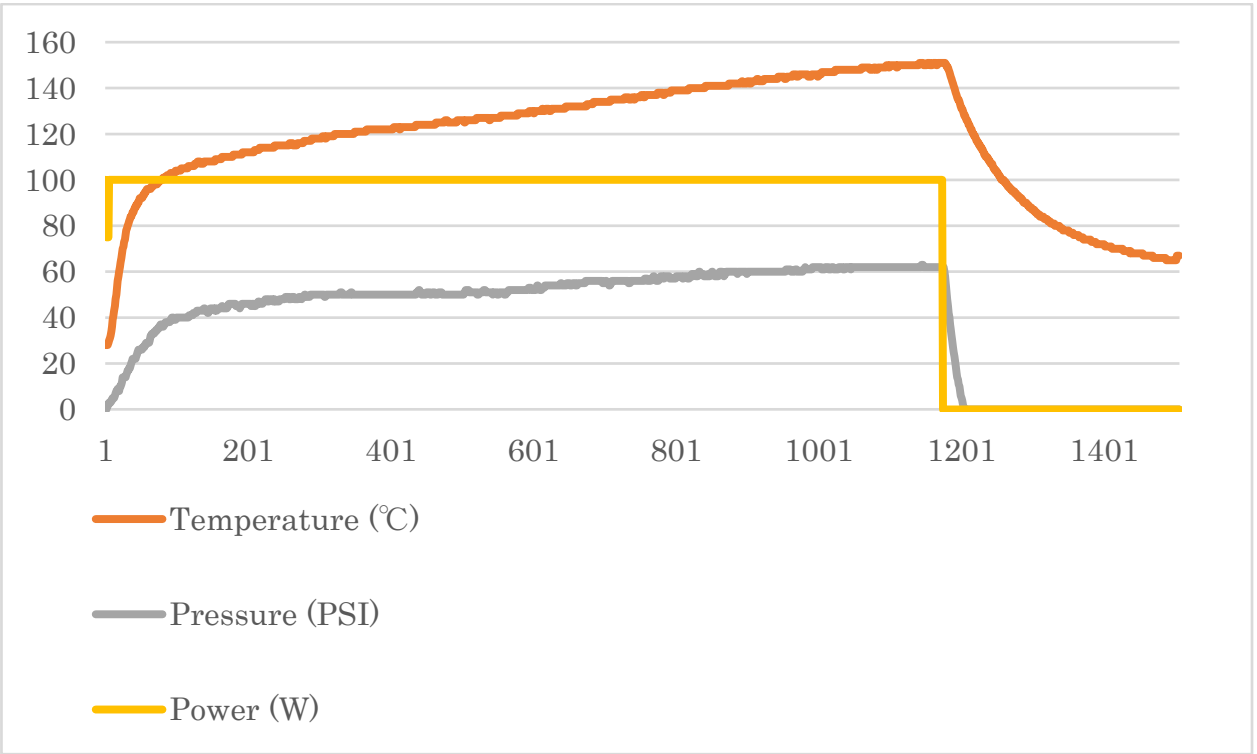


2-Amino-4-(4-chlorophenyl)-5-pivaloyl-4,5-dihydrothiophene-3-carbonitrile (cis-3bb)

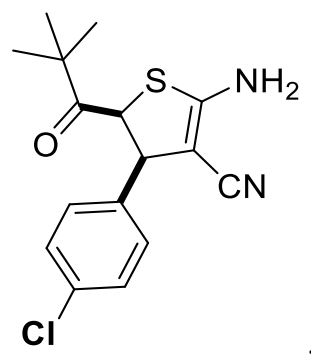

${ }^{1} \mathrm{H}$ NMR

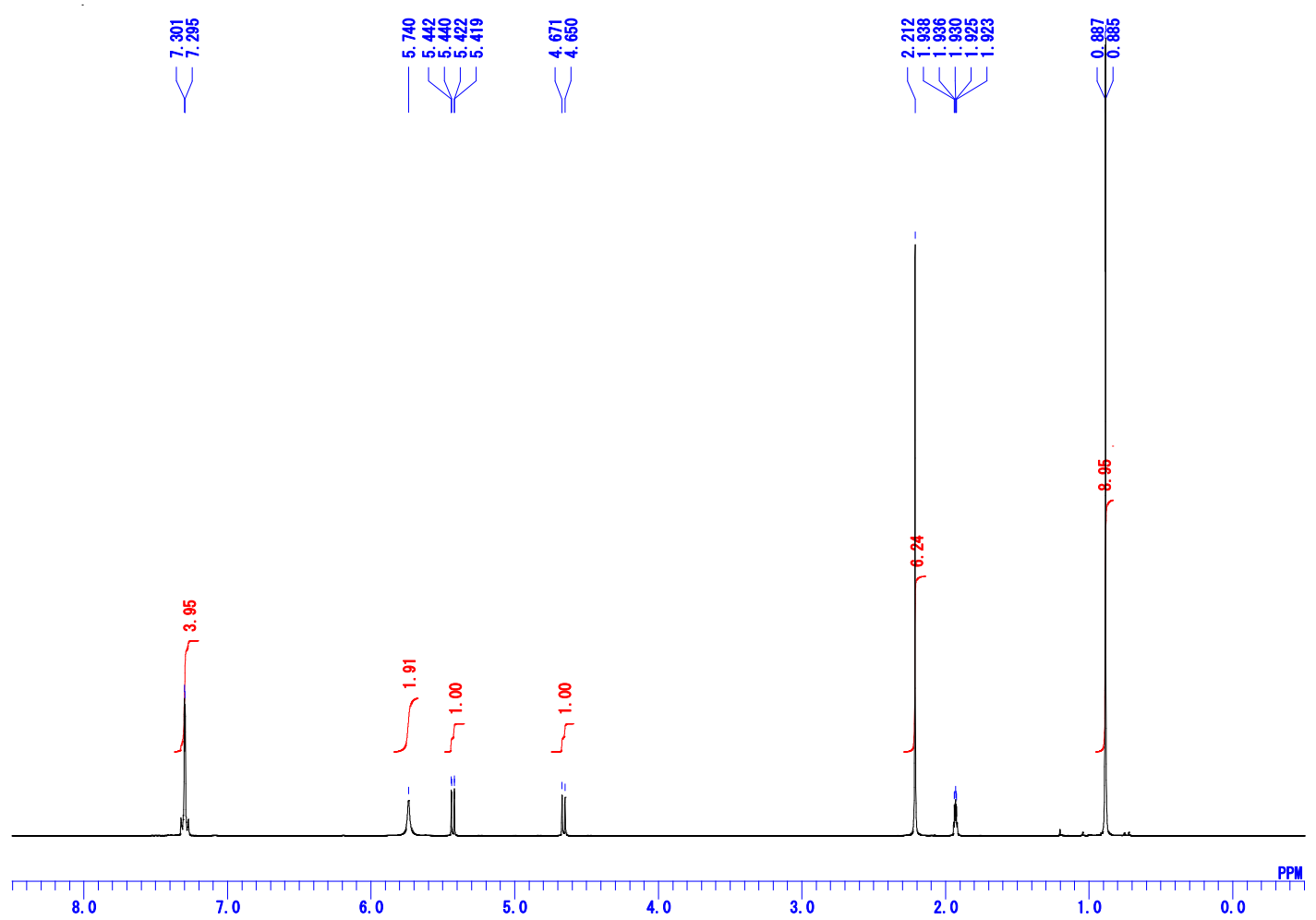


${ }^{13} \mathrm{C}$ NMR

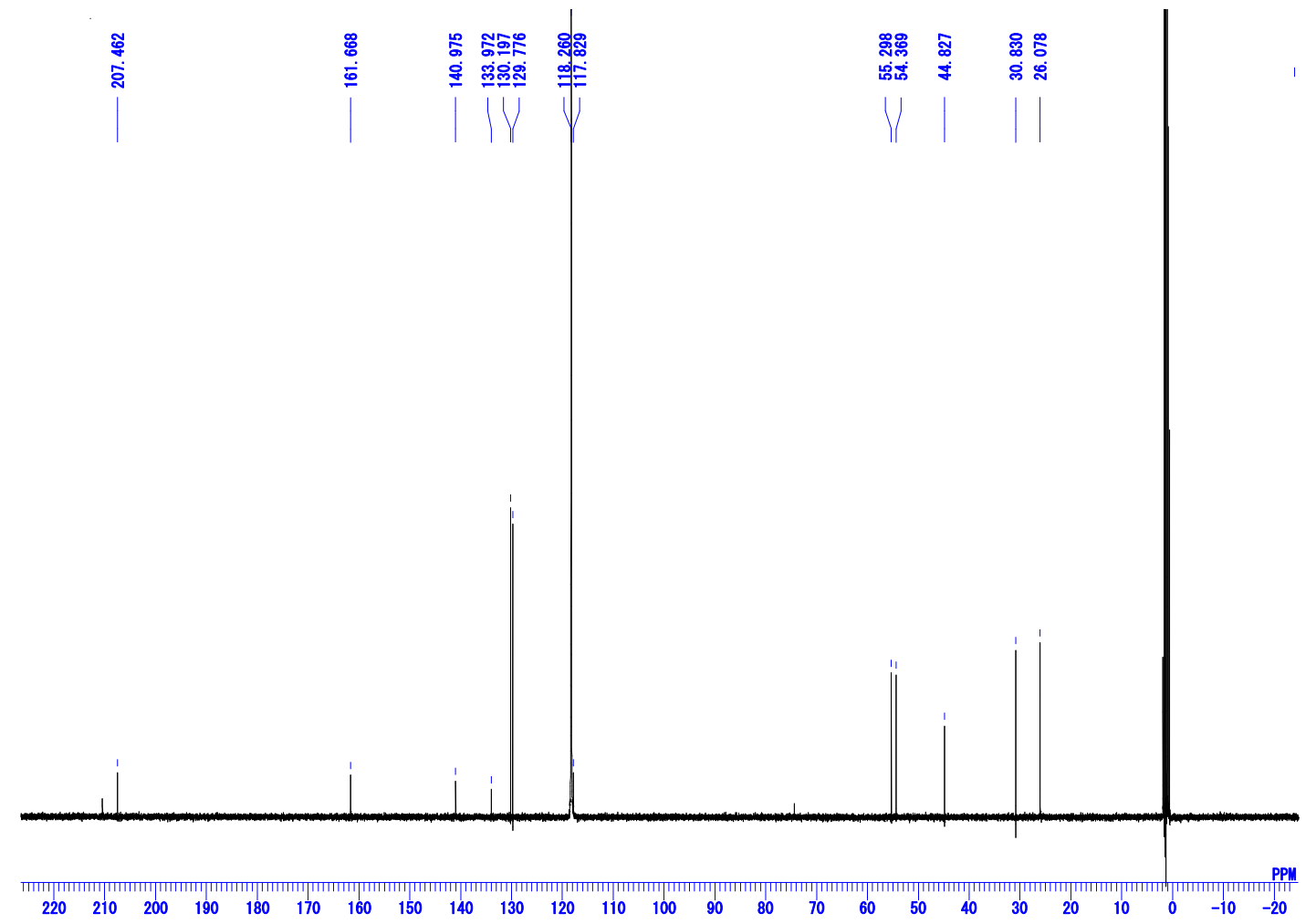

MW Profile

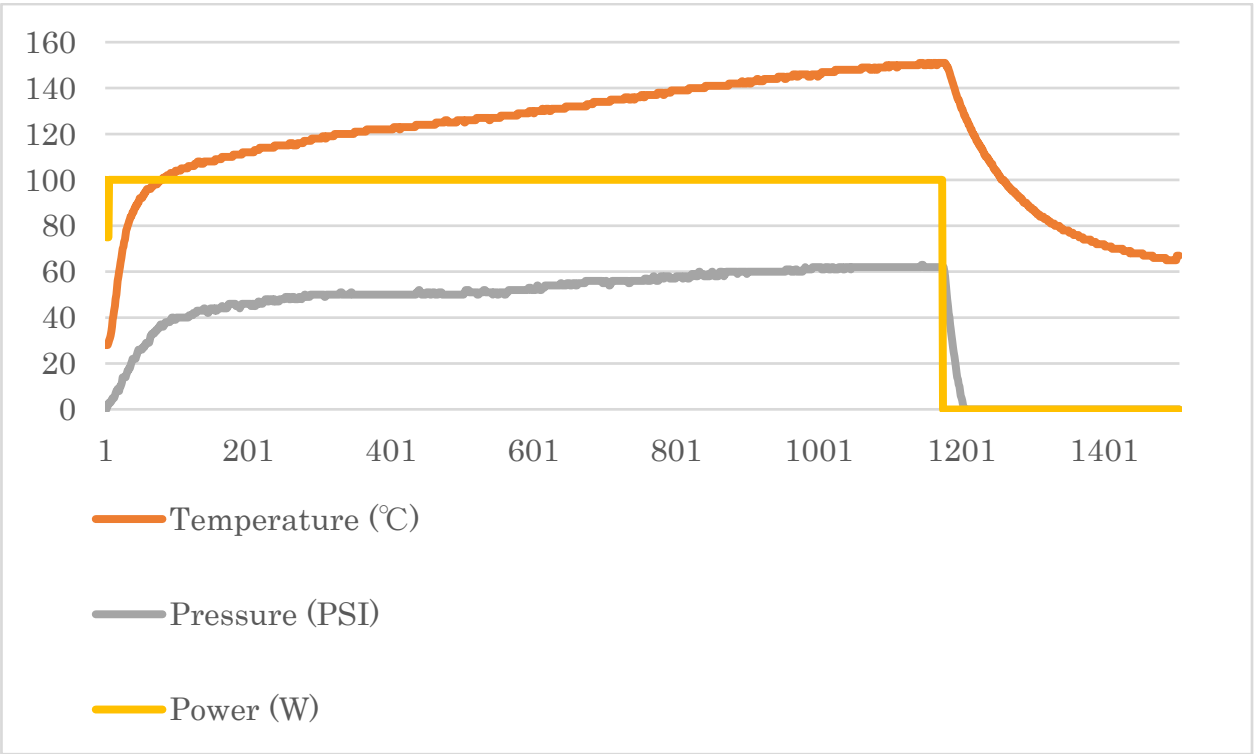


2-Amino-4-(4-bromophenyl)-5-pivaloyl-4,5-dihydrothiophene-3-carbonitrile (trans-3bh)

(CCDC; 195578) This was recrystallized in hexane and EtOAC. ORTEP drawing is at the 50\% probability level.
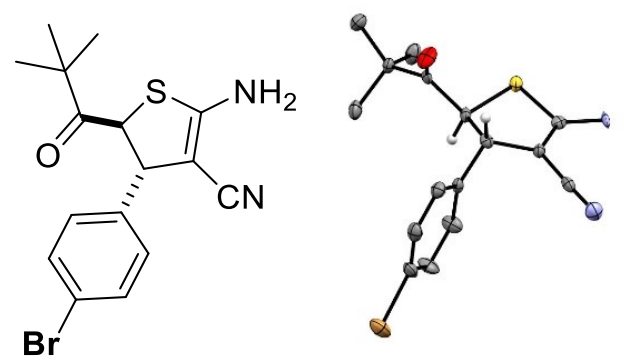

${ }^{1} \mathrm{H}$ NMR

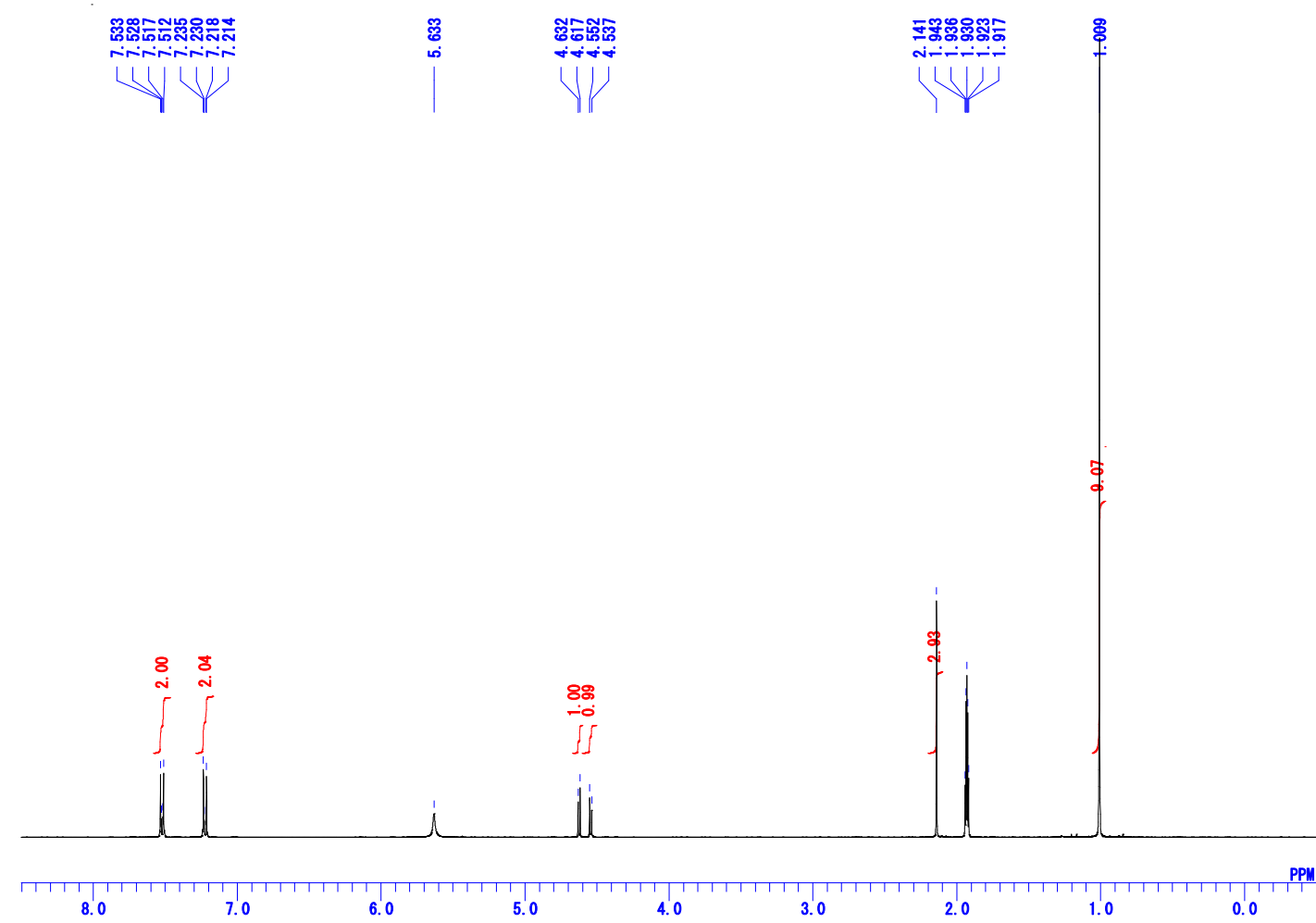


${ }^{13} \mathrm{C}$ NMR

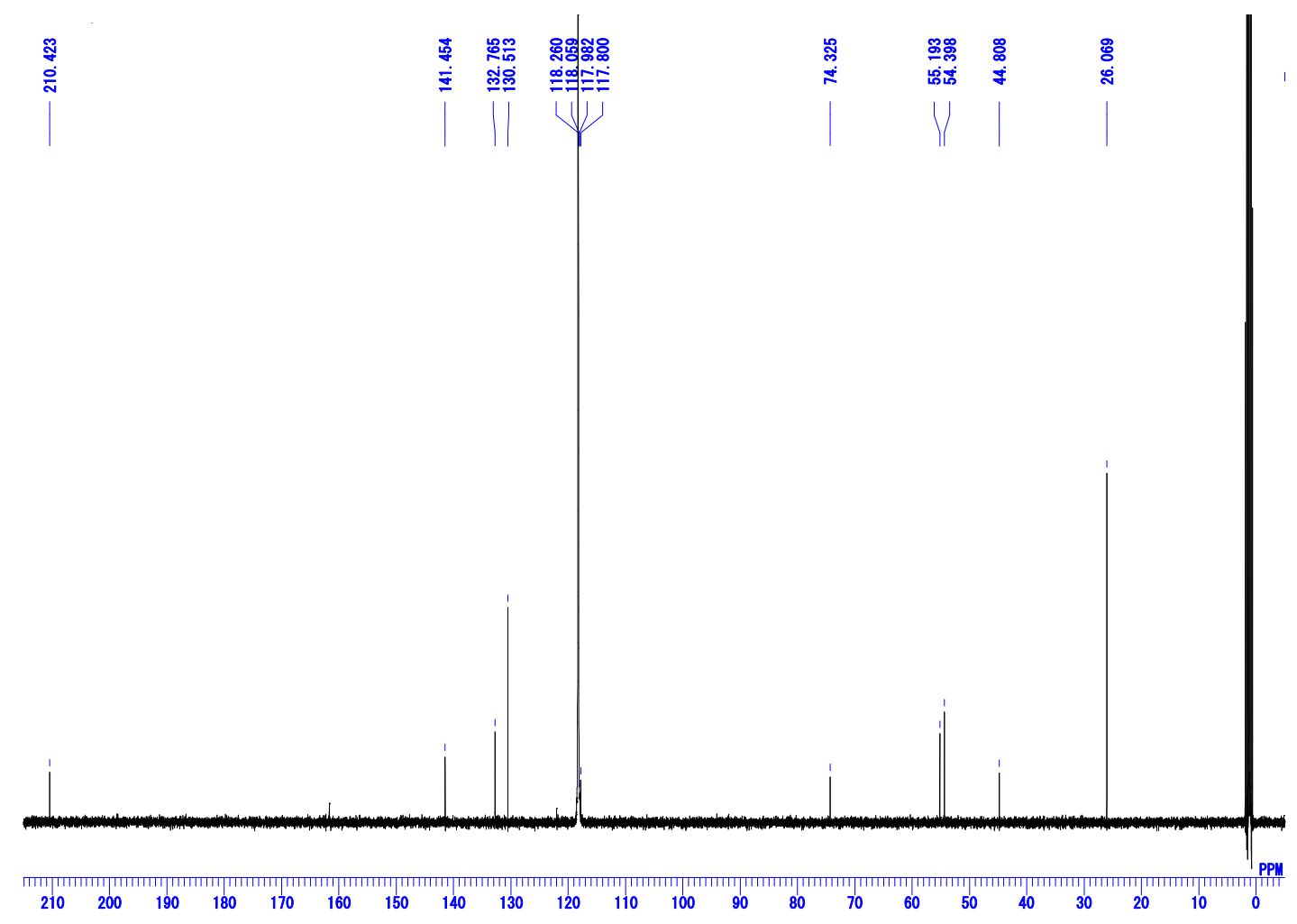

MW Profile

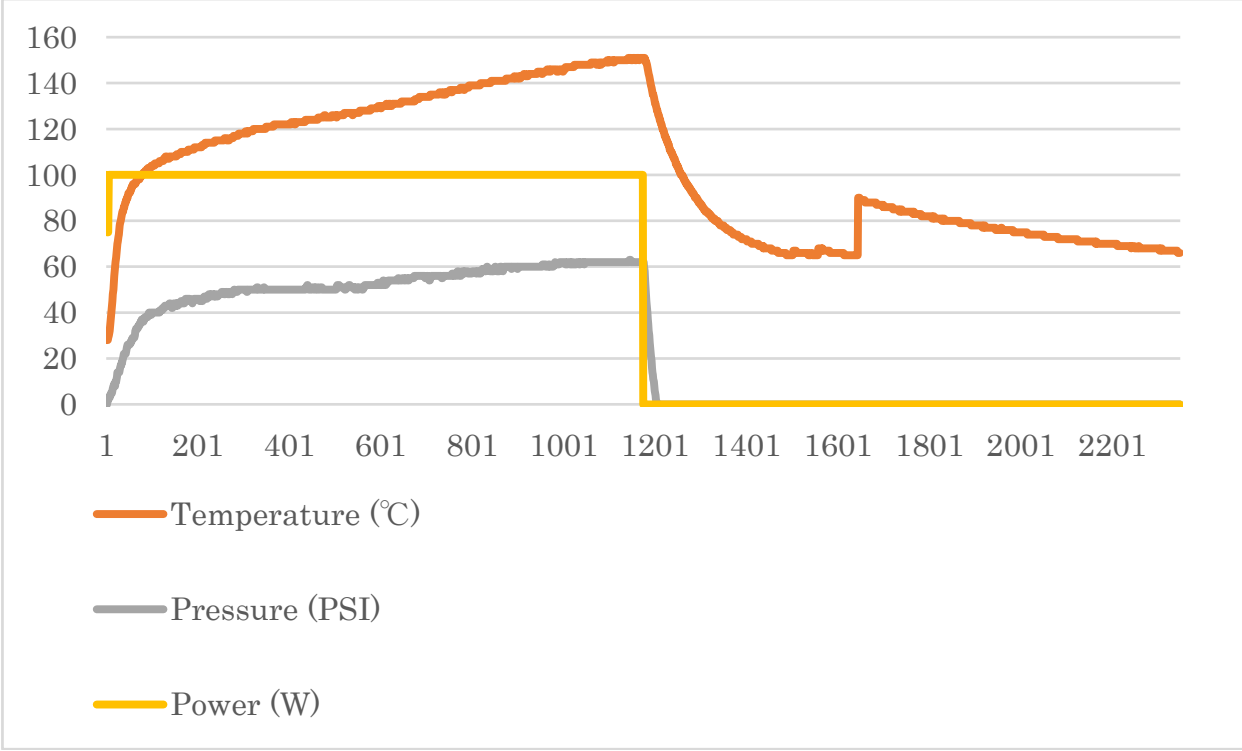


Crystal data and structure solution paramaters

\begin{tabular}{|c|c|c|c|}
\hline Empirical Formula & $\mathrm{C}_{16} \mathrm{H}_{17} \mathrm{BrN}_{2} \mathrm{OS}$ & Function Minimized & $\sum \mathrm{w}(|\mathrm{Fo}|-|\mathrm{Fc}|)^{2}$ \\
\hline Formula Weight & 365.29 & Least Squares Weights & $1 / \sigma^{2}(\mathrm{Fo})$ \\
\hline Crystal Color, Habit & colorless, prism & No. Observations $(\mathrm{I}>2.00 \sigma(\mathrm{I}))$ & 4757 \\
\hline Crystal Dimensions & $\begin{array}{l}0.100 \times 0.100 \times \\
0.100 \mathrm{~mm}\end{array}$ & No. Variables & 409 \\
\hline Crystal System & orthorhombic & Reflection/Parameter Ratio & 11.63 \\
\hline \multirow[t]{6}{*}{ Lattice Type } & Primitive & Residuals: R (I>2.00б(I)) & 0.0443 \\
\hline & $a=34.1256(6) \AA$ & 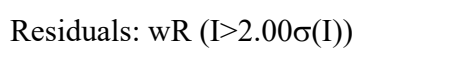 & 0.0384 \\
\hline & $\mathrm{b}=19.4576(4) \AA$ & Goodness of Fit Indicator & 2.390 \\
\hline & $\mathrm{c}=9.79393(18) \AA$ & Max Shift/Error in Final Cycle & 0.000 \\
\hline & $\mathrm{V}=6503.2(2) \AA^{3}$ & Maximum peak in Final Diff. Map & $0.84 \mathrm{e}^{-} / \AA^{3}$ \\
\hline & & Minimum peak in Final Diff. Map & $-0.88 \mathrm{e}^{-} / \AA^{3}$ \\
\hline Space Group & $\operatorname{Pccn}(\# 56)$ & & \\
\hline$Z$ value & 16 & & \\
\hline$D_{\text {cal }}$ & $1.492 \mathrm{~g} / \mathrm{cm}^{3}$ & & \\
\hline$F_{000}$ & 2976.00 & & \\
\hline$\mu(\mathrm{CuK} \alpha)$ & $46.442 \mathrm{~cm}^{-1}$ & & \\
\hline Temperature & & & \\
\hline
\end{tabular}


2-Amino-4-(4-bromophenyl)-5-pivaloyl-4,5-dihydrothiophene-3-carbonitrile (cis-3bh)

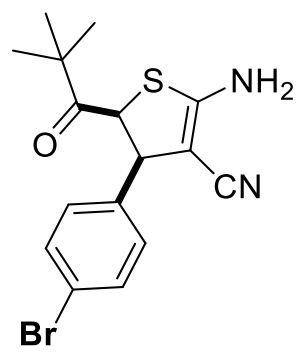

${ }^{1} \mathrm{H}$ NMR

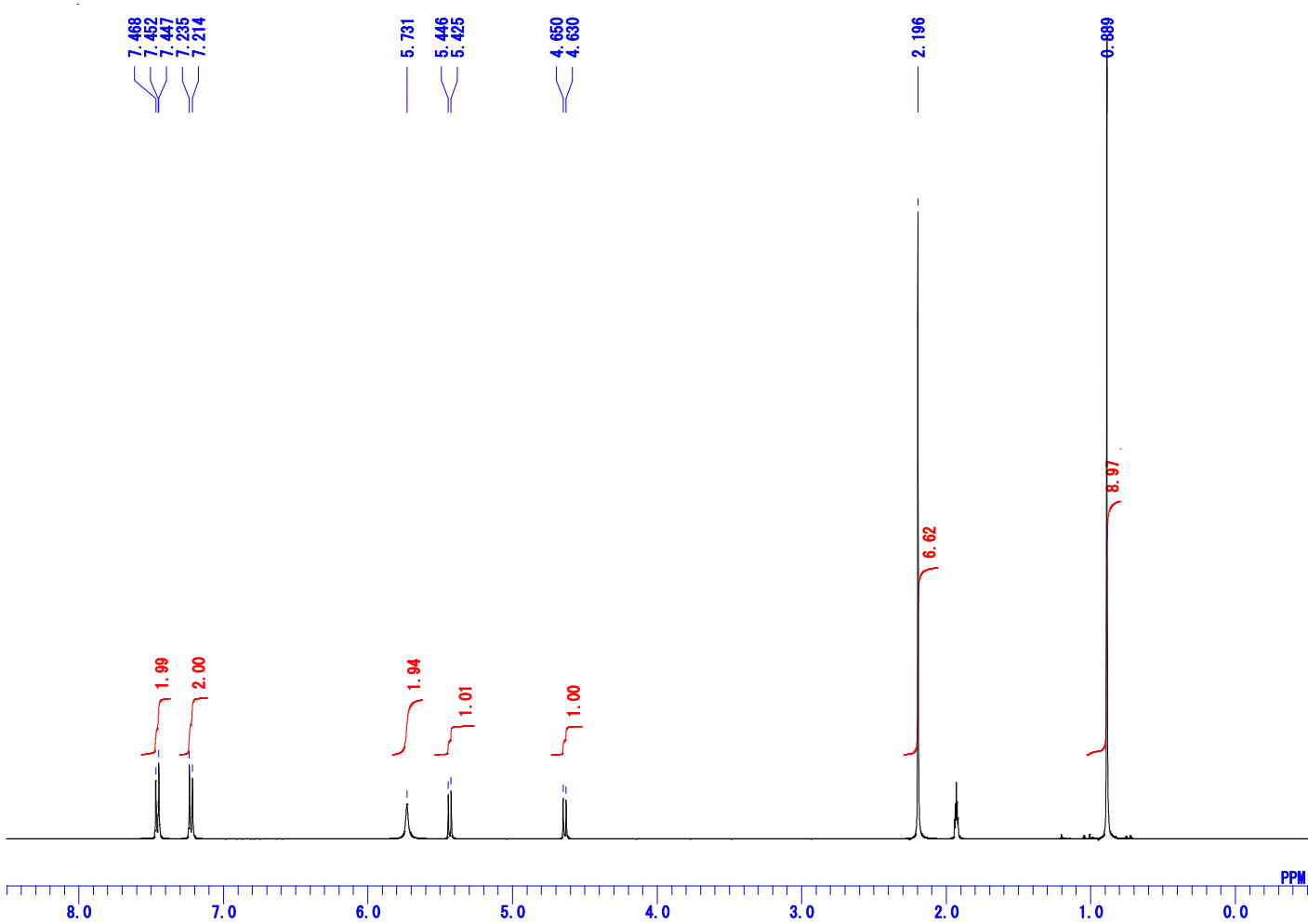


${ }^{13} \mathrm{C}$ NMR

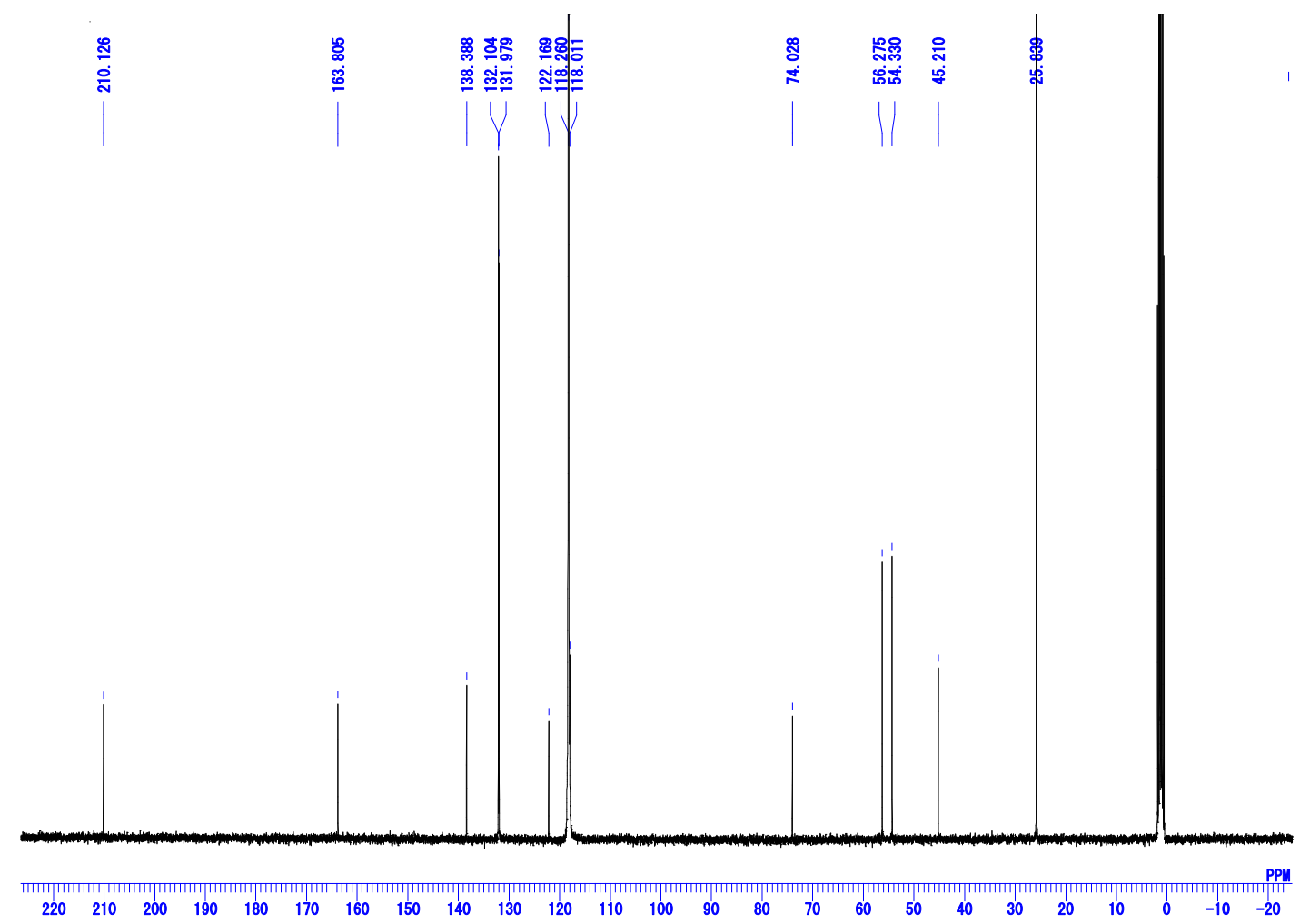

MW Profile

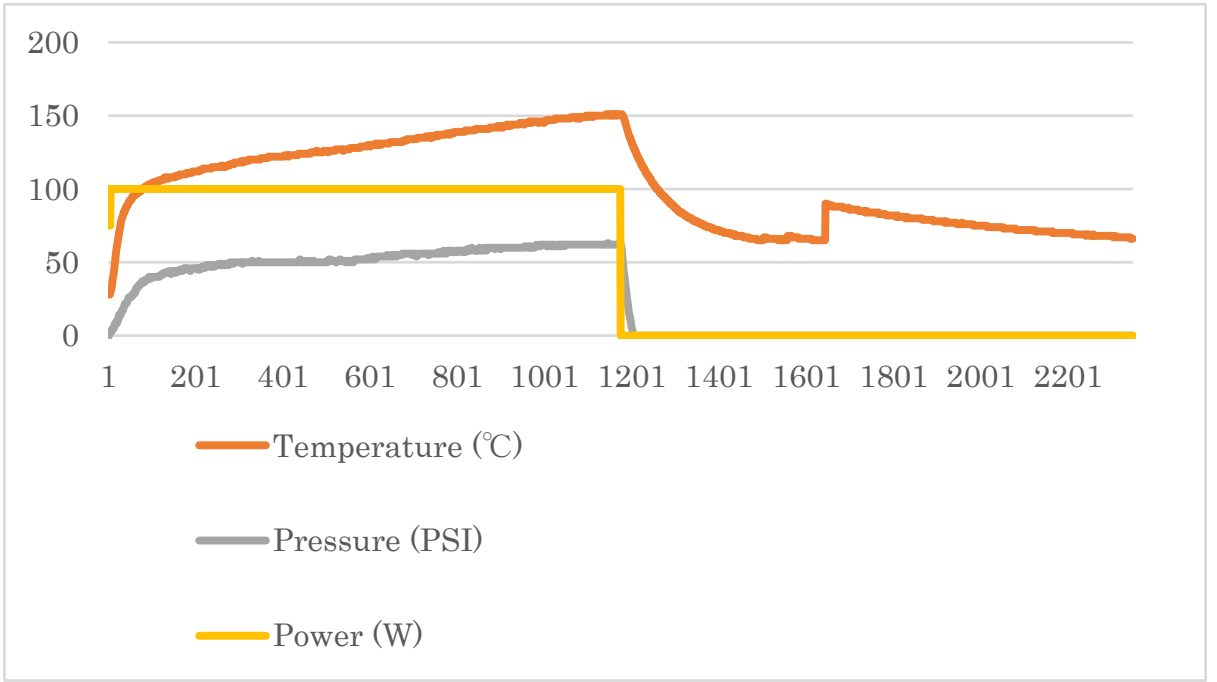


2-Amino-5-pivaloyl-4-(p-tolyl)-4,5-dihydrothiophene-3-carbonitrile (trans-3bc)

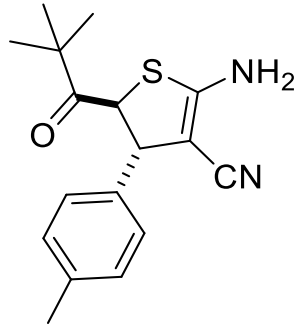

${ }^{1} \mathrm{H}$ NMR

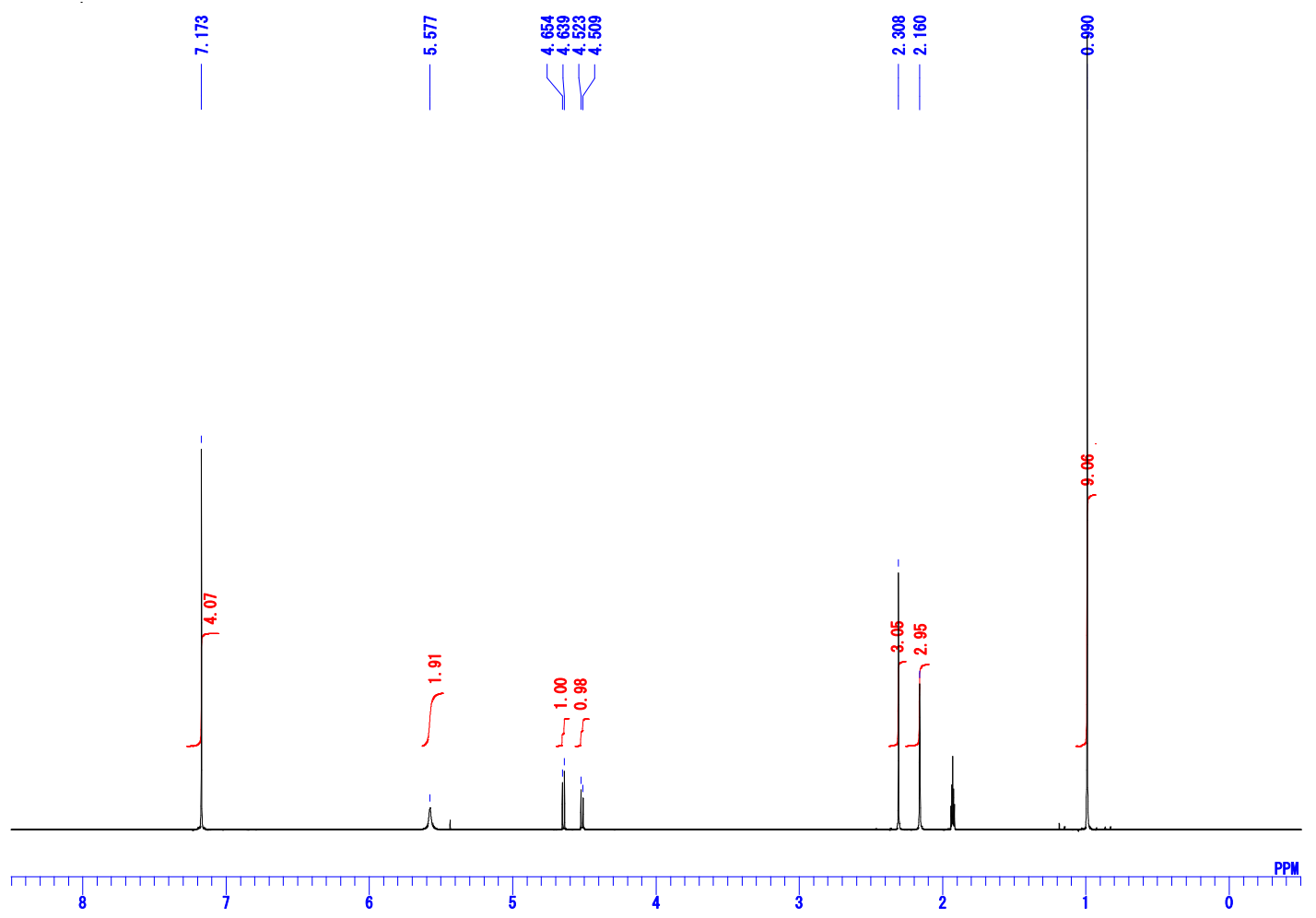


${ }^{13} \mathrm{C}$ NMR

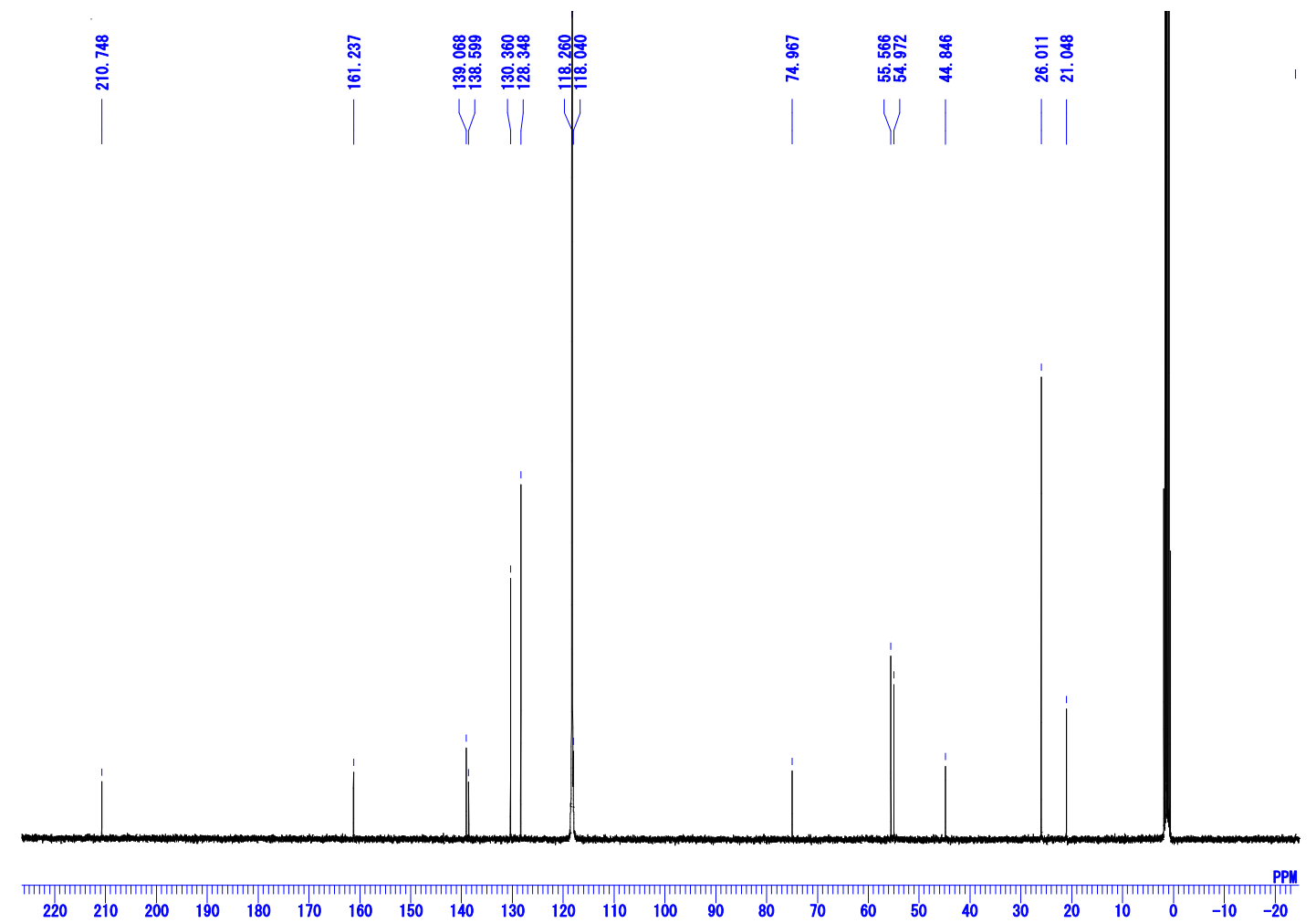

MW Profile

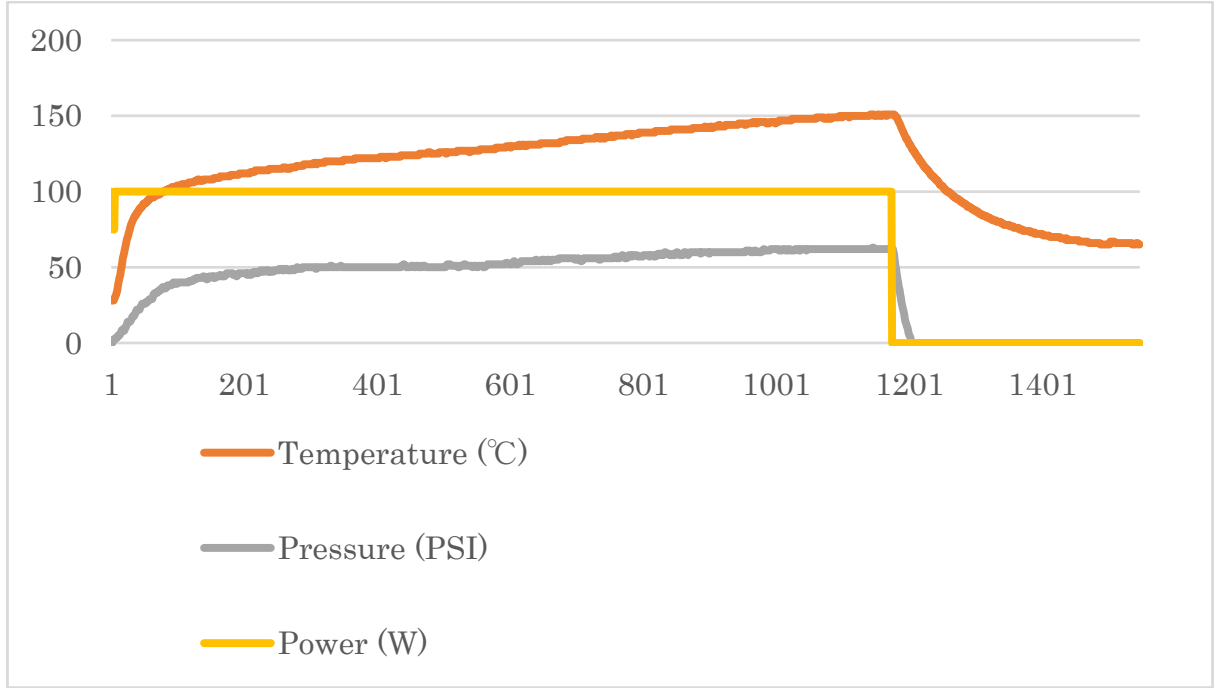


2-Amino-5-pivaloyl-4-(p-tolyl)-4,5-dihydrothiophene-3-carbonitrile (cis-3bc)

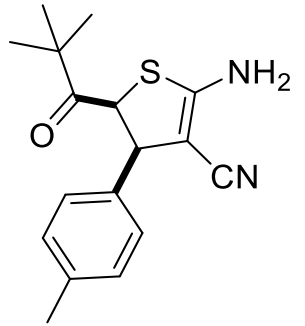

${ }^{1} \mathrm{H}$ NMR

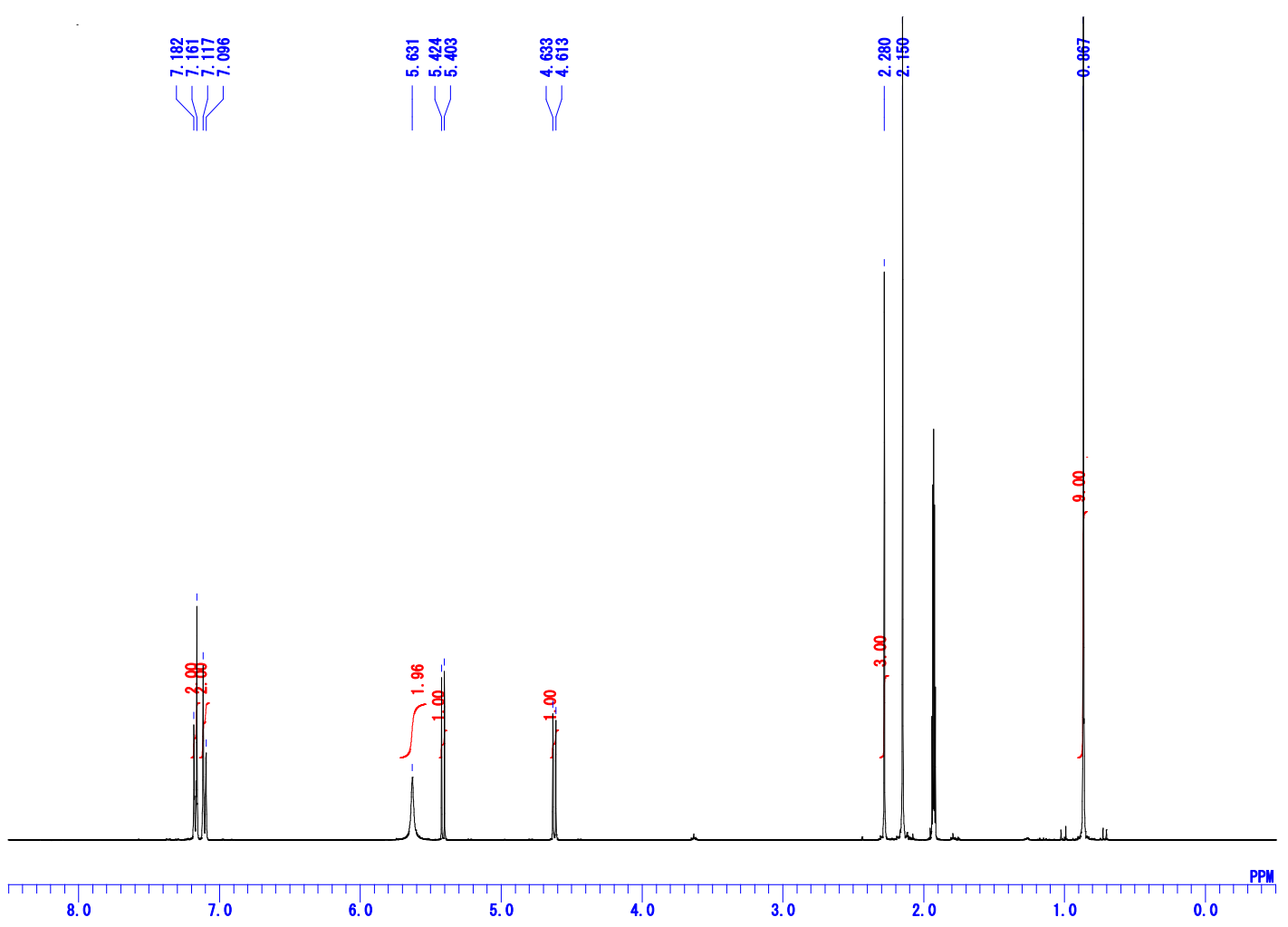


${ }^{13} \mathrm{C}$ NMR

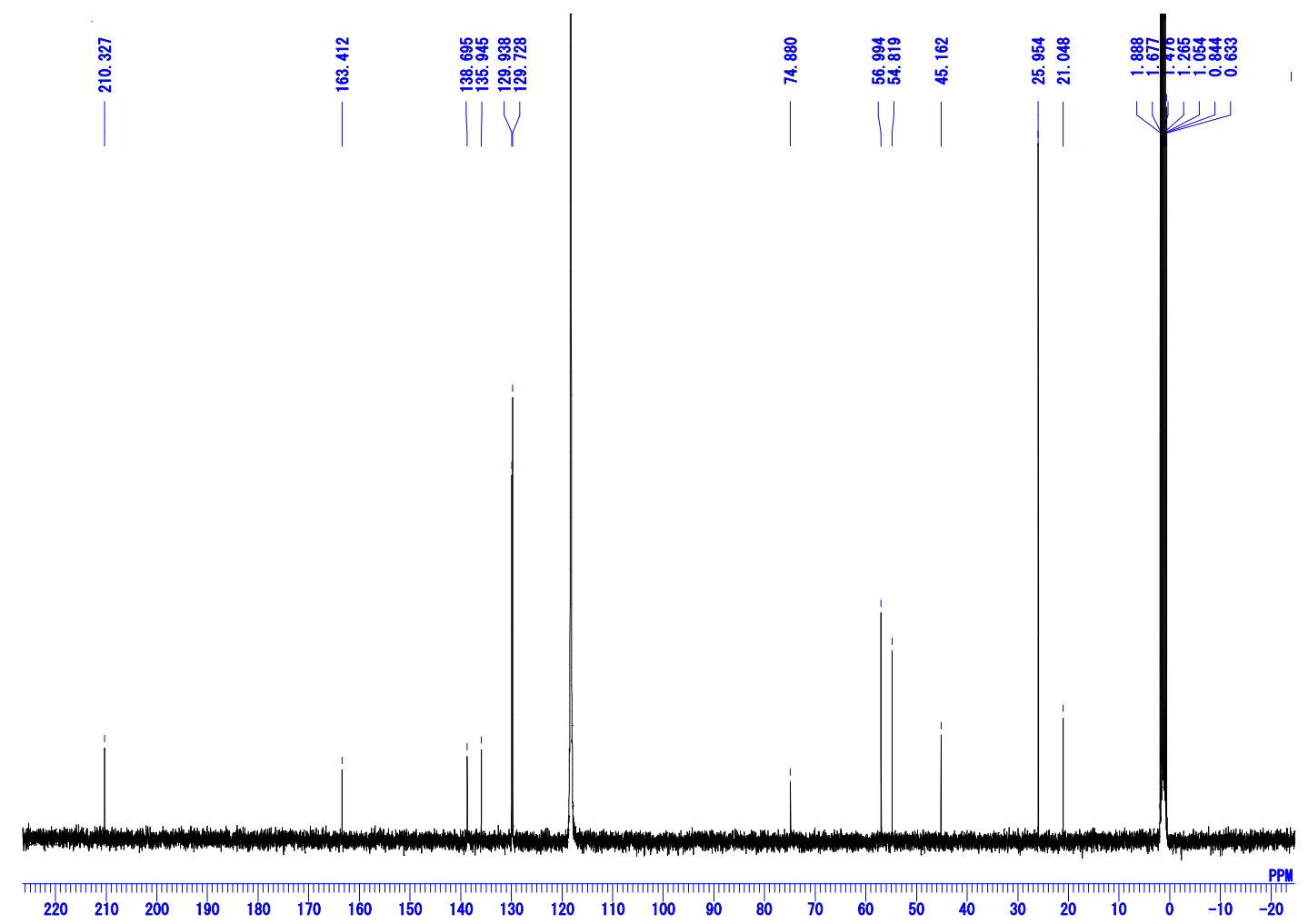

MW Profile

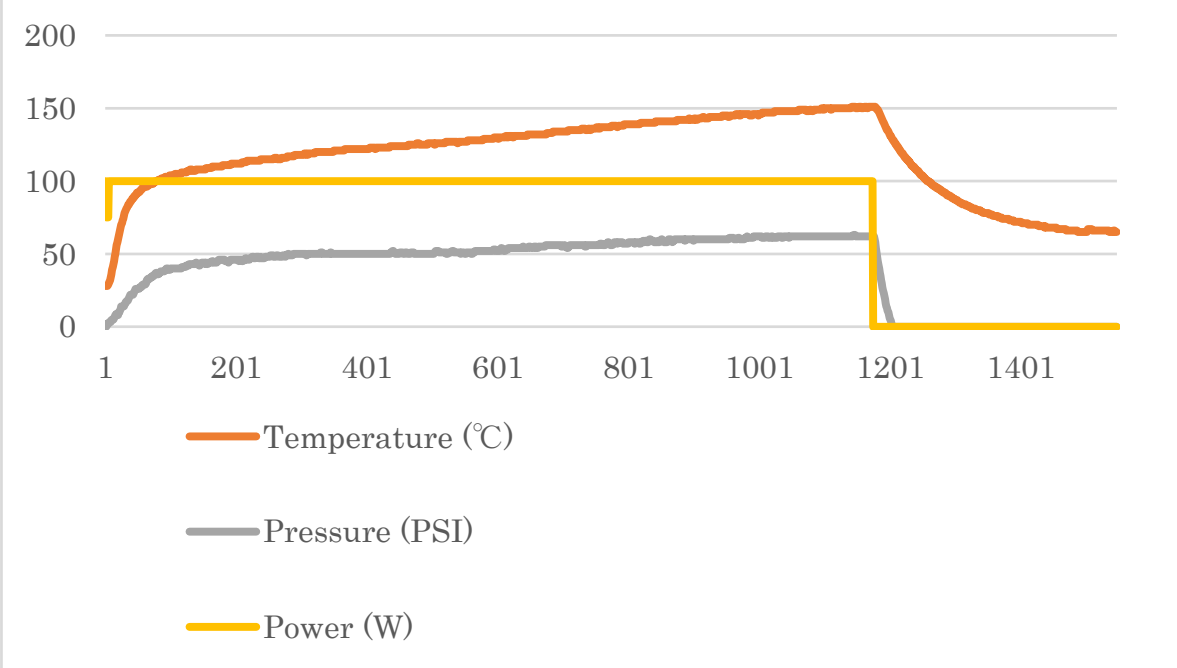


2-Amino-4-(4-methoxyphenyl)-5-pivaloyl-4,5-dihydrothiophene-3-carbonitrile (trans-3bd)

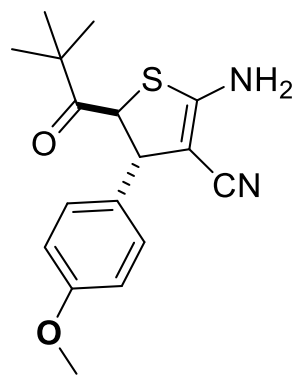

${ }^{1} \mathrm{H}$ NMR

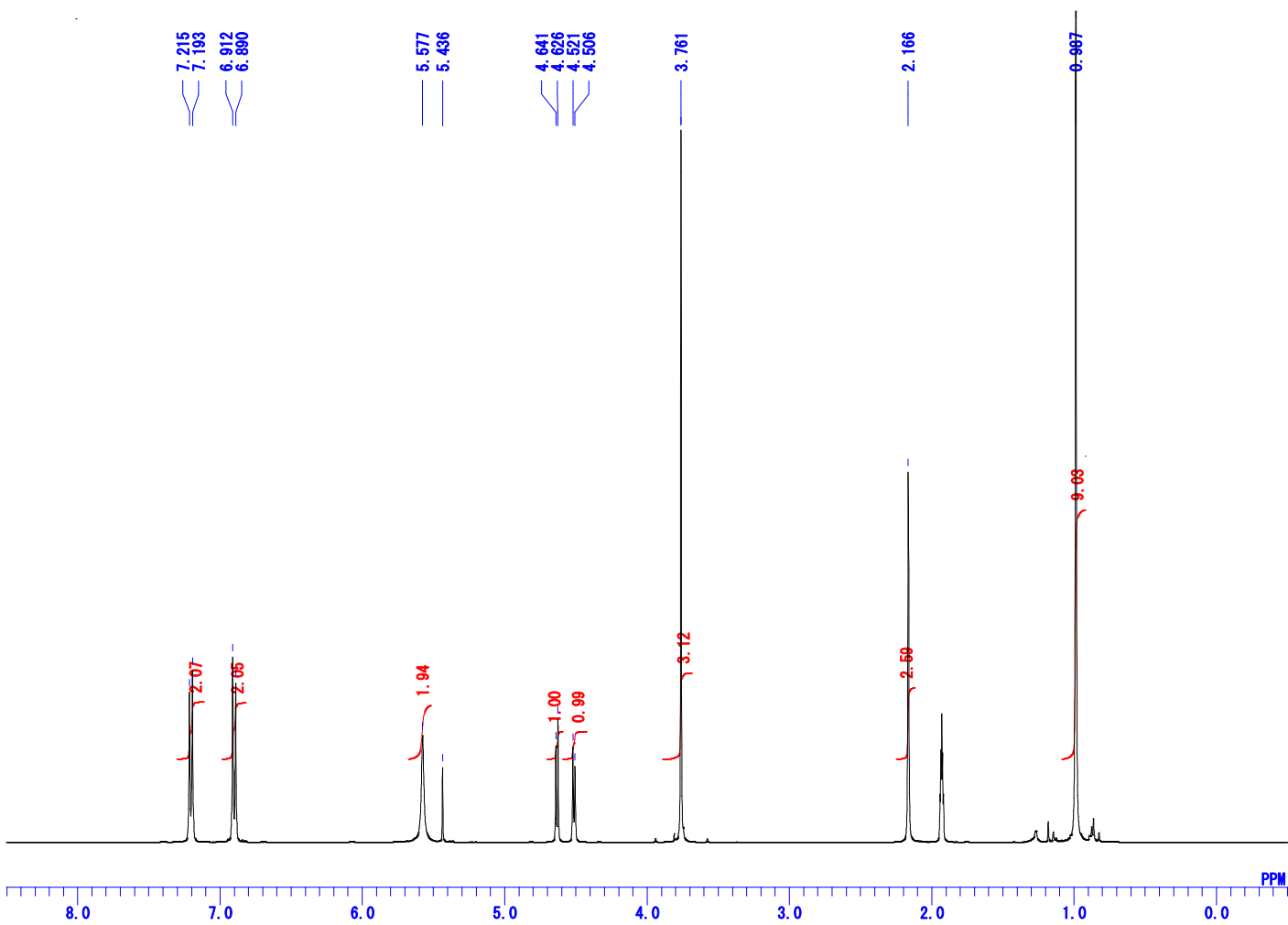


${ }^{13} \mathrm{C}$ NMR

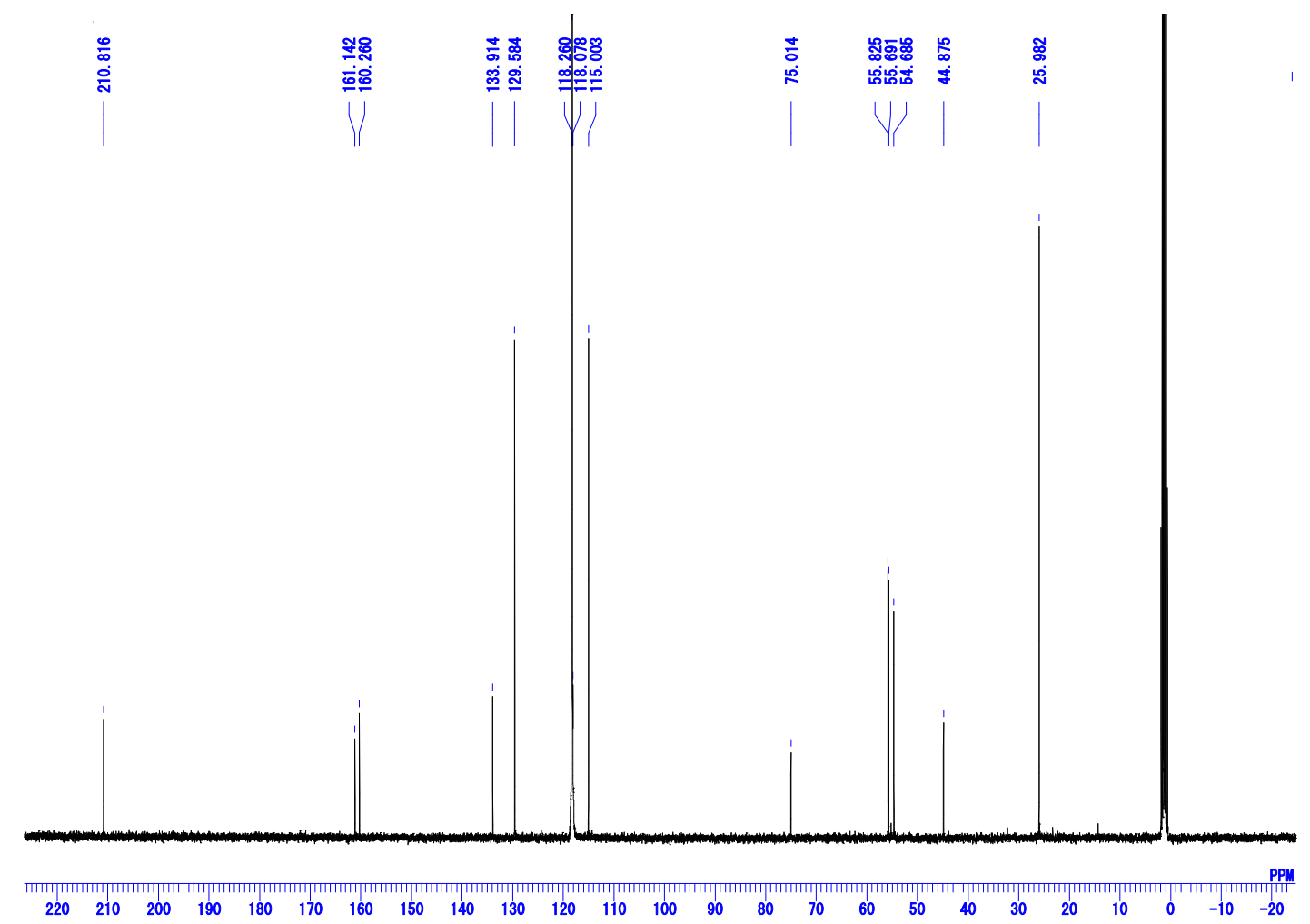

MW Profile

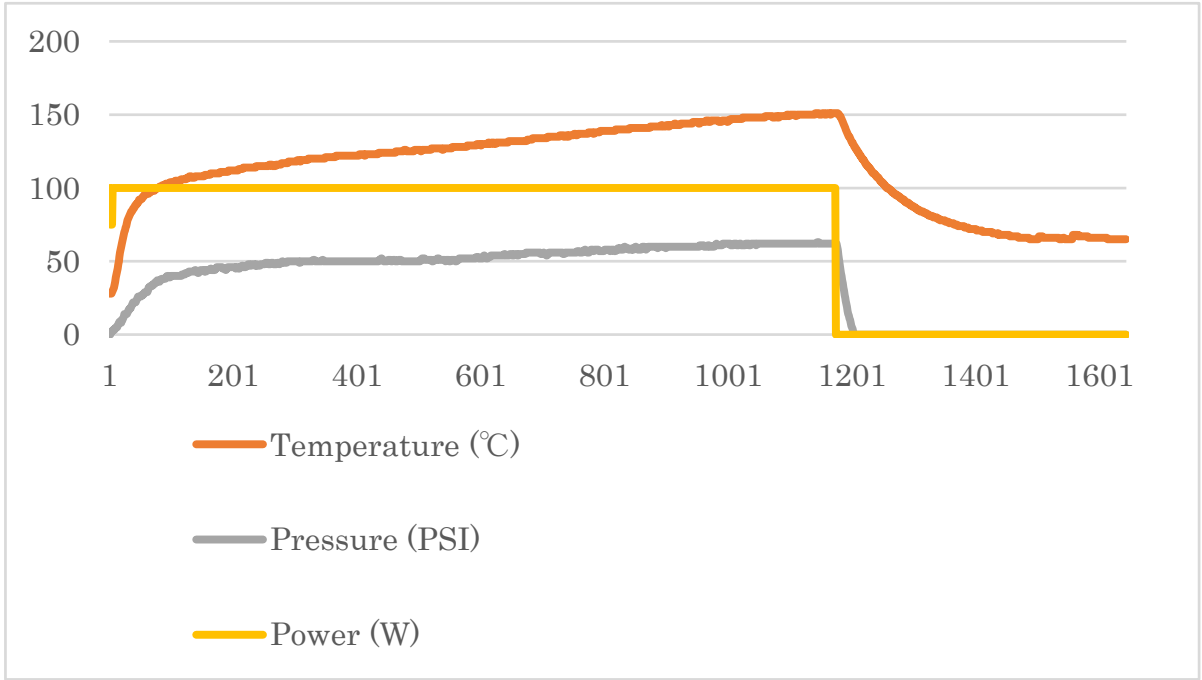


2-Amino-4-(4-methoxyphenyl)-5-pivaloyl-4,5-dihydrothiophene-3-carbonitrile (cis-3bd)

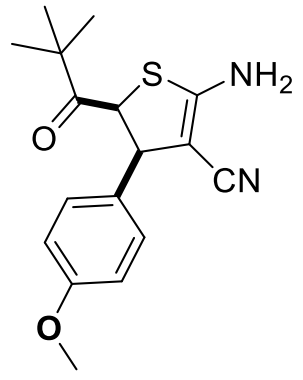

${ }^{1} \mathrm{H}$ NMR

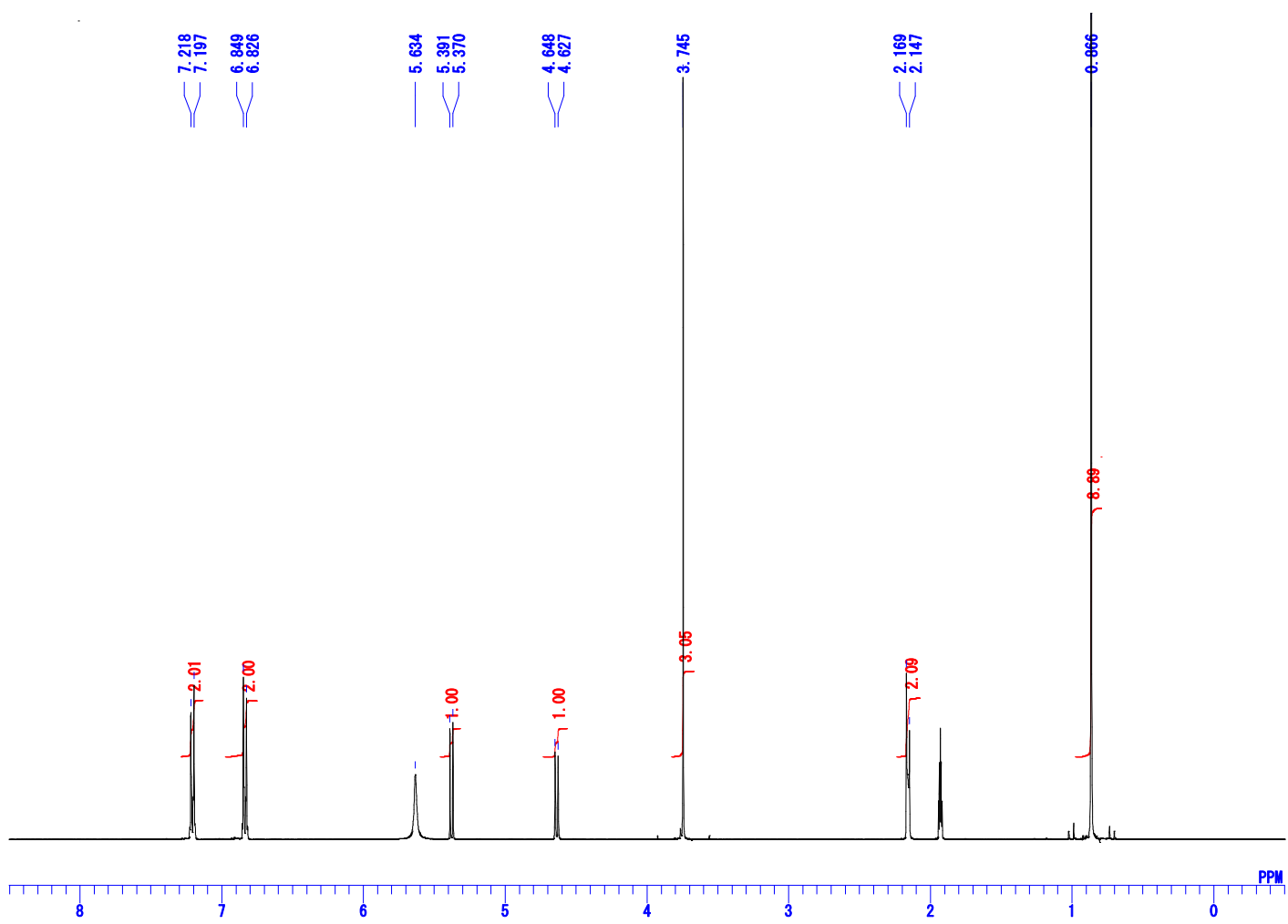


${ }^{13} \mathrm{C}$ NMR

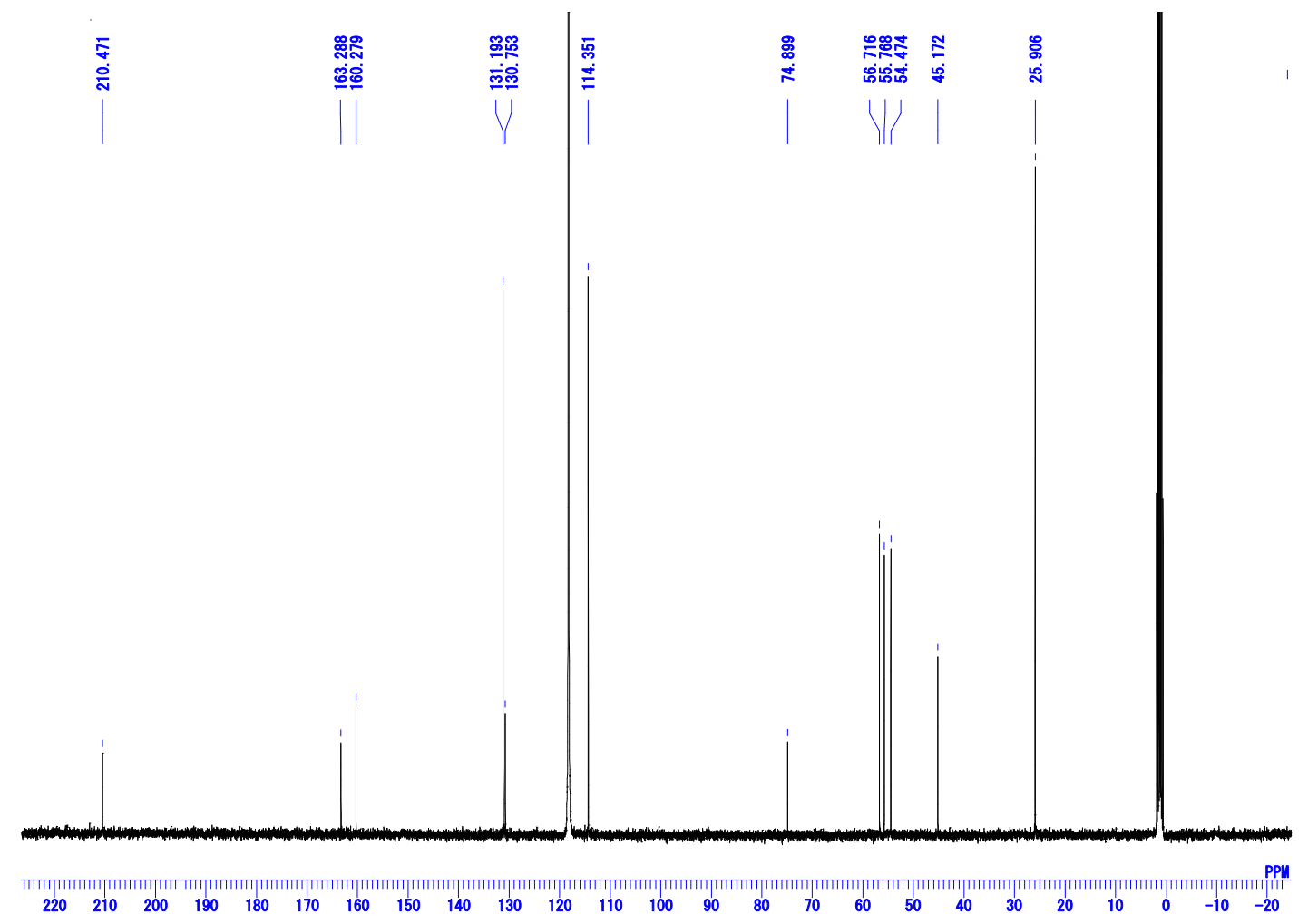

MW Profile

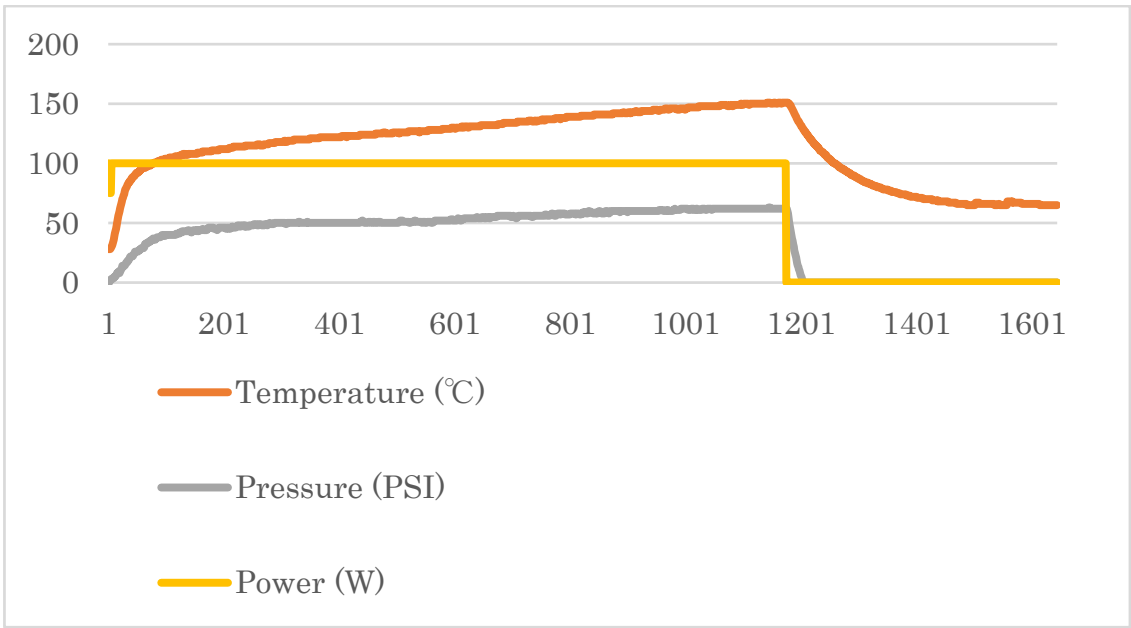


2-Amino-4-(naphthalen-2-yl)-5-pivaloyl-4,5-dihydrothiophene-3-carbonitrile (trans-3be)

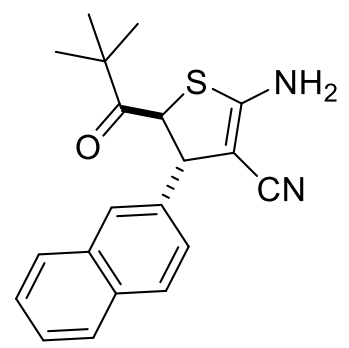

${ }^{1} \mathrm{H}$ NMR

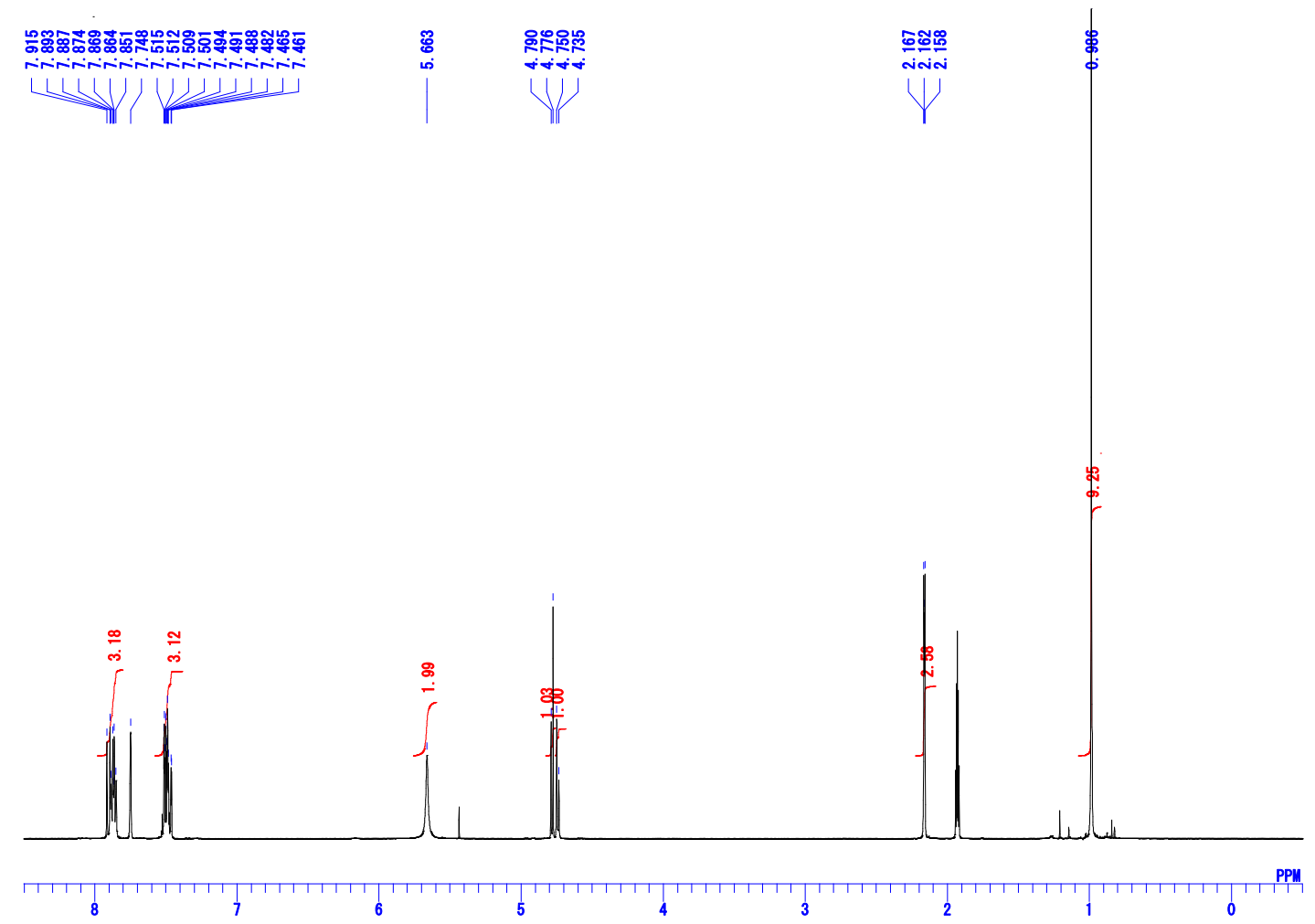


${ }^{13} \mathrm{C}$ NMR

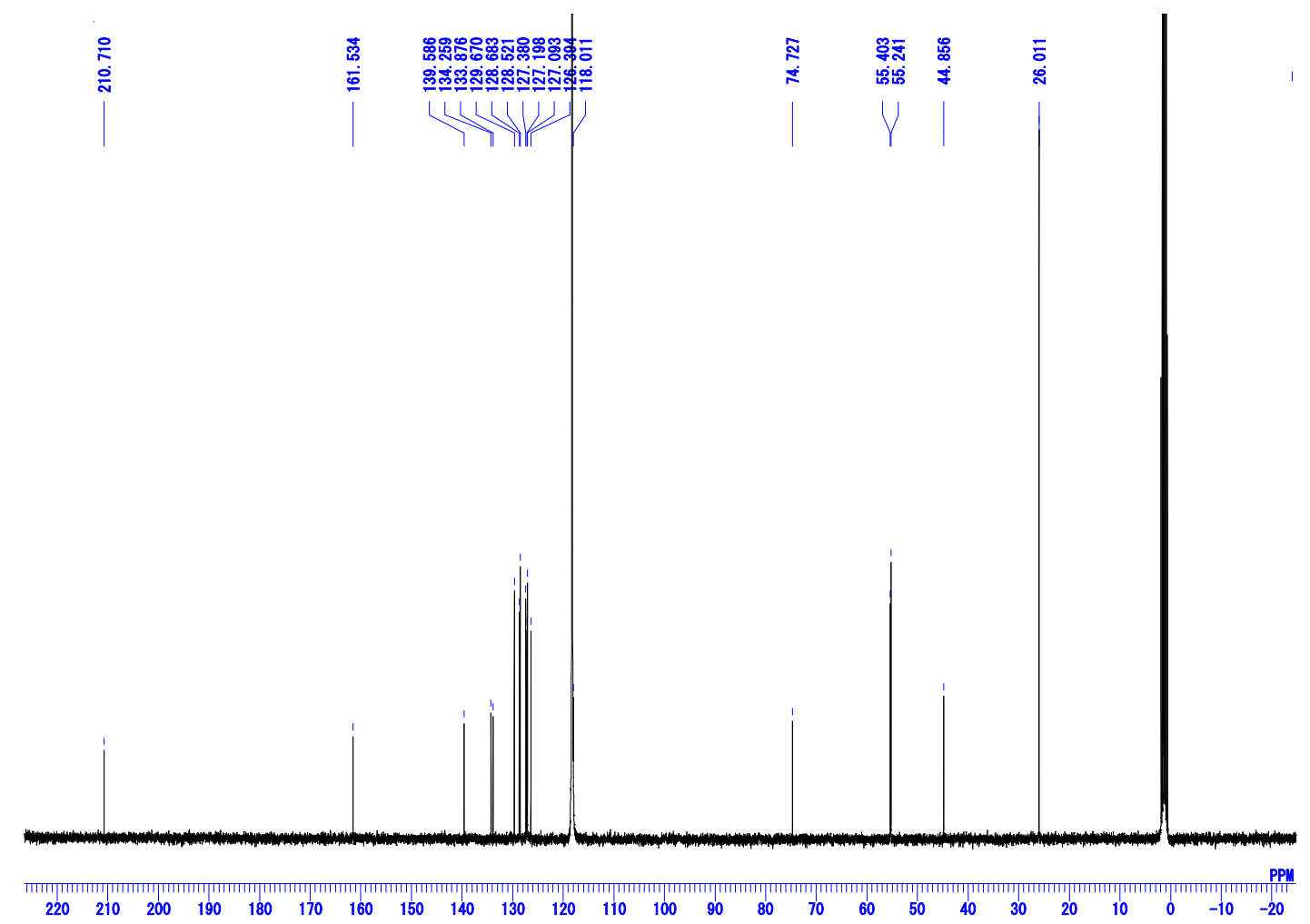

MW Profile

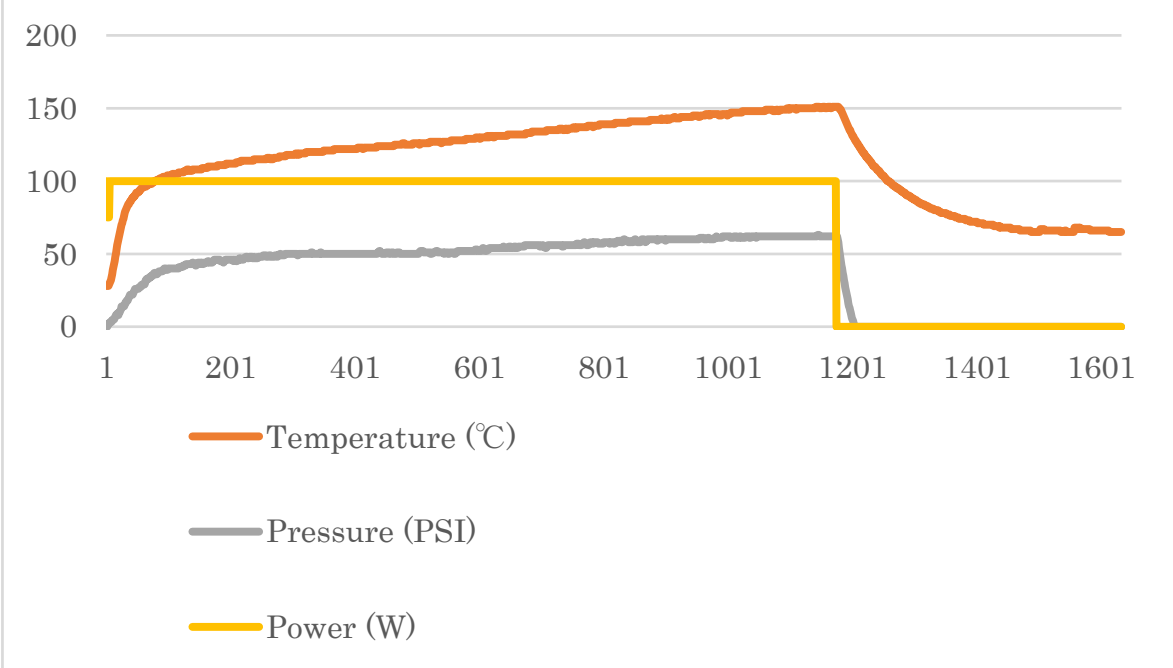


2-Amino-4-(naphthalen-2-yl)-5-pivaloyl-4,5-dihydrothiophene-3-carbonitrile (cis-3be)

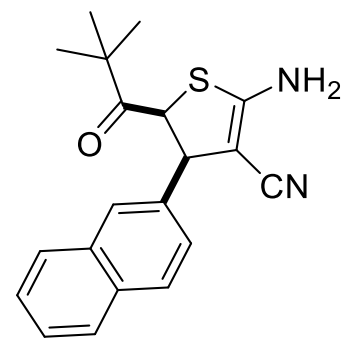

${ }^{1} \mathrm{H}$ NMR

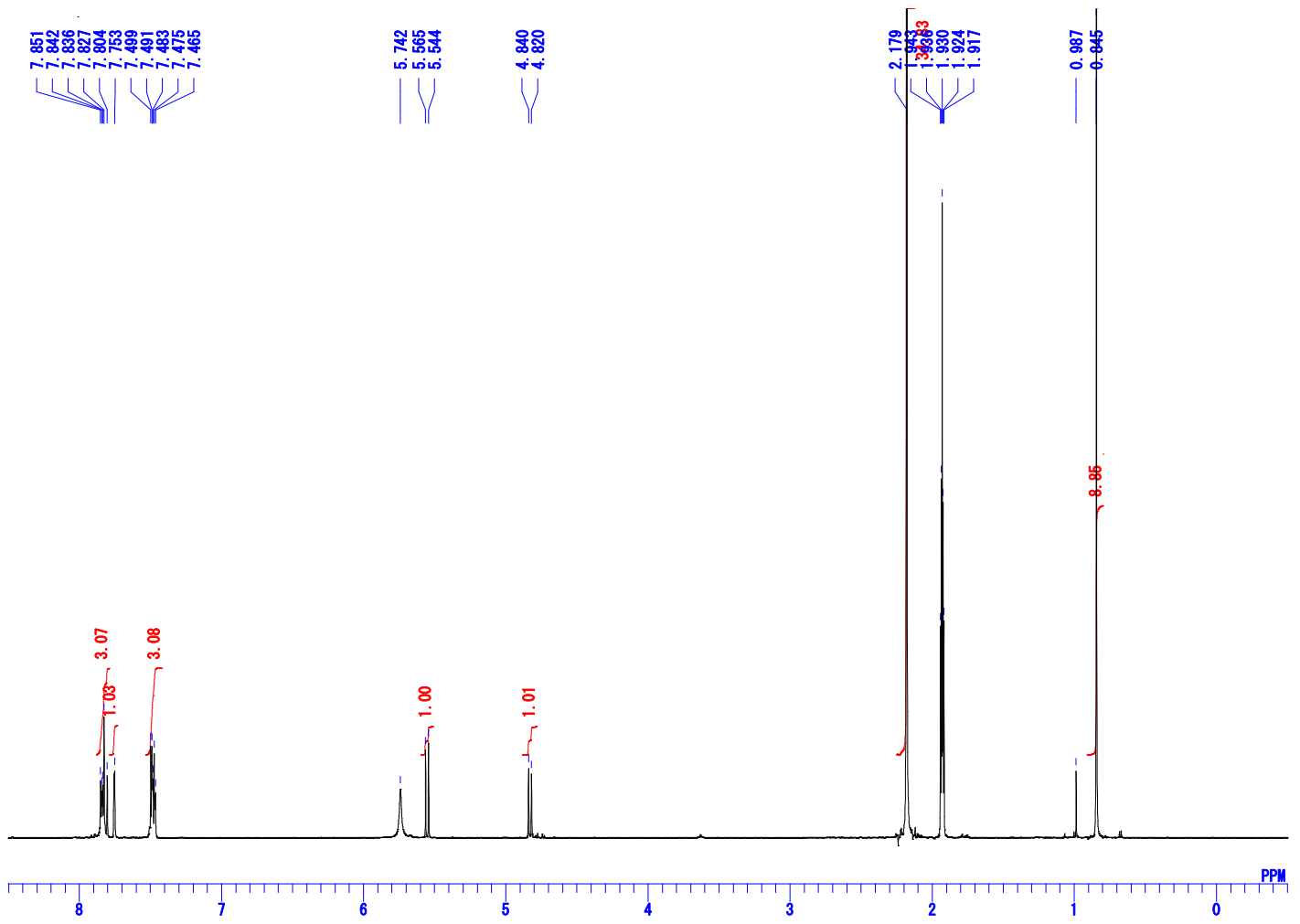


${ }^{13} \mathrm{C}$ NMR

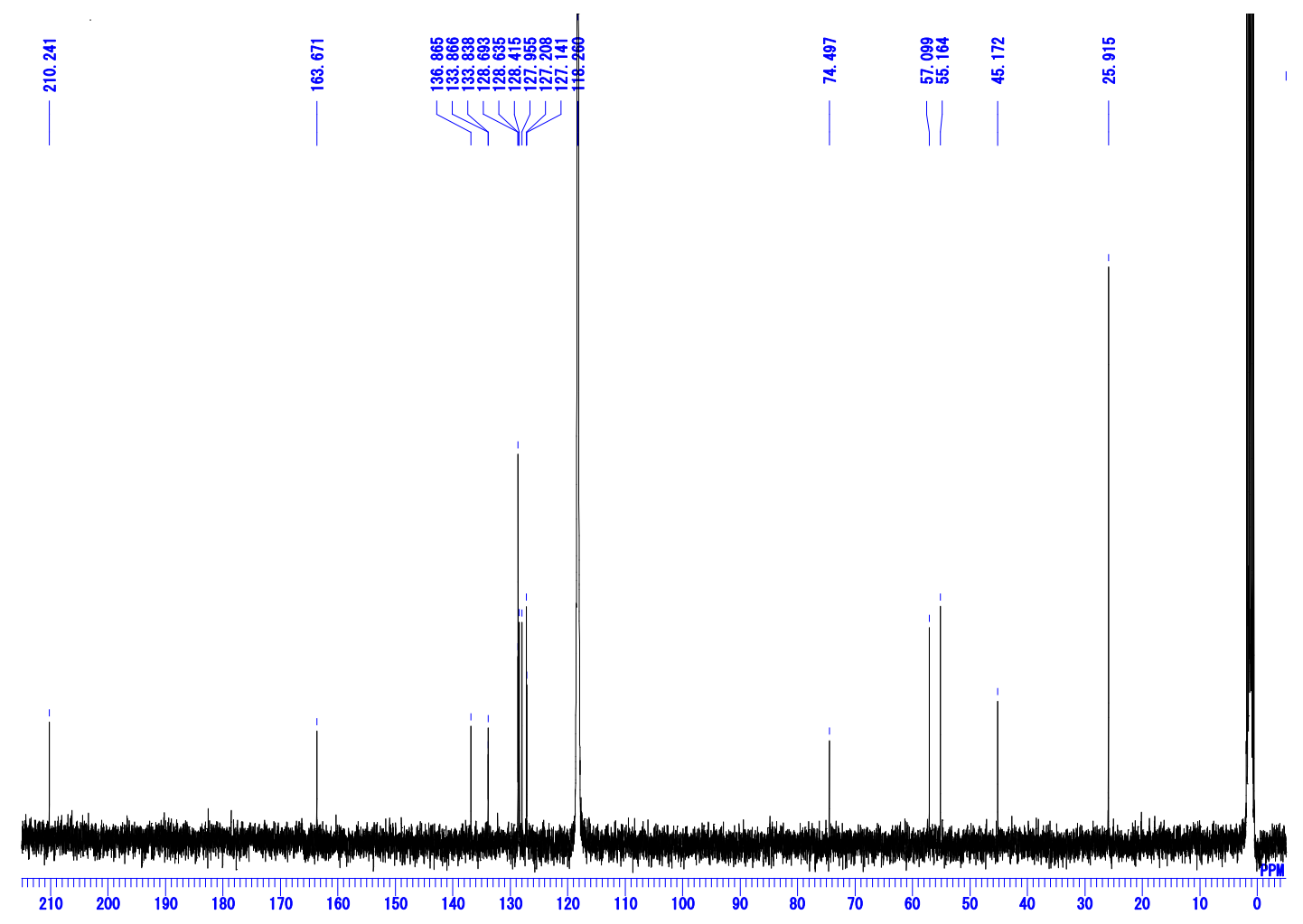

MW Profile

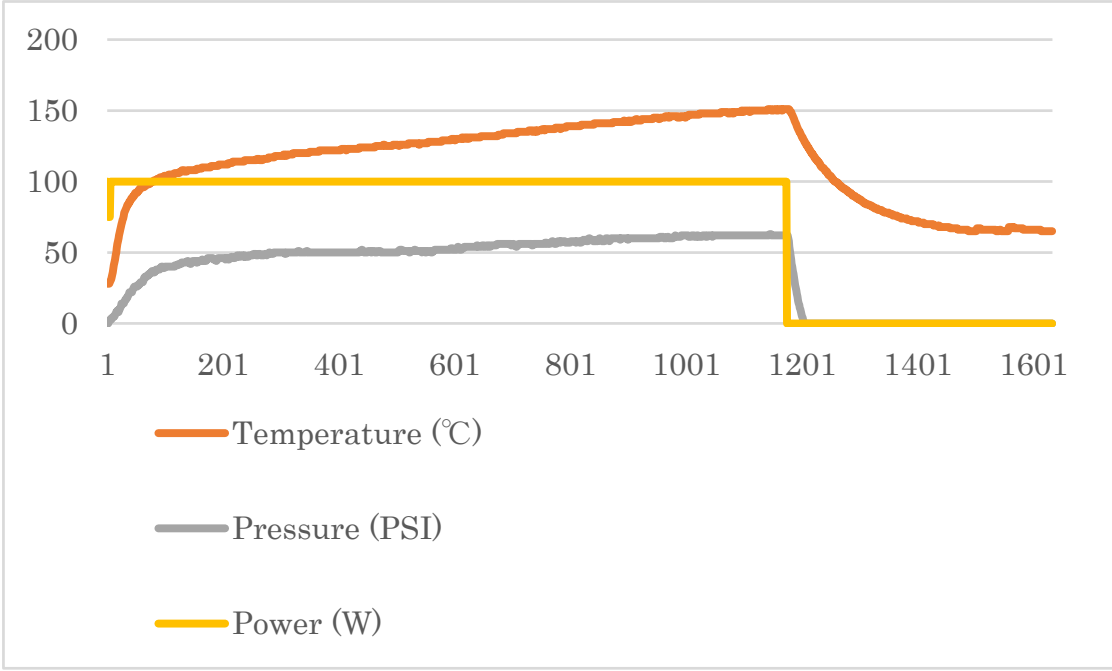


2-Amino-4-(furan-2-yl)-5-pivaloyl-4,5-dihydrothiophene-3-carbonitrile (trans-3bf)<smiles>CC(C)(C)C(=O)[C@H]1SC(N)=C(C#N)[C@H]1c1ccco1</smiles>

${ }^{1} \mathrm{H}$ NMR

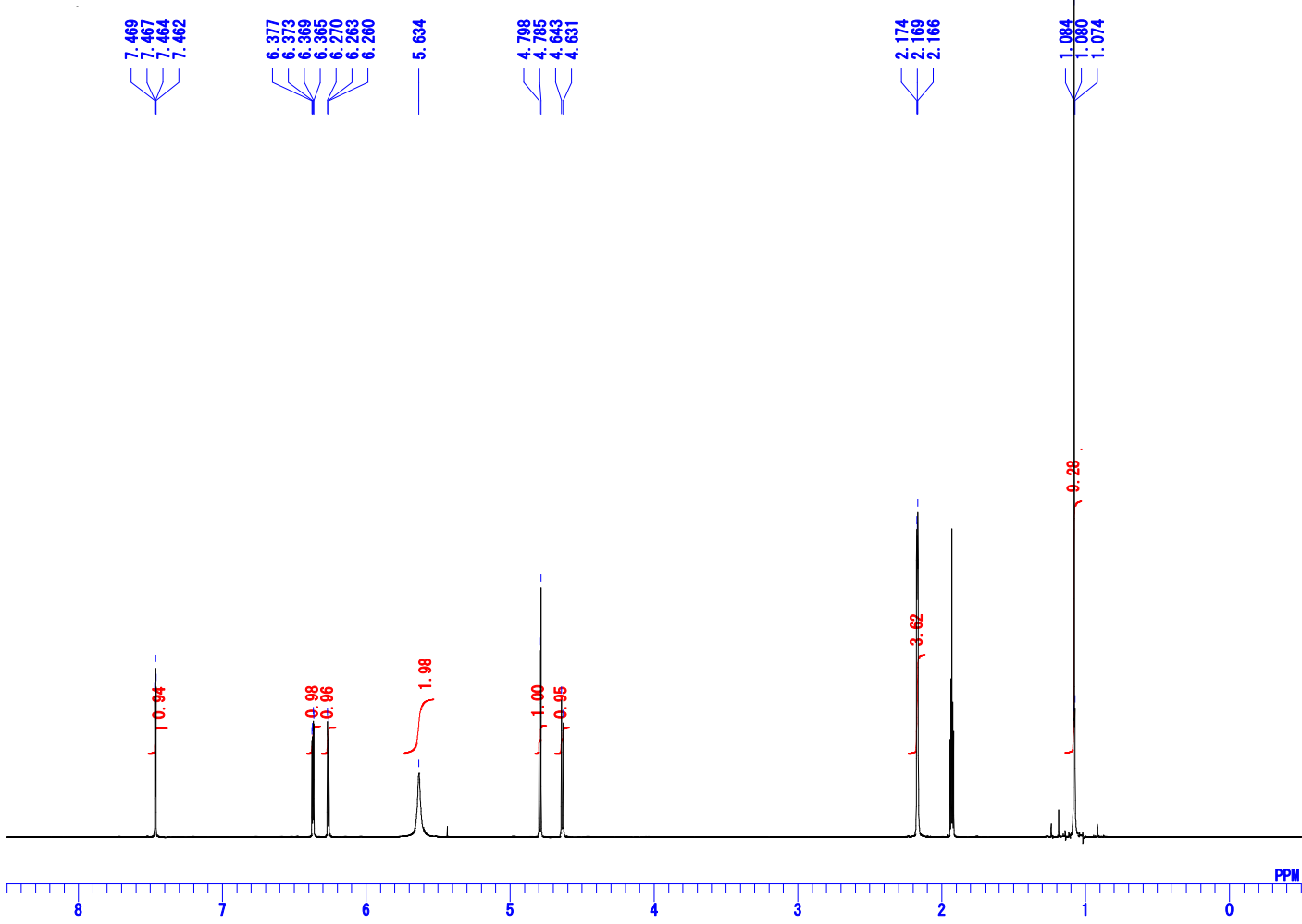


${ }^{13} \mathrm{C}$ NMR

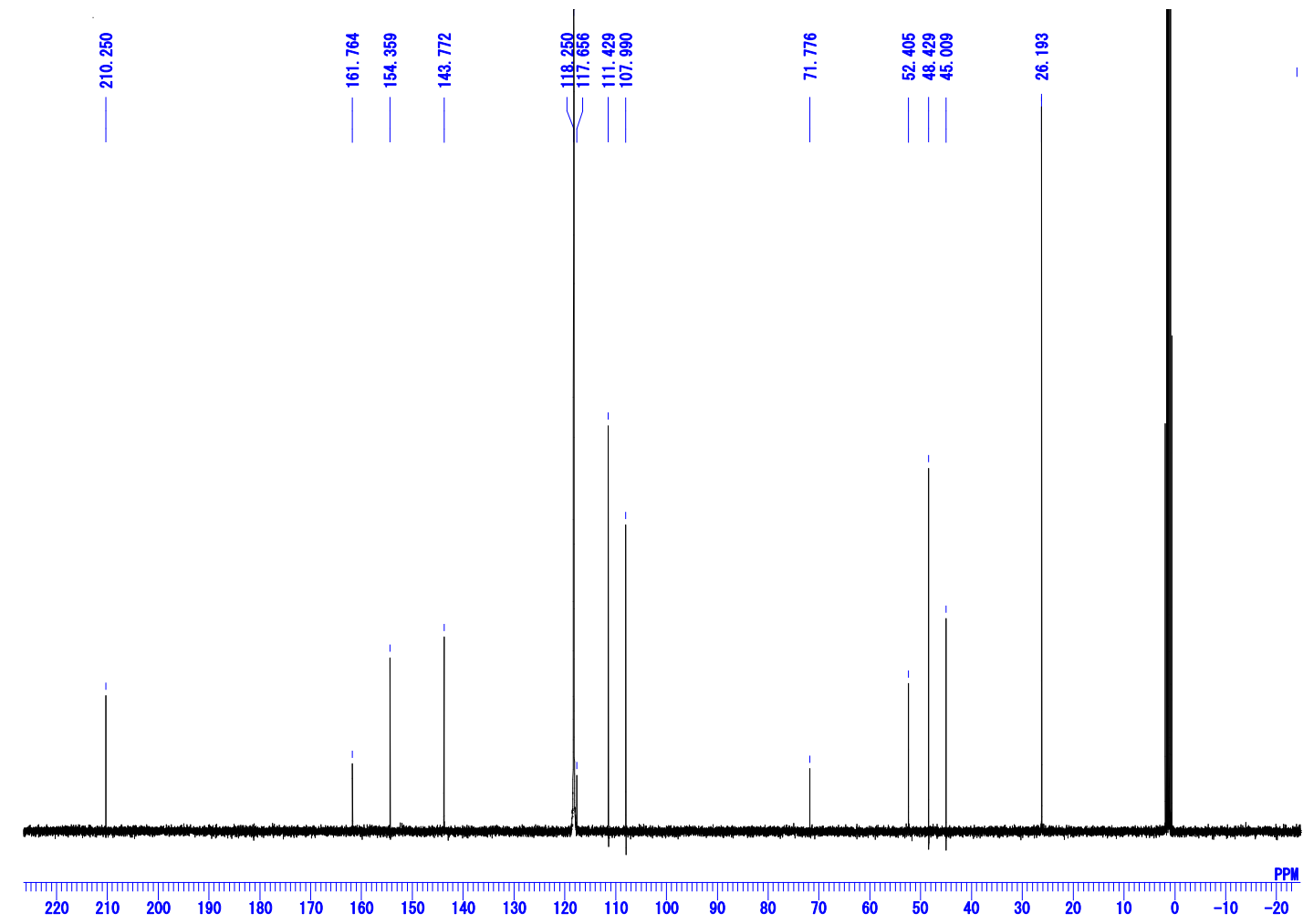

MW Profile

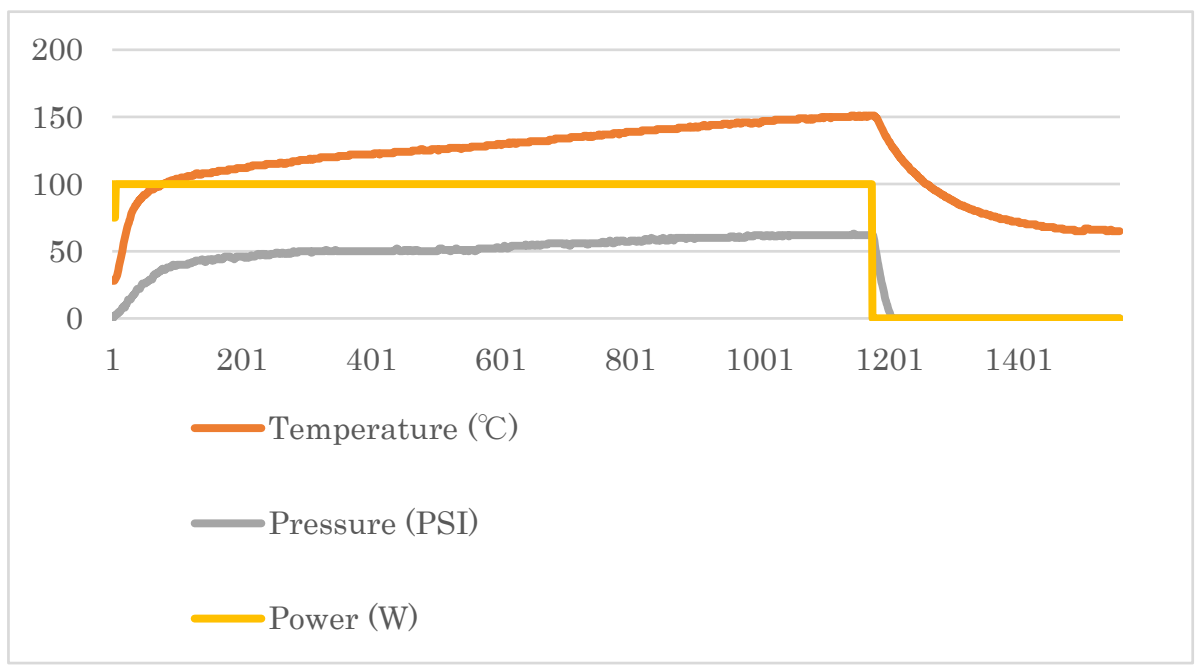


2-Amino-4-(furan-2-yl)-5-pivaloyl-4,5-dihydrothiophene-3-carbonitrile (cis-3bf)<smiles>CC(C)(C)C(=O)[C@H]1SC(N)=C(C#N)[C@H]1c1ccco1</smiles>

${ }^{1} \mathrm{H}$ NMR

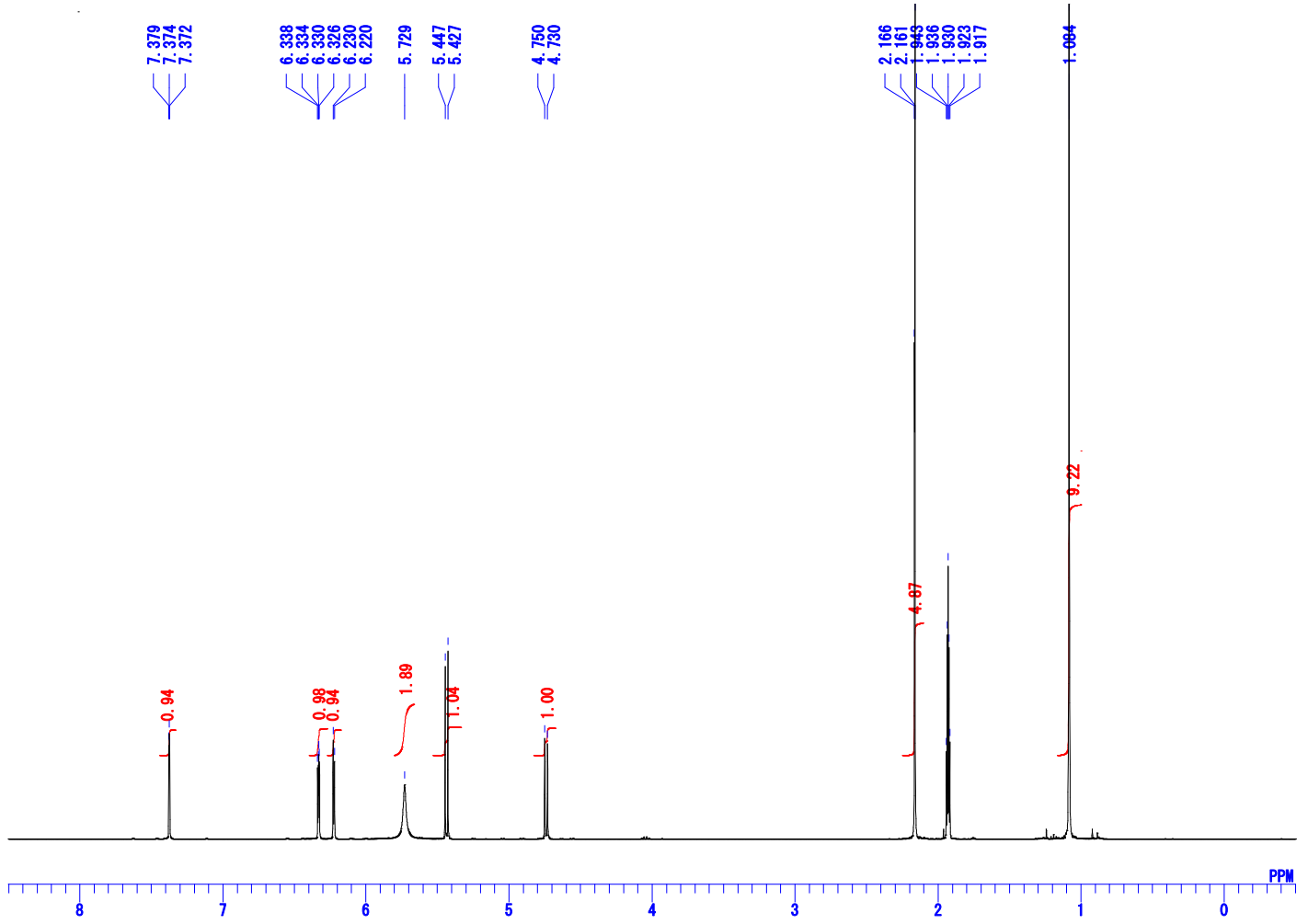


${ }^{13} \mathrm{C}$ NMR

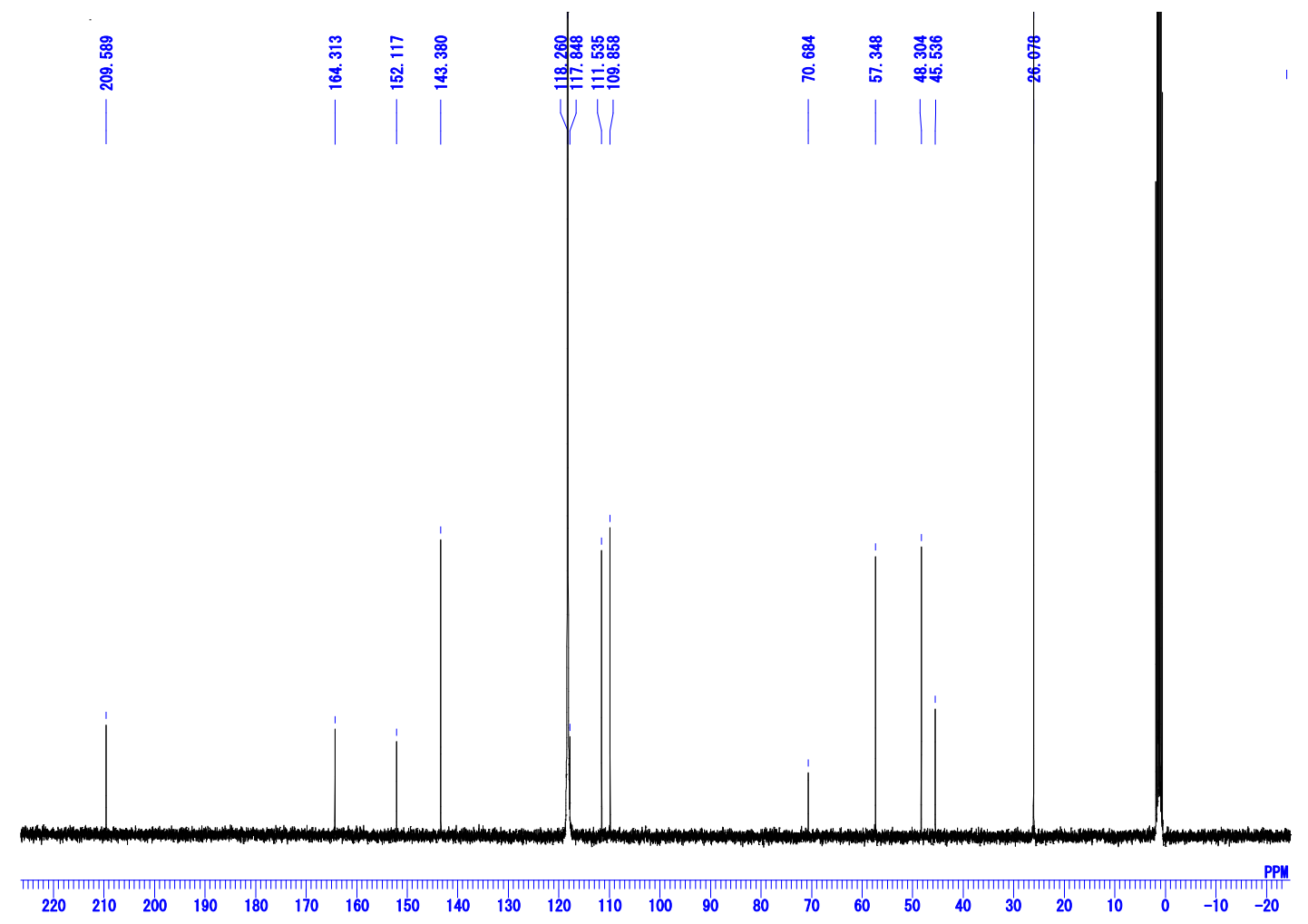

MW Profile

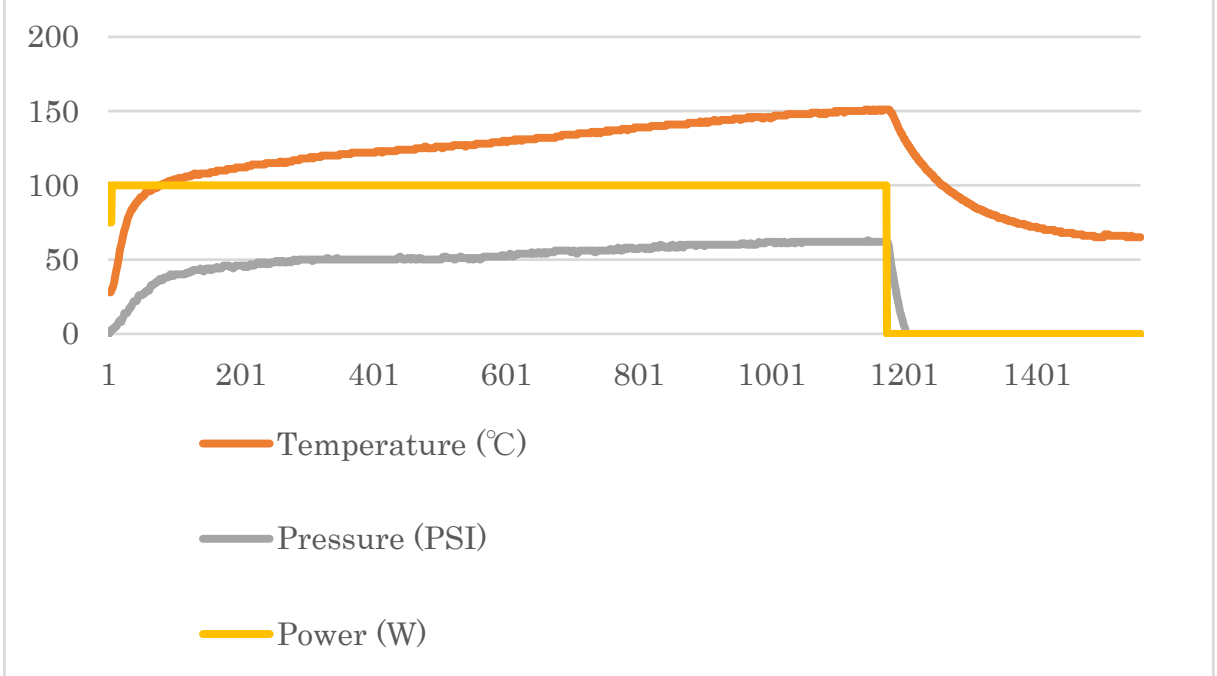


2-Amino-4-phenethyl-5-pivaloyl-4,5-dihydrothiophene-3-carbonitrile (trans-3bg)

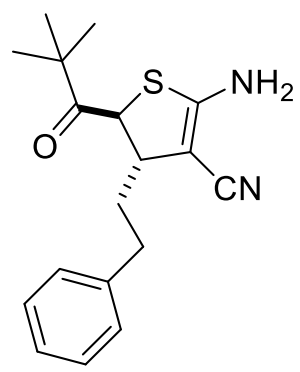

${ }^{1} \mathrm{H}$ NMR

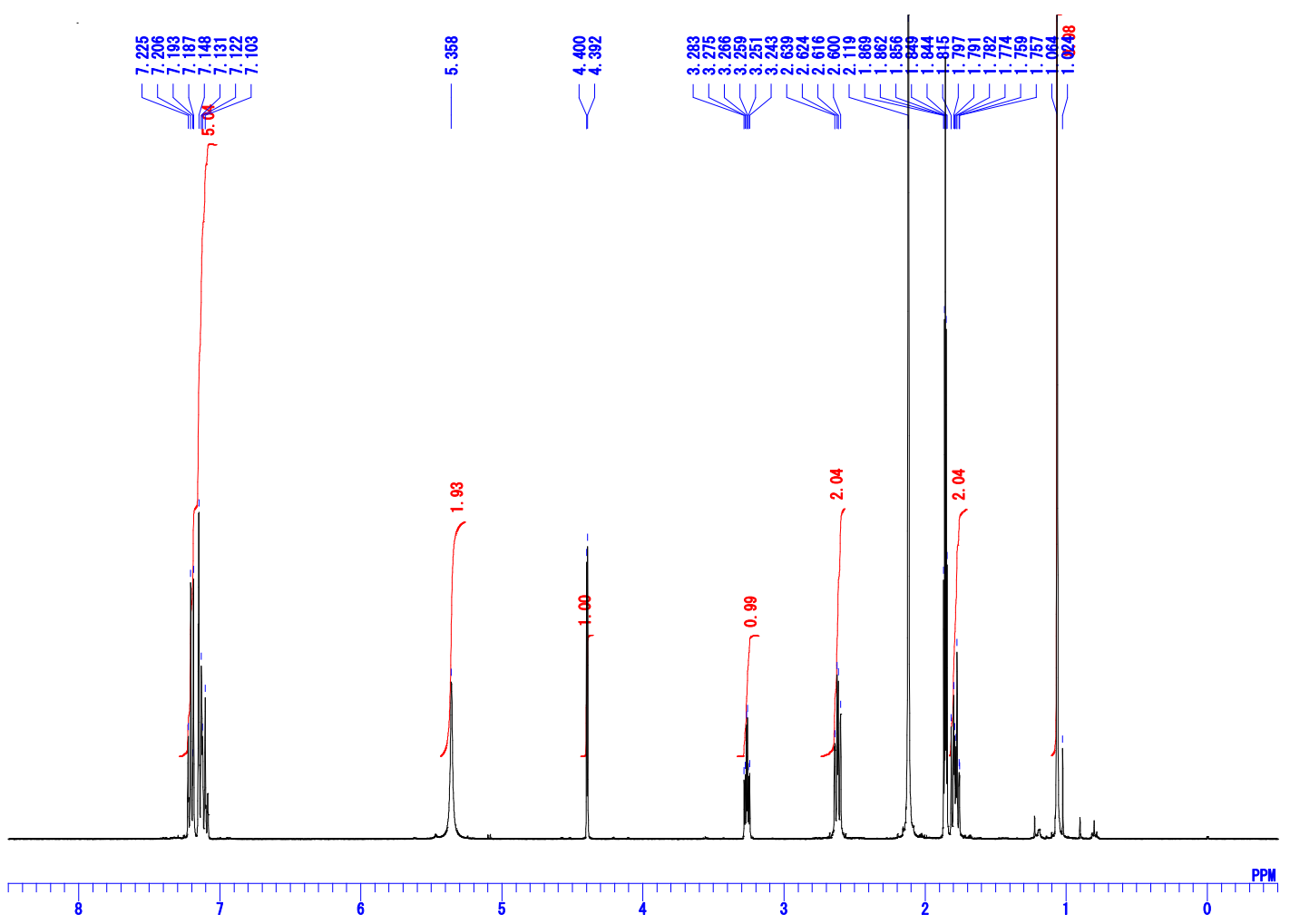


${ }^{13} \mathrm{C}$ NMR

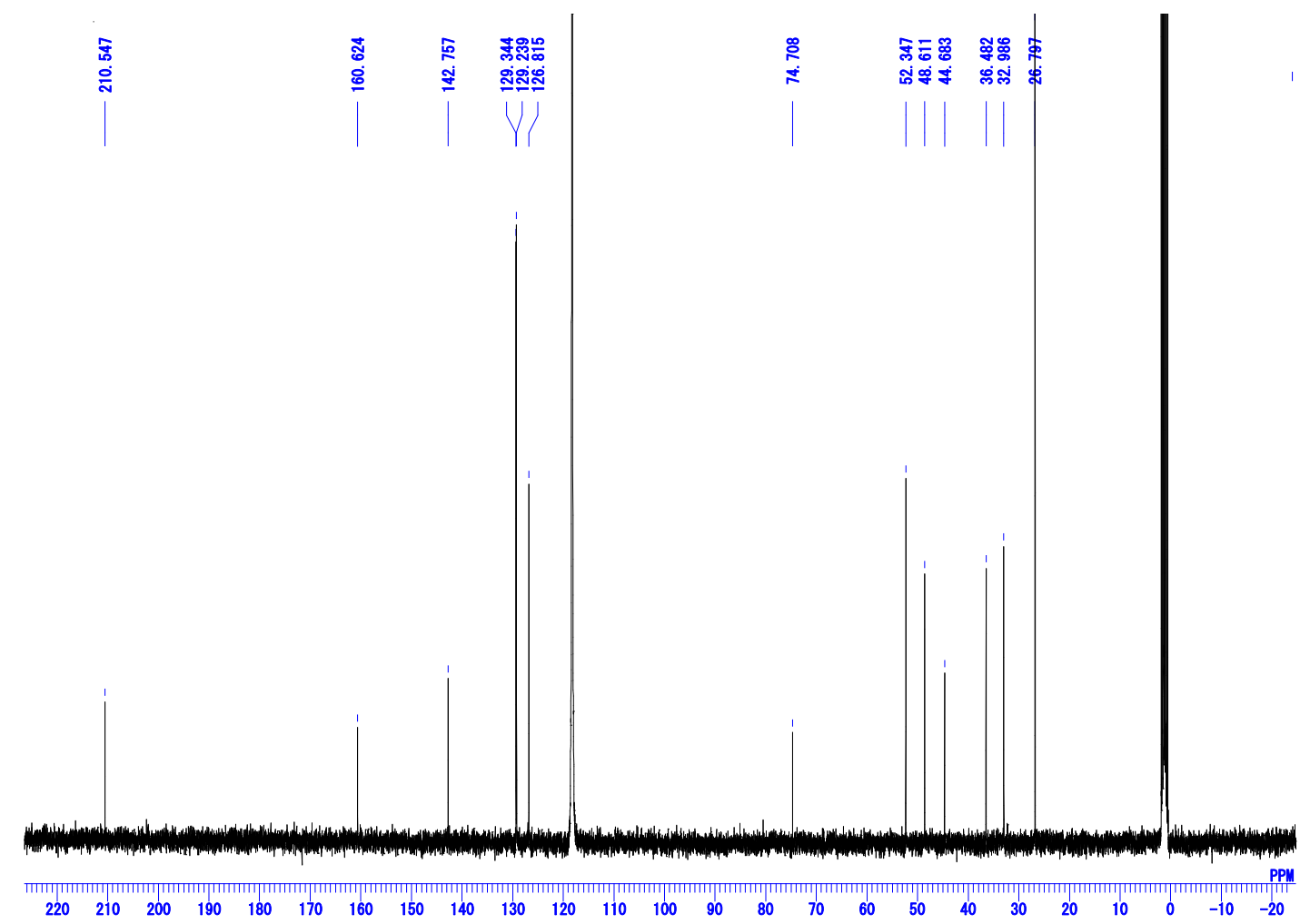

MW Profile

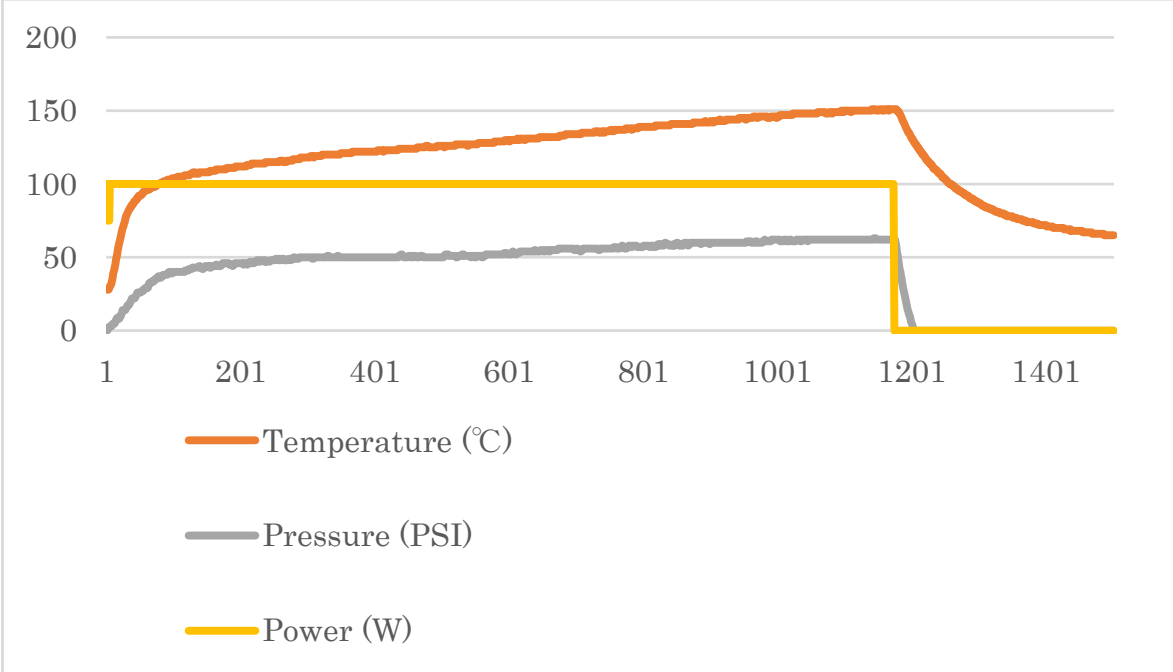


2-Amino-4-phenethyl-5-pivaloyl-4,5-dihydrothiophene-3-carbonitrile (cis-3bg)<smiles>CC(C)(C)C(=O)C1SC(N)=C(C#N)C1CCc1ccccc1</smiles>

${ }^{1} \mathrm{H}$ NMR

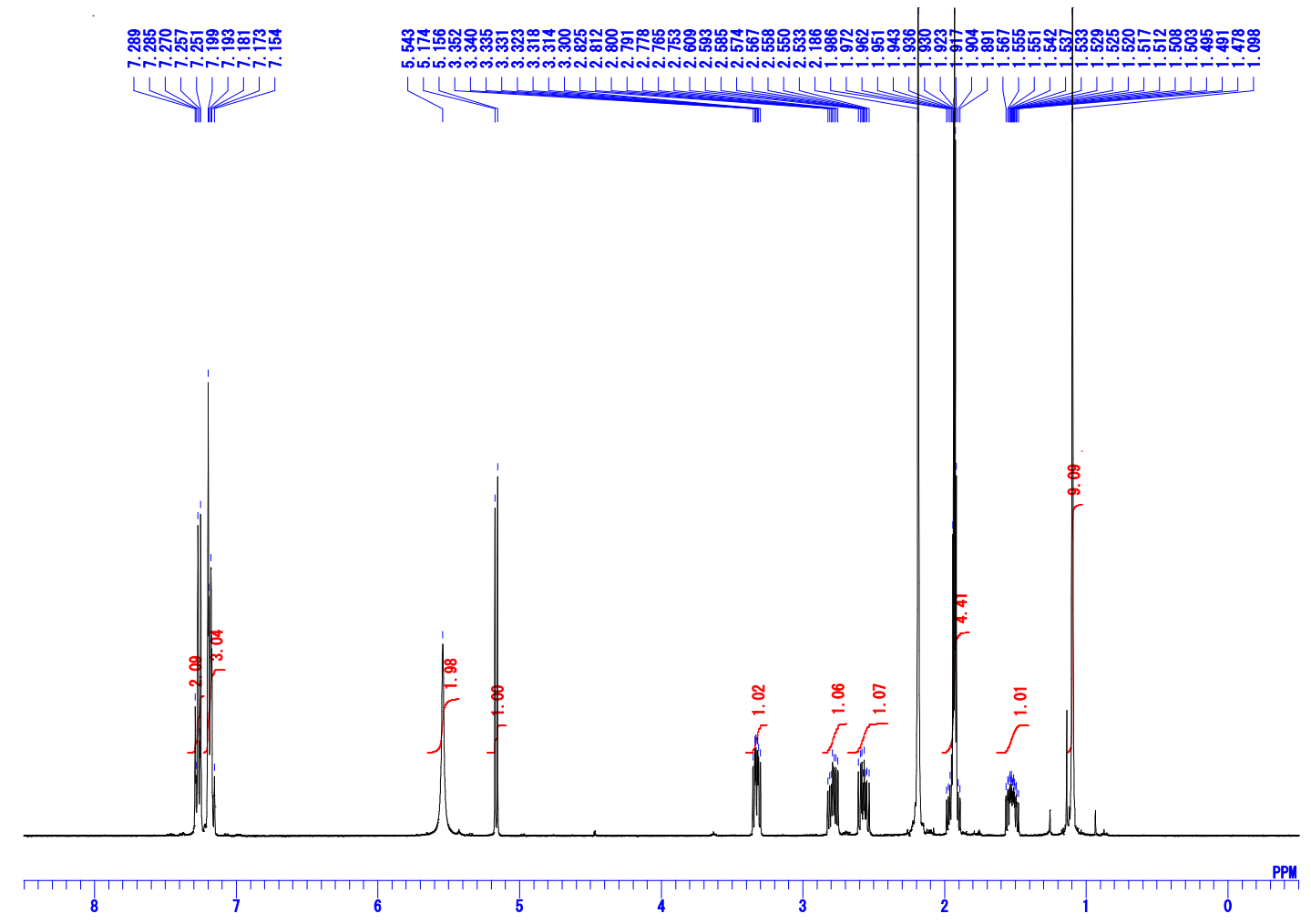


${ }^{13} \mathrm{C}$ NMR

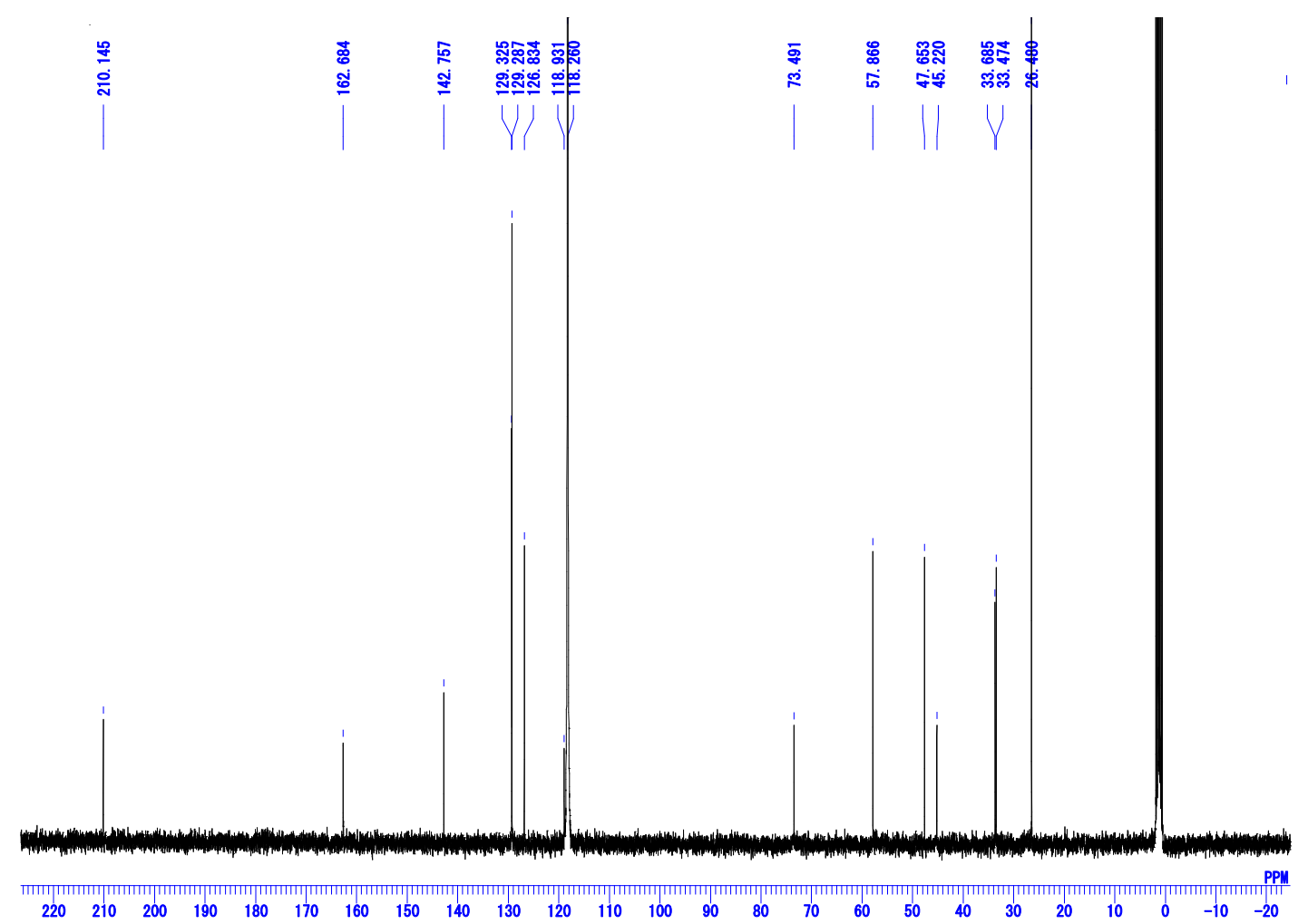

MW Profile

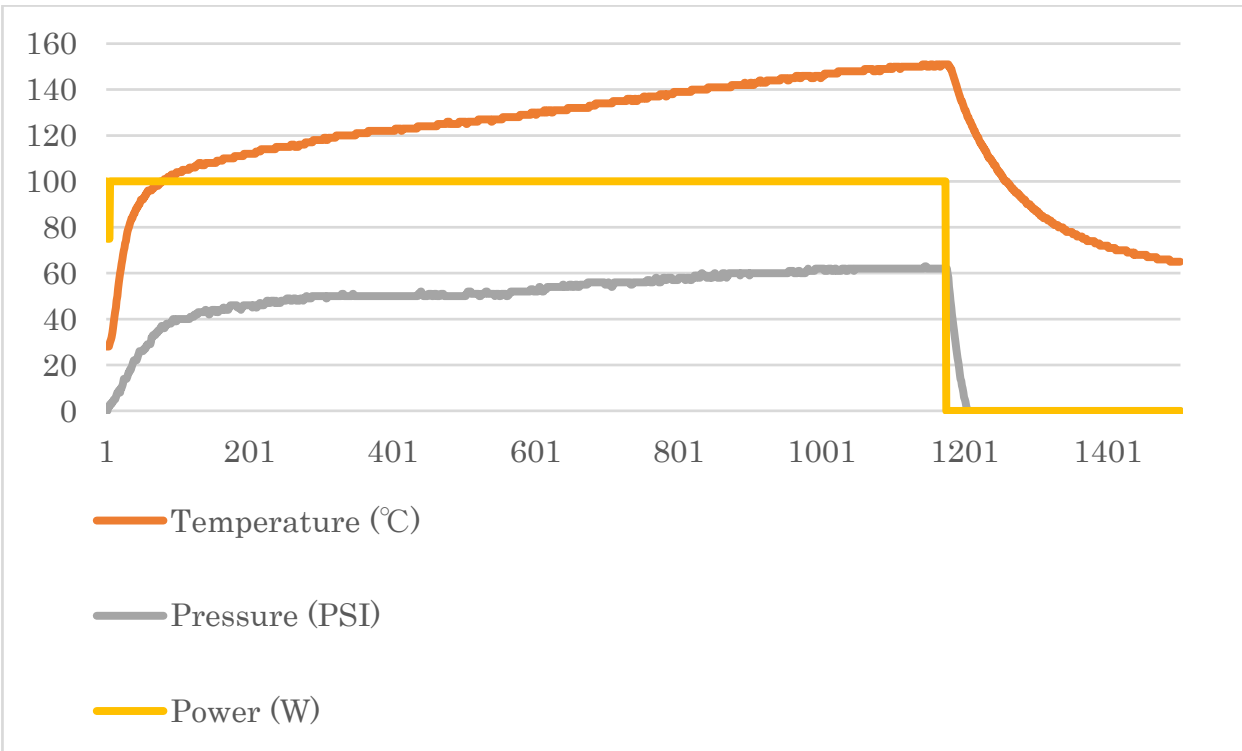


2-Amino-5-benzoyl-4-phenyl-4,5-dihydrothiophene-3-carbonitrile (trans-3ca)

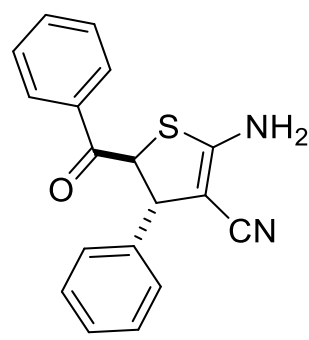

${ }^{1} \mathrm{H}$ NMR

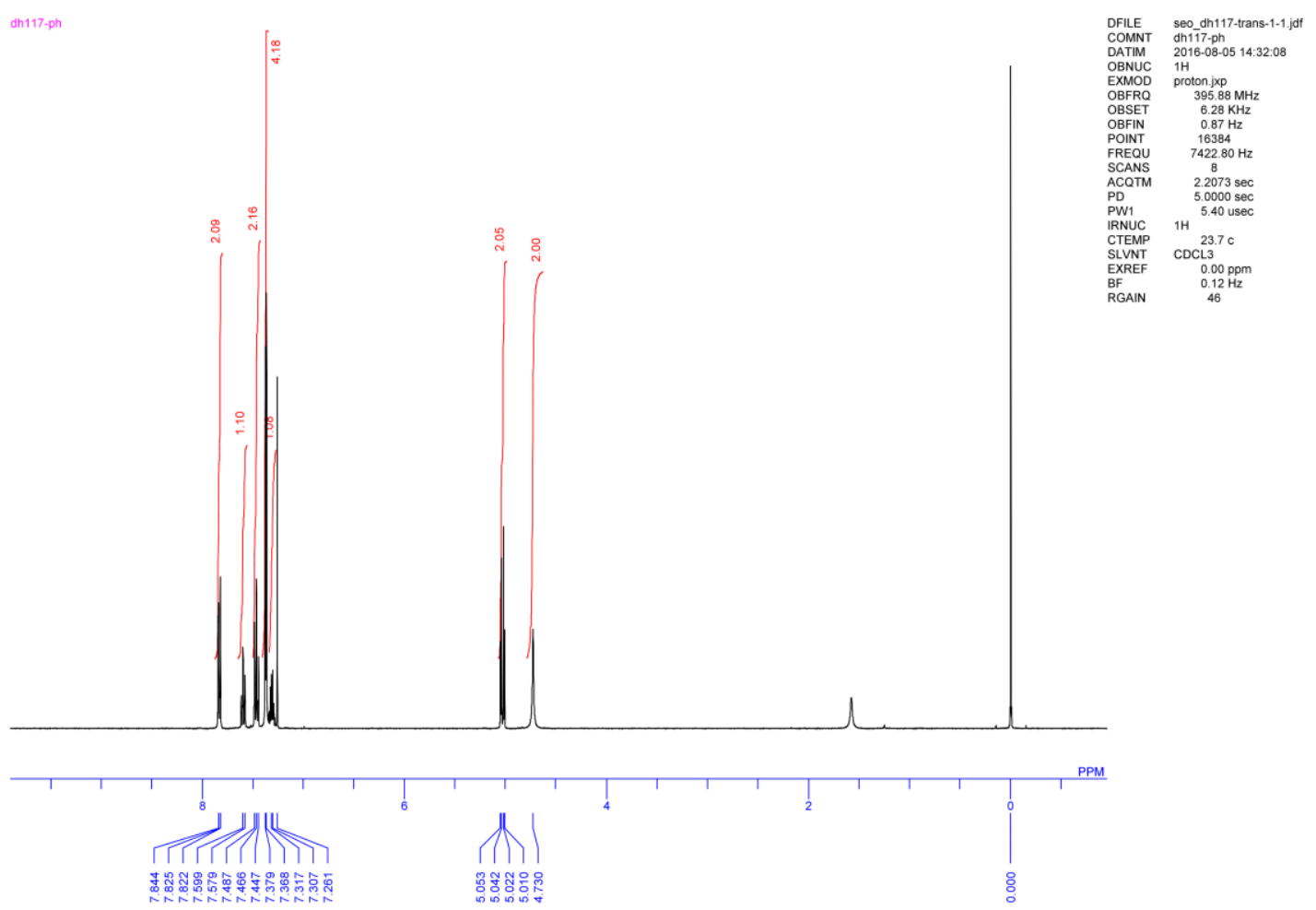


${ }^{13} \mathrm{C}$ NMR

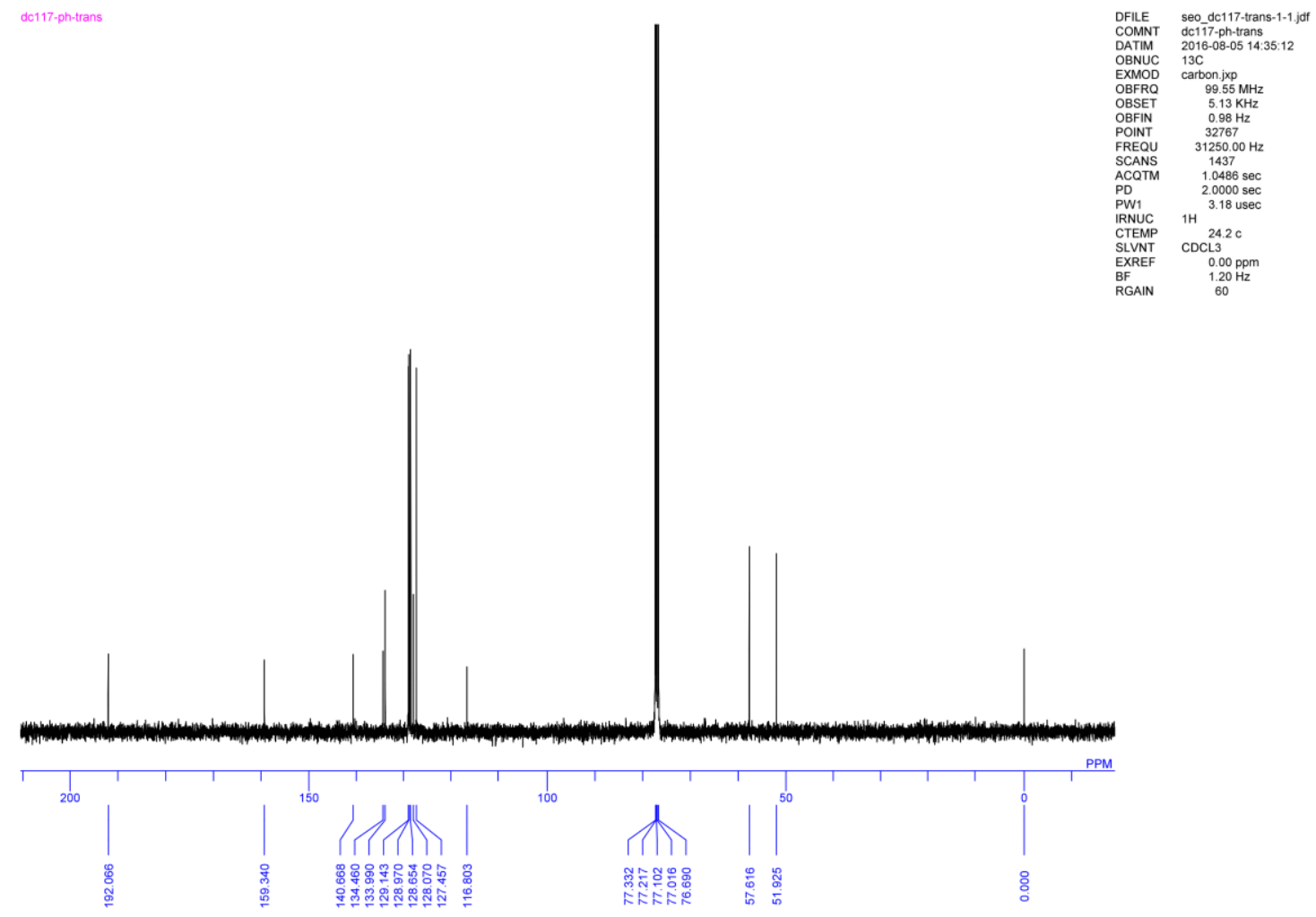

MW Profile

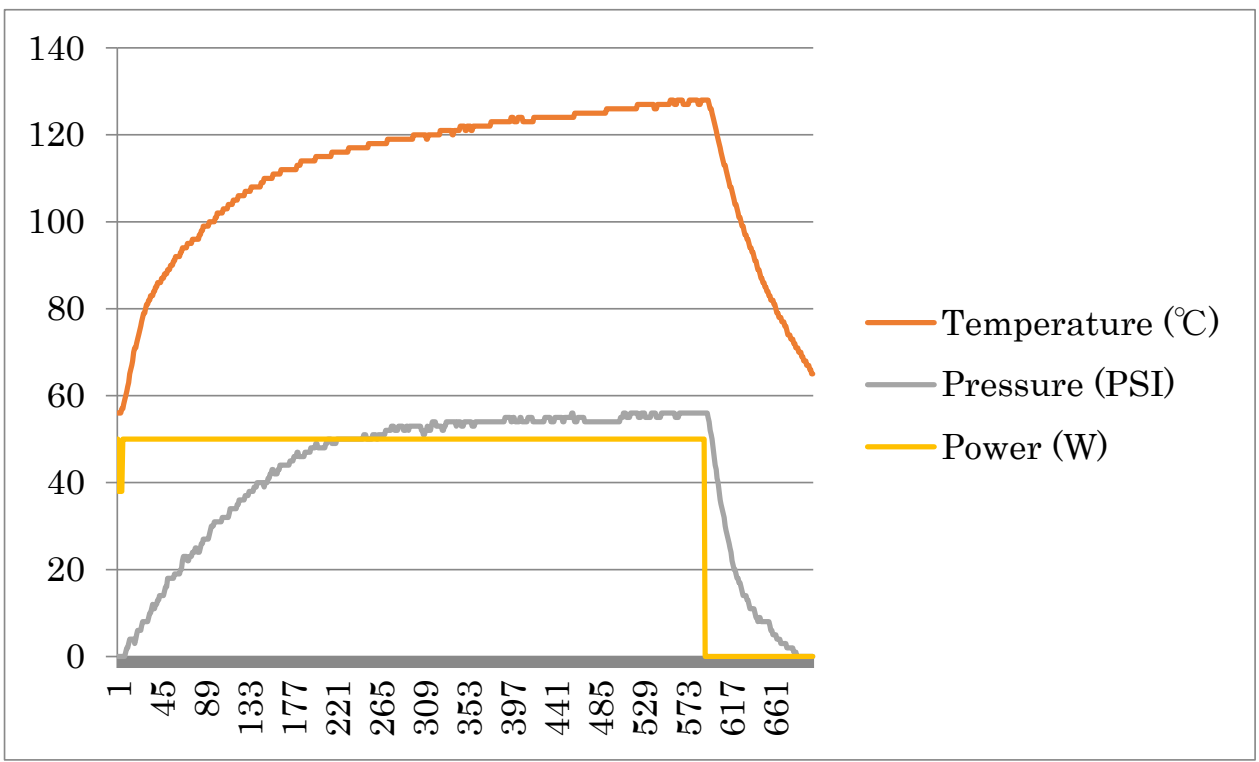


2-Amino-5-benzoyl-4-phenyl-4,5-dihydrothiophene-3-carbonitrile (cis-3ca)

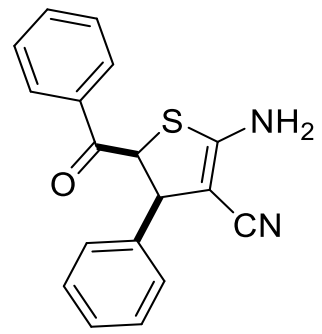

${ }^{1} \mathrm{H}$ NMR

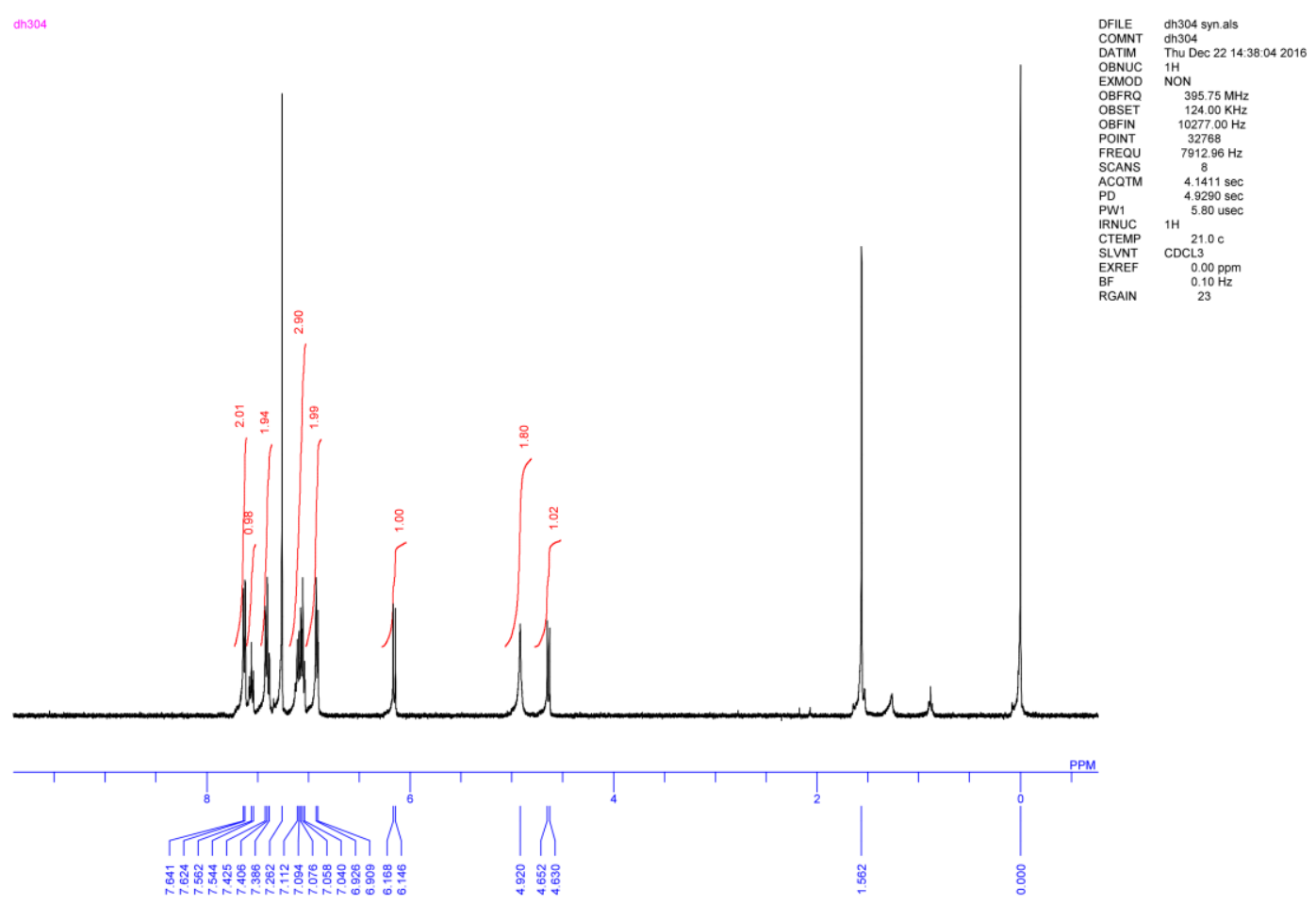


${ }^{13} \mathrm{C}$ NMR

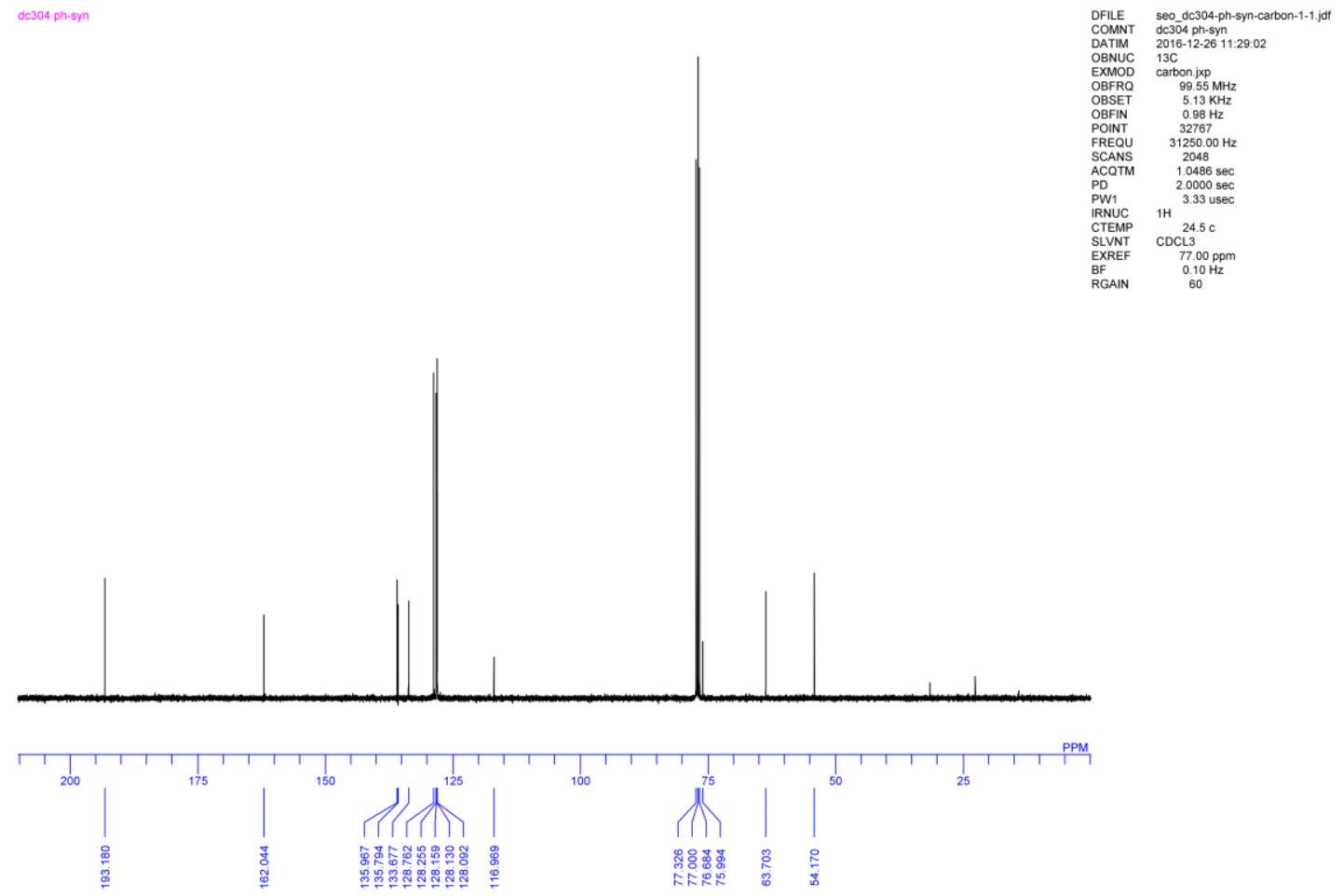


2-Amino-5-benzoyl-4-(4-chlorophenyl)-4,5-dihydrothiophene-3-carbonitrile (trans-3cb)

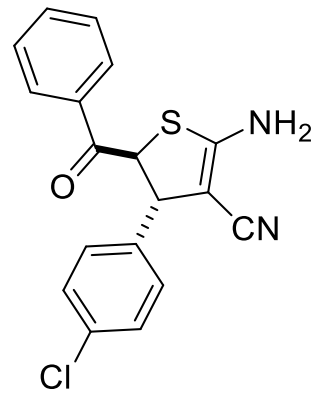

${ }^{1} \mathrm{H}$ NMR

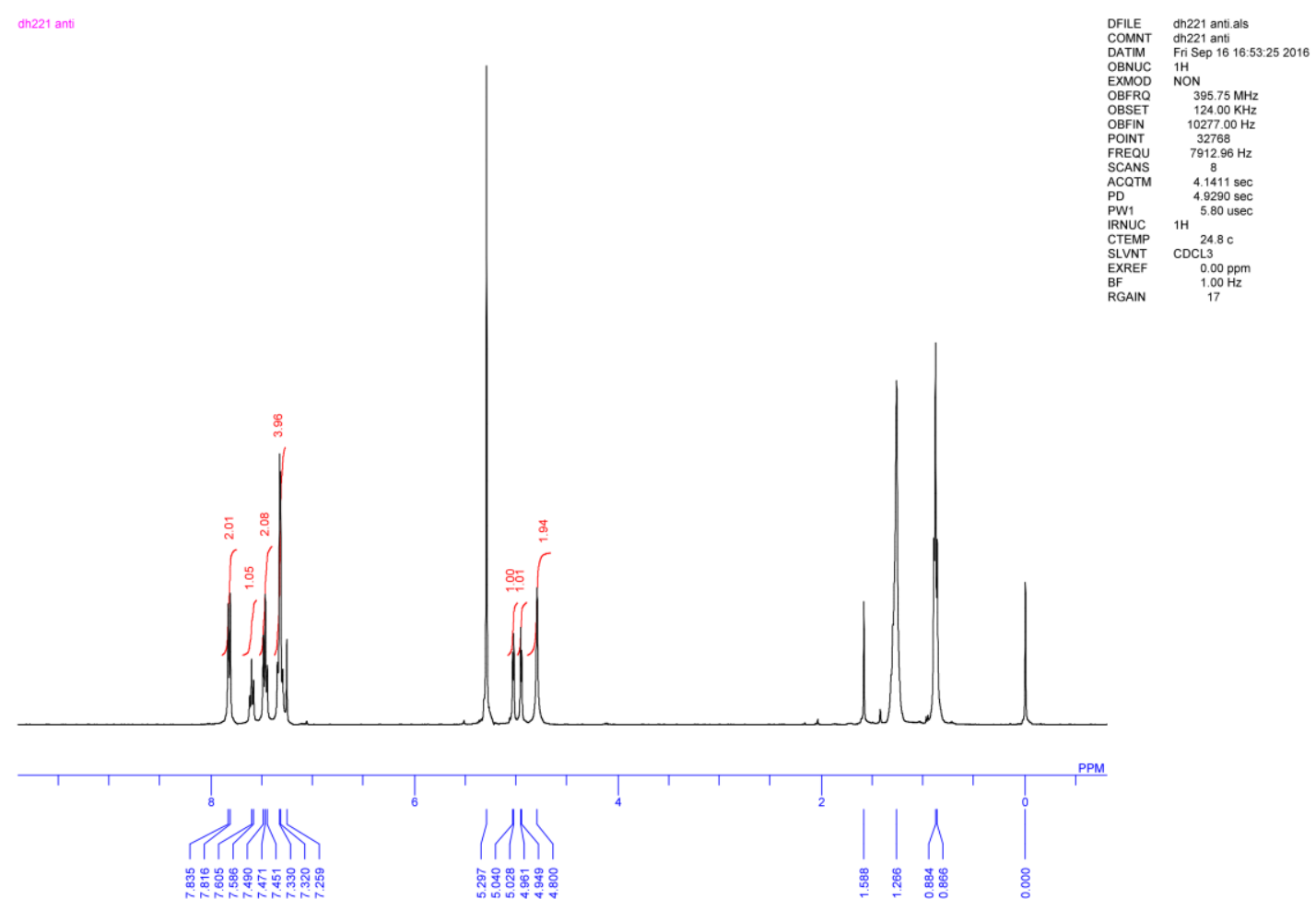


${ }^{13} \mathrm{C}$ NMR

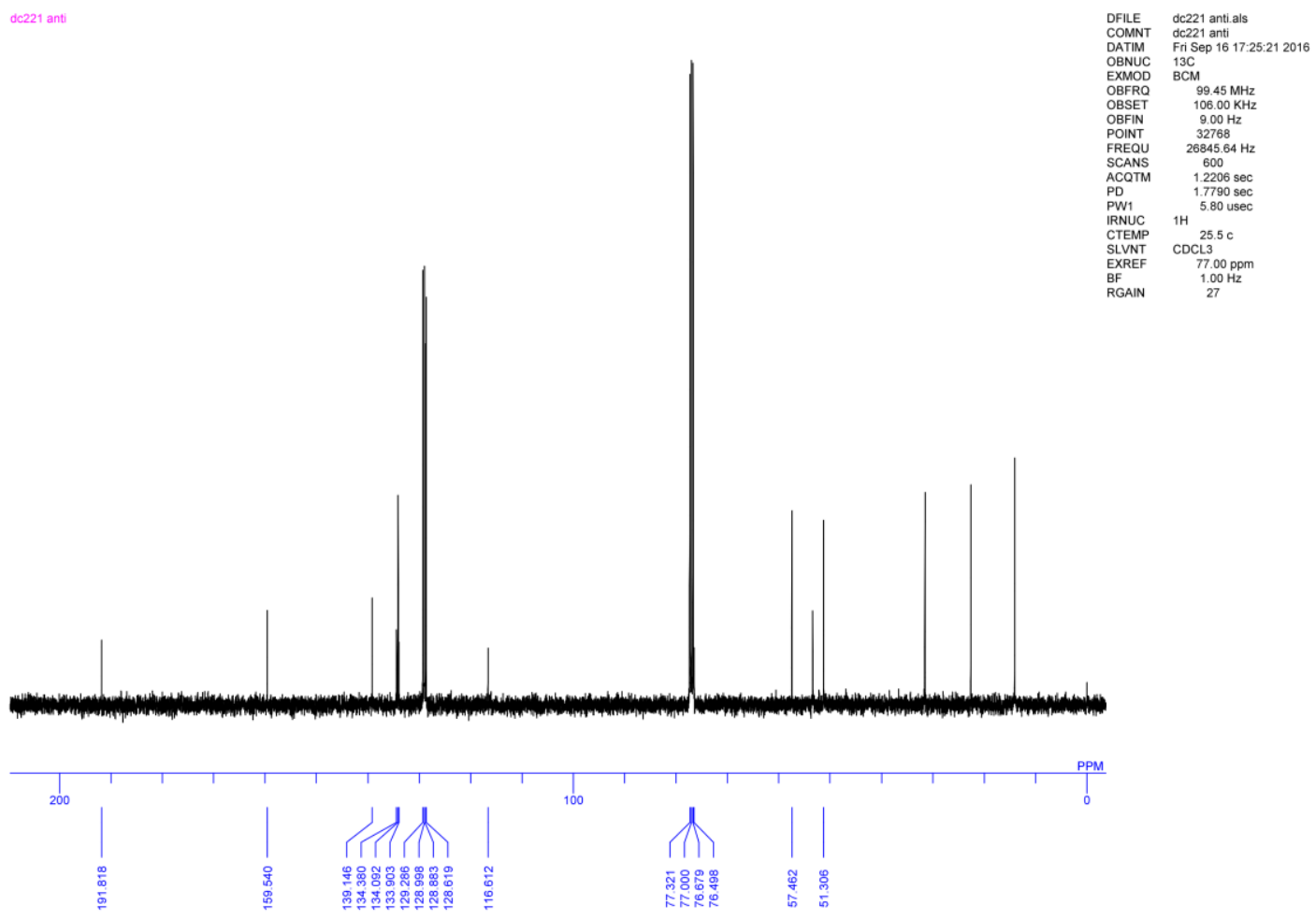

MW Profile

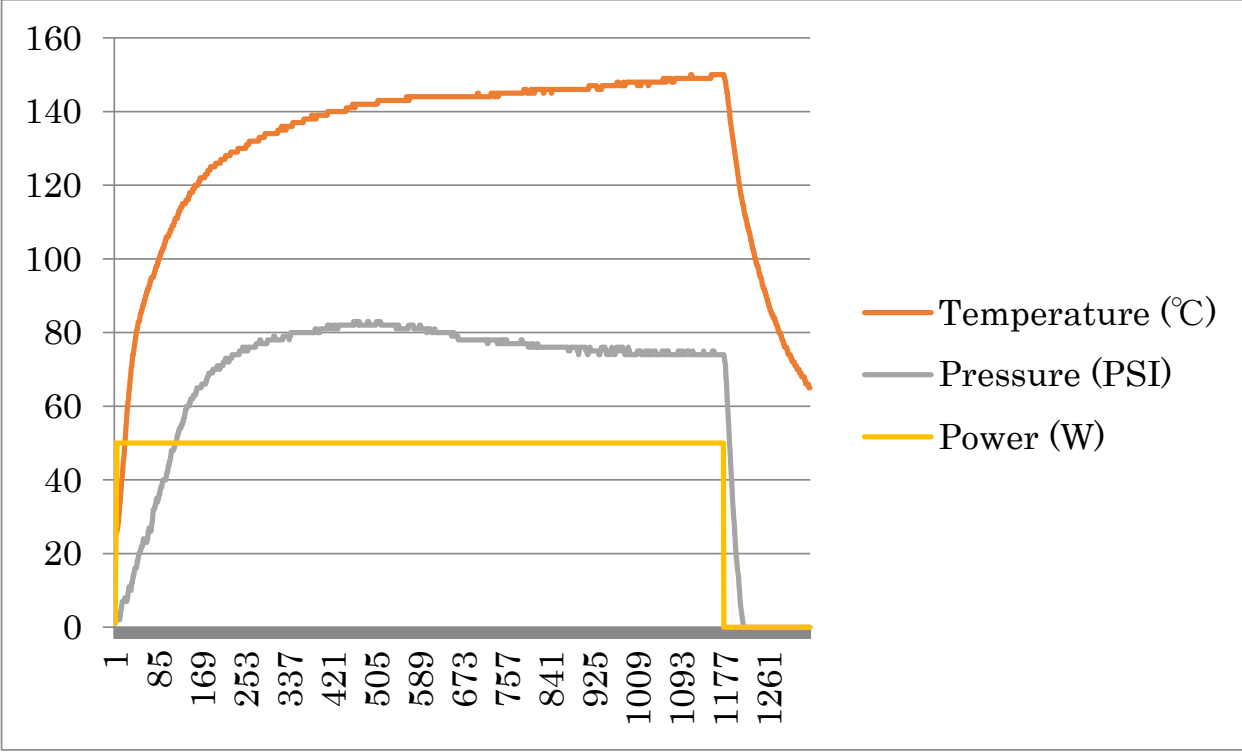


2-Amino-5-benzoyl-4-(4-chlorophenyl)-4,5-dihydrothiophene-3-carbonitrile (cis-3cb)

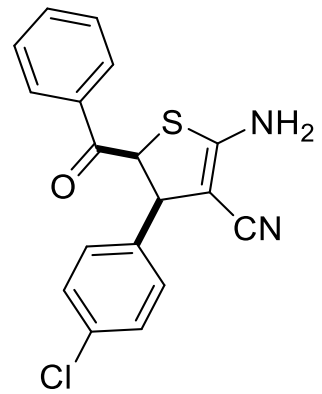

${ }^{1} \mathrm{H}$ NMR

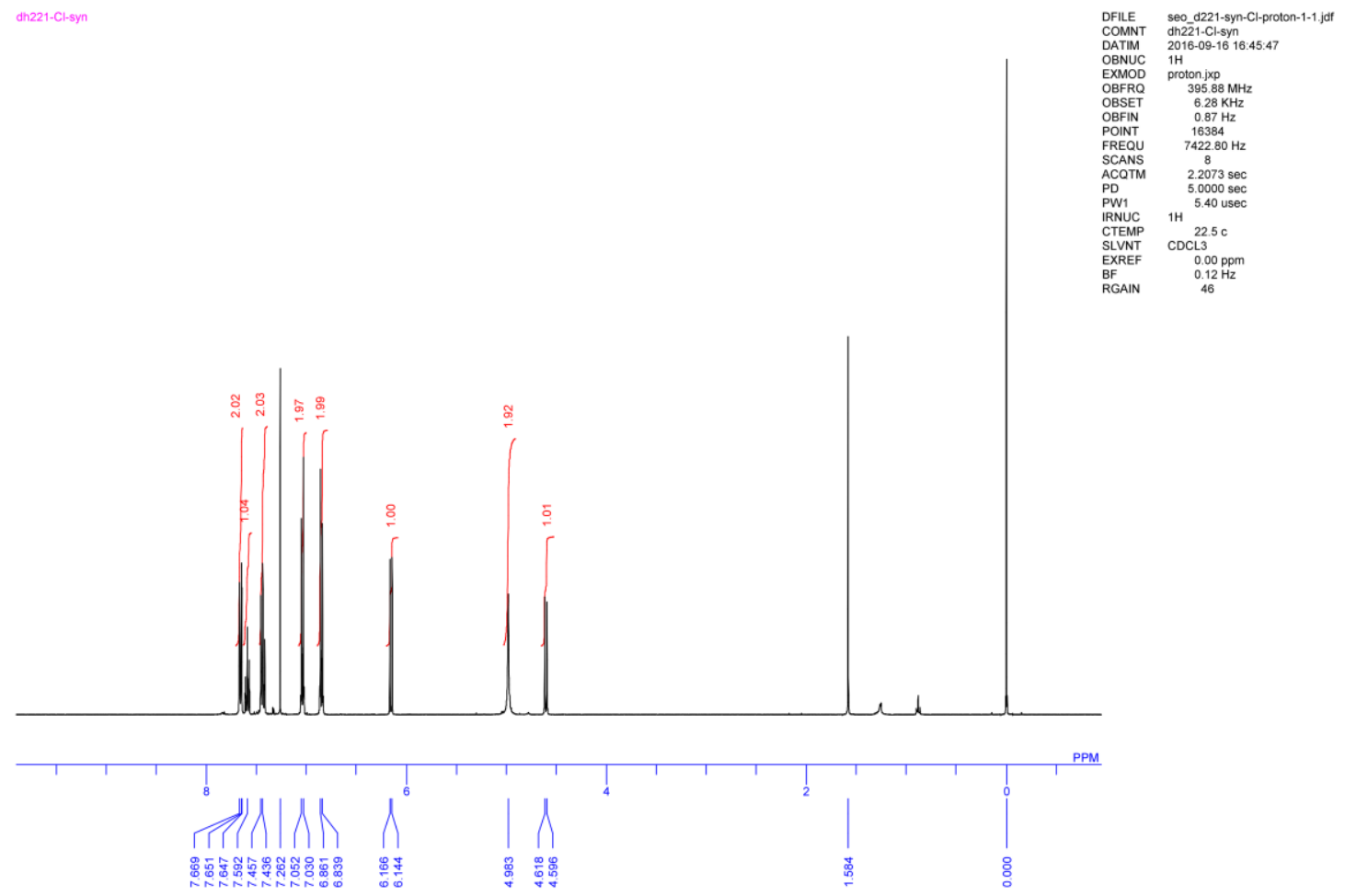


${ }^{13} \mathrm{C}$ NMR

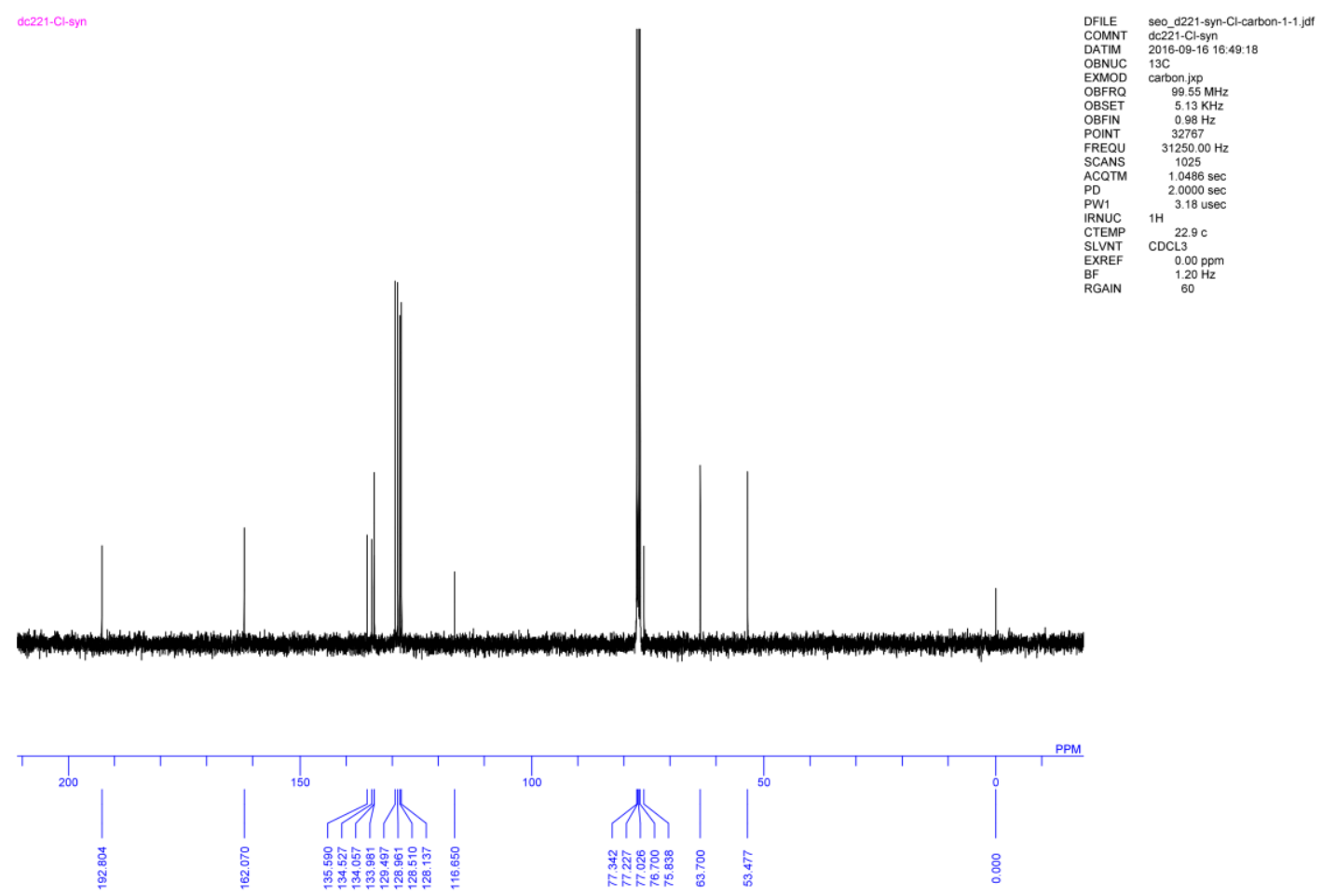


2-Amino-5-benzoyl-4-(naphthalen-1-yl)-4,5-dihydrothiophene-3-carbonitrile (trans-3ce)

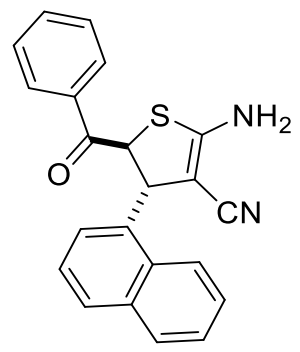

${ }^{1} \mathrm{H}$ NMR

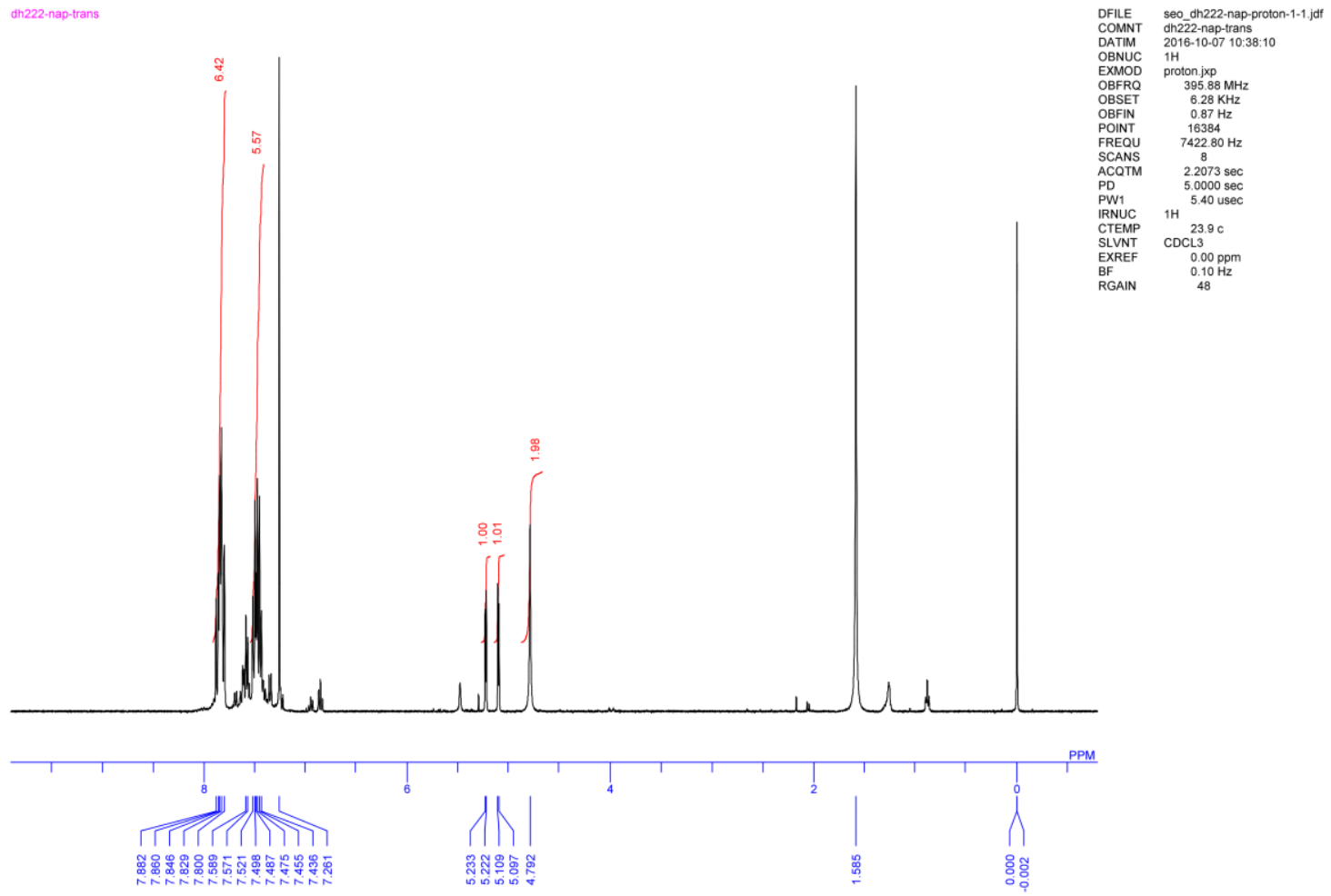


${ }^{13} \mathrm{C}$ NMR

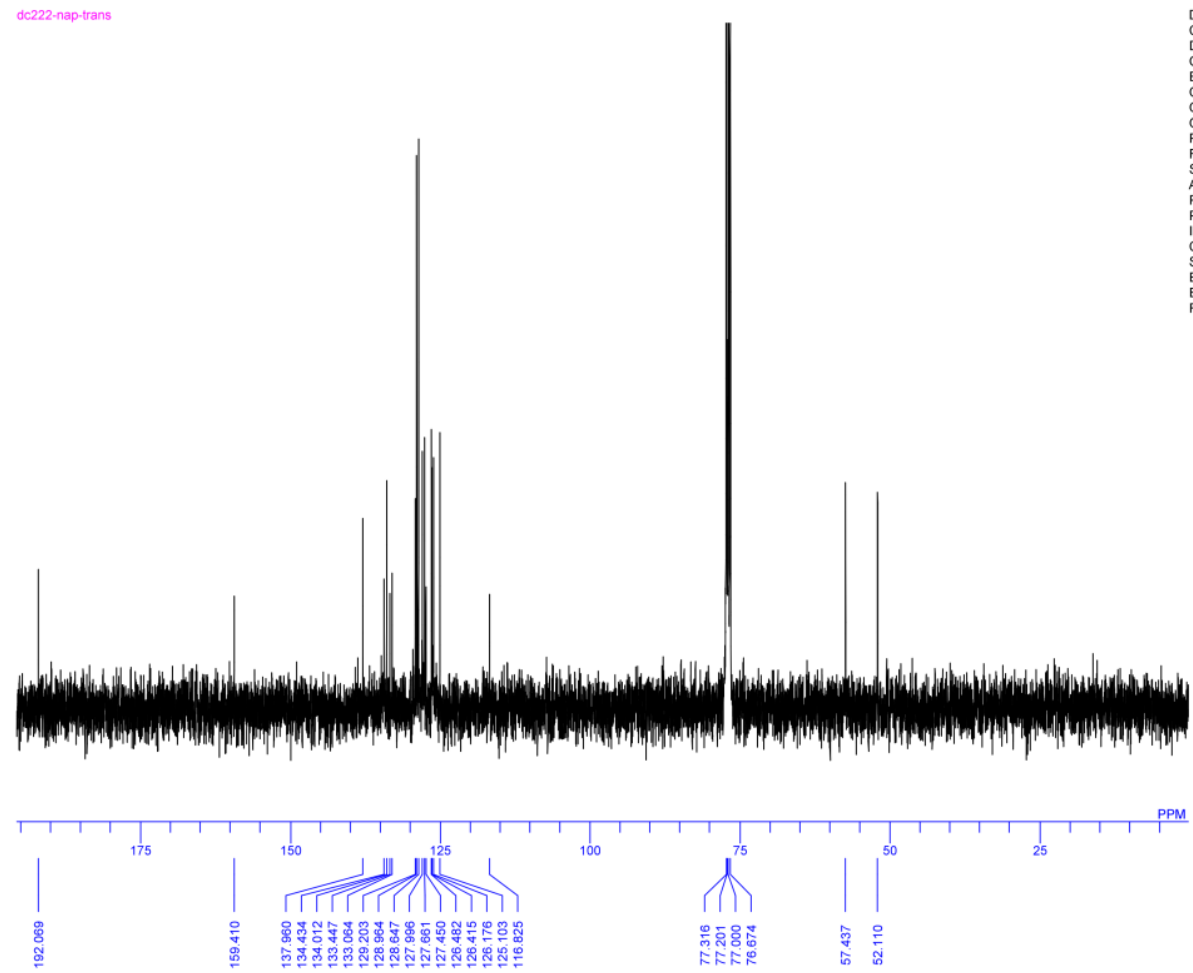

MW Profile

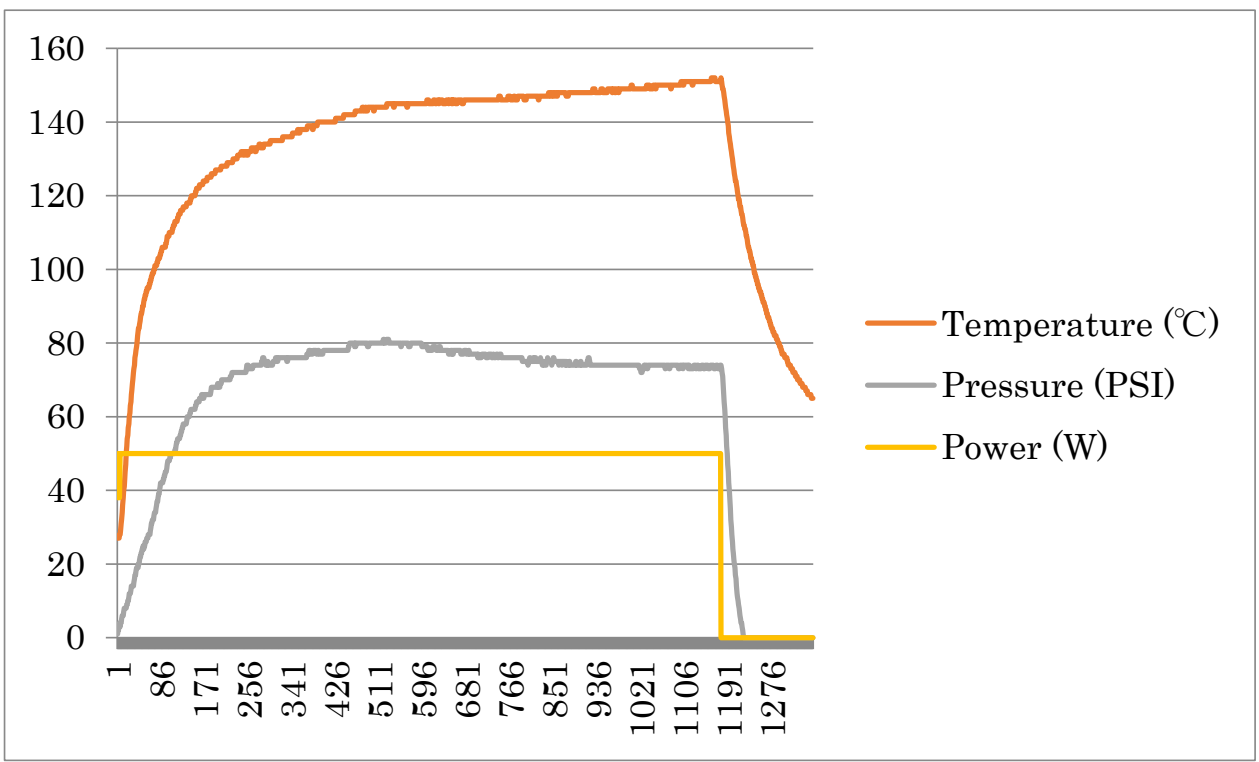


2-Amino-5-benzoyl-4-(naphthalen-1-yl)-4,5-dihydrothiophene-3-carbonitrile (cis-3ce)

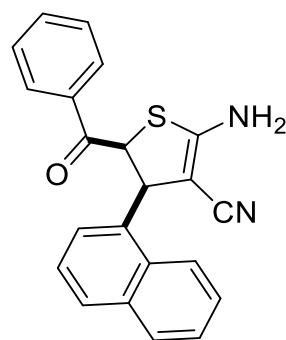

${ }^{1} \mathrm{H}$ NMR

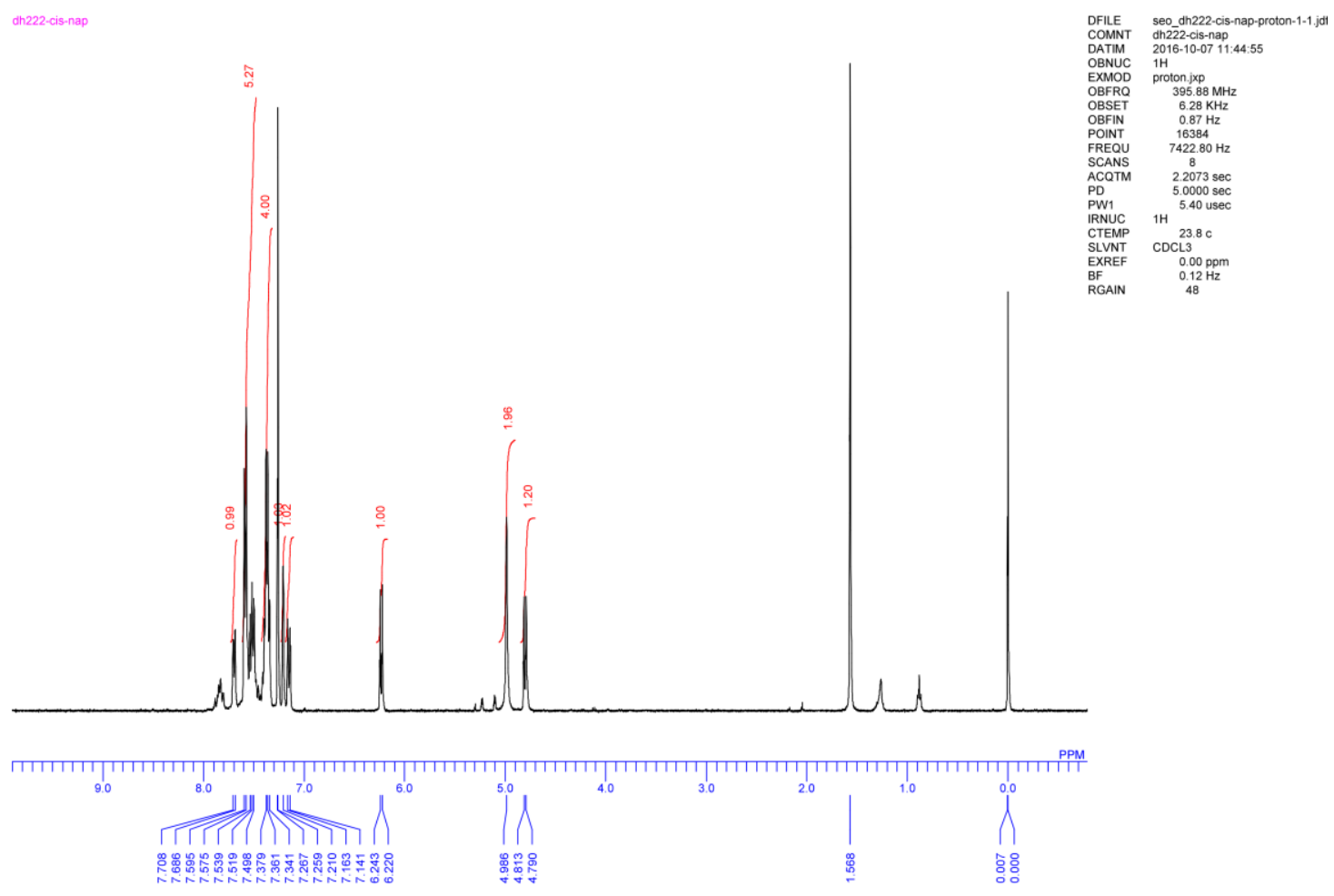


${ }^{13} \mathrm{C}$ NMR

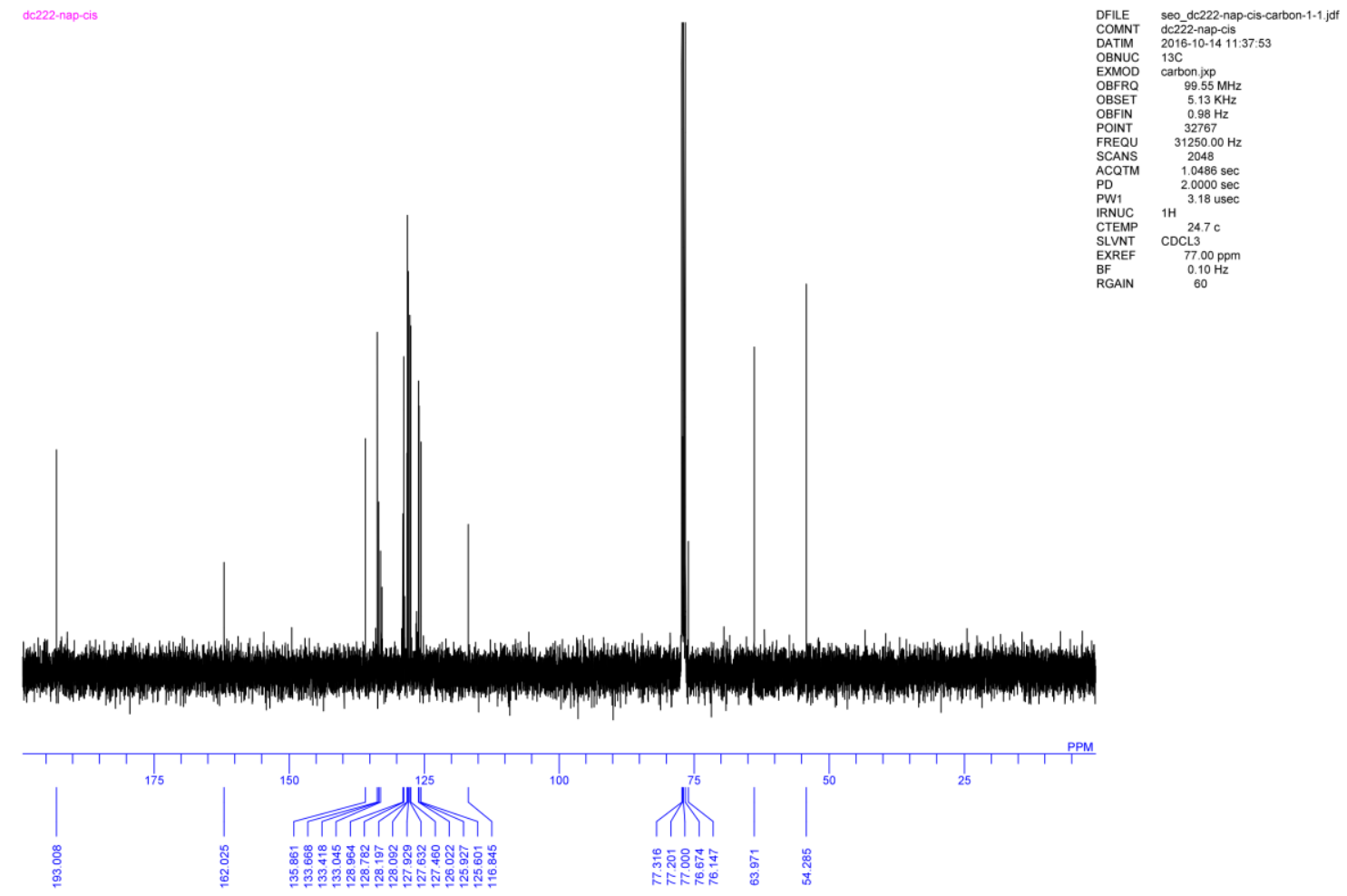


2-Amino-5-benzoyl-4-(furan-2-yl)-4,5-dihydrothiophene-3-carbonitrile(trans-3cf)<smiles>N#CC1=C(N)S[C@@H](C(=O)c2ccccc2)[C@H]1c1ccco1</smiles>

\section{${ }^{1} \mathrm{H}$ NMR}
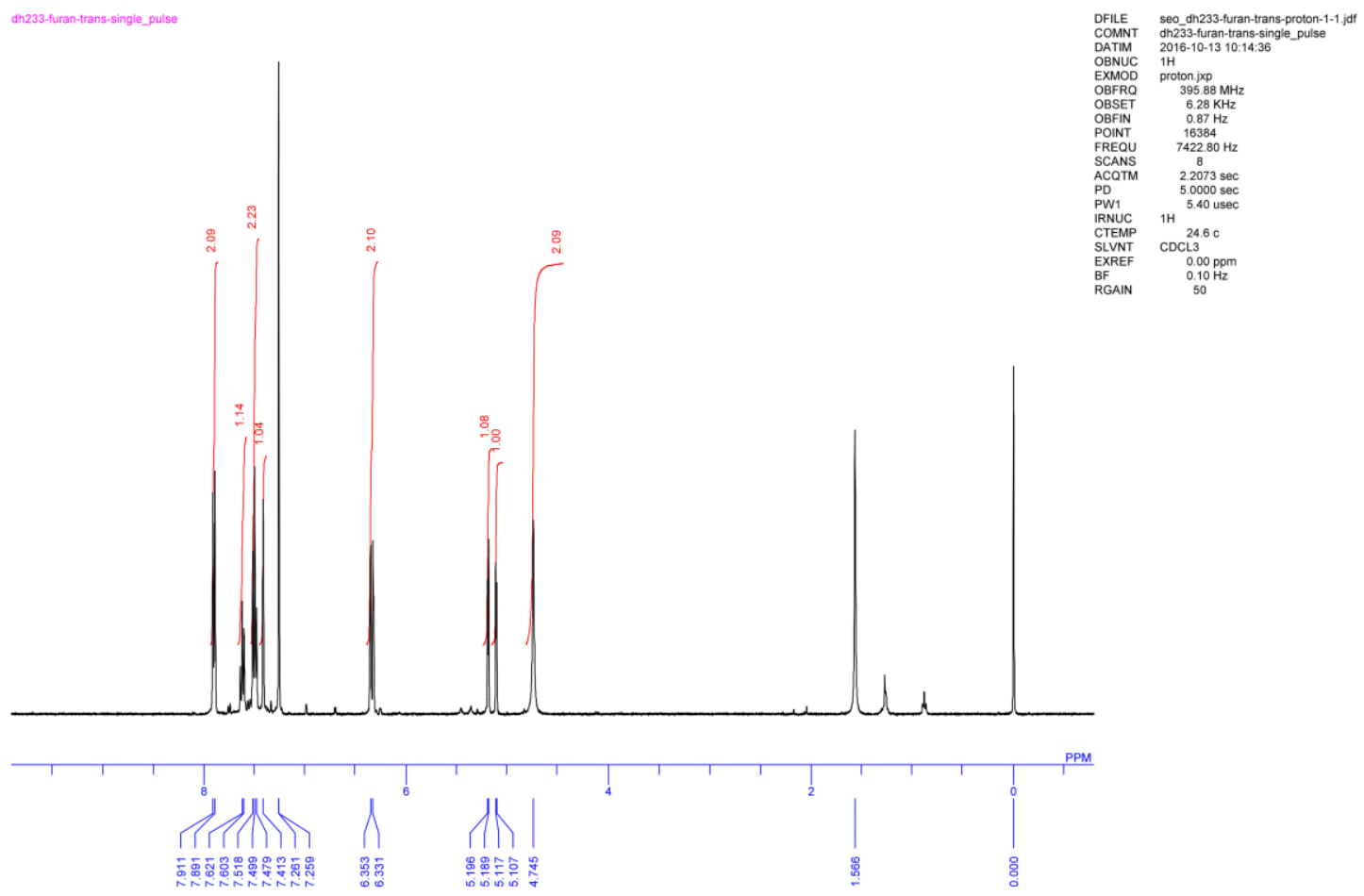
${ }^{13} \mathrm{C}$ NMR

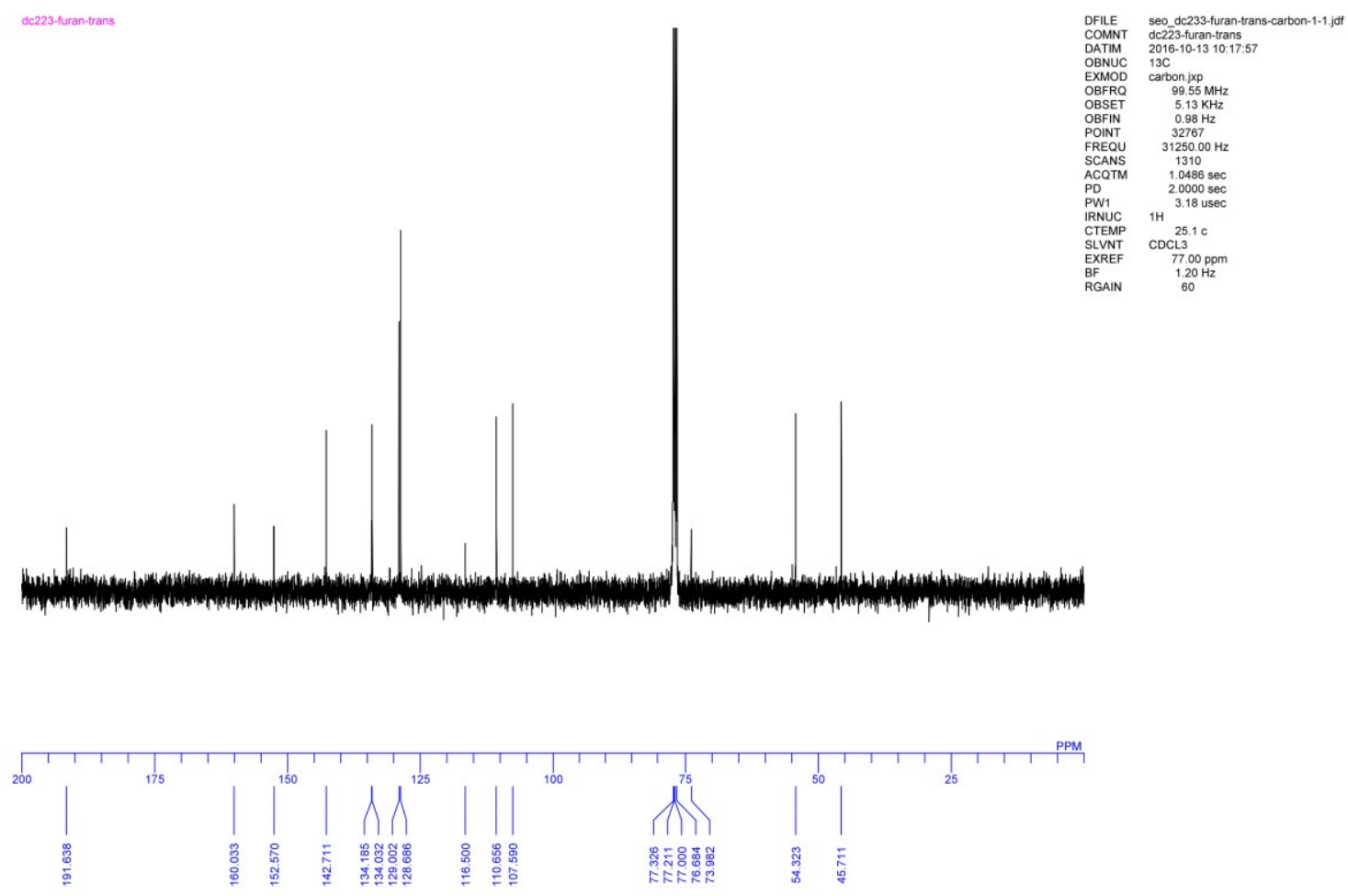

MW Profile

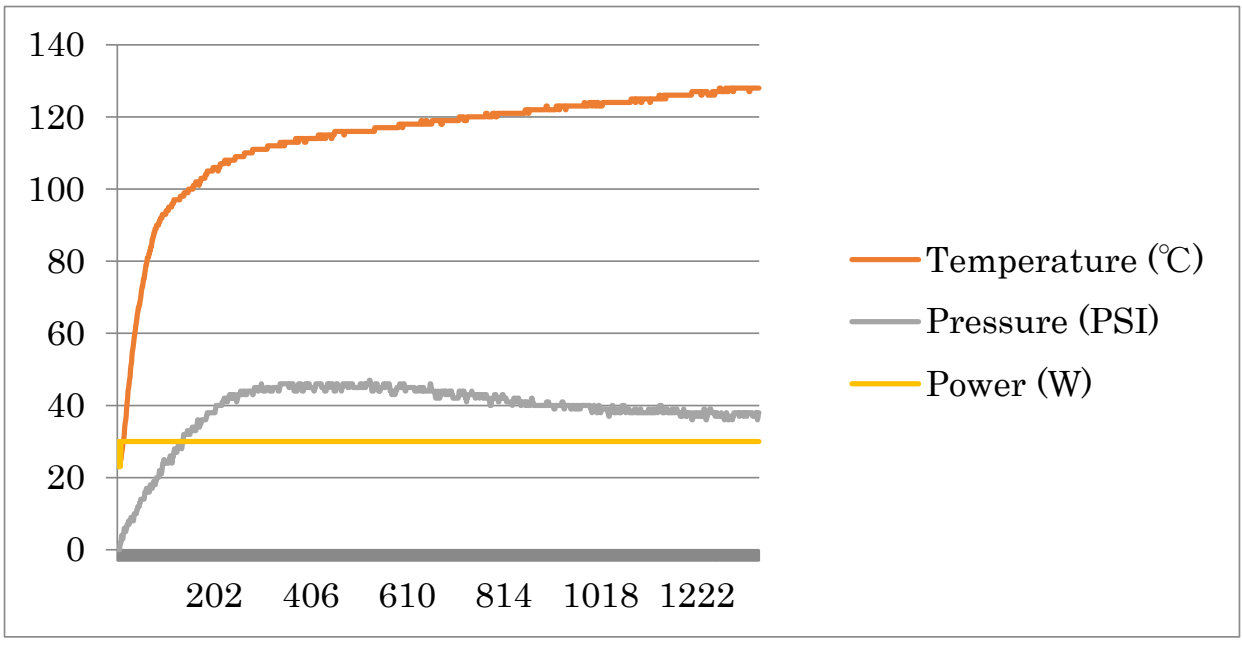


2-Amino-5-benzoyl-4-(furan-2-yl)-4,5-dihydrothiophene-3-carbonitrile (cis-3cf)<smiles>N#CC1=C(N)SC(C(=O)c2ccccc2)[C@H]1c1ccco1</smiles>

${ }^{1} \mathrm{H}$ NMR

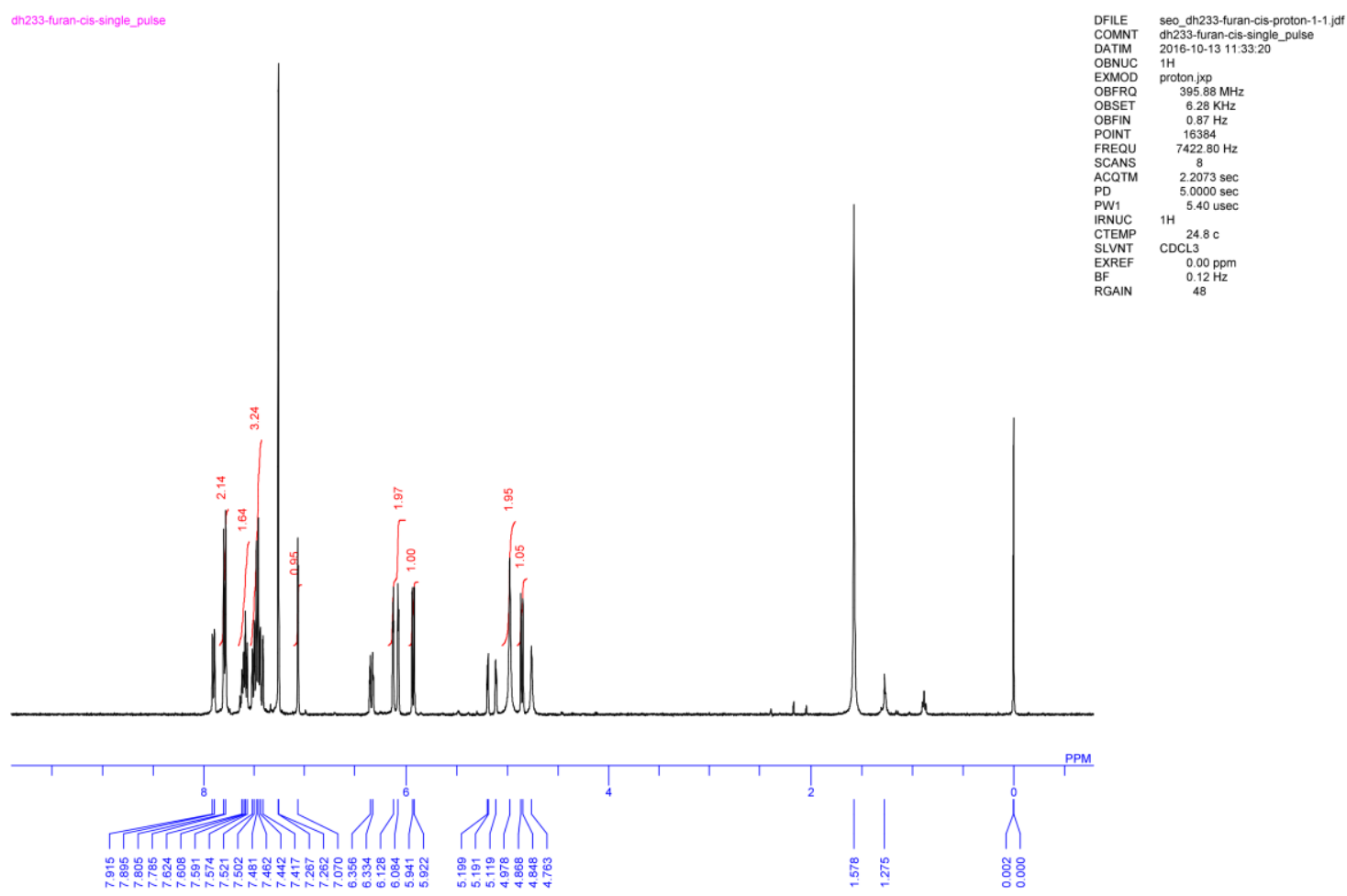


${ }^{13} \mathrm{C}$ NMR

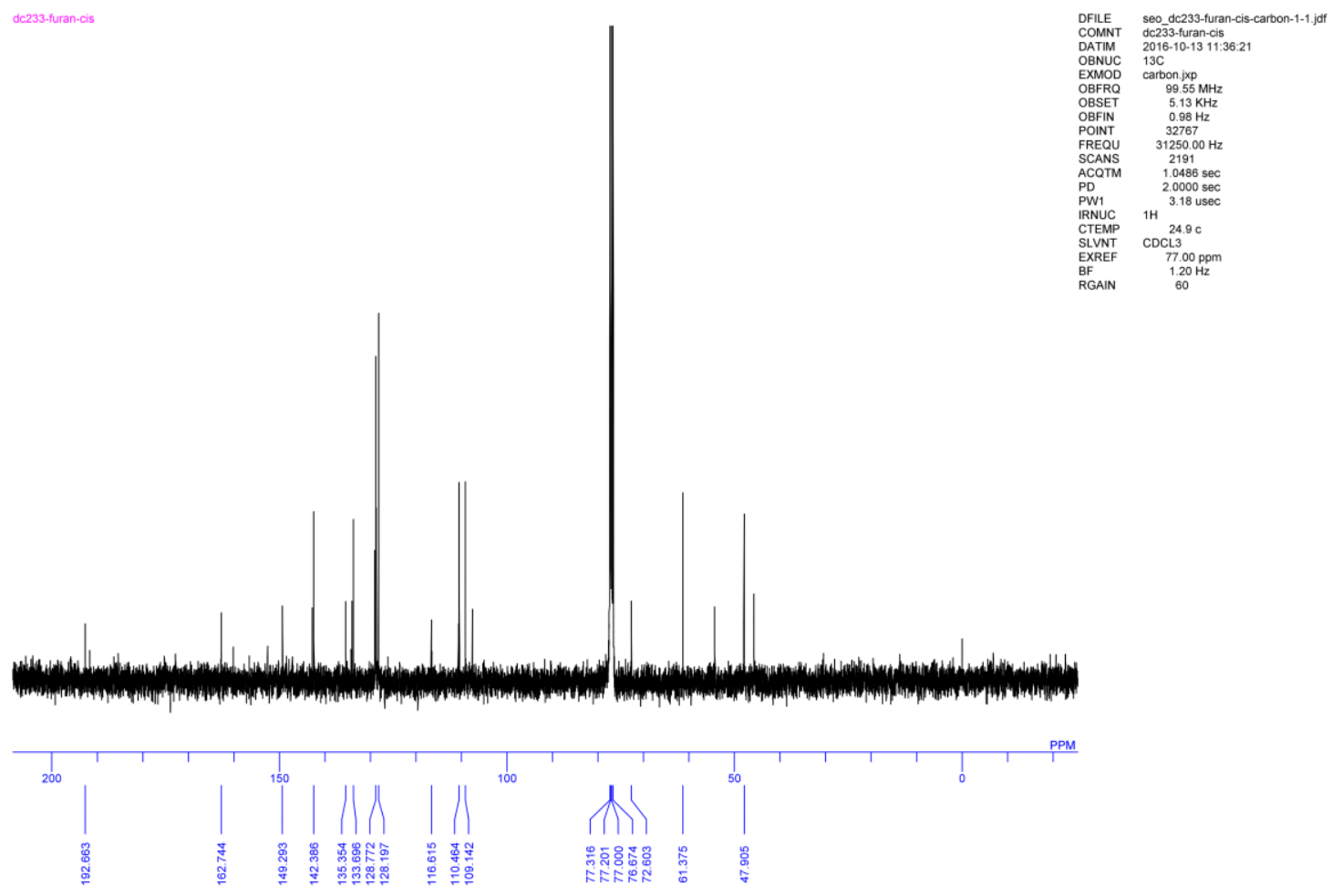


Methyl 5-acetyl-2-amino-4-phenyl-4,5-dihydrothiophene-3-carboxylate (trans-3ai)<smiles>COC(=O)C1=C(N)SC(C(C)=O)[C@H]1c1ccccc1</smiles>

${ }^{1} \mathrm{H}-\mathrm{NMR}$

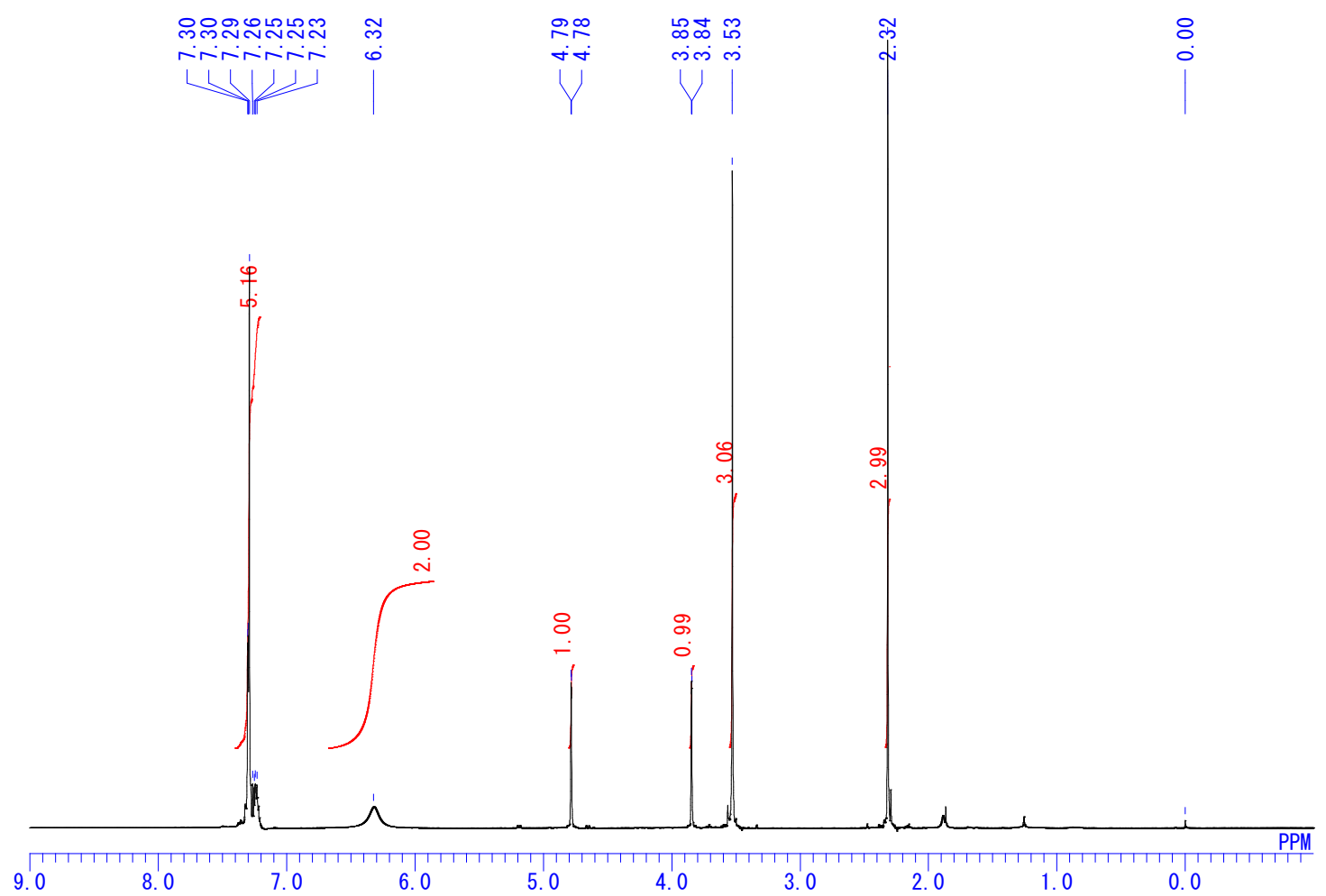


${ }^{13} \mathrm{C}-\mathrm{NMR}$

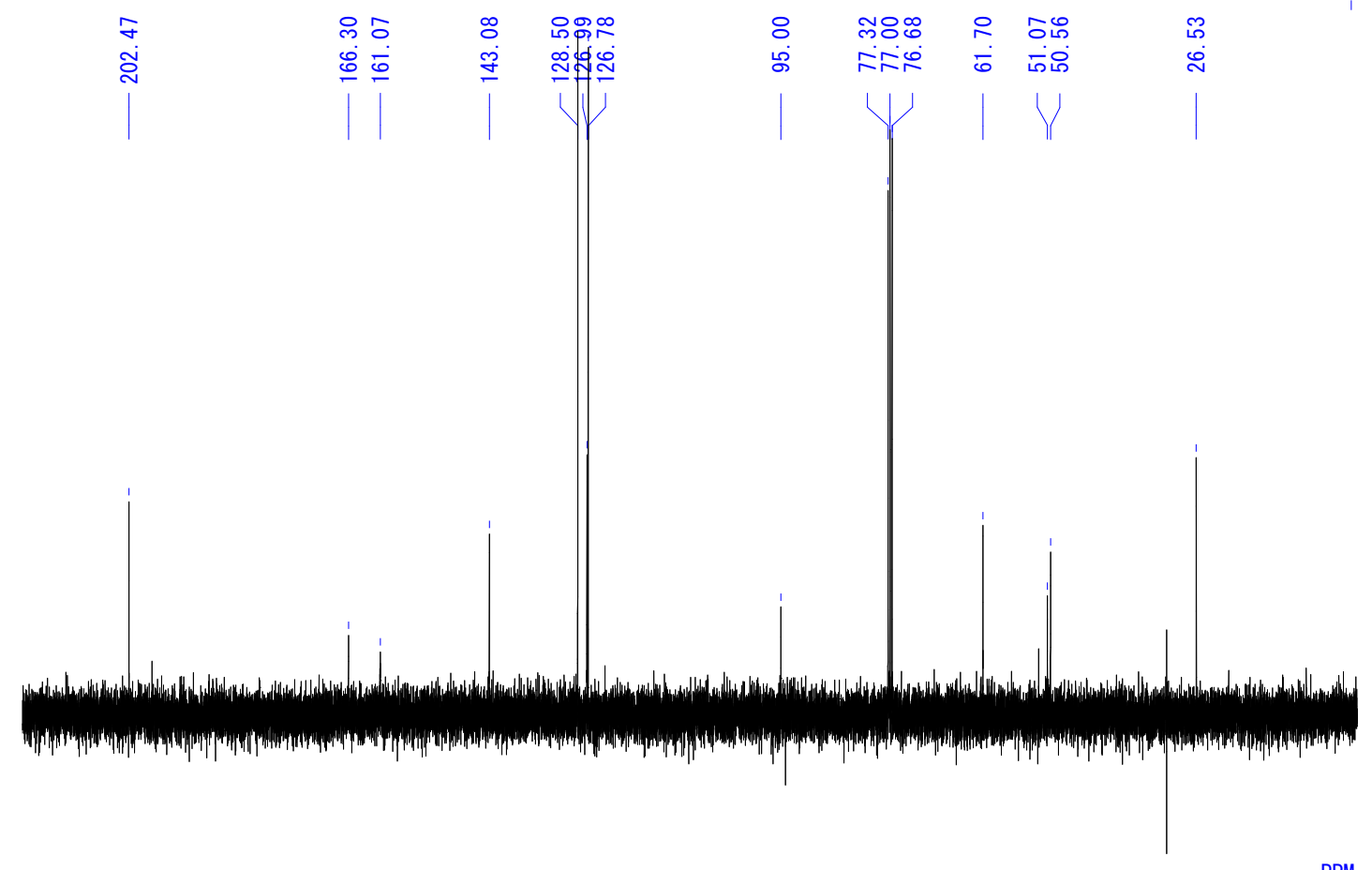

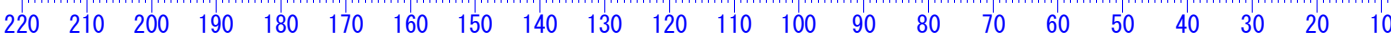

MW energy profile

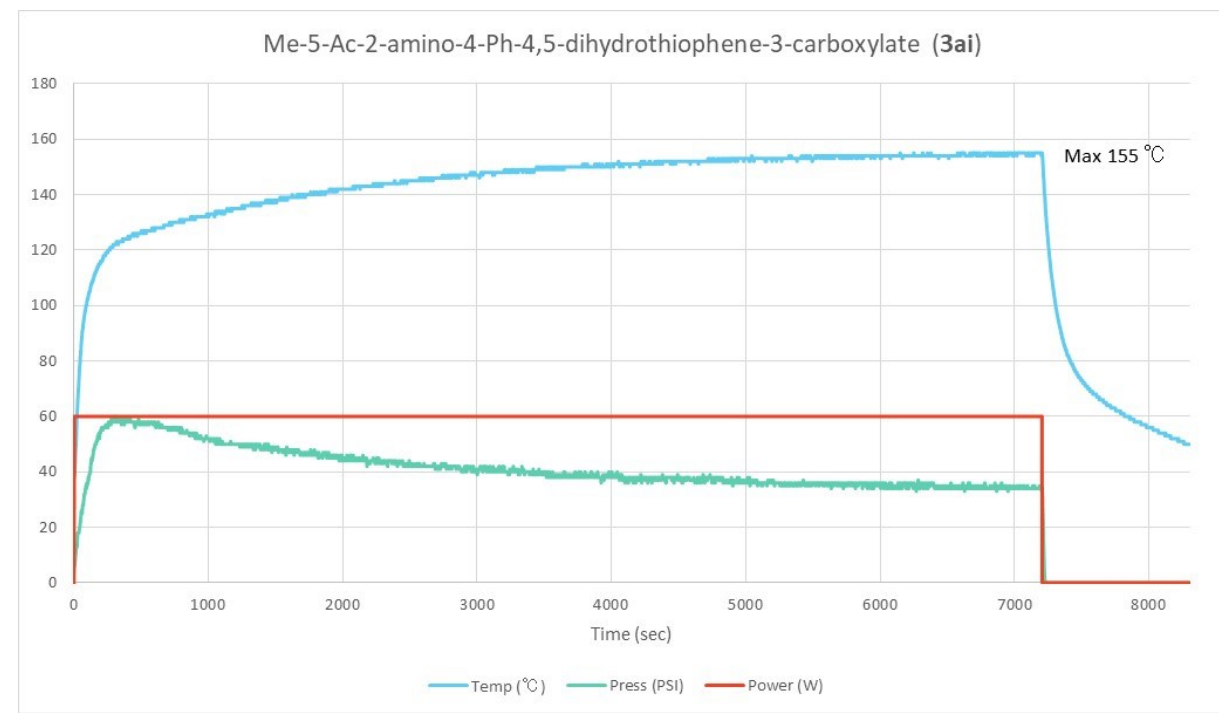


Methyl 5-acetyl-2-amino-4-phenyl-4,5-dihydrothiophene-3-carboxylate (cis-3ai)<smiles>COC(=O)C1=C(N)SC(C(C)=O)[C@H]1c1ccccc1</smiles>

${ }^{1} \mathrm{H}-\mathrm{NMR}$

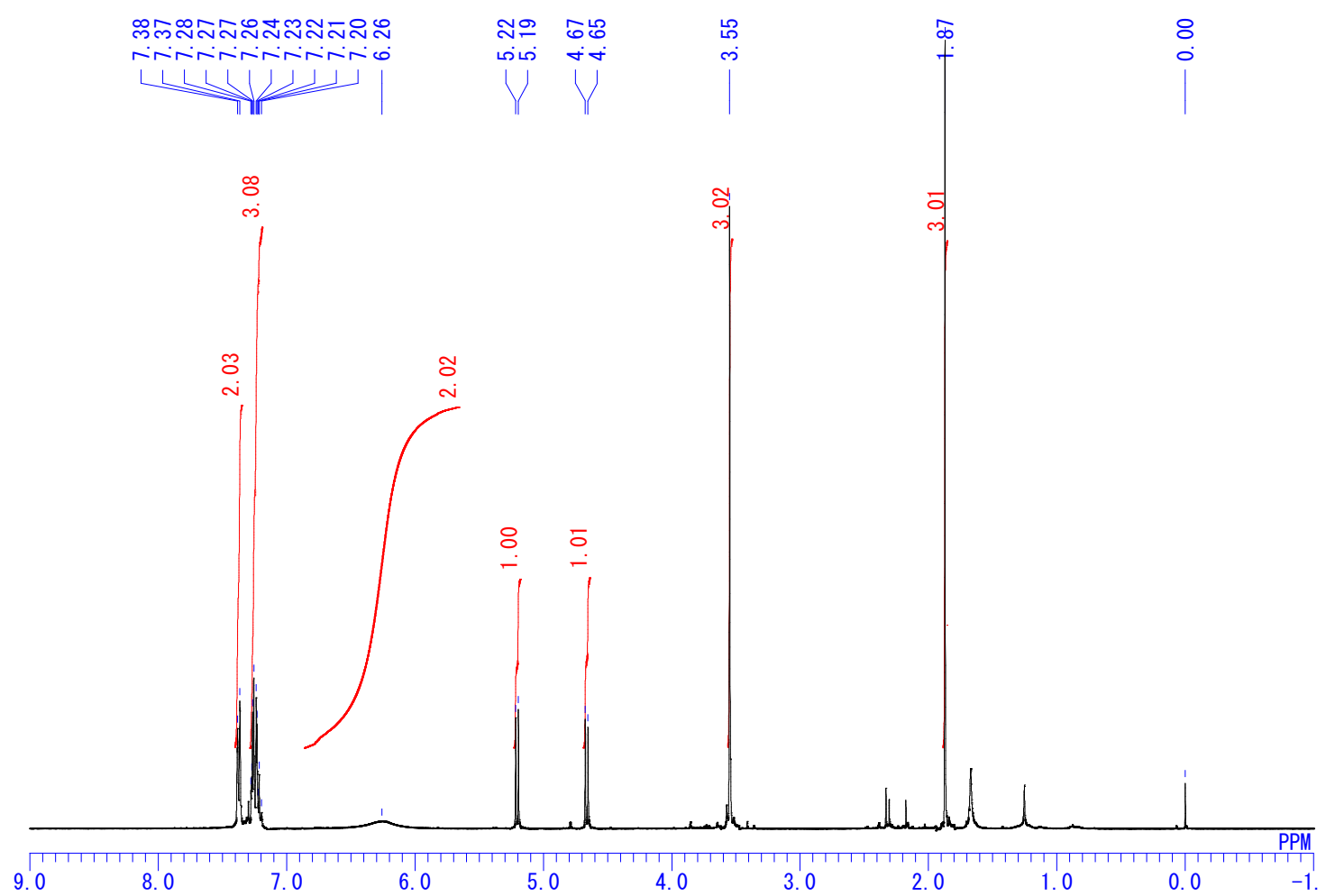


${ }^{13} \mathrm{C}-\mathrm{NMR}$

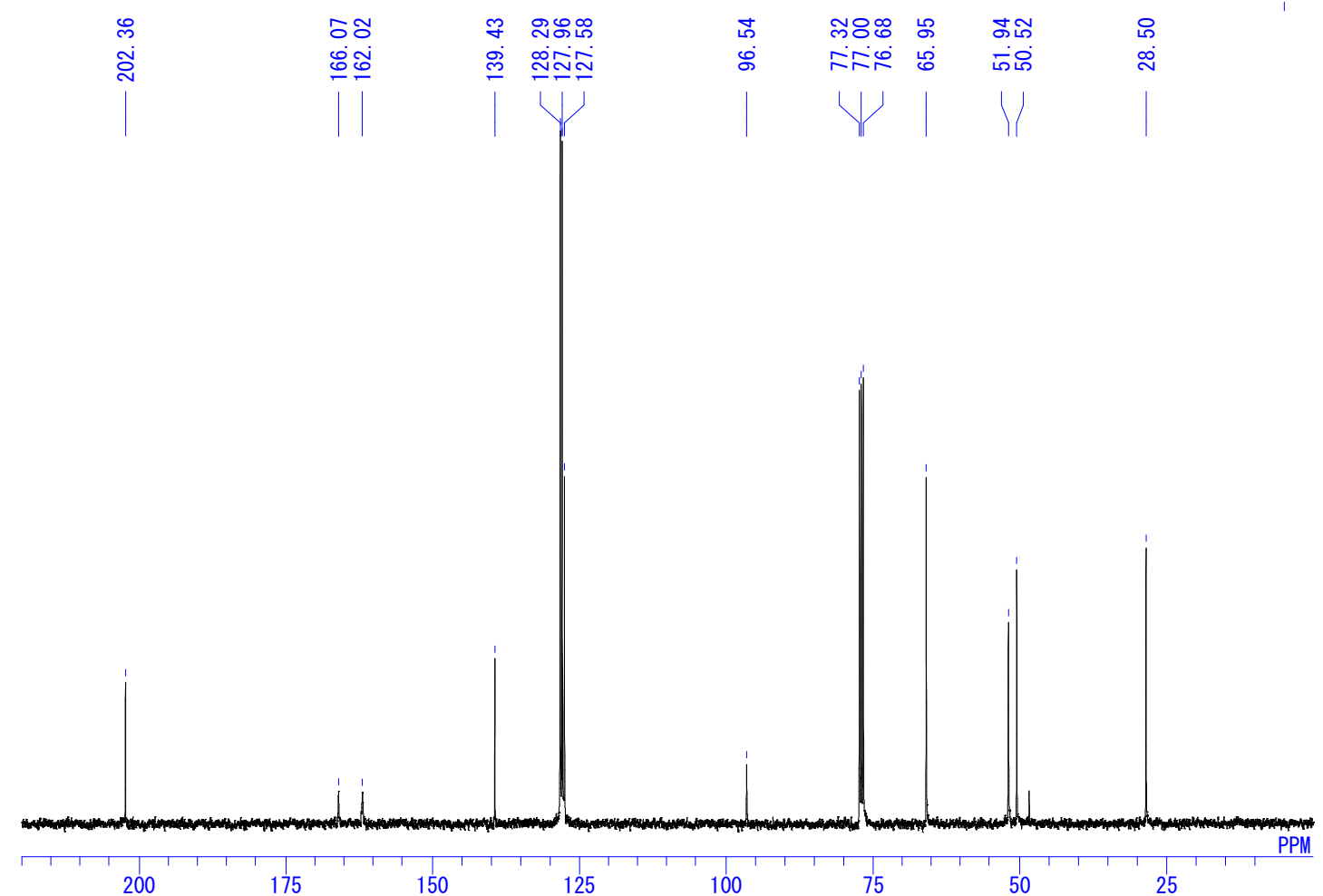


Methyl 2-amino-4-phenyl-5-pivaloyl-4,5-dihydrothiophene-3-carboxylate (trans-3bi)

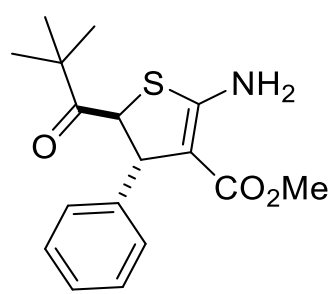

${ }^{1} \mathrm{H}-\mathrm{NMR}$

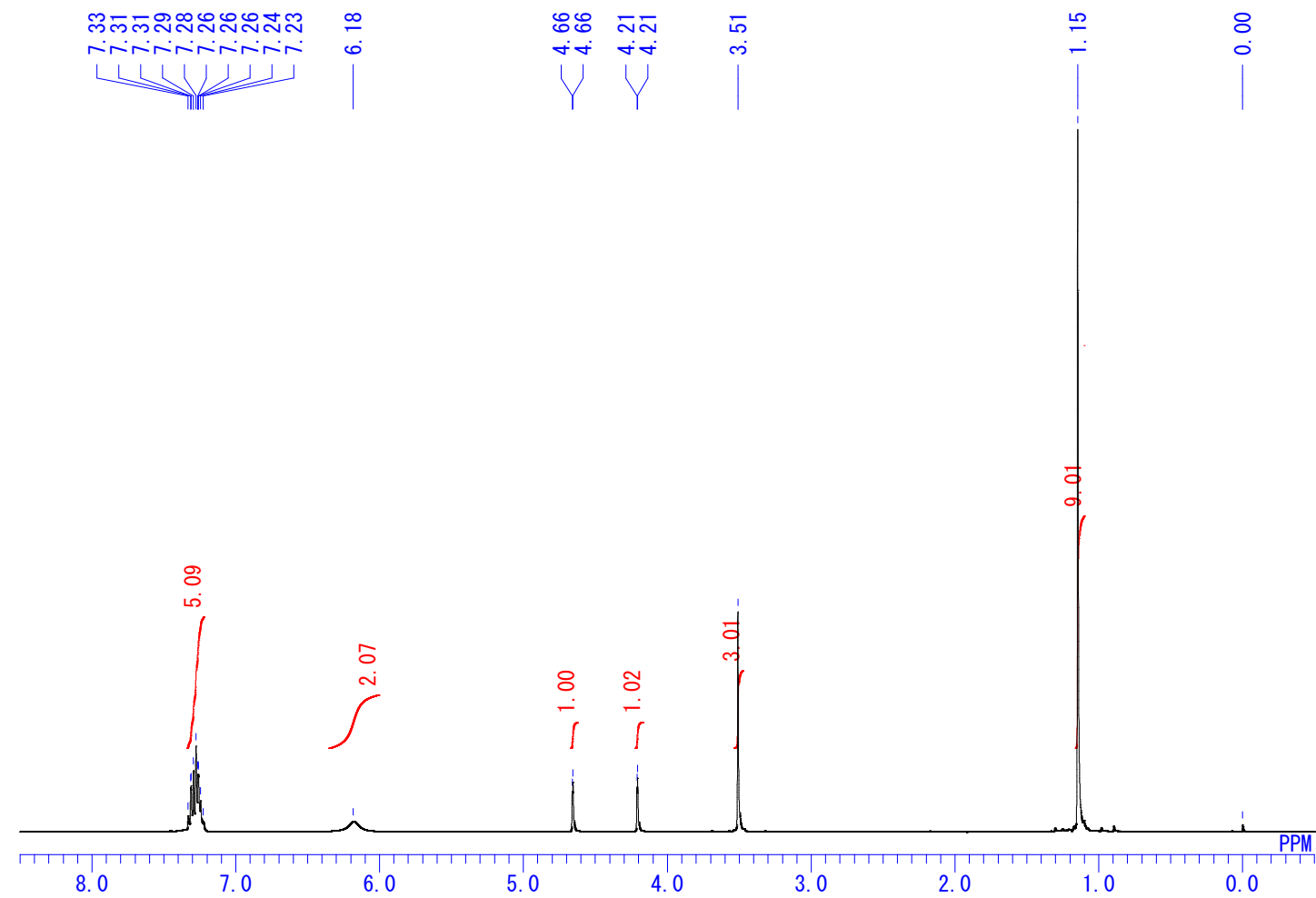


${ }^{13} \mathrm{C}-\mathrm{NMR}$

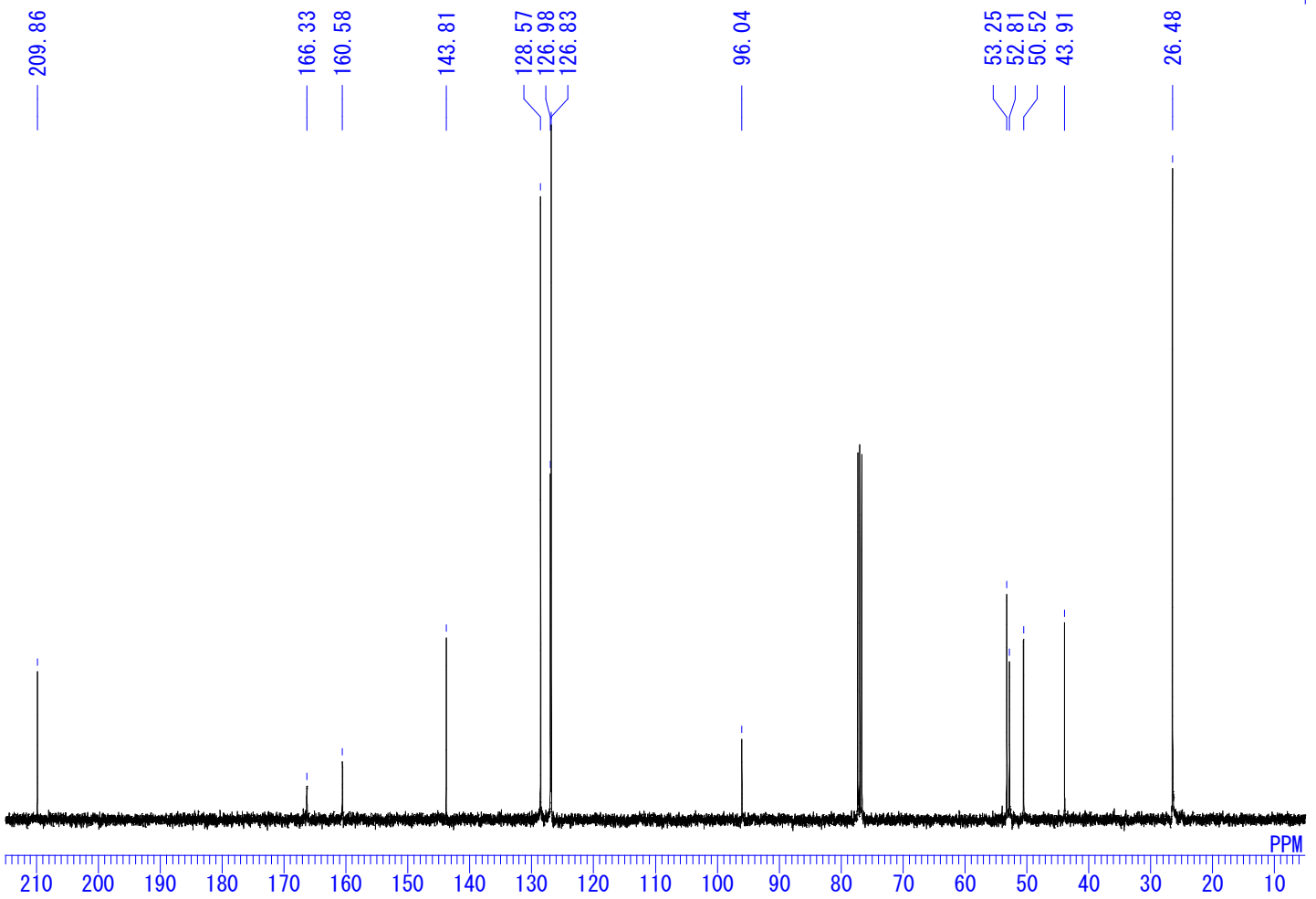

MW energy profile

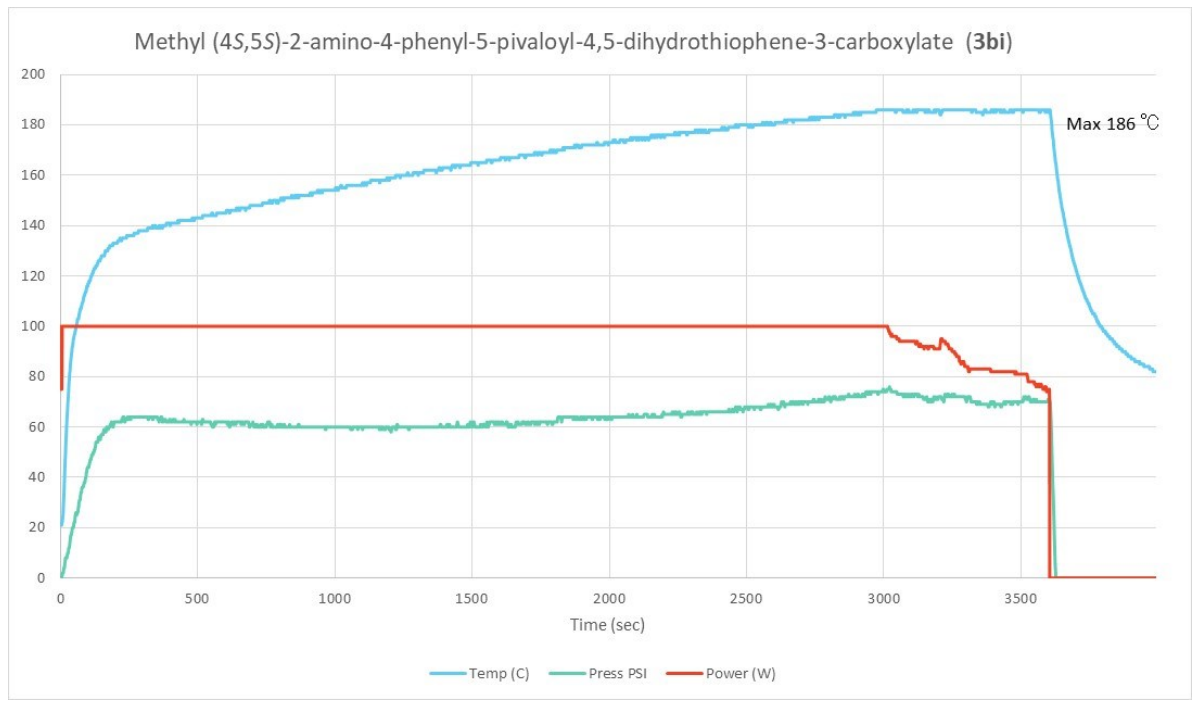


Methyl 2-amino-4-phenyl-5-pivaloyl-4,5-dihydrothiophene-3-carboxylate (cis-3bi)

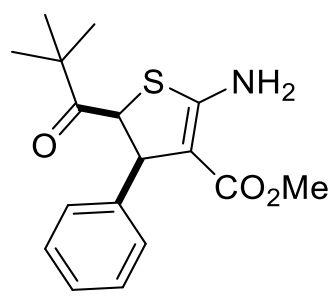

${ }^{1} \mathrm{H}-\mathrm{NMR}$

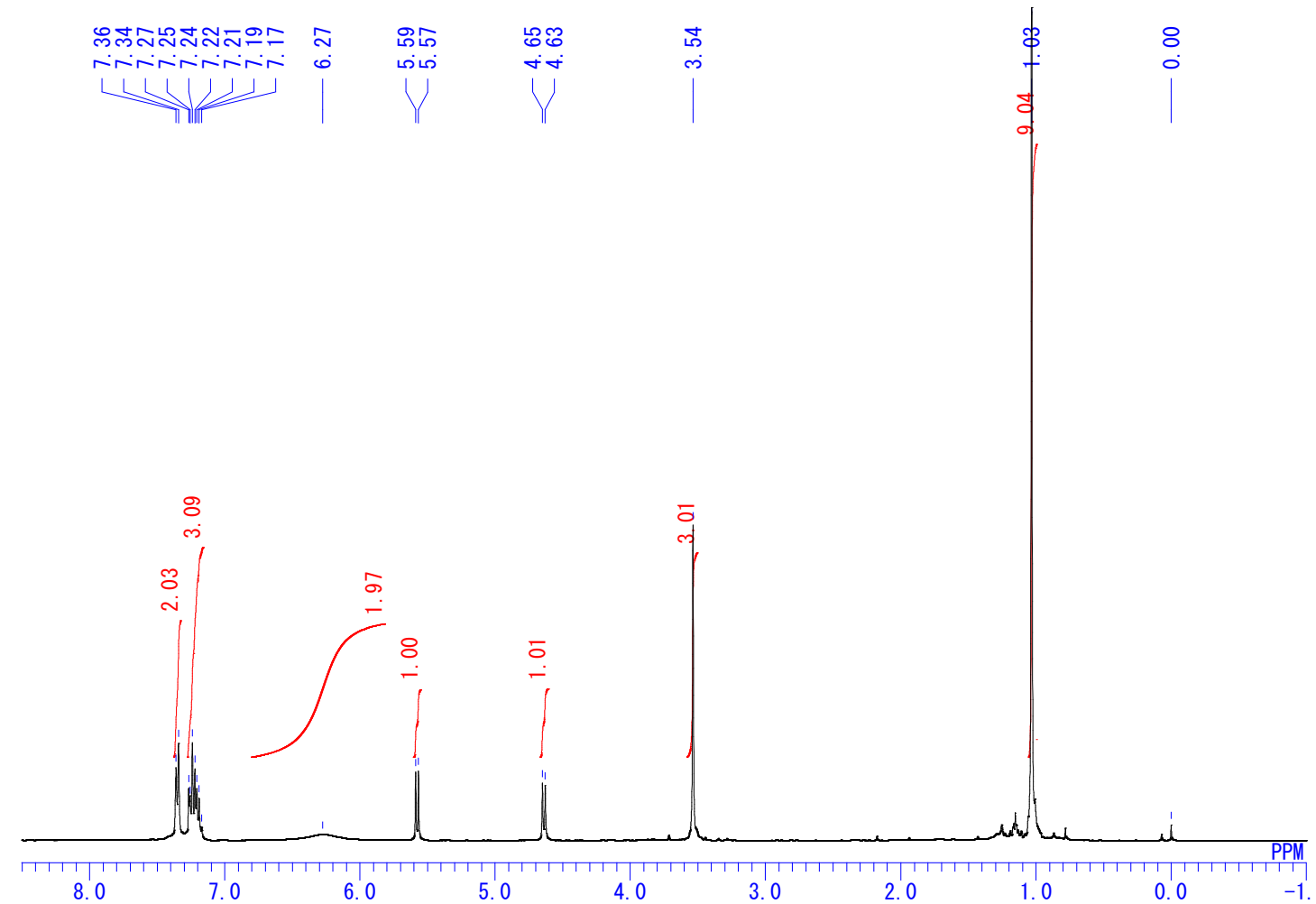


${ }^{13} \mathrm{C}-\mathrm{NMR}$

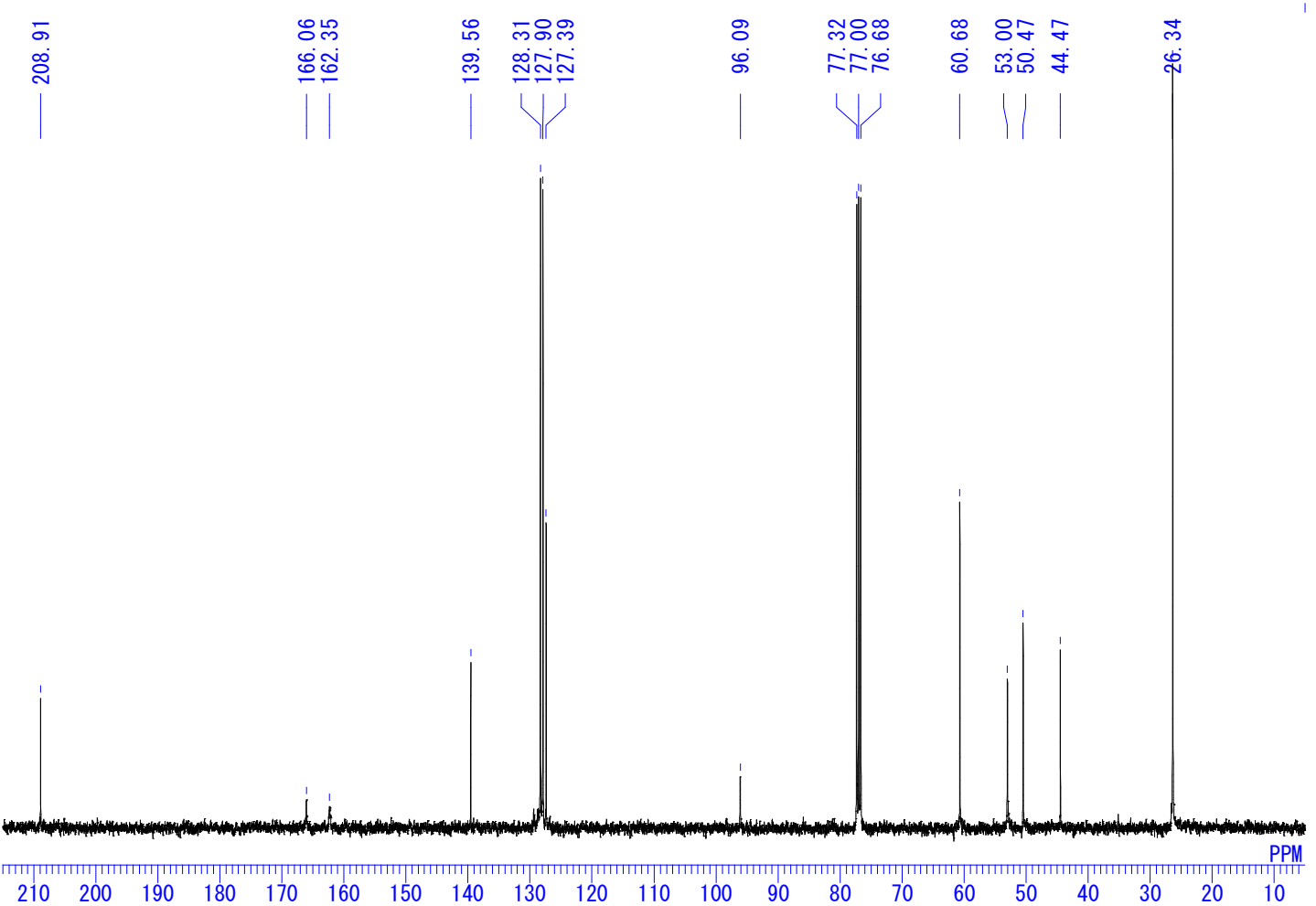


Methyl 2-amino-5-benzoyl-4-phenyl-4,5-dihydrothiophene-3-carboxylate (trans-3ci)

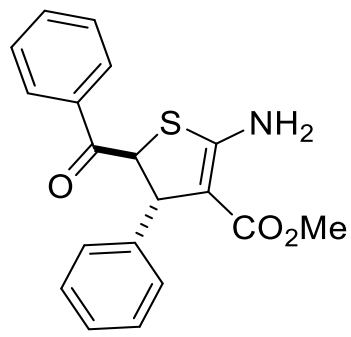

${ }^{1} \mathrm{H}-\mathrm{NMR}$

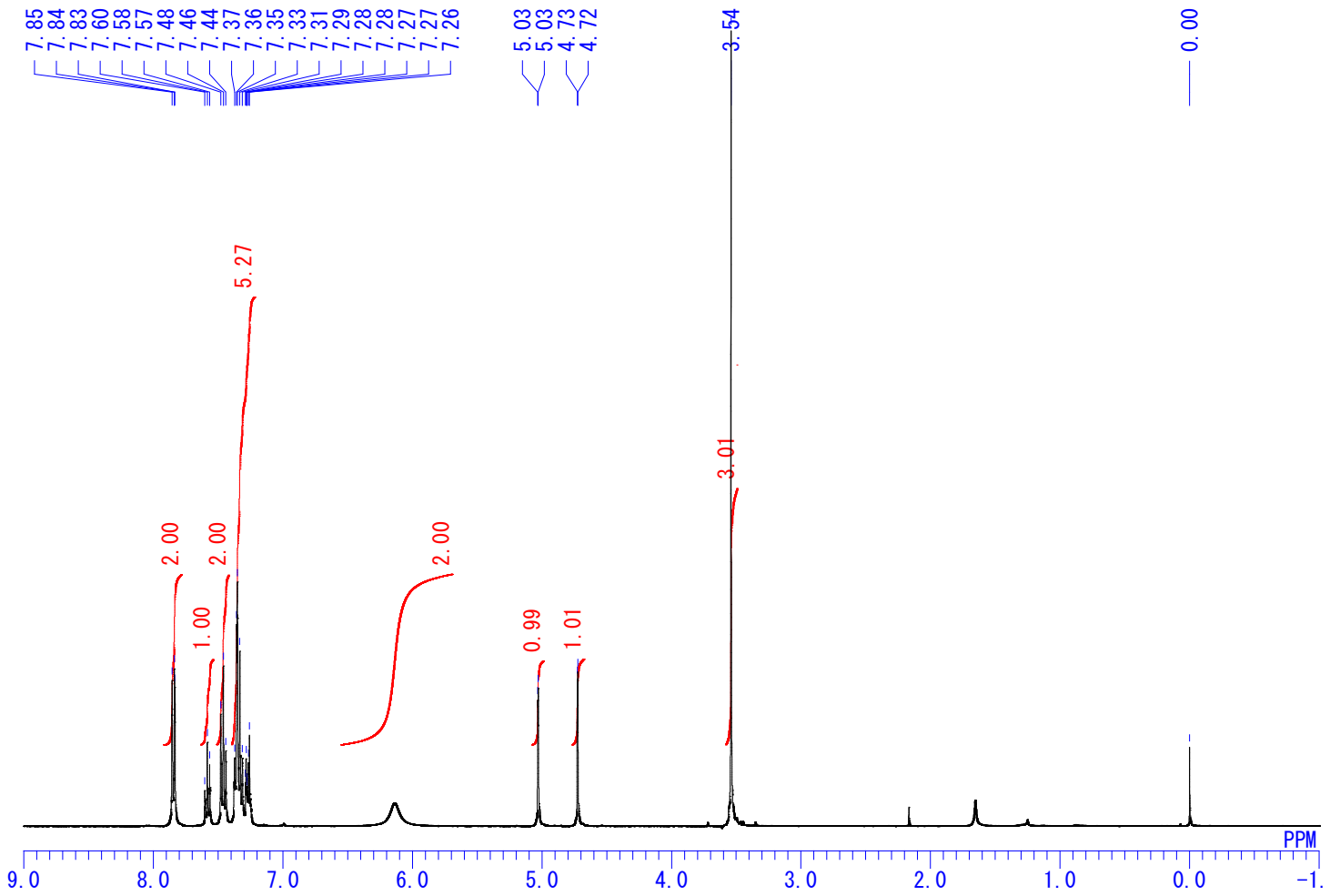


${ }^{13} \mathrm{C}-\mathrm{NMR}$

\& ₹

๙ $\quad$ \&

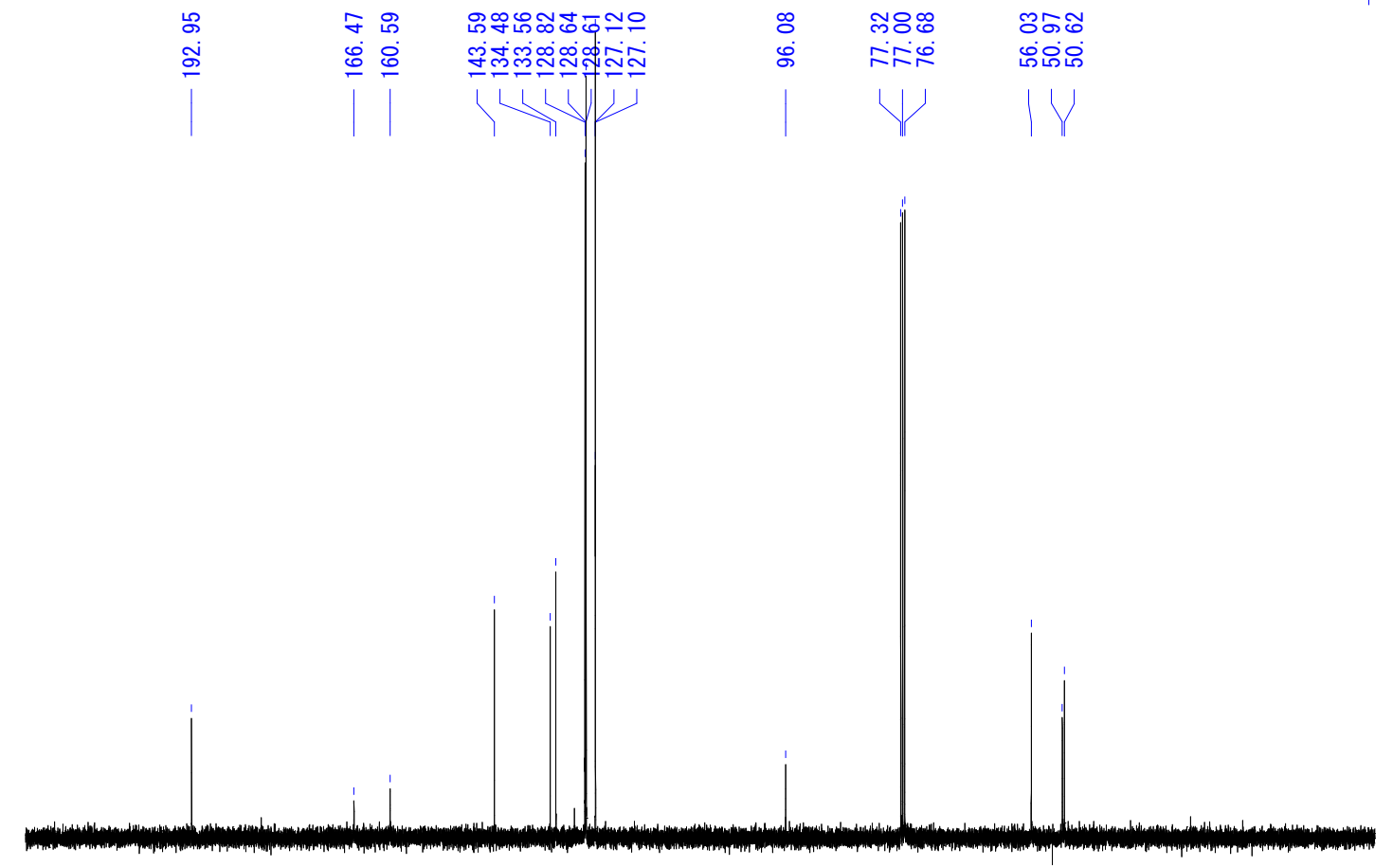

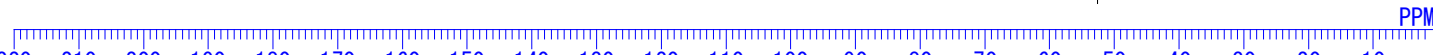
$\begin{array}{llllllllllllllllllllll}220 & 210 & 200 & 190 & 180 & 170 & 160 & 150 & 140 & 130 & 120 & 110 & 100 & 90 & 80 & 70 & 60 & 50 & 40 & 30 & 20 & 10\end{array}$

MW energy profile

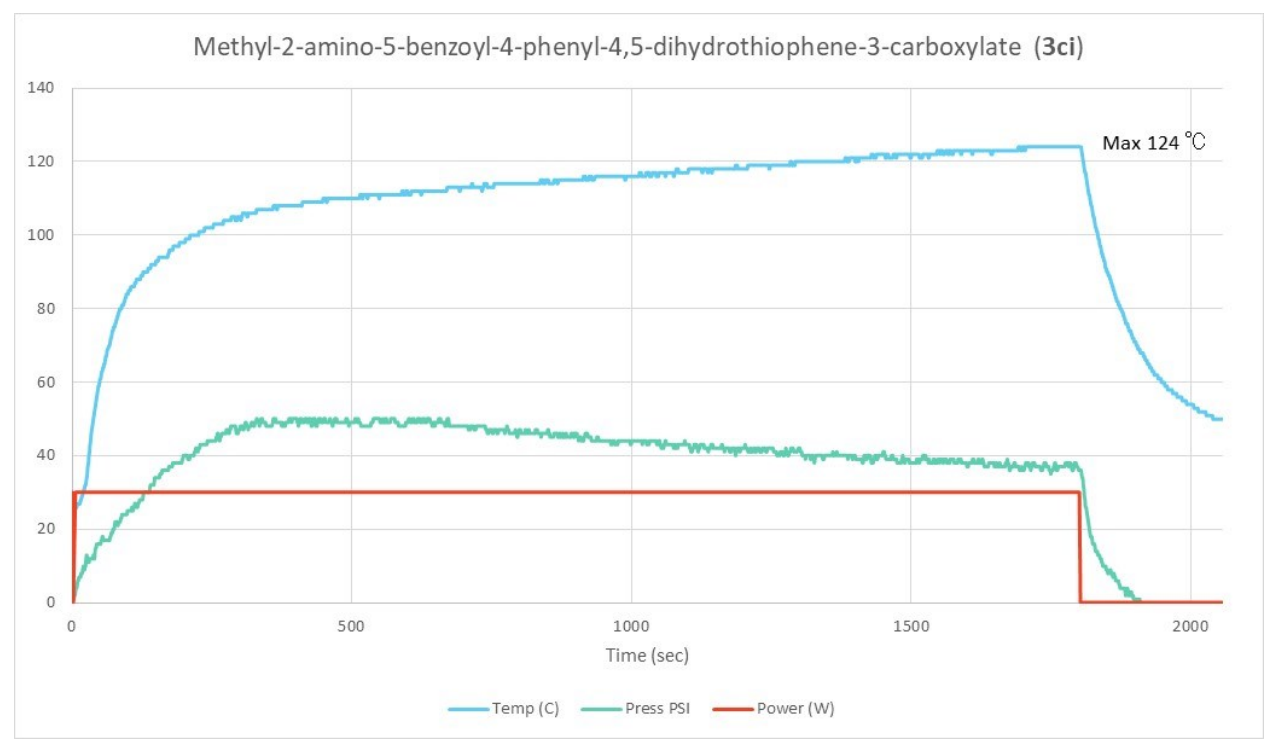


Methyl 2-amino-5-benzoyl-4-phenyl-4,5-dihydrothiophene-3-carboxylate (cis-3ci)

(CCDC; 1856497). This was recrystallized in hexane and EtOAc. ORTEP drawing is at the 50\% probability level.
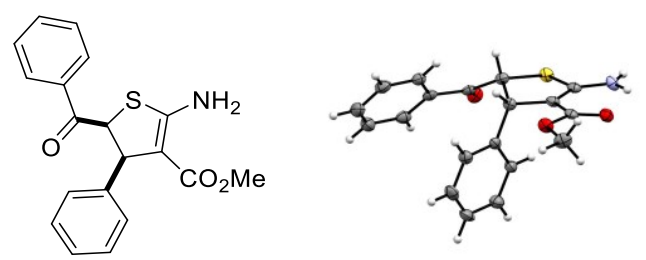

${ }^{1} \mathrm{H}-\mathrm{NMR}$

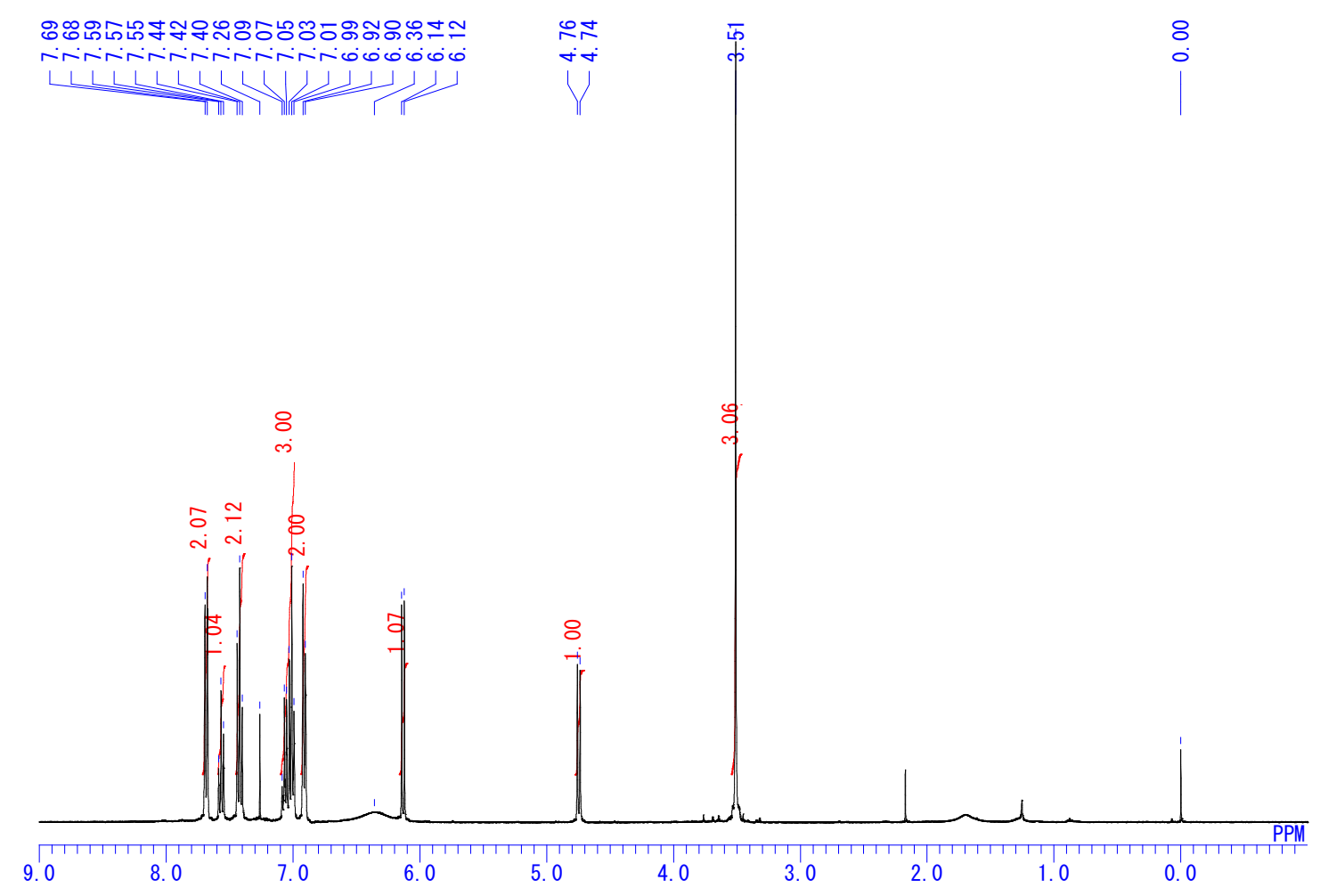


${ }^{13} \mathrm{C}$ NMR

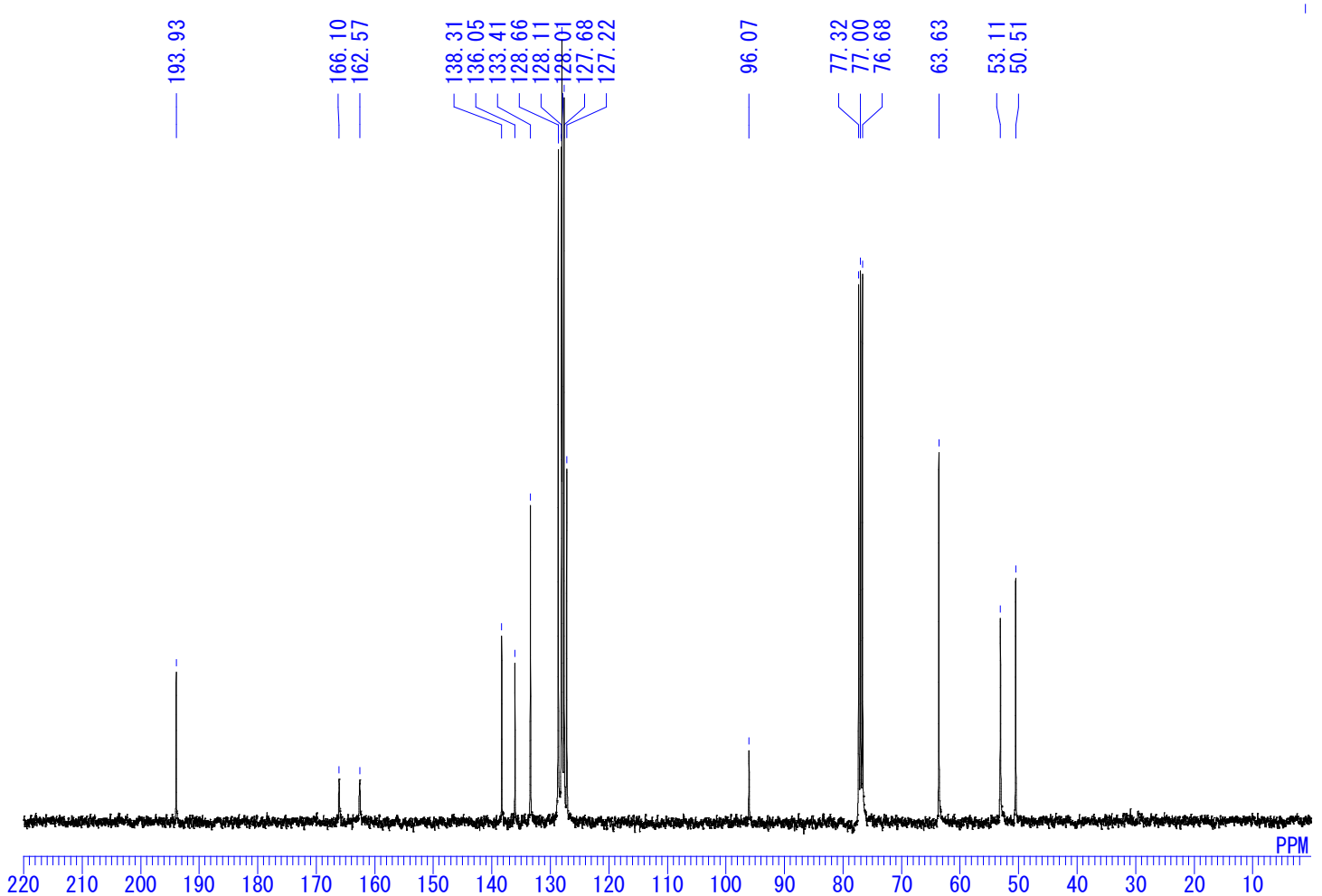


Crystal data and structure solution paramaters

\begin{tabular}{|c|c|c|c|}
\hline $\begin{array}{l}\text { Empirical } \\
\text { Formula }\end{array}$ & $\begin{array}{l}\mathrm{C}_{19} \mathrm{H}_{1} \\
{ }_{7} \mathrm{NO}_{3} \mathrm{~S}\end{array}$ & Function Minimized & $\Sigma \mathrm{w}\left(\mathrm{Fo}^{2}-\mathrm{Fc}^{2}\right)^{2}$ \\
\hline $\begin{array}{l}\text { Formula } \\
\text { Weight }\end{array}$ & 339.41 & Least Squares Weights & $\begin{array}{l}\mathrm{w}=1 /\left[\sigma^{2}\left(\mathrm{Fo}^{2}\right)+(0.0379 \cdot \mathrm{P})^{2}+0.0641 \cdot \mathrm{P}\right] \\
\text { where } \mathrm{P}=\left(\operatorname{Max}\left(\mathrm{Fo}^{2}, 0\right)+2 \mathrm{Fc}^{2}\right) / 3\end{array}$ \\
\hline $\begin{array}{l}\text { Crystal Color, } \\
\text { Habit }\end{array}$ & $\begin{array}{l}\text { metallic } \\
\text { lightcol } \\
\text { o, block }\end{array}$ & No. Observations (All reflections) & 4011 \\
\hline $\begin{array}{l}\text { Crystal } \\
\text { Dimensions }\end{array}$ & $\begin{array}{l}0.178 \mathrm{X} \\
0.140 \mathrm{X} \\
0.055 \\
\mathrm{~mm}\end{array}$ & No. Variables & 217 \\
\hline Crystal System & $\begin{array}{l}\text { orthorh } \\
\text { ombic }\end{array}$ & Reflection/Parameter Ratio & 18.48 \\
\hline \multirow[t]{7}{*}{ Lattice Type } & $\begin{array}{l}\text { Primitiv } \\
\mathrm{e}\end{array}$ & Residuals: R1 (I>2.00б(I)) & 0.0342 \\
\hline & $\begin{array}{l}a= \\
9.0236( \\
6) \AA\end{array}$ & Residuals: R (All reflections) & 0.0419 \\
\hline & $\begin{array}{l}\mathrm{b}= \\
9.2931( \\
\text { 6) } \AA\end{array}$ & Residuals: wR2 (All reflections) & 0.0784 \\
\hline & $\begin{array}{l}c= \\
20.0094 \\
(14) \AA\end{array}$ & Goodness of Fit Indicator & 1.066 \\
\hline & $\begin{array}{l}V= \\
1677.93 \\
\text { (19) } \AA^{3}\end{array}$ & Max Shift/Error in Final Cycle & 0.000 \\
\hline & & Maximum peak in Final Diff. Map & $0.22 \mathrm{e}^{-/} / \AA^{3}$ \\
\hline & & Minimum peak in Final Diff. Map & $-0.23 \mathrm{e}^{-} / \AA^{3}$ \\
\hline Space Group & $\begin{array}{l}\mathrm{P} 2{ }_{1}{ }^{2}{ }^{2} \\
1(\# 19)\end{array}$ & & \\
\hline
\end{tabular}




\begin{tabular}{|l|l|l|l|}
\hline$Z$ value & 4 & & \\
\hline$D_{\text {cal }}$ & $\begin{array}{l}1.343 \\
\mathrm{~g} / \mathrm{cm}^{3}\end{array}$ & & \\
\hline$F_{000}$ & 712.00 & & \\
\hline$\mu(\mathrm{CuK} \alpha)$ & $\begin{array}{l}2.092 \\
\mathrm{~cm}^{-1}\end{array}$ & & \\
\hline Temperature & $\begin{array}{l}-150.0 \\
{ }^{\circ} \mathrm{C}\end{array}$ & & \\
\hline
\end{tabular}


4-Hydroxy-4-methyl-2-phenyldihydrothiophene-3,3(2H)-dicarbonitrile (4aa)<smiles>CC1(O)CS[C@@H](c2ccccc2)C1(C)C#N</smiles>

${ }^{1} \mathrm{H}$ NMR
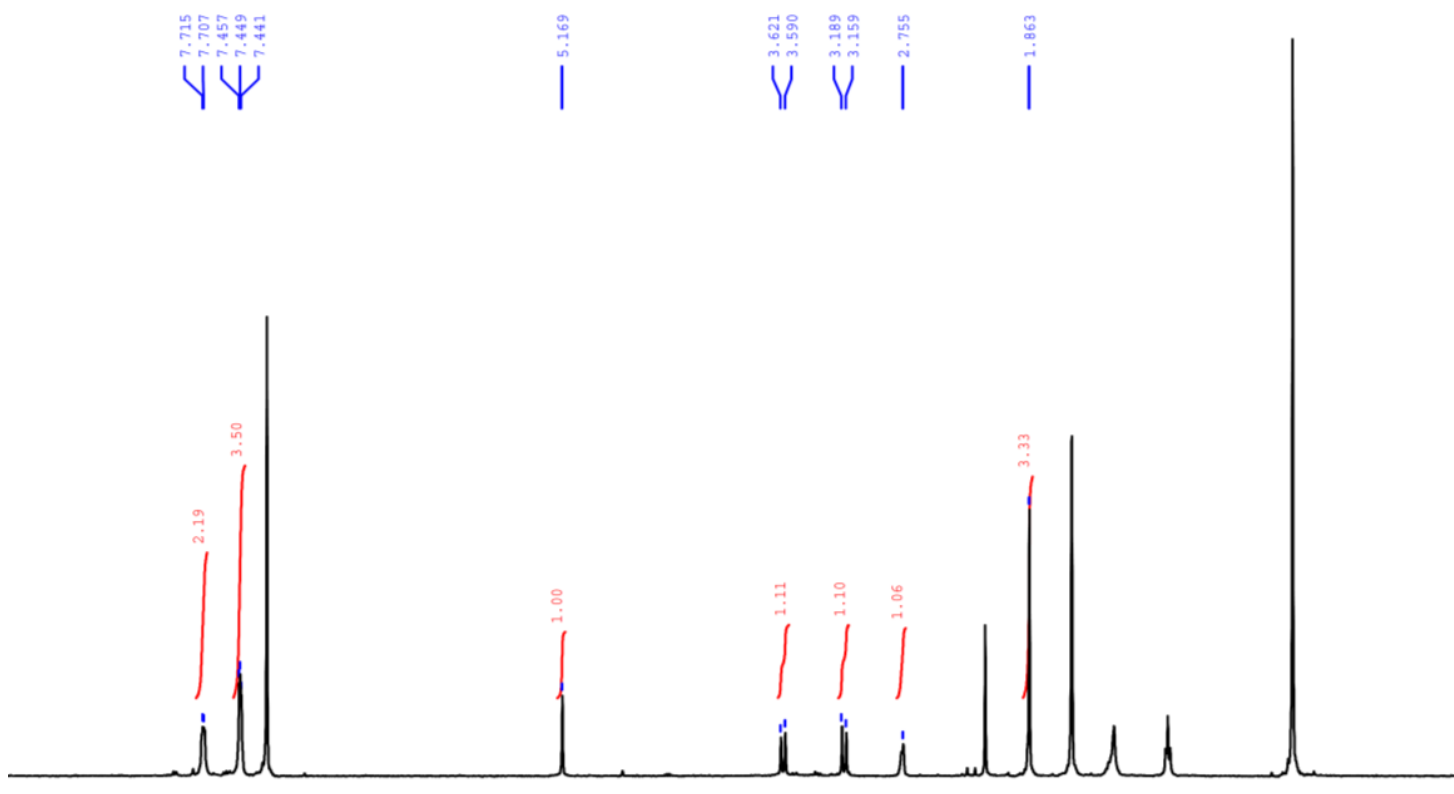

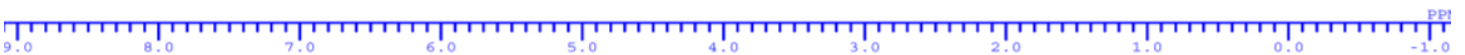


${ }^{13} \mathrm{CNMR}$

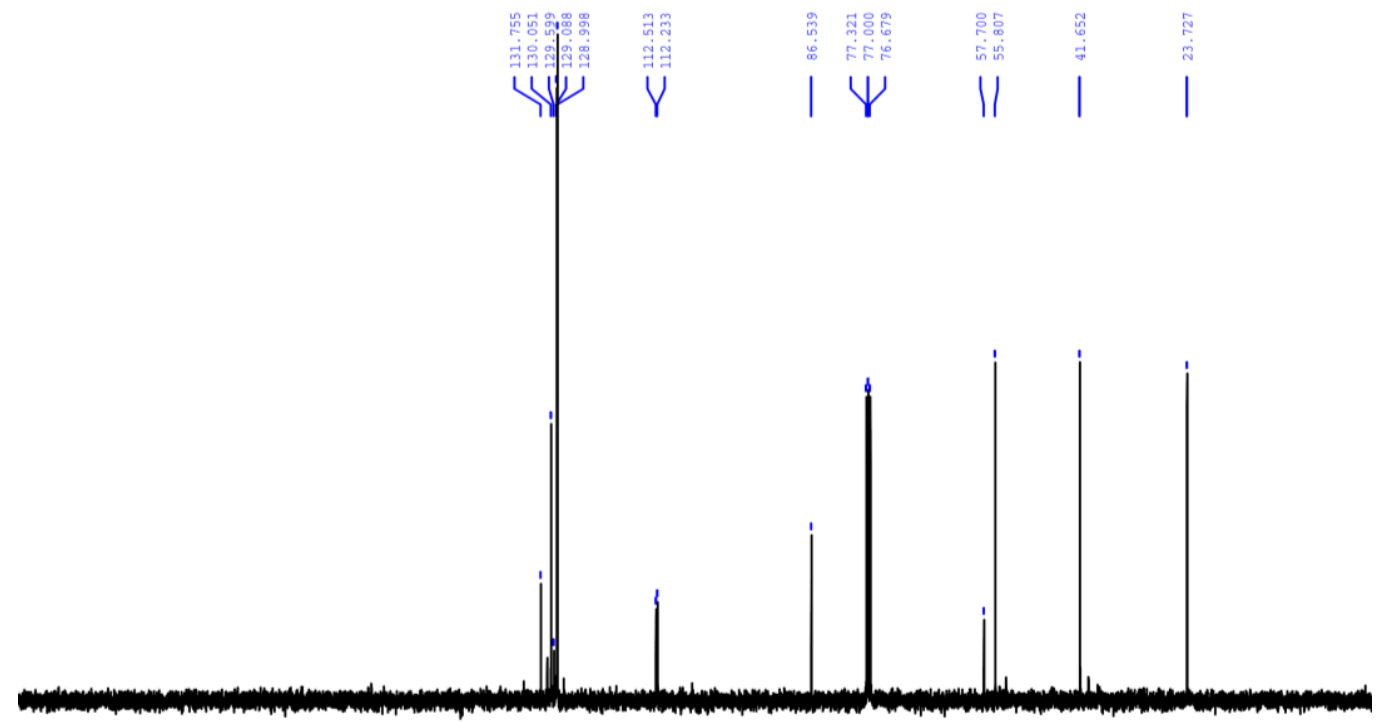

I NOESY

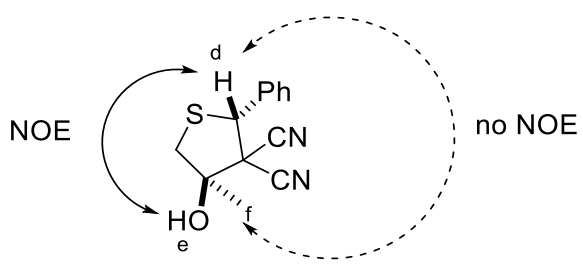

(Chemical shift of $\mathrm{O} \underline{H}$ was $\delta 3.22$ when the NOESY was observed.)

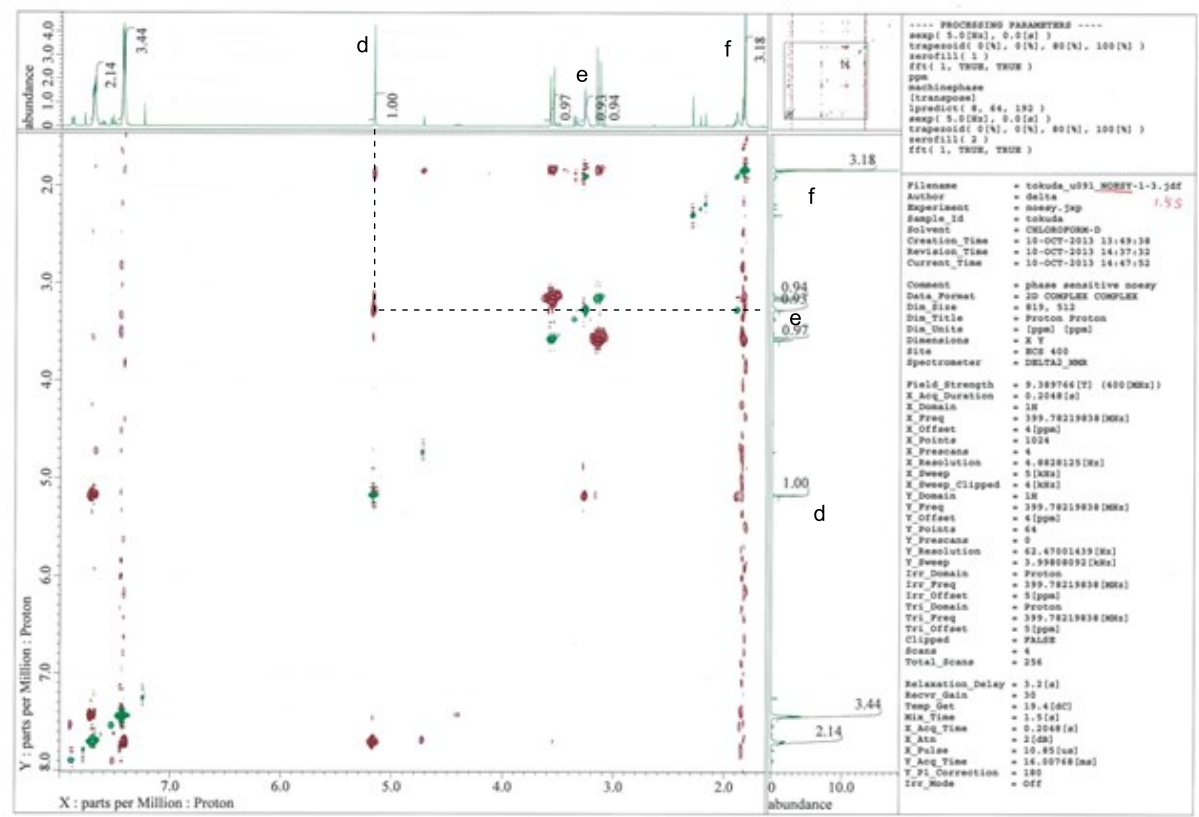


4-Hydroxy-4-methyl-2-phenyldihydrothiophene-3,3(2H)-dicarbonitrile (4ab)<smiles>C[C@]1(O)CS[C@@H](c2ccc(Cl)cc2)C1(C#N)C#N</smiles>

${ }^{1} \mathrm{H}$ NMR

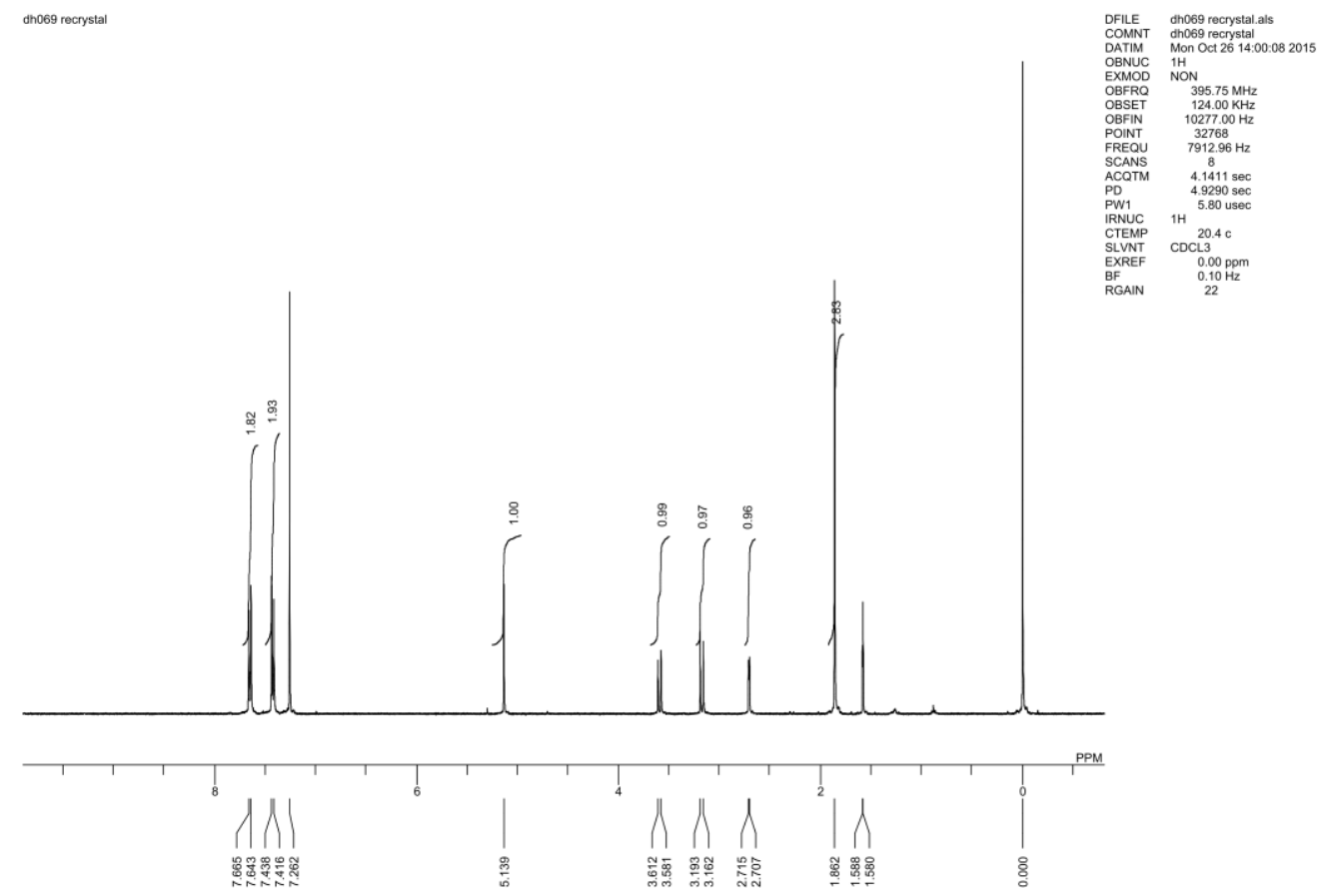


${ }^{13} \mathrm{C}$ NMR

doose

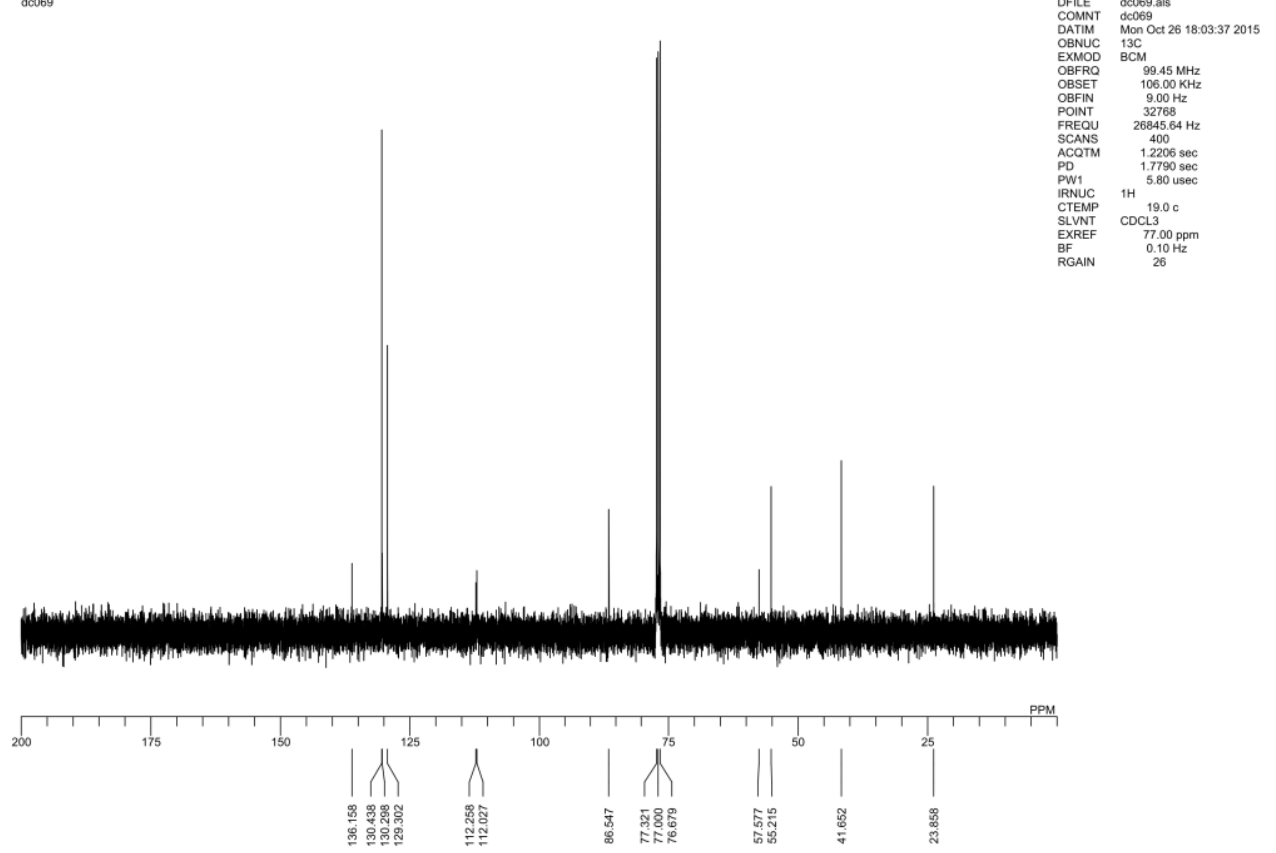


4-Hydroxy-4-methyl-2-(p-tolyl)dihydrothiophene-3,3(2H)-dicarbonitrile (4ac)<smiles>Cc1ccc([C@@H]2SC[C@@](C)(O)C2(C)C#N)cc1</smiles>

${ }^{1} \mathrm{H}$ NMR

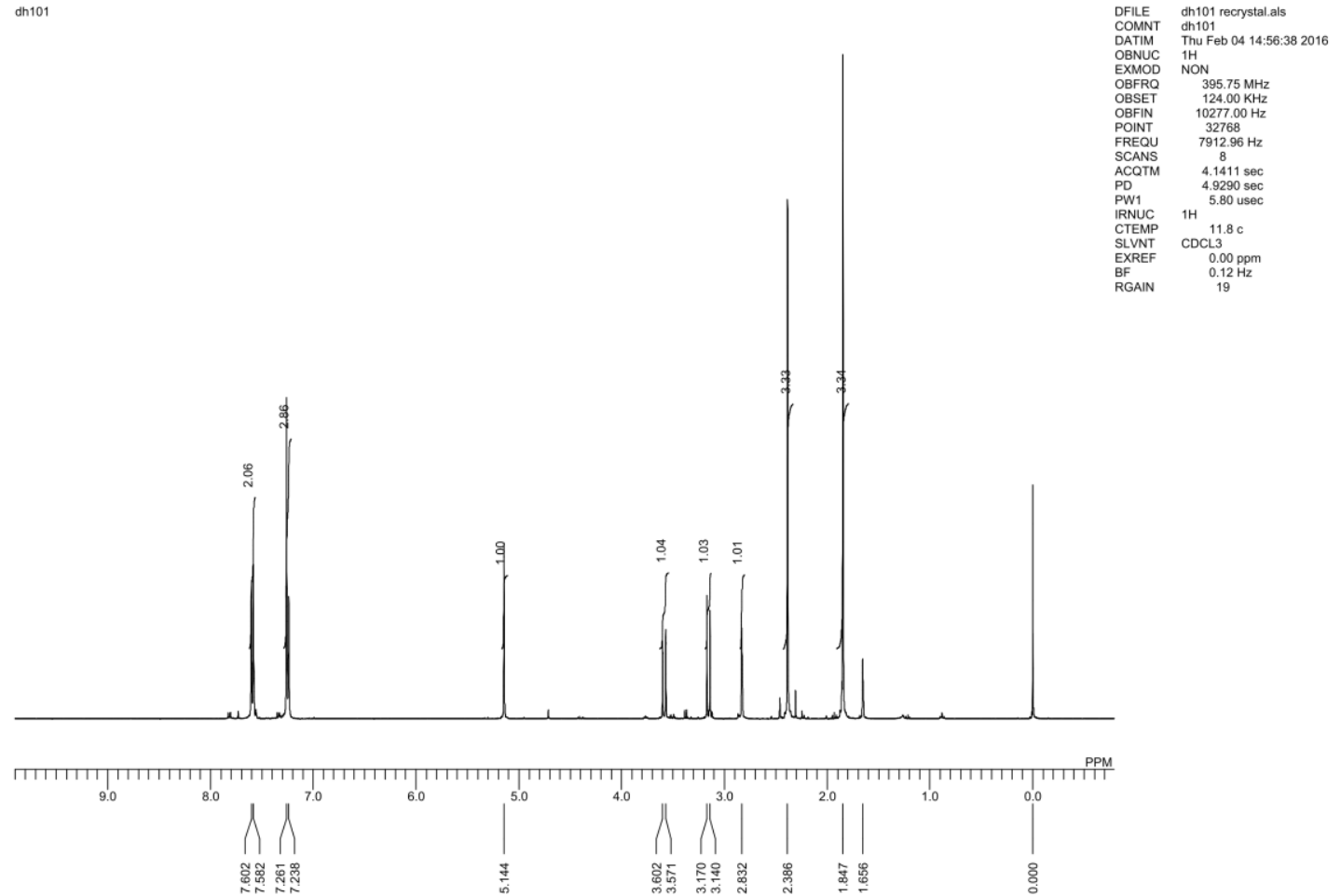


${ }^{13} \mathrm{C}$ NMR

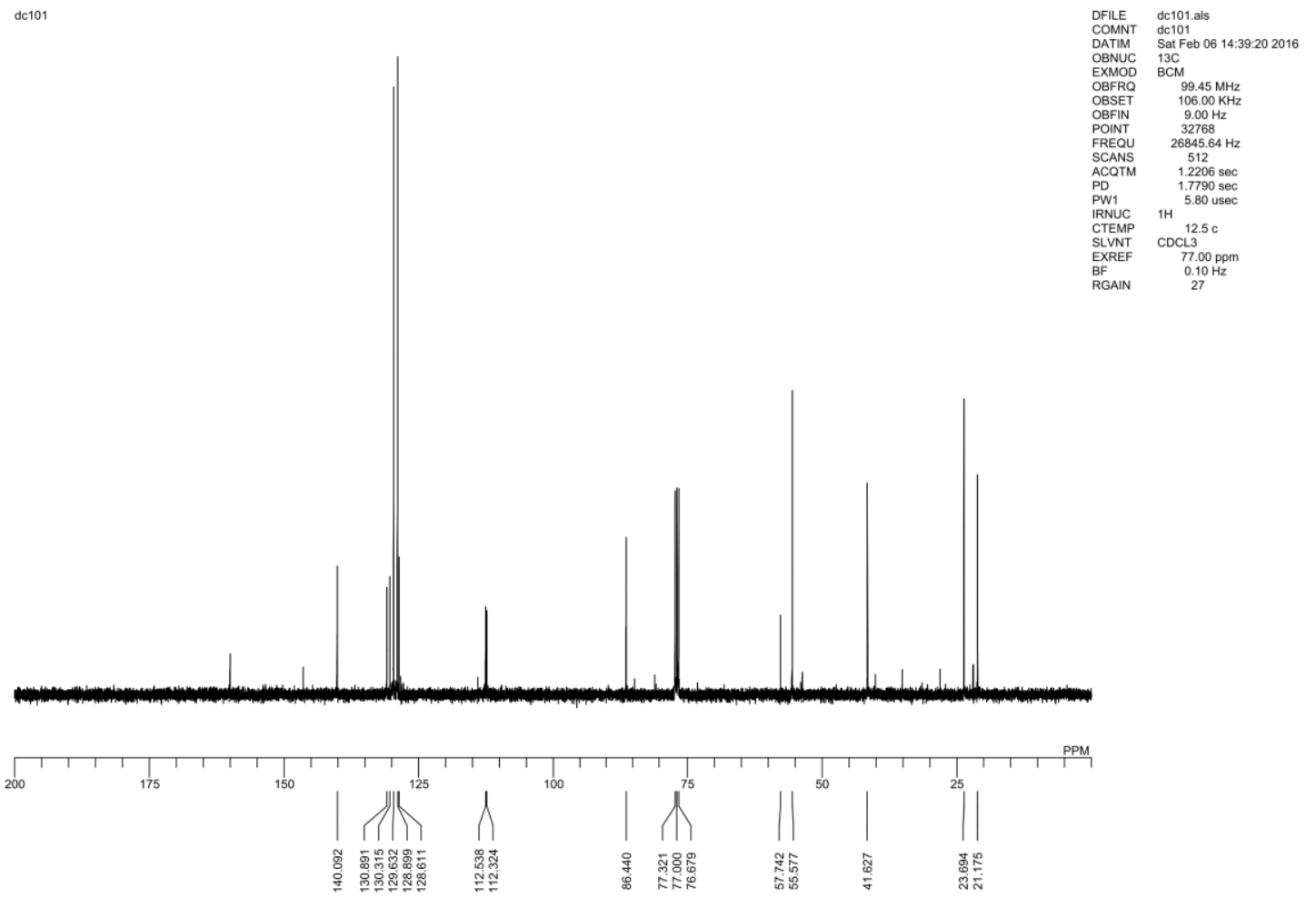


4-Hydroxy-2-(4-methoxyphenyl)-4-methyldihydrothiophene-3,3(2H)-dicarbonitrile (4ad)<smiles>COc1ccc([C@H]2SC[C@@](C)(O)C2(C)C#N)cc1</smiles>

${ }^{1} \mathrm{H}$ NMR

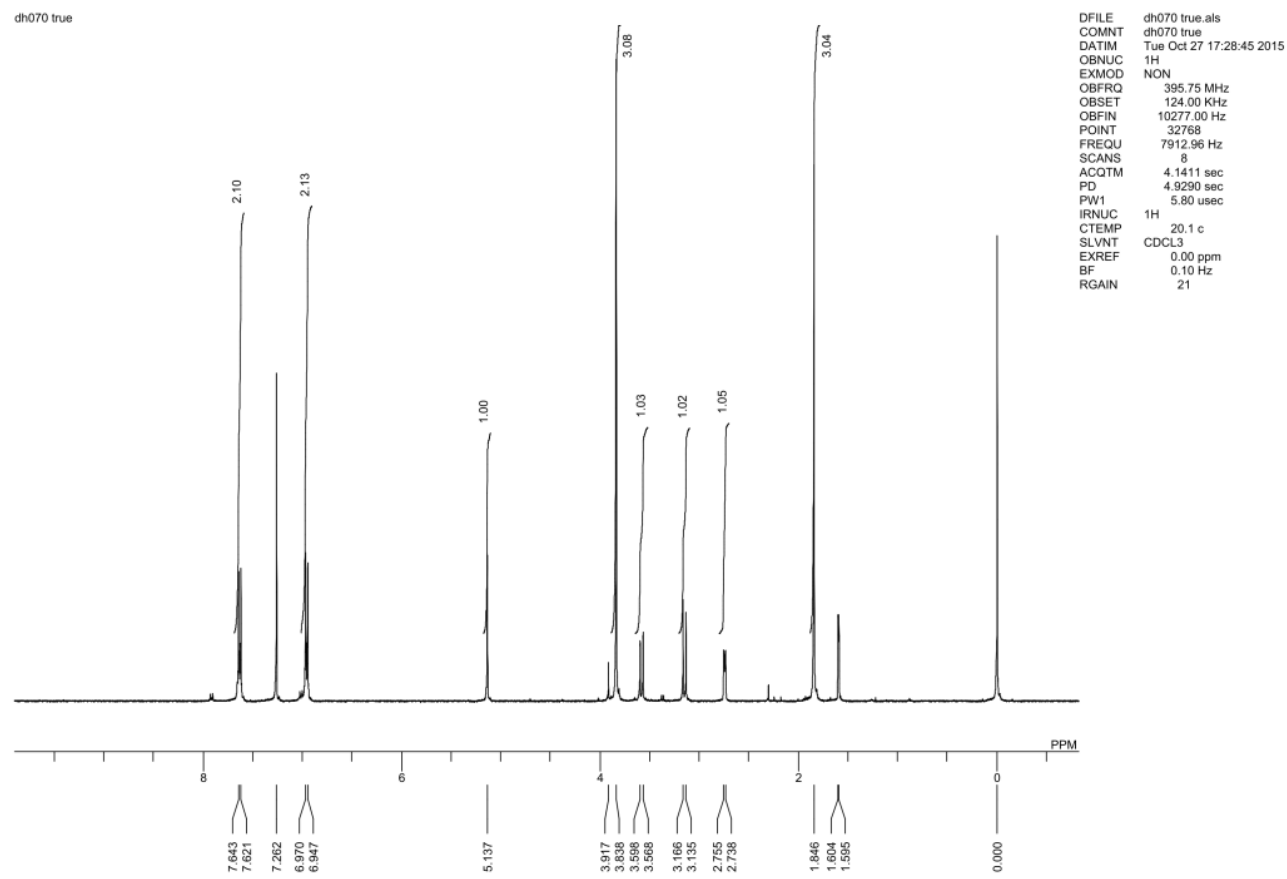


${ }^{13} \mathrm{C}$ NMR
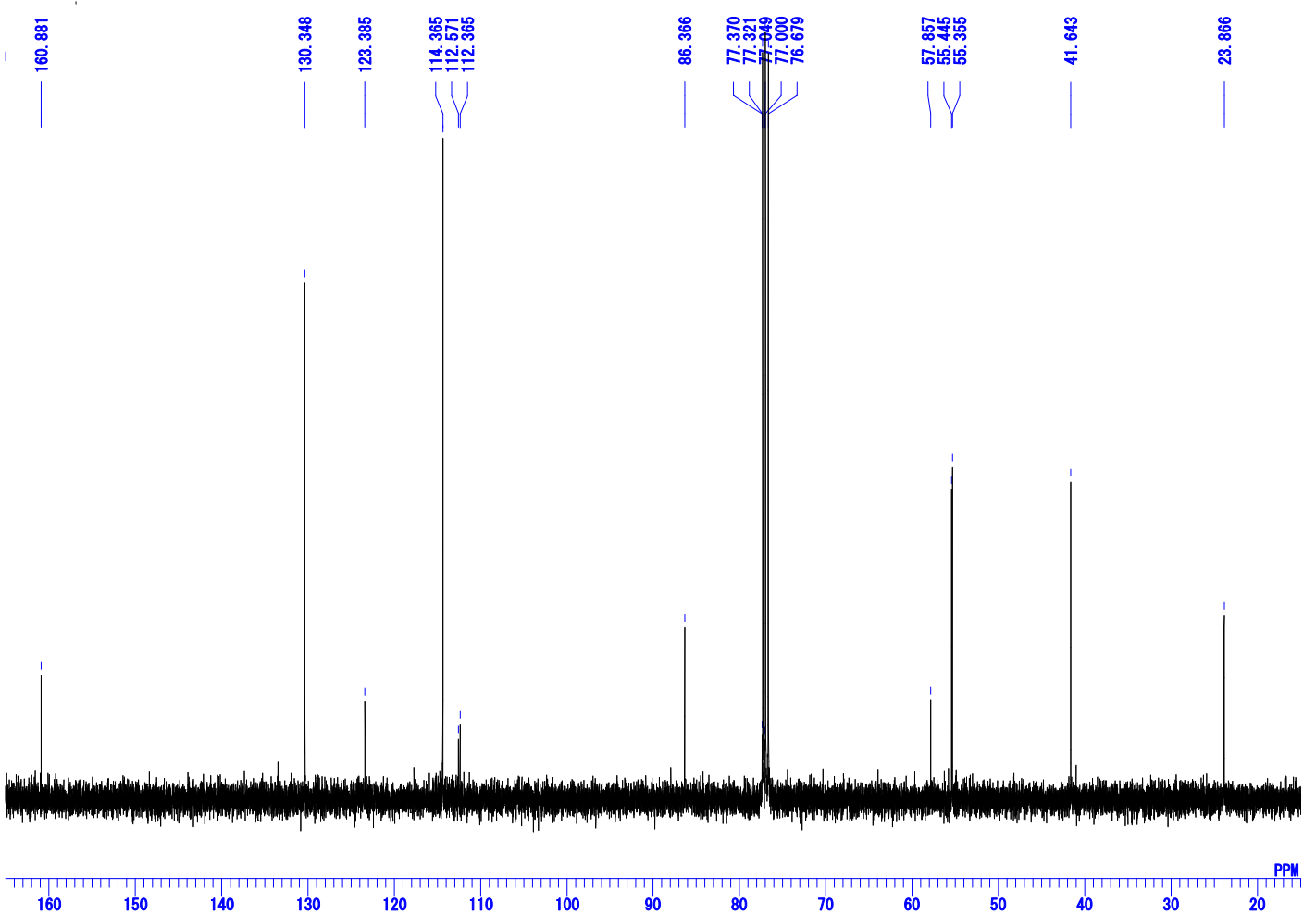
4-Hydroxy-4-methyl-2-(naphthalen-1-yl)dihydrothiophene-3,3(2H)-dicarbonitrile (4ae)<smiles>CC1(O)CSC(c2cccc3ccccc23)[C@@H]1C#N</smiles>

${ }^{1} \mathrm{H}$ NMR

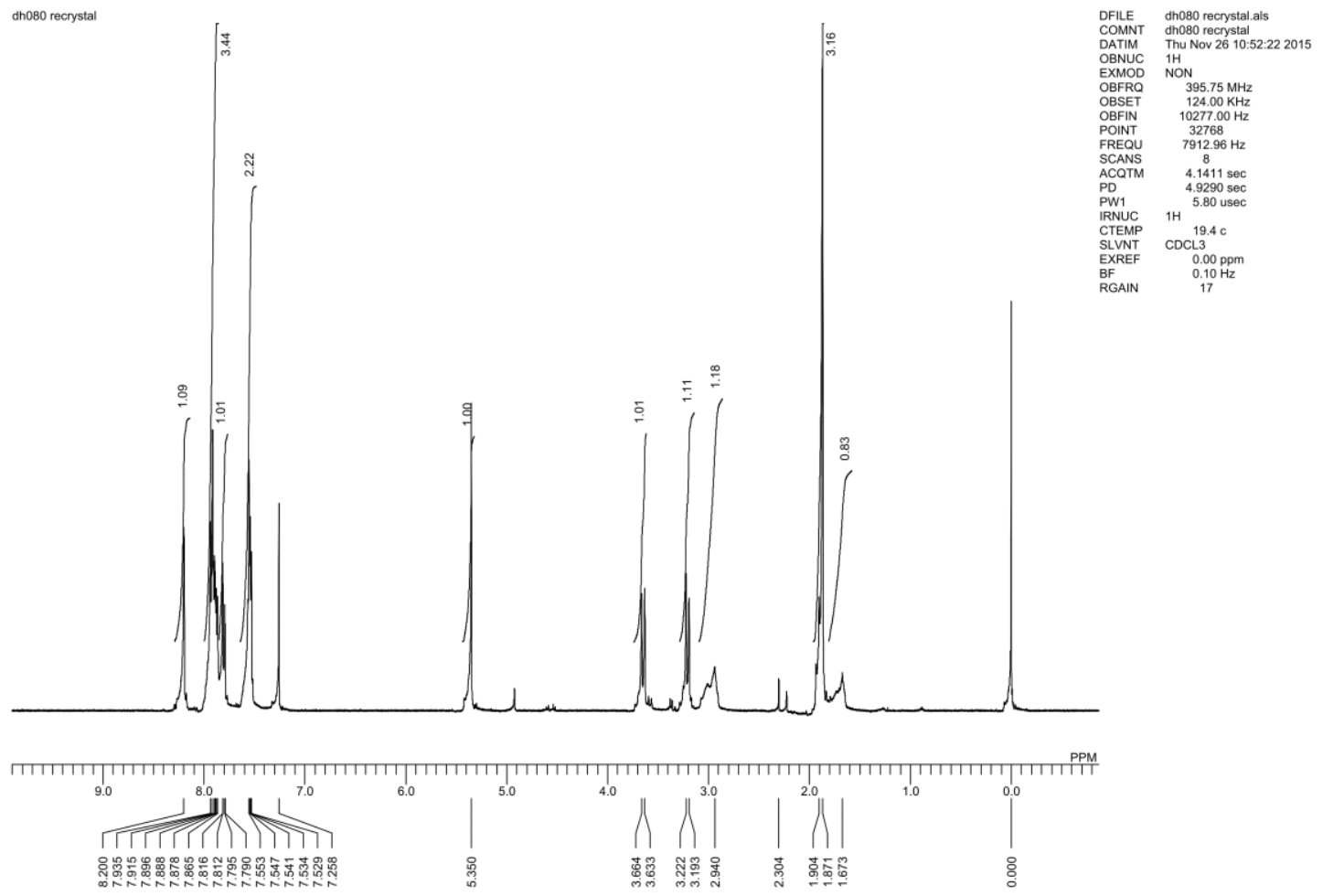


${ }^{13} \mathrm{C}$ NMR

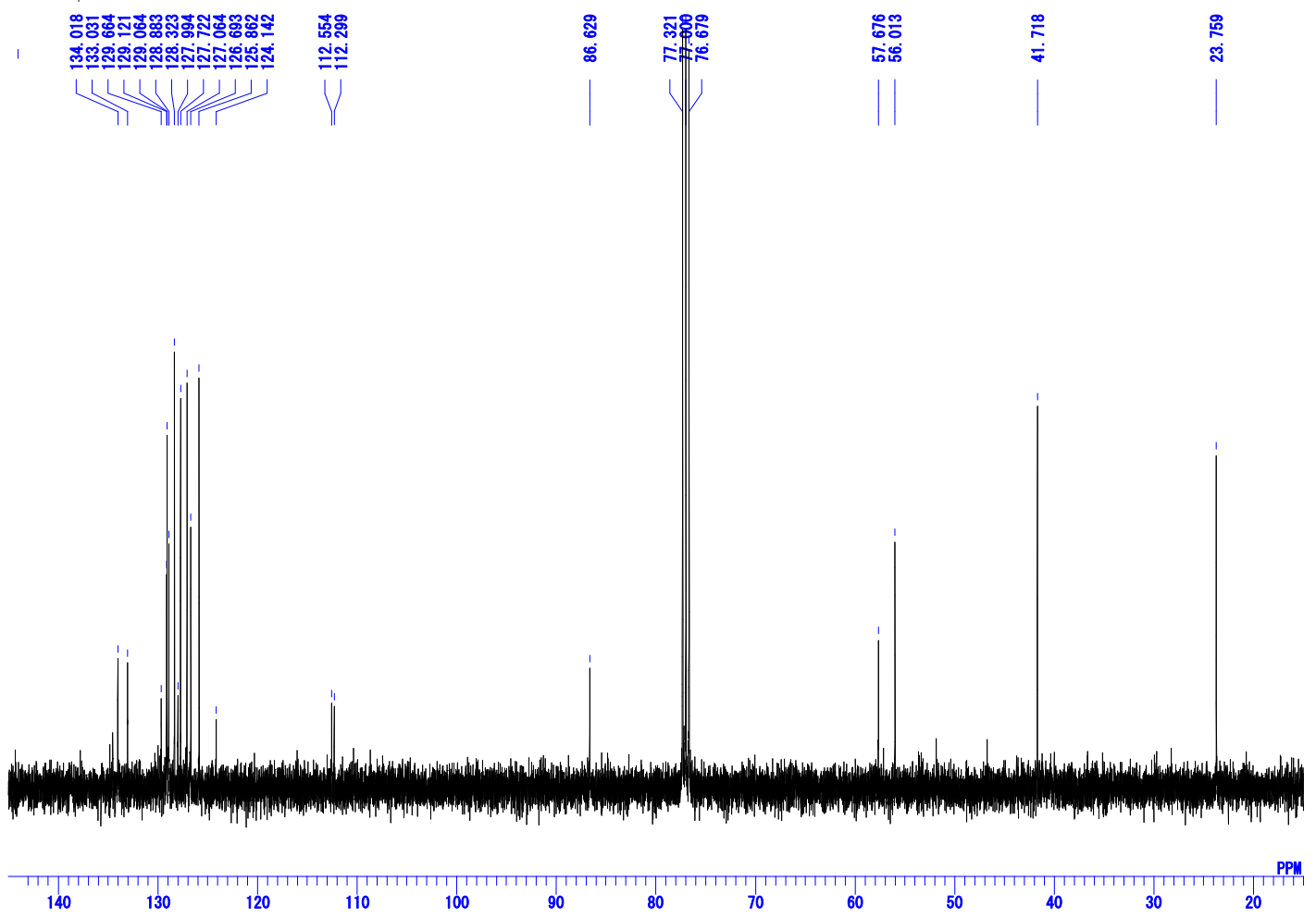


4-Hydroxy-4-methyl-2-phenyldihydrothiophene-3,3(2H)-dicarbonitrile (4af)<smiles>C[C@]1(O)CS[C@@H](c2ccco2)C1(C#N)C#N</smiles>

${ }^{1} \mathrm{H}$ NMR

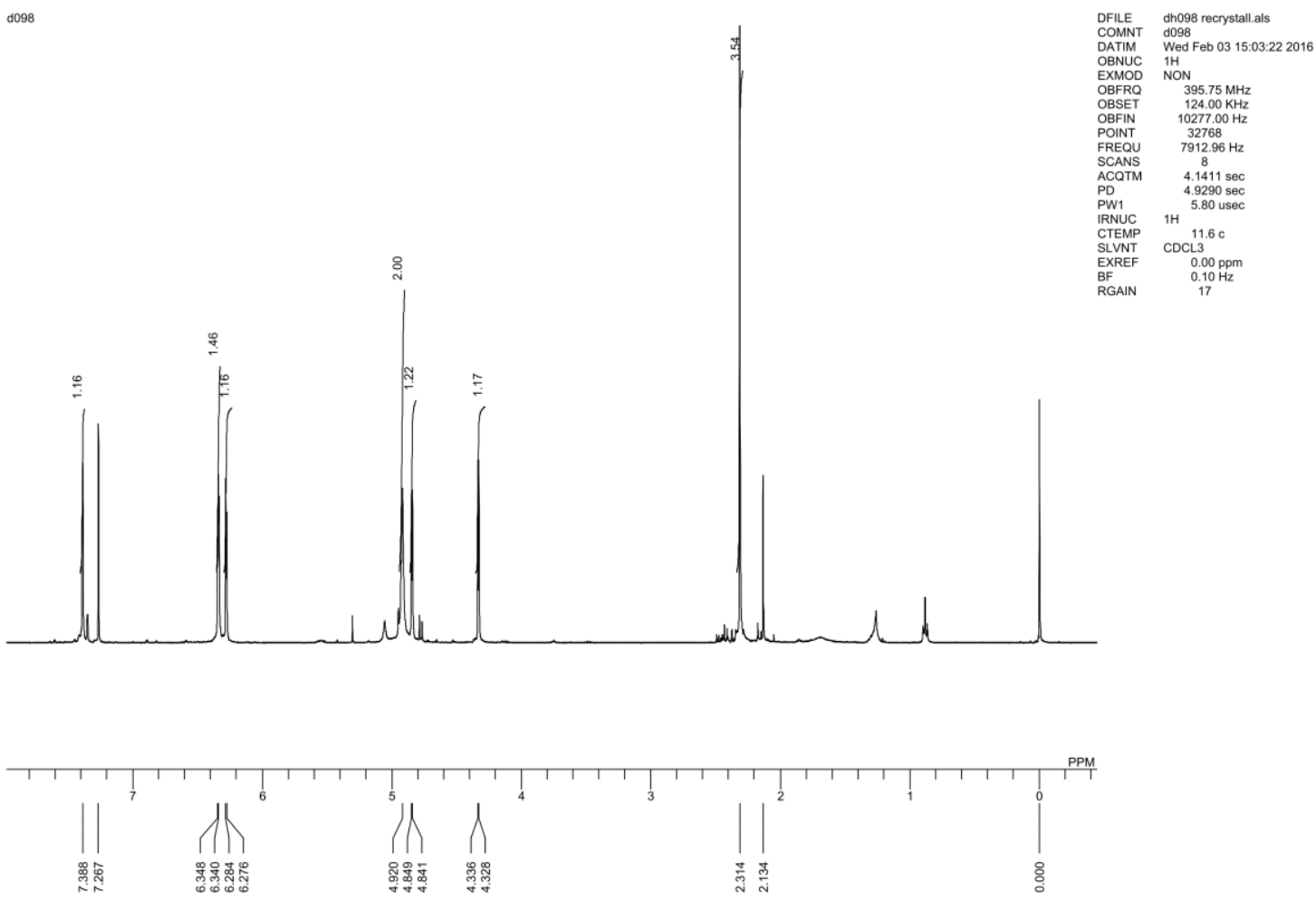


${ }^{13} \mathrm{C}$ NMR
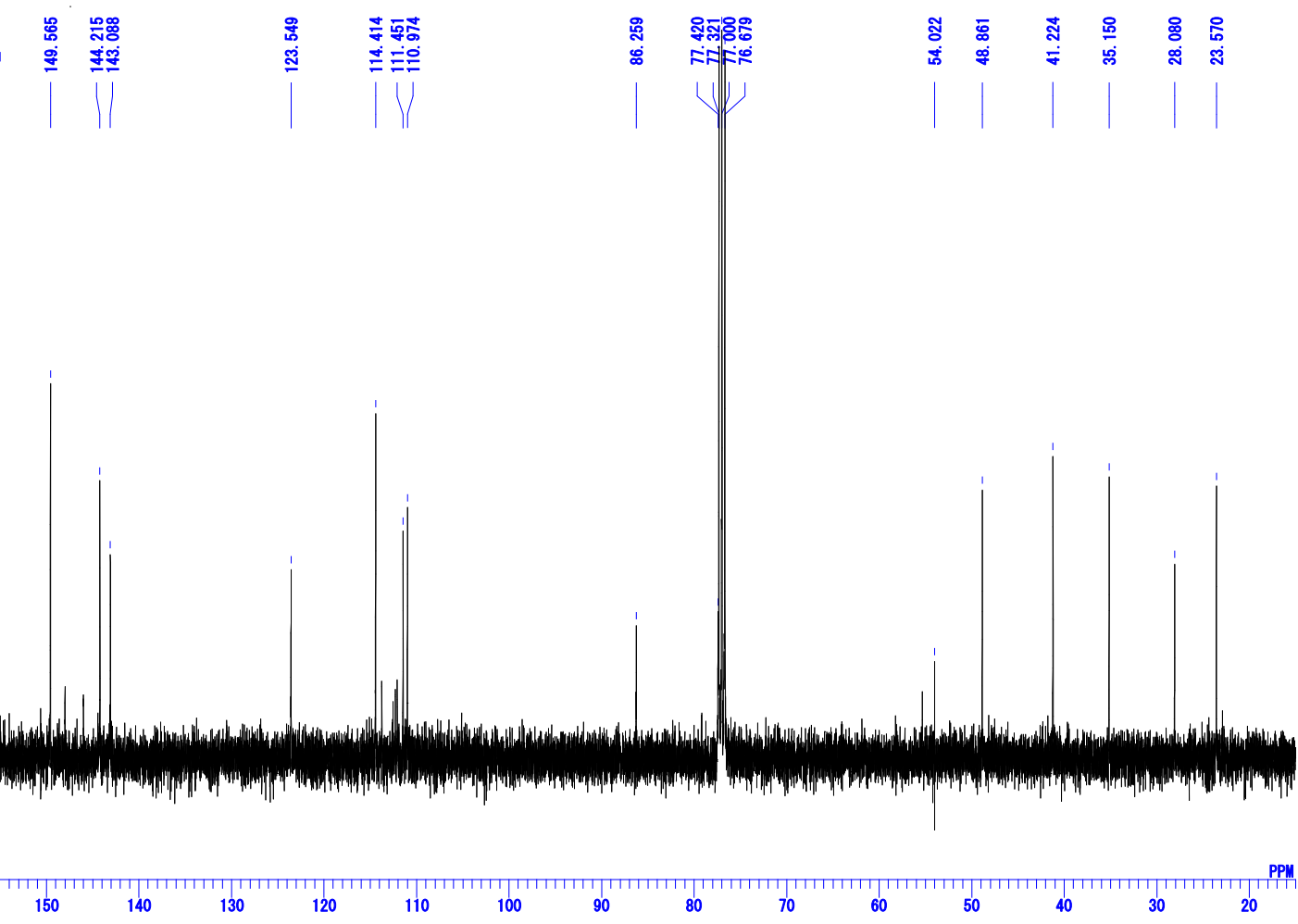


\section{4-Hydroxy-4,5-dimethyl-2-phenyldihydrothiophene-3,3(2H)-dicarbonitrile (4a'a)}

(CCDC; 1955807). This was recrystallized in hexane and EtOAc. ORTEP drawing is at the 50\% probability level.<smiles>CC1S[C@@H](c2ccccc2)C(C)(C#N)[C@@]1(C)O</smiles>

${ }^{1} \mathrm{H}$ NMR

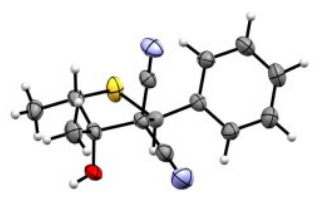

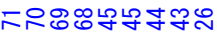
intivitivi $\Rightarrow \rightarrow$
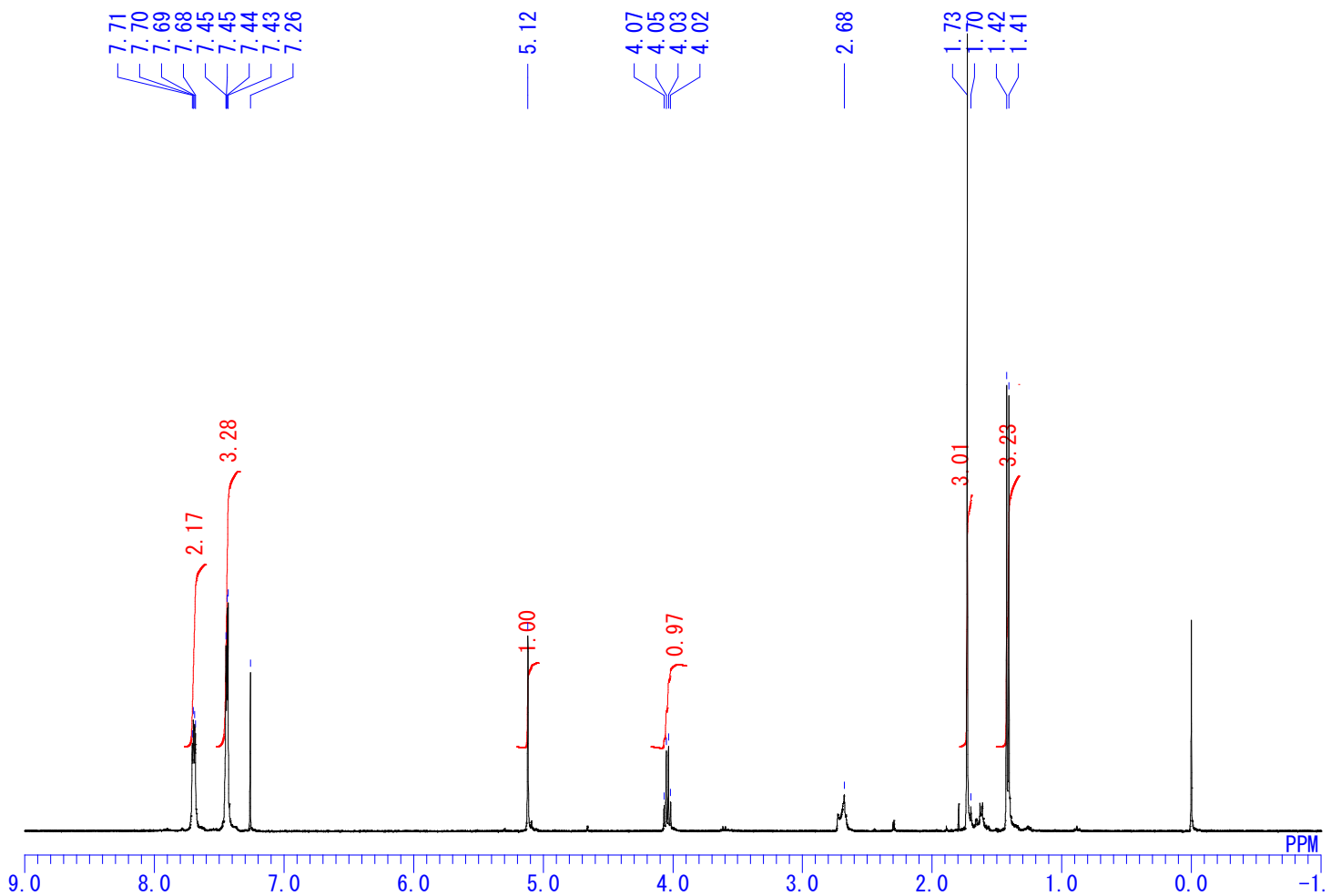
${ }^{13} \mathrm{C}$ NMR

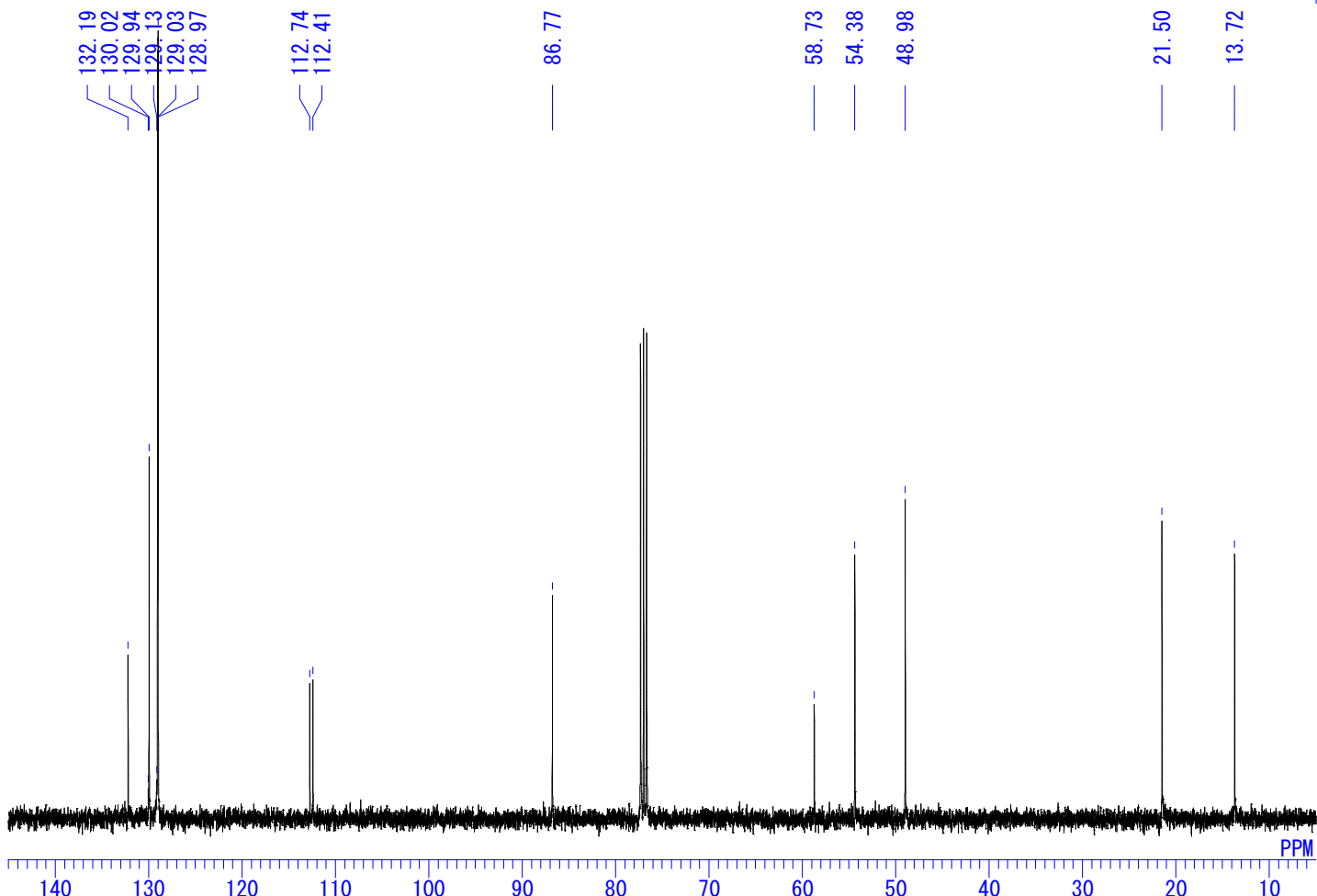


Crystal data and structure solution paramaters

\begin{tabular}{|c|c|c|c|}
\hline $\begin{array}{l}\text { Empirical } \\
\text { Formula }\end{array}$ & $\mathrm{C}_{14} \mathrm{H}_{14} \mathrm{~N}_{2} \mathrm{OS}$ & Function Minimized & $\Sigma \mathrm{w}\left(\mathrm{Fo}^{2}-\mathrm{Fc}^{2}\right)^{2}$ \\
\hline Formula Weight & 258.34 & $\begin{array}{l}\text { Least Squares } \\
\text { Weights }\end{array}$ & $1 /\left[0.0013 \mathrm{Fo}^{2}+1.0000 \sigma\left(\mathrm{Fo}^{2}\right)\right] /\left(4 \mathrm{Fo}^{2}\right)$ \\
\hline $\begin{array}{l}\text { Crystal Color, } \\
\text { Habit }\end{array}$ & colorless, block & $\begin{array}{l}\text { No. Observations (I } \\
>2.00 \sigma(\mathrm{I}))\end{array}$ & 2418 \\
\hline $\begin{array}{l}\text { Crystal } \\
\text { Dimensions }\end{array}$ & $\begin{array}{l}0.500 \times 0.200 \times 0.200 \\
\mathrm{~mm}\end{array}$ & No. Variables & 214 \\
\hline Crystal System & orthorhombic & $\begin{array}{l}\text { Reflection/Parameter } \\
\text { Ratio }\end{array}$ & 11.30 \\
\hline \multirow[t]{6}{*}{ Lattice Type } & Primitive & $\begin{array}{l}\text { Residuals: R } \\
(\mathrm{I}>2.00 \sigma(\mathrm{I}))\end{array}$ & 0.0330 \\
\hline & $a=8.1671(2) \AA$ & $\begin{array}{l}\text { Residuals: wR } \\
(\mathrm{I}>2.00 \sigma(\mathrm{I}))\end{array}$ & 0.0893 \\
\hline & $\mathrm{b}=11.2610(2) \AA$ & $\begin{array}{l}\text { Goodness of Fit } \\
\text { Indicator }\end{array}$ & 1.021 \\
\hline & $\mathrm{c}=14.2325(3) \AA$ & $\begin{array}{l}\text { Max Shift/Error in } \\
\text { Final Cycle }\end{array}$ & 0.002 \\
\hline & $V=1308.97(5) \AA^{3}$ & $\begin{array}{l}\text { Maximum peak in } \\
\text { Final Diff. Map }\end{array}$ & $0.82 \mathrm{e}^{-/} \AA^{3}$ \\
\hline & & $\begin{array}{l}\text { Minimum peak in } \\
\text { Final Diff. Map }\end{array}$ & $-0.60 e^{-/} \AA^{3}$ \\
\hline Space Group & $\mathrm{P} 2{ }_{1}{ }_{1}{ }^{2}{ }_{1}(\# 19)$ & & \\
\hline$Z$ value & 4 & & \\
\hline$D_{\text {cal }}$ & $1.311 \mathrm{~g} / \mathrm{cm}^{3}$ & & \\
\hline$F_{000}$ & 544.00 & & \\
\hline$\mu(\mathrm{CuK} \alpha)$ & $21.044 \mathrm{~cm}^{-1}$ & & \\
\hline Temperature & $23.0^{\circ} \mathrm{C}$ & & \\
\hline
\end{tabular}




\section{2-(((2-Oxo-2-phenylethyl)thio)(phenyl)methyl)malononitrile (5ba)}

(This compound was decomposed during purification by chromatography, so rough data of ${ }^{1} \mathrm{H}$ NMR were shown)<smiles>N#CC(C#N)C(SCC(=O)c1ccccc1)c1ccccc1</smiles>

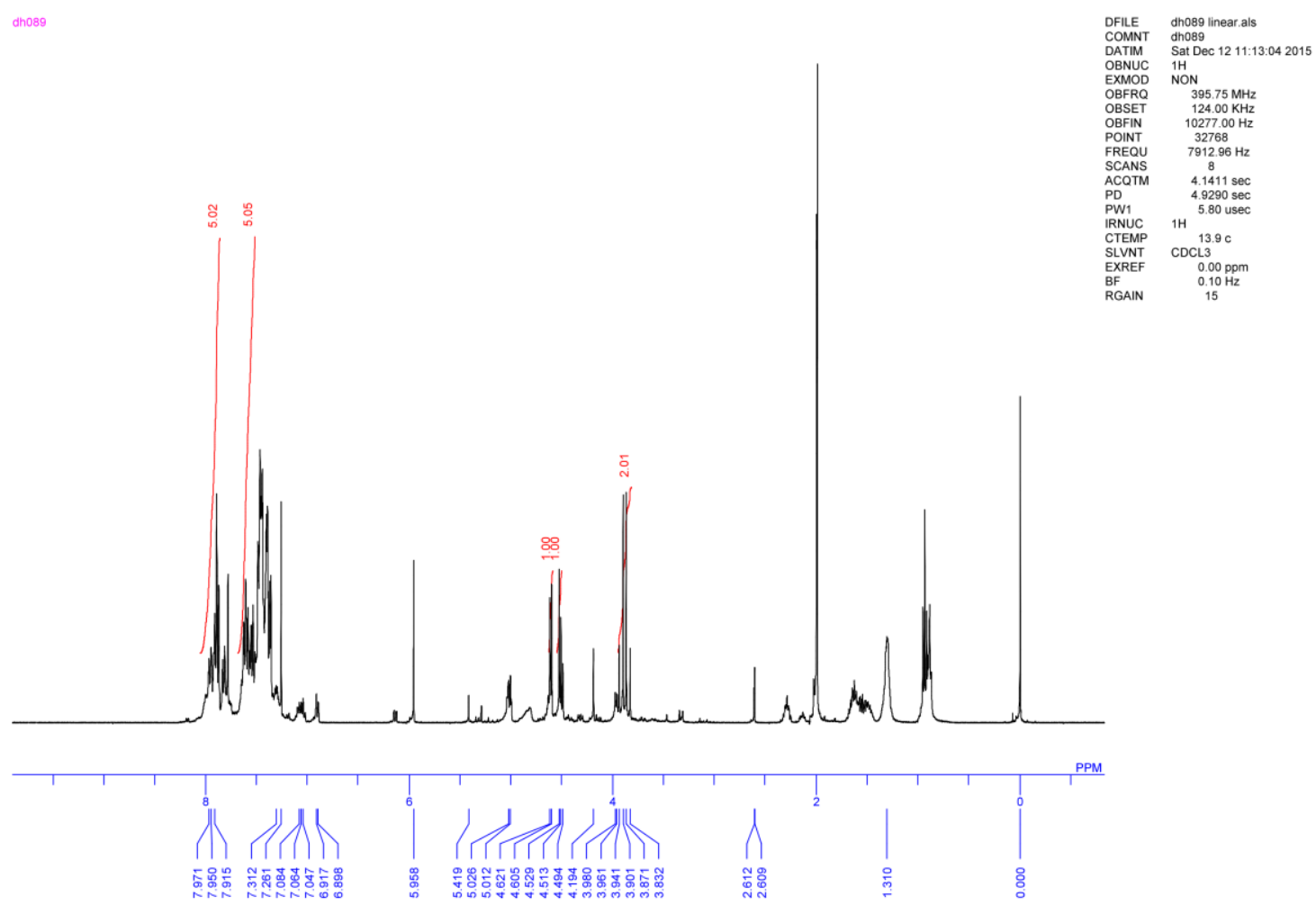


Dethyl 2-(((2-oxopropyl)thio)(phenyl)methyl)malonate (5aj)<smiles>CCOC(=O)C(C(=O)OCC)C(SCC(C)=O)c1ccccc1</smiles>

${ }^{1} \mathrm{H}-\mathrm{NMR}$

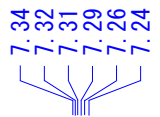

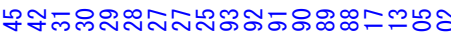

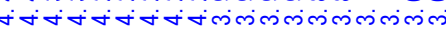
LLLLLL L U

๓ 4n -i-..000000 LU H

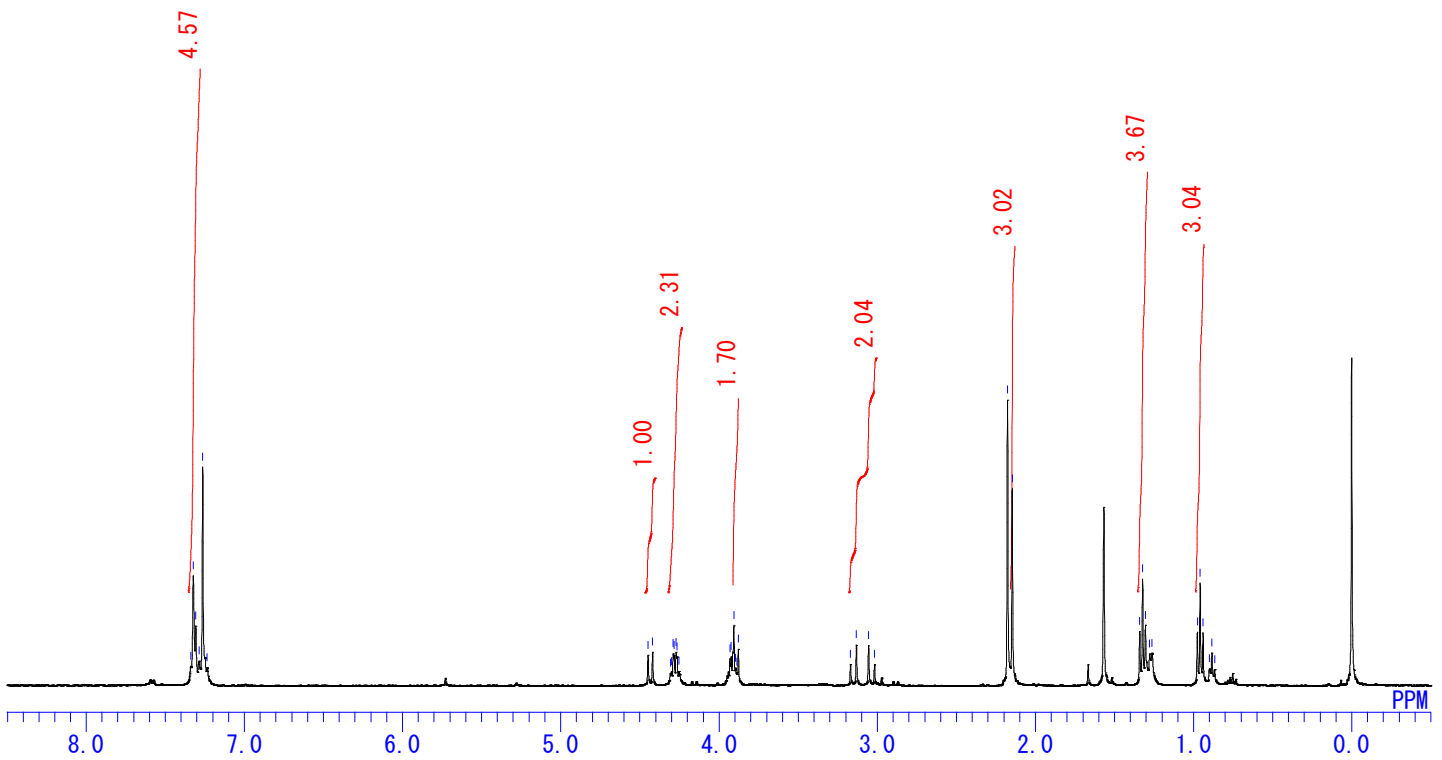


${ }^{13} \mathrm{C}$ NMR

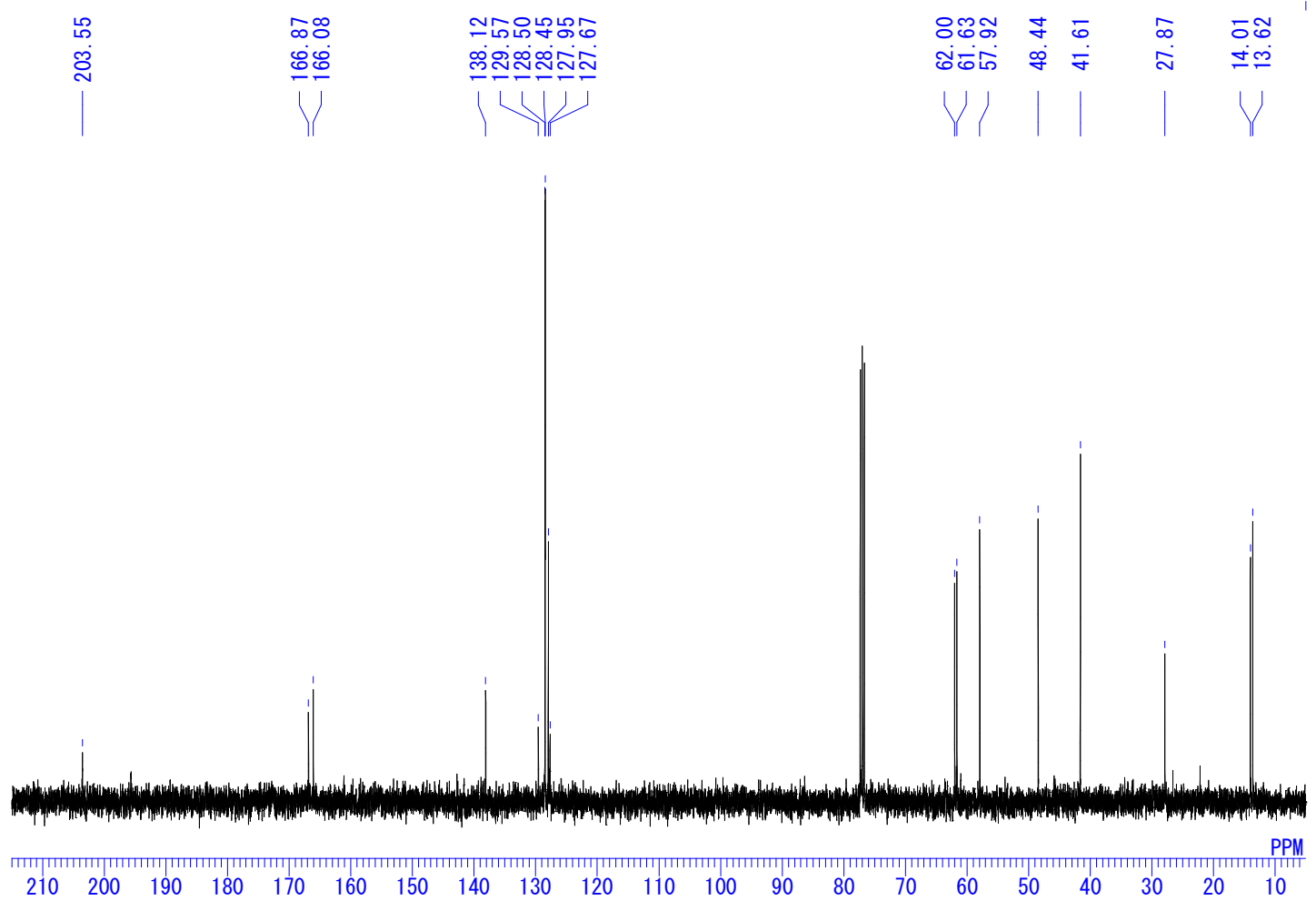


Diethyl 2-(((2-oxo-2-phenylethyl)thio)(phenyl)methyl)malonate (5cj)<smiles>CCOC(=O)C(C(=O)OCC)C(SCC(=O)c1ccccc1)c1ccccc1</smiles>

${ }^{1} \mathrm{H}-\mathrm{NMR}$

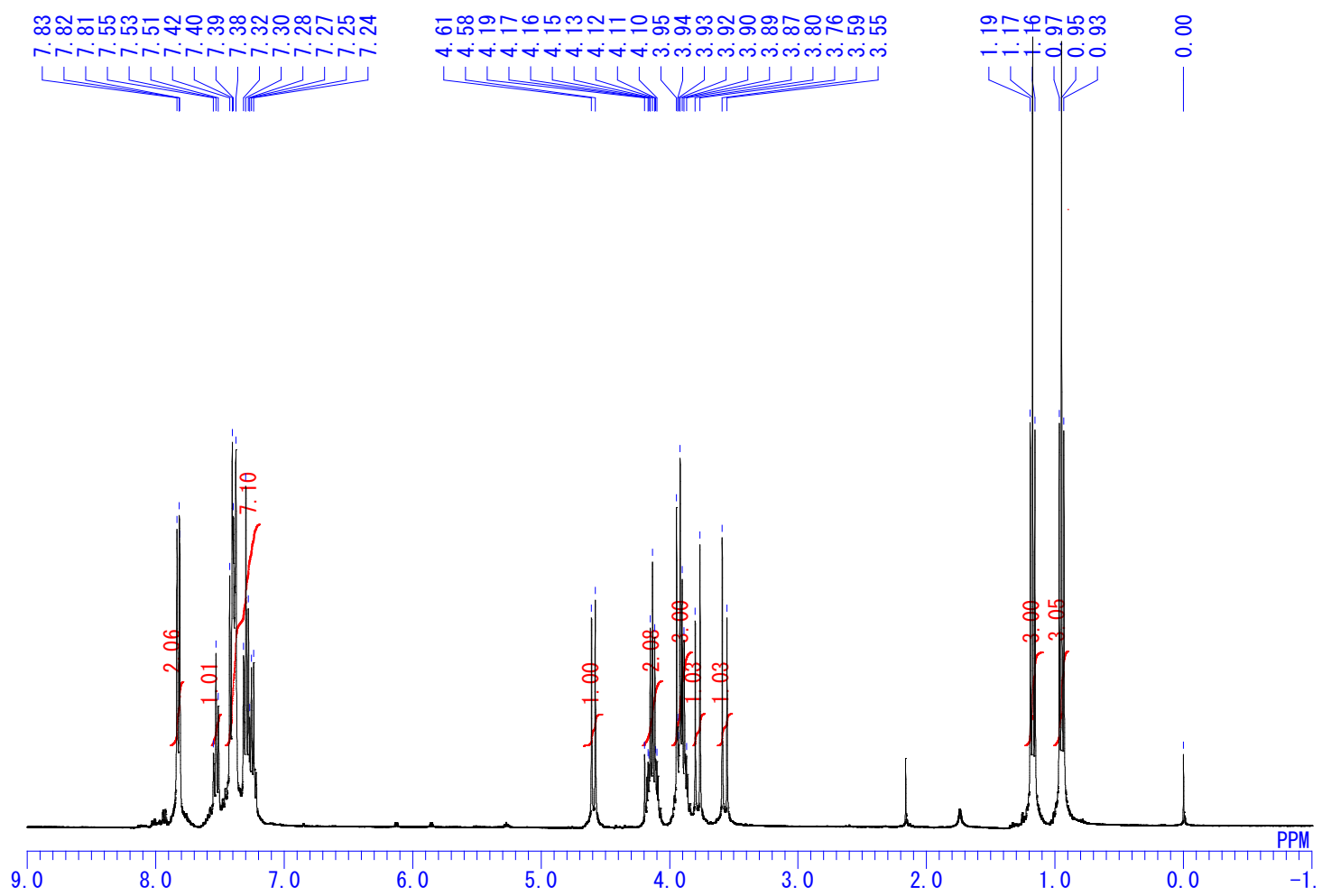


${ }^{13} \mathrm{C}-\mathrm{NMR}$

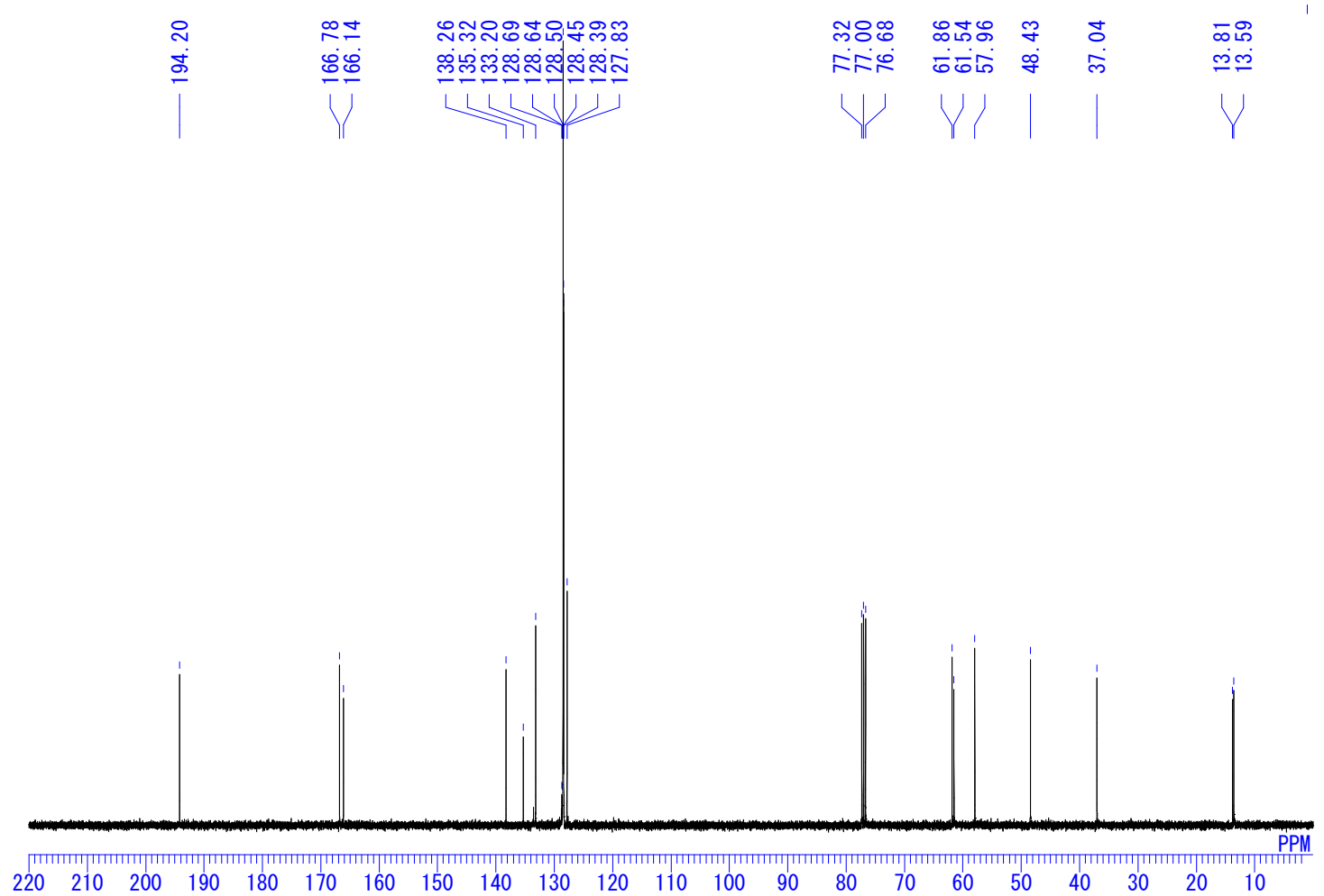


Methyl 5-benzoyl-2-oxo-4-phenyltetrahydrothiophene-3-carboxylate (6ci)

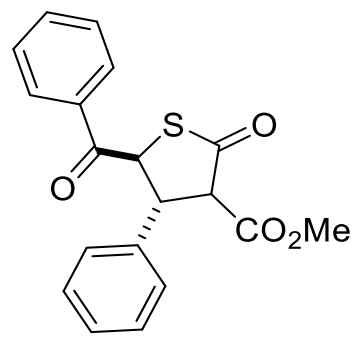

${ }^{1} \mathrm{H}-\mathrm{NMR}$

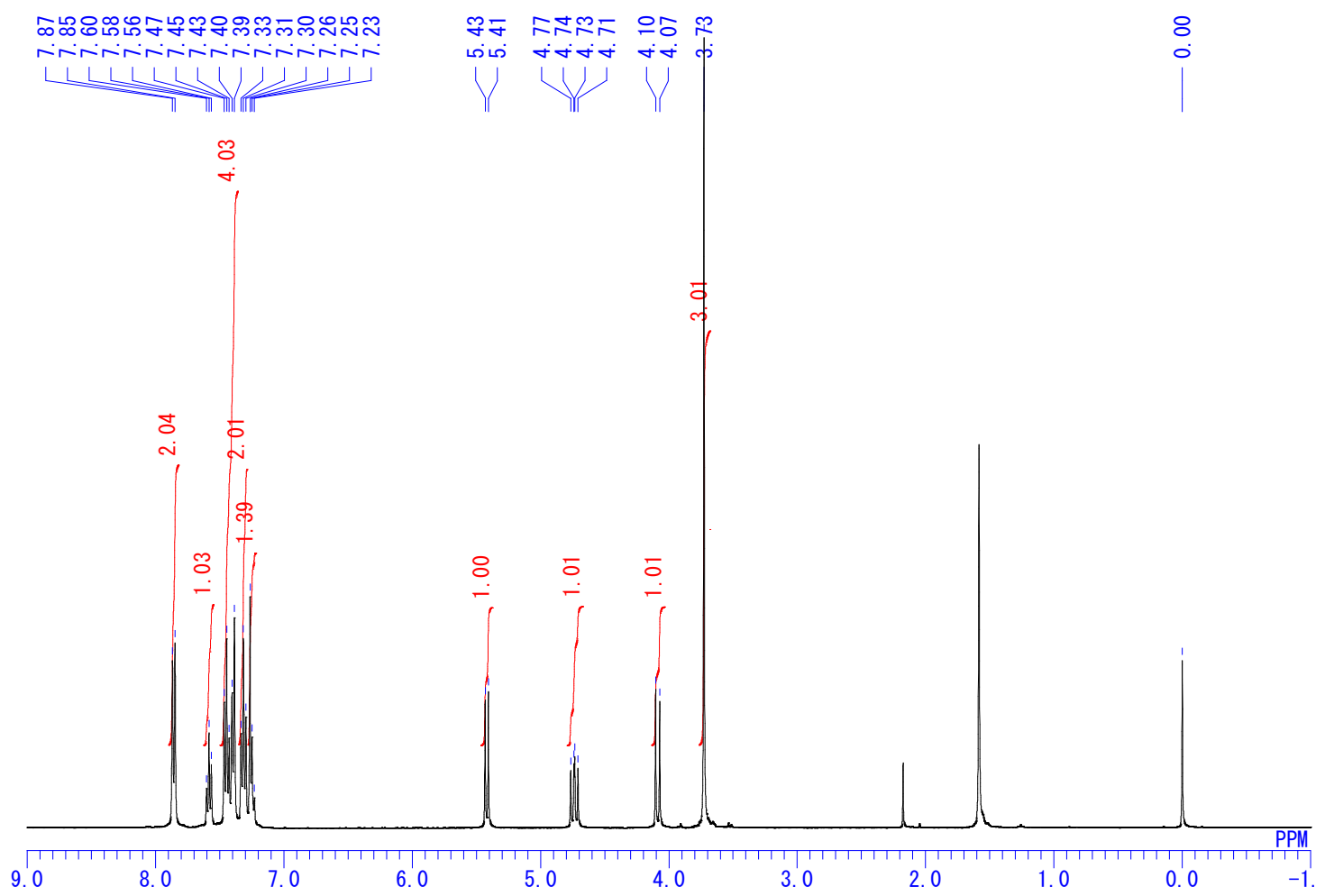


${ }^{13} \mathrm{C}-\mathrm{NMR}$

ณี

호 ㅇำ

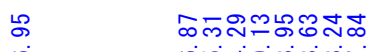

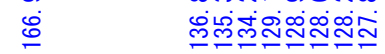

나워

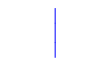

|

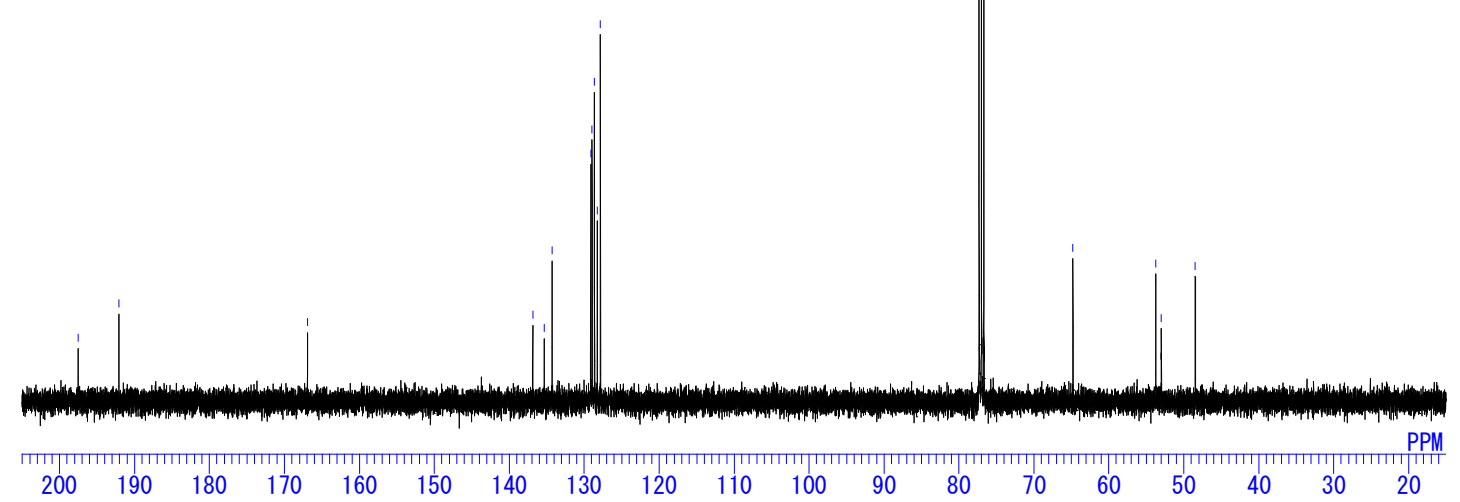


Dr mixture

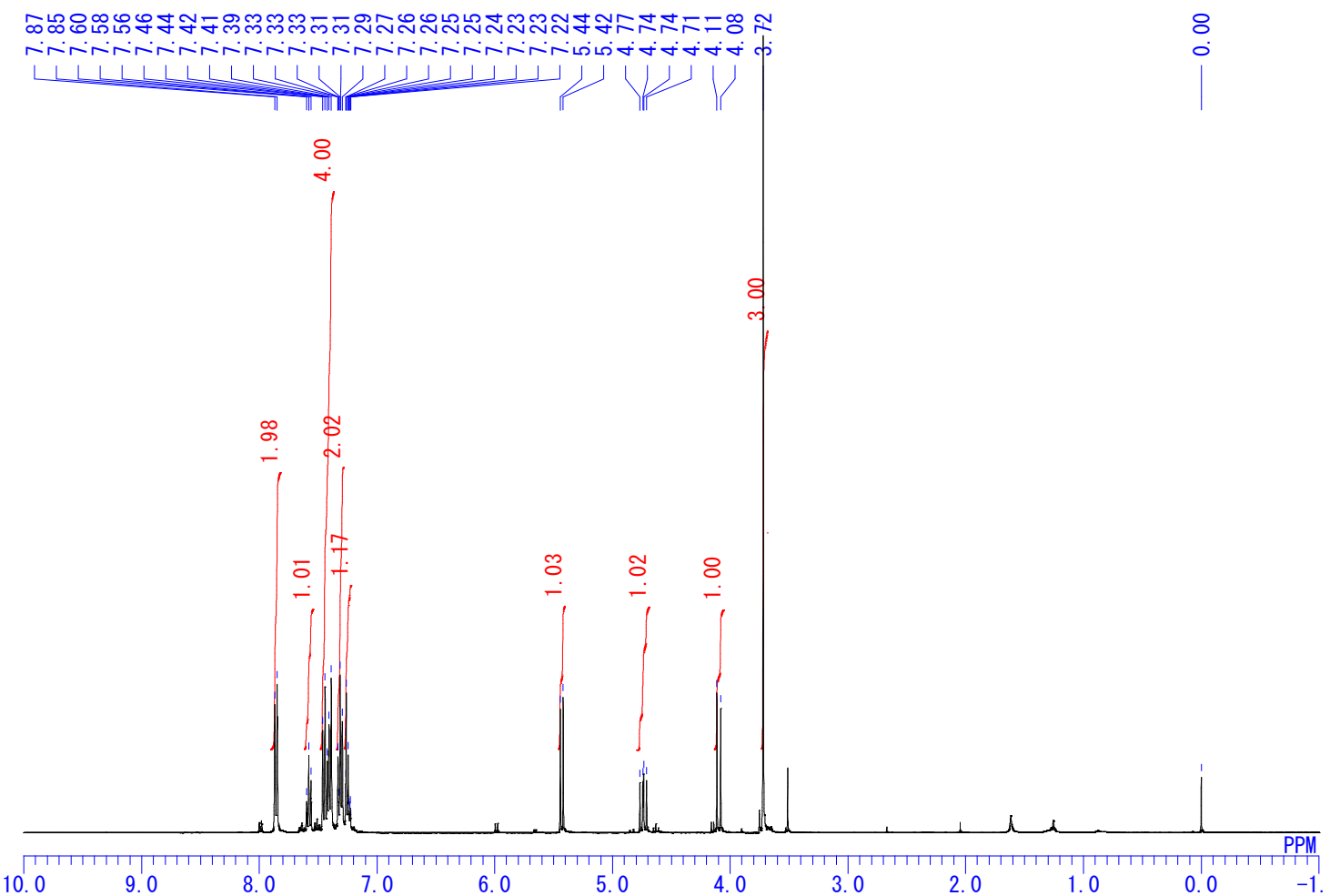


Methyl 5-benzoyl-3-hydroxy-2-oxo-4-phenyltetrahydrothiophene-3-carboxylate (8ci)

(CCDC; 1964458). This was recrystallized in hexane and EtOAc. ORTEP drawing is at the 50\% probability level.
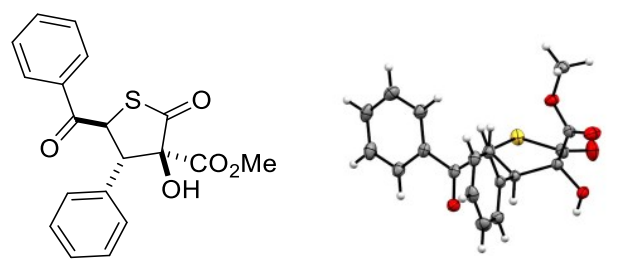

${ }^{1} \mathrm{H}-\mathrm{NMR}$

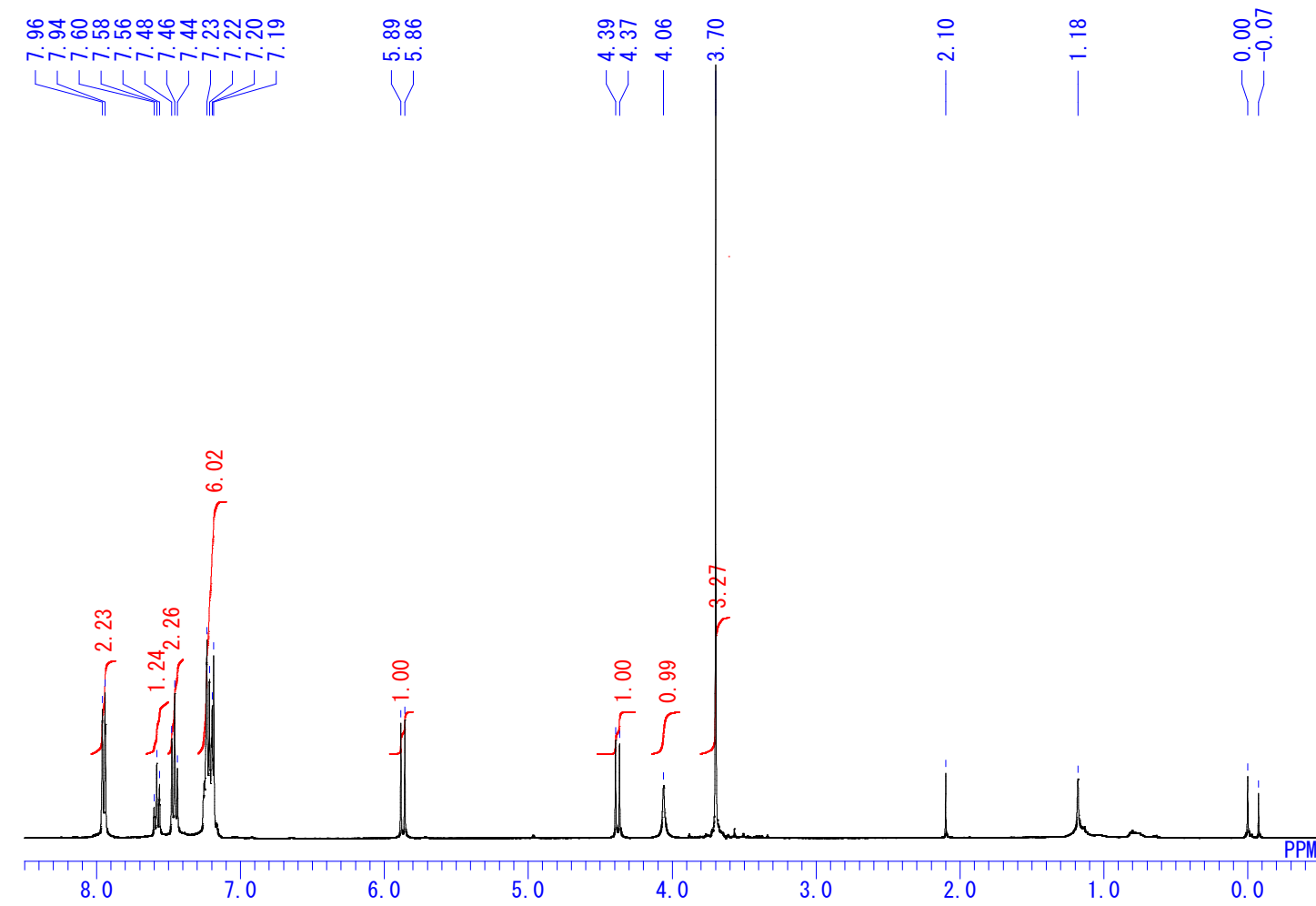


${ }^{13} \mathrm{C}-\mathrm{NMR}$

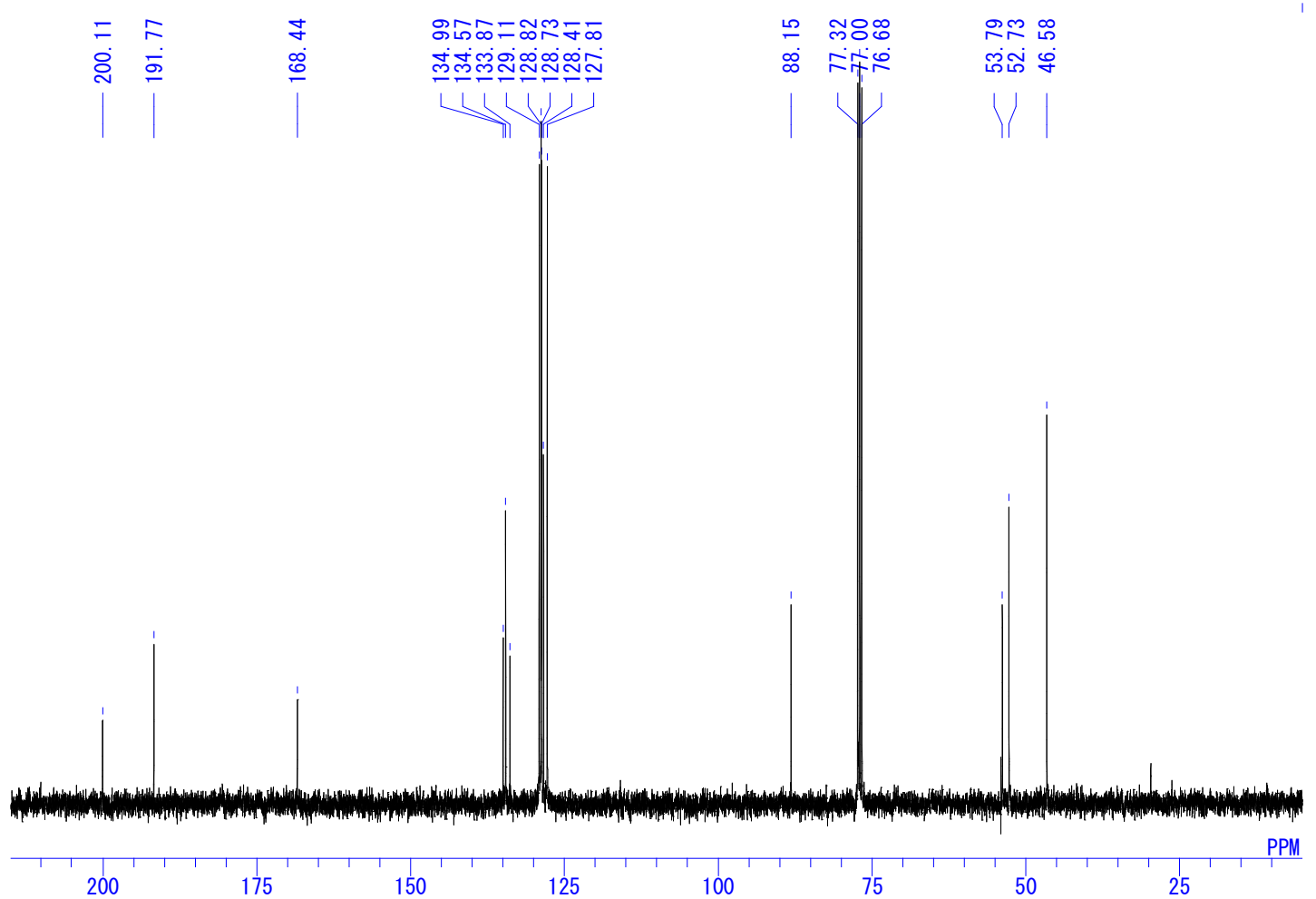


Crystal data and structure solution paramaters

\begin{tabular}{|c|c|c|c|}
\hline $\begin{array}{l}\text { Empirical } \\
\text { Formula }\end{array}$ & $\mathrm{C}_{19} \mathrm{H}_{16} \mathrm{O}_{5} \mathrm{~S}$ & Function Minimized & $\Sigma \mathrm{w}\left(\mathrm{Fo}^{2}-\mathrm{Fc}^{2}\right)^{2}$ \\
\hline $\begin{array}{l}\text { Formula } \\
\text { Weight }\end{array}$ & 356.39 & $\begin{array}{l}\text { Least Squares } \\
\text { Weights }\end{array}$ & $\begin{array}{l}\mathrm{w}=1 /\left[\sigma^{2}\left(\mathrm{Fo}^{2}\right)+(0.0445 \cdot \mathrm{P})^{2}+0.7857 \cdot \mathrm{P}\right] \\
\text { where } \mathrm{P}=\left(\operatorname{Max}\left(\mathrm{Fo}^{2}, 0\right)+2 \mathrm{Fc}^{2}\right) / 3\end{array}$ \\
\hline $\begin{array}{l}\text { Crystal Color, } \\
\text { Habit }\end{array}$ & translucentintense, block & $\begin{array}{l}\text { No. Observations } \\
\text { (All reflections) }\end{array}$ & 4192 \\
\hline $\begin{array}{l}\text { Crystal } \\
\text { Dimensions }\end{array}$ & $\begin{array}{l}0.114 \times 0.088 \times 0.061 \\
\mathrm{~mm}\end{array}$ & No. Variables & 226 \\
\hline Crystal System & monoclinic & $\begin{array}{l}\text { Reflection/Paramete } \\
\text { r Ratio }\end{array}$ & 18.55 \\
\hline \multirow[t]{7}{*}{ Lattice Type } & Primitive & $\begin{array}{l}\text { Residuals: R1 } \\
(\mathrm{I}>2.00 \sigma(\mathrm{I}))\end{array}$ & 0.0415 \\
\hline & $\mathrm{a}=8.9029(4) \AA$ & $\begin{array}{l}\text { Residuals: R (All } \\
\text { reflections) }\end{array}$ & 0.0620 \\
\hline & $\mathrm{b}=18.4381(8) \AA$ & $\begin{array}{l}\text { Residuals: wR2 (All } \\
\text { reflections) }\end{array}$ & 0.1065 \\
\hline & $\mathrm{c}=10.5002(5) \AA$ & $\begin{array}{l}\text { Goodness of Fit } \\
\text { Indicator }\end{array}$ & 1.029 \\
\hline & $V=1677.93(19) \AA^{3}$ & $\begin{array}{l}\text { Max Shift/Error in } \\
\text { Final Cycle }\end{array}$ & 0.000 \\
\hline & & $\begin{array}{l}\text { Maximum peak in } \\
\text { Final Diff. Map }\end{array}$ & $0.37 \mathrm{e}^{-} / \AA^{3}$ \\
\hline & & $\begin{array}{l}\text { Minimum peak in } \\
\text { Final Diff. Map }\end{array}$ & $-0.25 \mathrm{e}^{-} / \AA^{3}$ \\
\hline Space Group & $\mathrm{P} 2{ }_{1} / \mathrm{c}(\# 14)$ & & \\
\hline$Z$ value & 4 & & \\
\hline$D_{\text {cal }}$ & $1.423 \mathrm{~g} / \mathrm{cm}^{3}$ & & \\
\hline$F_{000}$ & 744.00 & & \\
\hline$\mu(\operatorname{MoK} \alpha)$ & $2.217 \mathrm{~cm}^{-1}$ & & \\
\hline Temperature & $-150.0^{\circ} \mathrm{C}$ & & \\
\hline
\end{tabular}


2-Amino-5-benzoyl-4-phenylthiophene-3-carbonitrile (9ca)

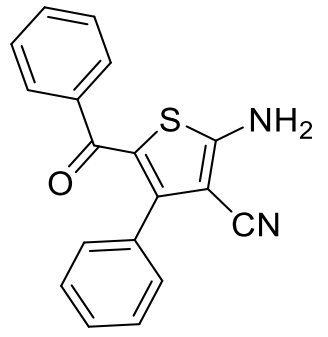

${ }^{1} \mathrm{H}-\mathrm{NMR}$

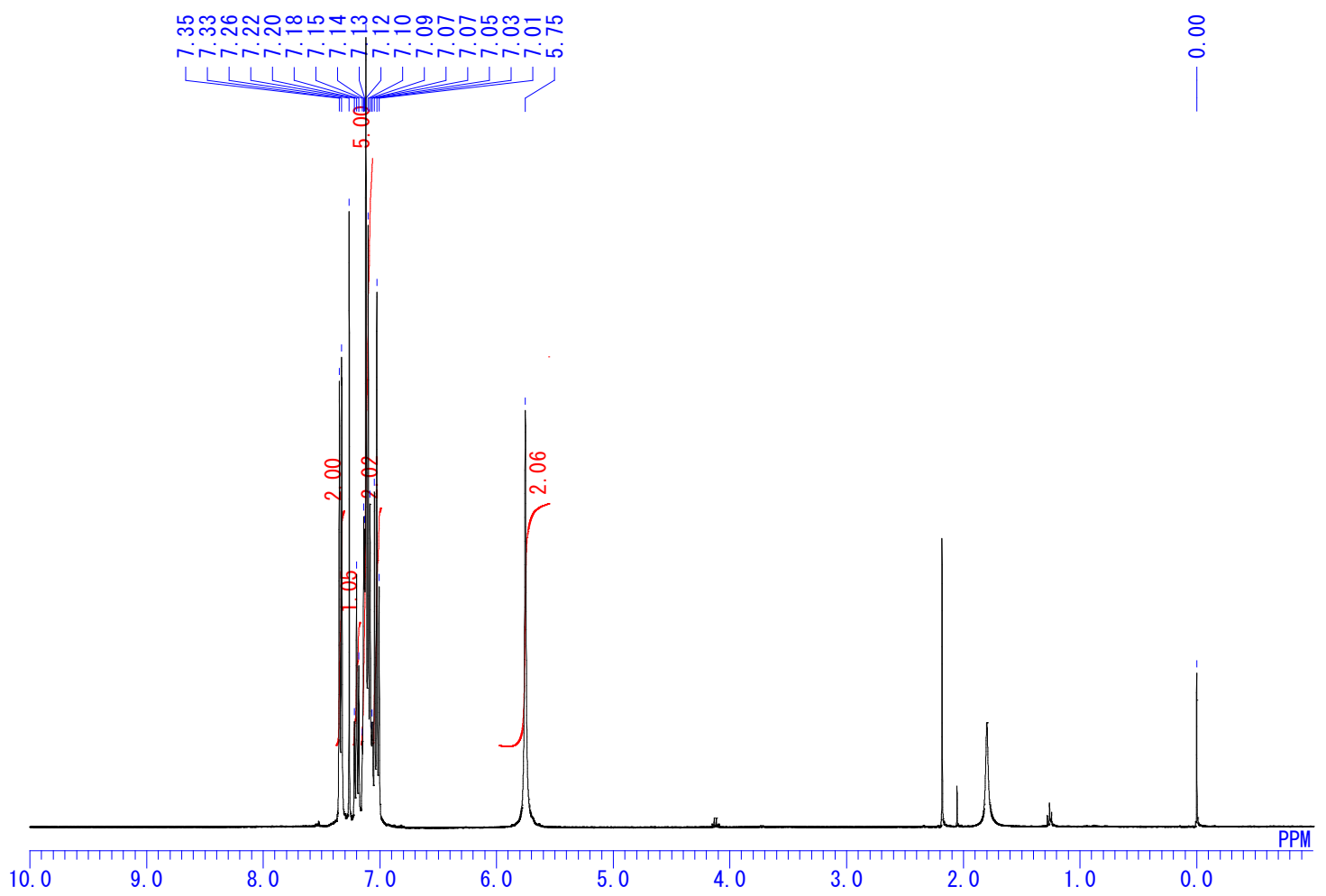


${ }^{13} \mathrm{C}-\mathrm{NMR}$

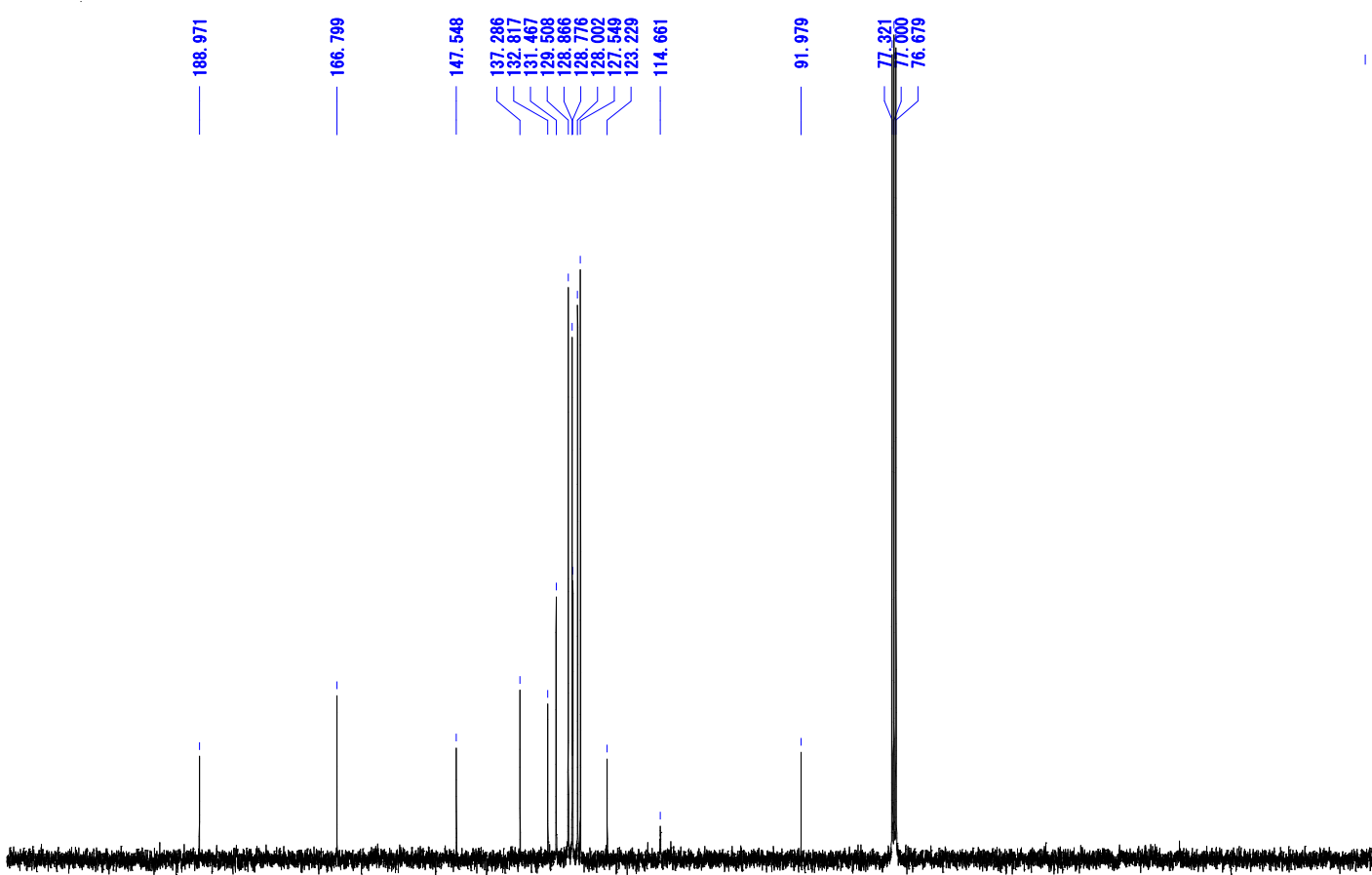

$\begin{array}{llllllllllllll}220 & 210 & 200 & 190 & 180 & 170 & 160 & 150 & 140 & 130 & 120 & 110 & 100\end{array}$ 
Methyl 2-amino-5-benzoyl-4-phenylthiophene-3-carboxylate (9ci)

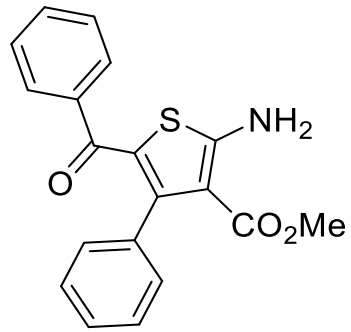

${ }^{1} \mathrm{H}-\mathrm{NMR}$

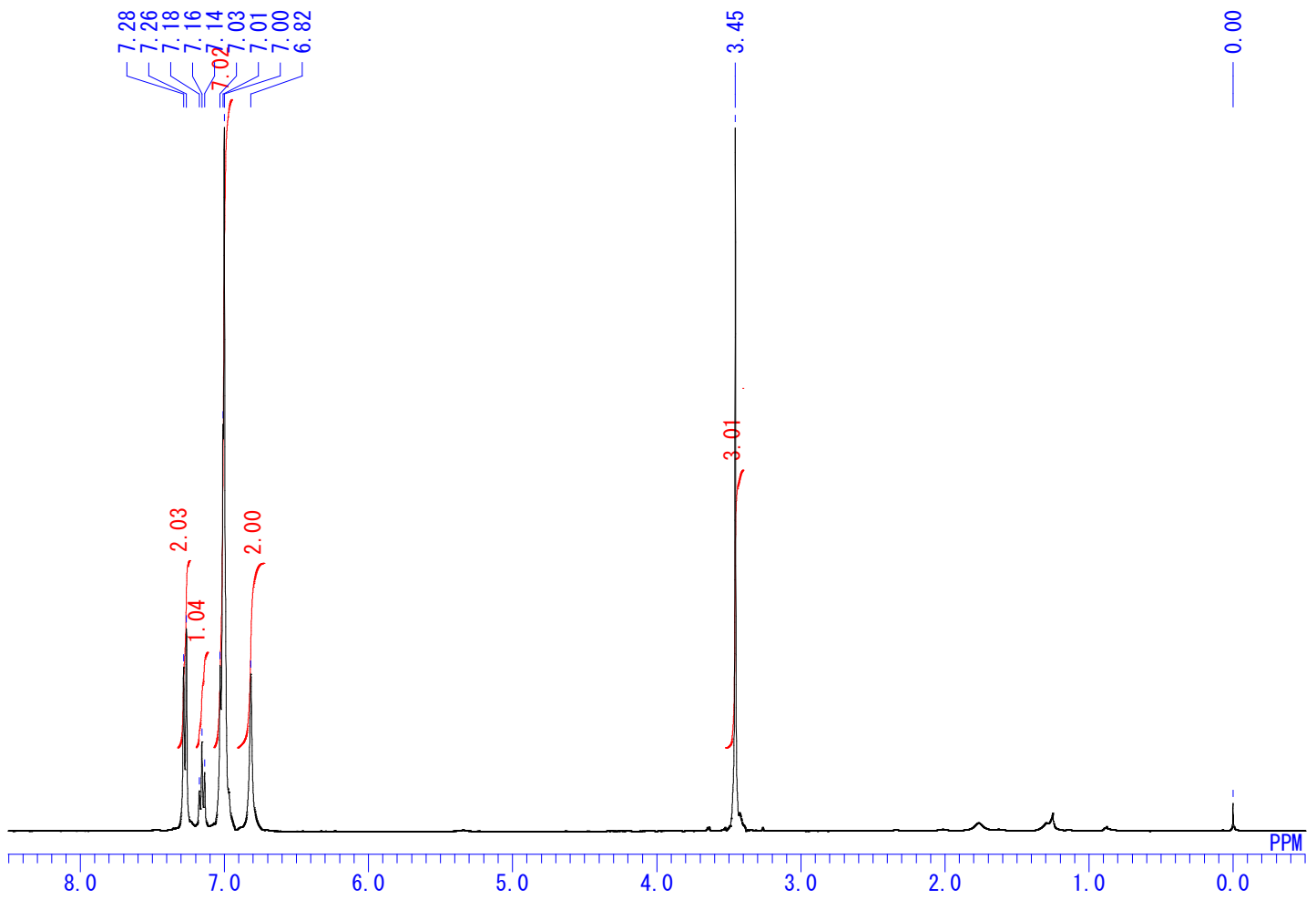


${ }^{13} \mathrm{C}-\mathrm{NMR}$

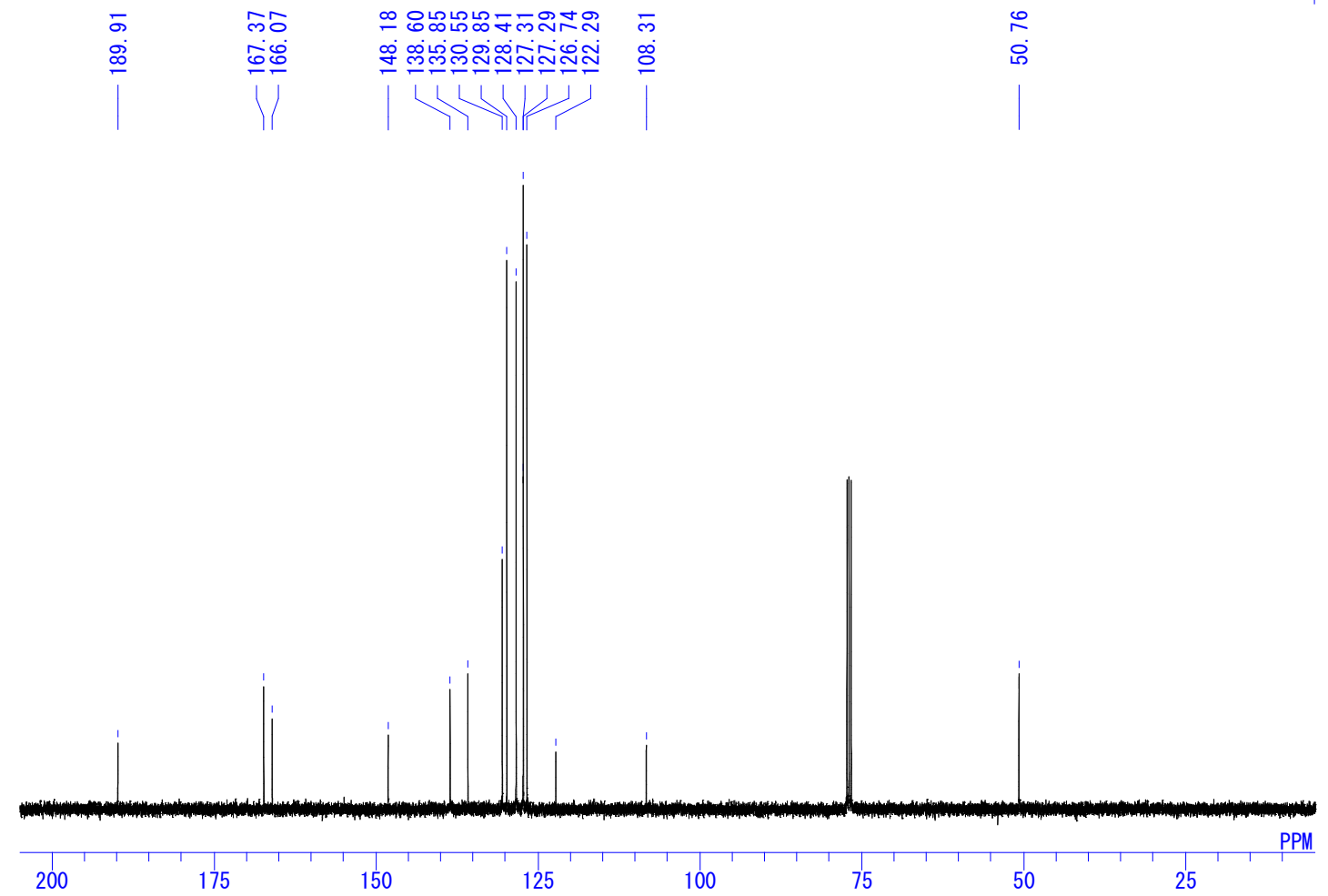




\section{Discussion}

\section{Diastereoselectivity of the dihydrothiophene 3}

The trans-selectivities of the dihydrothiophenes 3 could be dependent upon the substituent $\mathrm{R}^{1}$ of mercapto ketones $\mathbf{1}$. The anti-selectivity was determined during the formation of $\mathrm{C}-\mathrm{C}$ bonds between in situ-generated tin enolate $\mathbf{A}^{\prime}$ and dicyanoalkene $\mathbf{2}$. The main diastereomeric product trans-3 is formed via anti-D. The tin enolate $\mathbf{A}^{\prime}$ is considered to be the $Z$-form because of the interaction of the tin center with an $\mathrm{SH}$ group. ${ }^{1}$ In addition, a bulky $\mathrm{R}^{1}$ group would increase the $Z$-selectivity. A plausible transition state would be an eight-membered cyclic one (Heathcock's model). ${ }^{2}$ Considering the steric repulsion between $\mathrm{R}^{1}$ and $\mathrm{R}^{2}$, the transition structure (TS-I) leading to antiadduct $\mathbf{D}$ is favored over (TS-II). After the Michael addition, since the steric repulsion between $\mathrm{R}^{1}$ and $\mathrm{R}^{2}$ acts also in the cyclization stage, the route from anti-D to trans-3 is more advantageous than the route from syn-D to $c i s-\mathbf{4}$. However, trans-selectivity of dihydrothiophenes $\mathbf{3}$ is moderate even if $\mathrm{R}^{1}$ is ${ }^{t} \mathrm{Bu}$ group compared with the case using hydroxy ketones wherein complete trans-selectivity was attained. ${ }^{3}$ That is, the reaction of mercapto ketones $\mathbf{2}$ required severe conditions to cause the rearrangement from the thiolane 4. This rearrangement is not included in the reaction using hydroxy ketones. Therefore, the diastereoselectivity of $\mathbf{3}$ is lower in the case of mercapto ketones $\mathbf{1}$.

As a reference, the diastereoselectivity was not changed after heating for more serveral hours so no epimerization of the product $\mathbf{3 a a}$ would not occur.

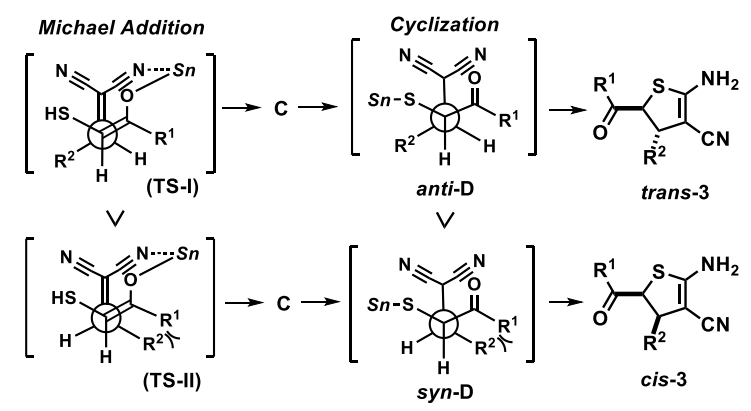

\section{Diastereoselectivity of the Thiolane 4}

At the stage of the ring formation (intermediate $\mathbf{B}$ ), stannyl keteneimine moietety is opposite to Ph group to avoid steric hindrance. The carbonyl group and the stanyl ketenimine is chelated by the tin center and Me group exists in less congested place. As a result, only the diastereomer $\mathbf{4}$ is formed.

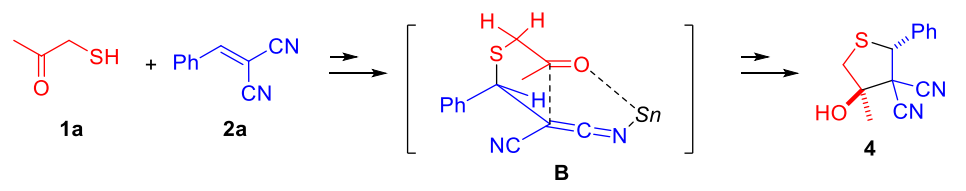

\footnotetext{
1 I. Shibata, K. Yasuda, Y. Tanaka, M. Yasuda and A. Baba, J. Org. Chem., 1998, 63, 1334.

For eight membered cyclic transition state: D. A. Oare and C. H. Heathcock, J. Org. Chem. 1990, 55, 157.

3 S. Tsunoi, Y. Takano, Y. Seo, I. Suzuki, I. Shibata, Org. Biomol. Chem. 2016, 14, 1707.
} 